\title{
SEARCH FOR THE STANDARD MODEL HIGGS BOSON IN
}

\section{PROTON-ANTIPROTON COLLISIONS AT A CENTER-OF-MASS ENERGY OF $1.96 \mathrm{TEV}$}

\author{
A Dissertation \\ Submitted to the Faculty \\ of \\ Purdue University \\ by \\ Viktor Veszpremi \\ In Partial Fulfillment of the \\ Requirements for the Degree \\ of \\ Doctor of Philosophy
}

August 2007

Purdue University

West Lafayette, Indiana 


\section{INTRODUCTION}

A scientifically accurate description of matter interpreted as a substance made up of corpuscular constituents was established during the course of the 19th century. In this description, atoms - the building blocks of the matter - form molecules. The properties of the molecules were described by chemistry or thermodynamics depending on what characteristics of the matter were investigated. In both theories, the molecules can dissociate to atoms when the kinetic energies of the atoms exceed the strength of the chemical bonds. The number of atoms is always preserved in a closed system. This is not true, however, when the matter takes up much higher energies at relativistic scales. New particles can be produced at the expense of the kinetic energy. The number of particles is no longer preserved. There are other conserved quantities, however, these quantities, the charge, baryon number, lepton number, are associated with particles that are considered elementary today. The properties and behavior of these elementary particles are the subject of Particle Physics or High Energy Physics.

\subsection{The Standard Model}

The Standard Model is an effective field theory that describes the properties of elementary particles (Appendix A), and provides understanding of how these particles interact with each other via the exchange of interaction mediating particles. Many experiments have been conducted in order to test the validity of this theory which has always proved to be true in the energy regime that can be explored with currently available technologies. A model in physics can be considered successful not only if it can describe already known phenomena but if it can also predict the existence of new ones. The Standard Model is considered a successful theory in both senses. 
In the theory of beta decays originally created by Fermi, it was assumed that particles decay instantaneously to three other particles in one point of space-time. This assumption worked at lower energies where the first order of the perturbation calculation was sufficient to describe the process. However, from a theoretical point of view, this model was expected to be wrong, because it violated unitarity at high energies and was not renormalizable. A charged massive vector boson was expected to mediate the weak interaction and to have a mass enough to explain the weakness of the interaction. For example, in the decay of a neutron, a negatively charged massive vector boson is emitted by the neutron as it becomes a proton, which then decays to an electron and a neutrino some distance away from the location where it was produced. The work of Glashow, Weinberg, and Salam in the 1960s predicted the required charged bosons together with a new one which was neutral.

They attempted to unify the electromagnetic and weak interactions via an $S U(2)$ non-Abelian gauge symmetry. The theory was renormalizable, but the bosons were predicted to be massless initially. The solution to the mass problem was provided by Higgs who suggested the application of spontaneous symmetry breaking in gauge theories by introducing a scalar field. The so called Higgs mechanism finally provided masses for the weak bosons. In the electroweak theory of the Standard Model, the masses of the $W^{ \pm}$and $Z^{0}$ were calculable from already known experimental results. Both weak bosons were observed subsequently in 1983 with the expected masses. The discovery of these particles assured physicists about the validity of the Standard Model. As a consequence of the Higgs mechanism, an additional heavy neutral particle should exists, called the Higgs boson. The Higgs boson is the last undiscovered particle of the Standard Model. If it is finally observed, its properties are going to determine the future of particle physics. 


\subsubsection{Forces in the Standard Model}

Interactions in the macroscopic world have a wide variety. However, they all can be viewed as a combination of four fundamental forces. These forces are - in increasing order of their strength - gravity, weak interaction, electromagnetic and strong interactions [1]. The Standard Model provides a concise treatment for the last three forces. It is still not known exactly what role gravity plays in particle physics.

The formalism of these interactions relies on the synergy of the theory of relativity and quantum physics. The former theory is used to describe the initial and final states of interactions in which the energy is conserved. Due to the energy-mass equivalence of special relativity, energy is allowed to turn into mass in the form of particles or vice versa within the constraints of conservation laws. Quantum physics allows for the interactions to actually take place. In quantum-mechanics, energy and momentum conservation can be violated for a short period of time, which is inverse proportional to the amount of extra energy appearing in the interaction. Particles interact with each other by exchanging bosons. In doing so, bosons violate the energy conservation for a short time.

Both bosons and fermions are particle fields which are described as functions of space-time. Bosons couple to the fermions via their charges listed in Table 1.1 and Table 1.2. These charges and the interactions they couple to are results of symmetries represented by the particle fields.

In the classical field theory of particle physics, the variation principle is applied on the space-time volume integral of the Lagrange density. The Lagrange density of a free, half-spin, massive particle is

$$
\mathcal{L}=\bar{\psi}\left(i \gamma_{\mu} \partial^{\mu}-m\right) \psi
$$

where $\gamma^{\mu}$ are the Dirac $\gamma$-matrices [2].

The variation of the action integral assuming that the field is fixed on the boundaries of the space-time volume in which the action integral is evaluated provides the equation of motion of the field, called the Dirac equation 
Table 1.1

Fundamental properties of fermions

\begin{tabular}{|c|c|c|c|}
\hline \multicolumn{4}{|c|}{ Leptons $(\operatorname{spin}=1 / 2)$} \\
\hline Particle & $\begin{array}{c}\text { Mass } \\
\left(M e V / c^{2}\right)\end{array}$ & $\begin{array}{c}\text { Electric } \\
\text { charge Q }\end{array}$ & $\begin{array}{c}\text { Hypercharge } \\
\text { Y/2 }\end{array}$ \\
\hline$\nu_{e}$ & $<3 \times 10^{-6}$ & 0 & $\left(\nu_{e}\right)_{L}=-1 / 2$ \\
\hline$e$ & 0.511 & \pm 1 & $\begin{array}{c}e_{L}=-1 / 2 \\
e_{R}=-1\end{array}$ \\
\hline$\nu_{\mu}$ & $<0.19$ & 0 & $\left(\nu_{\mu}\right)_{L}=-1 / 2$ \\
\hline$\mu$ & 106 & \pm 1 & $\begin{array}{c}\mu_{L}=-1 / 2 \\
\mu_{R}=-1\end{array}$ \\
\hline$\nu_{\tau}$ & $<18.2$ & 0 & $\left(\nu_{\tau}\right)_{L}=-1 / 2$ \\
\hline$\tau$ & $1.78 \times 10^{3}$ & \pm 1 & $\begin{array}{c}\tau_{L}=-1 / 2 \\
\tau_{R}=-1\end{array}$ \\
\hline \multicolumn{4}{|c|}{ Quarks $(\operatorname{spin}=1 / 2)$} \\
\hline up $(u)$ & $1.45-4.5$ & $+2 / 3$ & $\begin{array}{l}u_{L}=1 / 6 \\
u_{R}=2 / 3\end{array}$ \\
\hline down $(d)$ & $5-8.5$ & $-1 / 3$ & $\begin{array}{c}d_{L}=1 / 6 \\
d_{R}=-1 / 3\end{array}$ \\
\hline charm (c) & $(1.29 \pm 0.04) \times 10^{3}$ & $+2 / 3$ & $\begin{array}{l}c_{L}=1 / 6 \\
c_{R}=2 / 3\end{array}$ \\
\hline strange $(s)$ & $80-155$ & $-1 / 3$ & $\begin{array}{c}s_{L}=1 / 6 \\
s_{R}=-1 / 3\end{array}$ \\
\hline top $(t)$ & $(178 \pm 4.3) \times 10^{3}$ & $+2 / 3$ & $\begin{array}{l}t_{L}=1 / 6 \\
t_{R}=2 / 3\end{array}$ \\
\hline bottom (b) & $(4.21 \pm 0.03) \times 10^{3}$ & $-1 / 3$ & $\begin{array}{c}b_{L}=1 / 6 \\
b_{R}=-1 / 3\end{array}$ \\
\hline
\end{tabular}


Table 1.2

Fundamental properties of bosons

\begin{tabular}{|l|c|c|c|}
\hline \multicolumn{4}{|c|}{ Bosons (spin $=1)$} \\
\hline \hline Particle & $\begin{array}{c}\text { Mass } \\
\left(\mathrm{MeV} / \mathrm{c}^{2}\right)\end{array}$ & $\begin{array}{c}\text { Electric } \\
\text { charge Q }\end{array}$ & $\begin{array}{c}\text { Hypercharge } \\
\mathrm{Y}\end{array}$ \\
\hline$\gamma$ & 0 & 0 & 0 \\
\hline$W^{-}$ & $80.22 \times 10^{3}$ & -1 & -1 \\
\hline$W^{+}$ & $80.22 \times 10^{3}$ & +1 & +1 \\
\hline$Z^{0}$ & $91.19 \times 10^{3}$ & 0 & 0 \\
\hline gluons $(g)$ & 0 & 0 & 0 \\
\hline Higgs $(H)$ & $>114.4 \times 10^{3}$ & 0 & $1 / 2$ \\
\hline
\end{tabular}

$$
\left(i \gamma^{\mu} \partial_{\mu}-m\right) \psi=0
$$

A transformation that depends on the space-time coordinate is called a local transformation. If a physical system exhibits symmetry to such a transformation, it is called gauge invariant. Noether's theorem in classical field theory stated that for every symmetry transformation there exists a conserved quantity.

The idea was first applied in Quantum Electrodynamics, where the existence of the photons and their interactions with charged particles were derived from a $U(1)$ local gauge transformation. In order to make the Lagrangian symmetric in a local $U(1)$ transformation, one has to redefine the derivative of the particle field by introducing an auxiliary vector field (gauge field) in such a way that the expression containing the derivative also preserves the symmetry. The modified derivative is called the covariant derivative. It describes the interaction of the matter field with the newly introduced vector field, called the electromagnetic field. The coupling constant that determines the strength of the interaction between the gauge field and the particle is the electric charge. According to Noether's theorem, the electric charge is conserved. 
The other two forces of the Standard Model can also be introduced in a similar manner. In fact the three forces are based on an $S U(3) \times S U(2) \times U(1)$ symmetry. The couplings associated to these groups are expressed in terms of:

- Strong coupling constant $\alpha_{S}$ for the strong interactions

- Fermi constant $G_{F}$ for the weak interactions

- Electric charge $e$ for the electromagnetic interactions

\subsubsection{Quantum Electrodynamics}

Quantum Electrodynamics (QED) is the simplest example of how boson fields couple to the fermions. QED was developed in the 1940s and 1950s. It describes the electromagnetic interaction in a quantized relativistically invariant form. In particle physics, we assume that all interactions can be described by a gauge theory, therefore the theory of the weak and strong interactions are more complex generalized copies of QED [3].

The Lagrangian of a free massive fermion is given in Equation (1.1). This Lagrangian is not invariant under the $U(1)$ local transformation

$$
\psi \rightarrow e^{-i \alpha(x)} \psi
$$

where the parameter of the transformation $\alpha(x)$ is a function of the space-time coordinates. The problem emerges in the derivative of the transformed field, where the partial derivative of $\alpha(x)$ does not vanish. The solution follows by demanding the derivative to transform just as $\psi$ itself. This is called the covariant derivative:

$$
D_{\mu} \equiv \partial_{\mu}+i q A_{\mu}
$$

where $A_{\mu}(x)$ transforms as

$$
A_{\mu} \rightarrow A_{\mu}+\frac{1}{q} \partial_{\mu} \alpha
$$

The vector field $A_{\mu}$ is called the gauge field. In case of the $U(1)$ symmetry, this is the electromagnetic (EM) field. The quantum of the EM field is called the photon. 
Not only does the photon interact with charged particles, but it also has its own dynamics. The Lagrangian of the system of the fermion and the EM fields must be completed with the kinetic term of the photon. This is given by the gauge invariant field strength tensor

$$
F_{\mu \nu}=\partial_{\mu} A_{\nu}-\partial_{\nu} A_{\mu}
$$

in the form

$$
\mathcal{L}_{E M}=-\frac{1}{4} F_{\mu \nu} F^{\mu \nu}
$$

Important consequence of the gauge invariance of the Lagrangian is that quadratic terms for the vector field such as $m^{2} A_{\mu} A^{\mu}$ can not appear in the Lagrangian; therefore, the photon is massless.

\subsubsection{Electroweak Theory}

The theory of the weak interactions is constructed similar to QED, but the fundamental transformations are given by an $\mathrm{SU}(2)$ group. The dimension of $\mathrm{SU}(2)$ is 3 , therefore the weak interactions are mediated by three vector bosons following from the constructions of the covariant derivatives. There is a fundamental problem in the theory: the transformations do not allow for the vector bosons to have mass. This problem is solved in the framework of the Electroweak theory.

The Electroweak theory, which unifies QED and the weak interactions, is one of the greatest achievement of the Standard Model. The theory was formulated by Glashow, Salam, and Weinberg in the middle of the 1960s [4] [5].

Fermions (electrons and neutrinos in this example) are governed by the Lagrangian in (1.1). The Lagrangian can be written in terms of right-handed and left-handed components of the spinors given by

$$
\psi_{L}=\frac{1-\gamma_{5}}{2} \psi, \psi_{R}=\frac{1+\gamma_{5}}{2} \psi
$$

The left-handed components (an electron and a neutrino) form an isospin doublet $\psi_{L}=\left(e_{L}, \nu_{L}\right)$, whereas the right handed component (an electron) is an isospin singlet $\psi_{R}=\left(e_{R}\right)$. 
While requiring the Lagrangian to be invariant under the $S U(2)_{L}$ symmetry transformation (where the subscript $\mathrm{L}$ denotes the fact that the transformation changes only the left-handed spinors), the following covariant derivative is introduced:

$$
\partial_{\mu} \rightarrow D_{\mu}=\partial_{\mu}+i g \frac{\tau_{a}}{2} W_{\mu}^{a}+i \frac{g^{\prime}}{2} Y B_{\mu}
$$

The $W_{\mu}^{a}(\mathrm{a}=1,2,3)$ gauge fields correspond to the $S U(2)_{L}$ and $B_{\mu}$ to the $U(1)_{Y}$ transformations, $g$ and $g^{\prime}$ are the coupling constants, and $\tau^{a}$ are the Pauli spin matrices. Two of the $W_{\mu}^{a}$ fields are electrically charged, the third is neutral.

$Y$ is called the weak hypercharge which is equal to -1 for the (left-handed) fermion doublet and -2 for the (right-handed) fermion singlet. The hypercharge is related to the electric charge $Q$ and the weak isospin $\mathrm{T}$ as $Q=T_{3}+\frac{1}{2} Y$, which follows from the fact that $W_{\mu}^{3}$ interacts only with left-handed fermions $\left(T_{3}= \pm \frac{1}{2}\right)$ and $B_{\mu}$ couples to both left- and right-handed fermions $\left(T_{3}= \pm \frac{1}{2}\right.$ and $T_{3}=0$ respectively).

In their mass eigenstates, $W_{\mu}^{3}$ is mixed with $B_{\mu}$ via a rotation, which yields the physically observable EM vector boson $A_{\mu}$ and the neutral weak boson $Z_{\mu}$. The angle of the rotation is called the Weinberg angle $\Theta_{W}$. The physical fields are:

$$
W_{\mu}^{ \pm}=\frac{1}{\sqrt{2}}\left(W_{\mu}^{1} \mp i W_{\mu}^{2}\right)
$$

and

$$
\begin{gathered}
A_{\mu}=\cos \theta_{W} B_{\mu}+\sin \theta_{W} W_{\mu}^{3} \\
Z_{\mu}=-\sin \theta_{W} B_{\mu}+\cos \theta_{W} W_{\mu}^{3}
\end{gathered}
$$

where

$$
\frac{g^{\prime}}{g}=\tan \theta_{W}
$$

The field tensors are given as:

$$
\begin{gathered}
B_{\mu \nu}=\partial_{\mu} B_{\nu}-\partial_{\nu} B_{\mu} \\
W_{\mu \nu}=\partial_{\mu} W_{\nu}-\partial_{\nu} W_{\mu}+g W_{\mu} \times W_{\nu}
\end{gathered}
$$


The model predicted the existence of the $W^{ \pm}$and the $Z^{0}$ bosons before they were discovered. In this form of the theory, however, the gauge bosons are still massless, since a term in the Lagrangian such as (1.7) would not be invariant under gauge transformations. The solution is provided by the Higgs mechanism. It was proposed by P. Higgs in 1964 [6], but its validity still has not been proved experimentally. To demonstrate how the mechanism works, we describe the phenomenon of spontaneous symmetry breaking.

\subsubsection{Local and Global Spontaneous Symmetry Breaking}

Spontaneous symmetry breaking of a transformation group occurs when the Lagrangian of a scalar field exhibits a symmetry to the transformations, but the physical configuration of the field in its ground state no longer holds the same symmetry. According to Goldstone's theorem, in this case the violating components of the scalar field represent massless bosons. More precisely, consider a vector of $\mathrm{N}$ scalar fields and a corresponding group of continuous global transformations to which the Lagrangian is invariant. If we choose the ground state of the system so that an $\mathrm{n}$ dimensional sub-group of the transformation is no longer a symmetry of the physical ground state then $\mathrm{n}$ of the $\mathrm{N}$ scalar fields (expressed in the eigen-states of the mass-tensor) are going to be massless.

We only illustrate this statement here. Let us consider a complex scalar field $\phi=\left(\phi_{1}, \phi_{2}\right)$ with the following Lagrangian:

$$
L=\partial_{\mu} \phi^{*} \partial^{\mu} \phi-V\left(\phi^{*} \phi\right)
$$

where the potential is

$$
V\left(\phi^{*} \phi\right)=\mu^{2}\left(\phi^{*} \phi\right)+\lambda\left(\phi^{*} \phi\right)^{2}
$$

and $\lambda$ is positive so that the potential has a minimum. This Lagrangian is invariant under the global phase transformation

$$
\phi \rightarrow e^{-i \Theta} \phi
$$


Let's express the field with the real scalar components

$$
\phi=\frac{\phi_{1}+i \phi_{2}}{\sqrt{2}}
$$

Plugging this formula into the Lagrangian, we get

$$
L=\frac{1}{2}\left(\partial_{\mu} \phi_{1} \partial^{\mu} \phi_{1}+\partial_{\mu} \phi_{2} \partial^{\mu} \phi_{2}\right)-V\left(\phi_{1}, \phi_{2}\right)
$$

In the minimum energy state of the field (ground state), the derivative of equation (1.17) vanishes. If $\mu^{2}>0$, the field must also vanish, therefore $\phi_{1}=\phi_{2}=0$. In this case, the ground state preserves the symmetry of the transformation (1.18), and the Lagrangian of the field for small oscillations can be expressed as the sum of two Lagrangians of independent real scalar fields of mass $\mu(1.20)$.

If $\mu^{2}<0$, the field has a non-zero ground state. If we wrote the complex field in the form $\phi \propto \phi_{1} \cdot e^{i \phi_{2}}$, it is obvious, that the potential would be symmetric to the change of $\phi_{2}$, in this case, the complex phase. The choice of the complex phase is completely free. After finding the minimum of the potential, we obtain that $|\phi|= \pm \sqrt{\frac{-\mu^{2}}{2 \lambda}}$. We choose a ground state

$$
\begin{aligned}
& \phi_{1}=v, \\
& \phi_{2}=0
\end{aligned}
$$

where

$$
v^{2}=\frac{-\mu^{2}}{\lambda}
$$

We consider the field as perturbation about this configuration

$$
\begin{gathered}
\phi_{1}^{\prime}=\phi_{1}-v, \\
\phi_{2}^{\prime}=\phi_{2}
\end{gathered}
$$

The new Lagrangian following from (1.20) will contain a field $\phi_{1}^{\prime}$ with mass $\mu=$ $v \sqrt{2 \lambda}$, the Goldstone boson $\phi_{2}^{\prime}$ with zero mass, plus the interaction terms:

$$
L=\frac{1}{2} \partial_{\mu} \phi_{1}^{\prime} \partial^{\mu} \phi_{1}^{\prime}+\lambda v^{2} \phi_{1}^{\prime 2}+\frac{1}{2} \partial_{\mu} \phi_{2}^{\prime} \partial^{\mu} \phi_{2}^{\prime}+\text { interact }
$$


We have not discovered any massless scalar particles that Goldstone's theorem predicts. This contradiction, however, is saved by the Higgs mechanism. According to this model, the massless Goldstone bosons do not appear as individual particles, but are "absorbed" by the gauge bosons of the Electroweak theory. Due to this phenomenon, the gauge bosons gain mass in such a way that the electroweak Lagrangian stays invariant to local gauge transformations. To make this work, the Lagrangian in equation (1.16) needs to be invariant to the local transformation of $\phi$ :

$$
\phi \rightarrow e^{-i \alpha(x)} \phi
$$

The transformation equations of the field and its derivative in the Lagrangian are given in (1.25), (1.4), and (1.5). Let us express the complex field in the following form:

$$
\phi=e^{i \frac{\phi_{2}^{\prime}}{v}} \cdot \frac{\left(\phi_{1}^{\prime}+v\right)}{\sqrt{2}} \approx \phi^{\prime}+\frac{v}{\sqrt{2}}
$$

The Lagrangian becomes:

$L=\frac{1}{2} \partial_{\mu} \phi_{1}^{\prime} \partial^{\mu} \phi_{1}^{\prime}-\lambda v^{2} \phi_{1}^{\prime 2}+\frac{1}{2} \partial_{\mu} \phi_{2}^{\prime} \partial^{\mu} \phi_{2}^{\prime}+$ interact. $-\frac{1}{4} F_{\mu \nu} F^{\mu \nu}+\frac{q^{2} v^{2}}{2} A_{\mu} A^{\mu}+q v A_{\mu} \partial^{\mu} \phi_{2}^{\prime}$

where $M_{A}=q v$, the mass of the vector boson $A_{\mu}$, depends on the vacuum expectation value $v$ of the field $\phi$. This equation still contains a massless scalar field $\phi_{2}$ and an interaction term with $A_{\mu}$. The choice of $\phi_{2}$ at this point is arbitrary, so is the phase of $A_{\mu}$ according to (1.5). The Goldstone boson can be eliminated from the Lagrangian by choosing the gauge parameter (unitary gauge) such as:

$$
\alpha(x)=-\frac{1}{v} \phi_{2}^{\prime}(x)
$$

The form of $\phi$ in (1.26) can now be further simplified to

$$
\phi=\frac{1}{\sqrt{2}}\left(\phi_{1}^{\prime}+v\right)
$$

and the final Lagrangian

$$
L=\frac{1}{2} \partial_{\mu} \phi_{1}^{\prime} \partial^{\mu} \phi_{1}^{\prime}-\lambda v^{2} \phi_{1}^{\prime 2}-\frac{1}{4} F_{\mu \nu} F^{\mu \nu}+\frac{q^{2} v^{2}}{2} A_{\mu} A^{\mu}+\frac{1}{2} q^{2}\left(\phi_{1}^{\prime}+2 v\right) \phi_{1}^{\prime} A_{\mu} A^{\mu}-\frac{\lambda}{4} \phi_{1}^{\prime 3}\left(\phi_{1}^{\prime}+4 v\right) .
$$


It describes a massive scalar field $\phi_{1}^{\prime}$, called the Higgs boson, and a massive vector boson $A_{\mu}$.

\subsubsection{Higgs Mechanism in the Weinberg-Salam Model}

The Higgs field of the Standard Model is expressed as a complex doublet $\Phi=$ $\left(\Phi^{+}, \Phi^{0}\right)$. The ground state of the Higgs field is chosen so that it breaks the $S U(2)$ and $U(1)_{Y}$ symmetries but not the $U(1)_{E M}$, therefore it must have $Y=1$ hypercharge and in the ground state

$$
\Phi_{0}=\frac{1}{\sqrt{2}}\left(\begin{array}{l}
0 \\
v
\end{array}\right)
$$

where $v$ was chosen as in (1.22). The Higgs field is expressed as a perturbation about this vacuum-state. As in the example of the previous section, the Higgs field components with zero ground state are eliminated from the equations by choosing a unitary gauge. The remaining field is:

$$
\Phi=\frac{1}{\sqrt{2}}\left(\begin{array}{c}
0 \\
v+h
\end{array}\right)
$$

The gauge bosons gain mass via interacting with the Higgs field. Upon evaluation of the kinetic term in (1.16) using the covariant derivative of the Electroweak interaction (1.9):

$$
\left(D_{\mu} \Phi\right)^{\dagger}\left(D_{\mu} \Phi\right)=\frac{1}{2}\left(\partial_{\mu} h\right)^{2}+\frac{g^{2} v^{2}}{4}\left[\frac{\left(W_{\mu}^{1}\right)^{2}+\left(W_{\mu}^{2}\right)^{2}}{2}\right]+\frac{v^{2}}{8}\left(g W_{\mu}^{3}-g^{\prime} B_{\mu}\right)^{2}+\text { interact. }
$$

The first term in the result describes a massive scalar field $h$, the Higgs particle, which is the subject of this analysis. Its mass $v \sqrt{2 \lambda}$ follows from the potential term $(1.17)$ which was omitted in (1.33). The second term gives mass to the physical $W^{ \pm}$bosons when using (1.10), the third one gives mass to the $Z^{0}$ boson from (1.12) and (1.13). Since there is no term with a quadratic form of $A_{\mu}$, the photon remains massless (by construction). The mass of $W^{ \pm}$and $Z^{0}$ are:

$$
m_{W}=\frac{g v}{2}
$$


and

$$
m_{Z}=\frac{g v}{2 \cos \theta_{W}}=\frac{m_{W}}{\cos \theta_{W}}
$$

In summary, the $U(1)_{\text {em }}$ symmetry conservation results in a zero mass photon and a massive scalar field (one of the four components of $\Phi$ ) called the Higgs boson. In the $S U(2)$ symmetry breaking, three Goldstone bosons are created (the other three real components of $\Phi)$. Choosing the proper gauge parameters for the $S U(2)$ the three massless bosons are absorbed by the three weak bosons, giving mass to $W^{ \pm}$and $Z^{0}$.

The mass of the $W^{ \pm}$was expected from earlier theories, however, the $Z^{0}$ mass and its relation to the $W^{ \pm}$-mass is truly a prediction. Both particles were found in 1983 by the UA1 Collaboration [7] and [8] with their right mass. The theory, however, does not yield direct information on the Higgs mass. The vacuum expectation value $v=246 \mathrm{GeV}$ is calculated from the equations above, but $\lambda$ remains a free parameter. One of the greatest challenges in experimental High Energy Physics is to find the Higgs boson and explore its interactions.

\section{Mass of the Fermions}

The fermion masses can also originate from interactions with the Higgs field by Yukawa couplings. The Lagrangian with the spinor fields from (1.8) and the Higgs from (1.32)

$$
\mathcal{L}=-G_{e}\left[\bar{\psi}_{L} \Phi \psi_{R}+\bar{\psi}_{R} \bar{\Phi} \psi_{L}\right]
$$

The coupling constants $G_{e}$ are arbitrary, therefore they do not provide any prediction. With the choice

$$
m_{f}=\frac{G_{e} v}{\sqrt{2}}
$$

the mass term in the Lagrangian takes the form

$$
-m_{f} \bar{f} f\left(1+\frac{h}{v}\right)
$$




\subsubsection{Quantum Chromodynamics}

Quantum Chromodynamics (QCD) describes the interaction between quarks. In the baryon model, three quarks can form a bound state. However, two fermions cannot occupy the same quantum-state according to Pauli's principle. The contradiction is resolved by assuming a new charge based on which the three quarks in the baryon can be distinguished. This charge is called color. QCD is an $S U(3)$ symmetry and it can be derived in analogy to QED. The existence of three color states and eight mediator bosons follows from the formalism.

This time the Lagrangian of the system is expressed in terms of the quark field $q=\left(q_{1}, q_{2}, q_{3}\right)$. Since $\mathrm{SU}(3)$ operates on three-dimensional vectors, we need three components to describe three color states of a quark of a given flavor.

$$
\mathcal{L}=\bar{q}_{j}\left(i \gamma^{\mu} \partial_{\mu}-m\right) q_{j}
$$

We require the Lagrangian to be invariant under the local transformation

$$
q(x) \rightarrow e^{i \alpha_{a}(x) T_{a}} q(x)
$$

where $T_{a}$ are eight traceless matrices that serve as the basis of a $3 \times 3$ unitary transformation. Since these matrices do not all commute with each other, their group is non-Abelian. Again, the Lagrangian is not invariant under this transformation, unless we introduce the covariant derivative.

$$
D_{\mu} \equiv \partial_{\mu}+i g T_{a} G_{\mu}^{a}
$$

In this case, eight gauge fields are needed to complete the symmetry which transform as

$$
G_{\mu}^{a} \rightarrow G_{\mu}^{a}-\frac{1}{g} \partial_{\mu} \alpha^{a}-f_{b c}^{a} \alpha^{b} G_{\mu}^{c}
$$

where $f^{a b c}$ are the structure constants of the group determining the commutation relations between the elements of the $\mathrm{SU}(3)$ transformation group. The eight gauge fields imply the existence of eight gluons. We can also define the field strength tensor with the following equation

$$
F_{\mu \nu}^{a} \equiv \partial_{\mu} G_{\nu}^{a}-\partial_{\nu} G_{\mu}^{a}+g f^{a b c} G_{\mu}^{b} G_{\nu}^{c}
$$


The last term that did not appear in the formula of the EM field tensor is due to the non-zero commutation between the field components. It is still possible, however, to construct an invariant square out of $F^{a}$ that is responsible for the dynamics of the field similarly to the EM case

$$
\mathcal{L}=-\frac{1}{4} F_{\mu \nu}^{a} F_{a}^{\mu \nu}
$$

The following statements summarize the results of this brief consideration of the SU(3) symmetry

- Due to the dimension of the transformation group, there are three charges associated with each component of the quark field. These are conventionally named after colors: red $(R)$, green $(G)$, and blue $(B)$. Naturally, anti-quarks have anti-colors: $\bar{R}, \bar{G}$, and $\bar{B}$. The motivation behind this terminology is that all bound states that have been observed in experiments so far are white when using color addition. The mesons are bound states of color and anti-color quark pairs, whereas the baryons contain three quarks in one of each color.

- There are eight gluons corresponding to the eight linearly independent transformations of $\mathrm{SU}(3)$. The quarks interact with each other by gluon exchanges.

- The part of the Lagrangian responsible for the dynamics of the gluon field, similarly to QED, would not be gauge invariant if the gluons were massive particles.

- Due to the non-Abelian nature of the transformation group, there is an additional quadratic term of the vector field in the energy tensor that prescribes self-interaction to the gluons; therefore, gluons can also interact with each other. This means that the gluons carry color charges themselves. In fact, the eight physical gluons are the eight possible anti-symmetric combinations of color and anti-color. 
QCD interactions behave very differently from QED. An electrically charged particle placed in vacuum experiences a so called screening effect due to polarization of the virtual electron-positron pairs being present in the vacuum. The QED coupling constant is a logarithmically increasing function of the momentum transfer between two charged particles. In QCD, gluon-gluon interactions produce anti-screening; the coupling constant is a decreasing function of the momentum-transfer, but it increases with distance. The former is called asymptotic freedom, the latter is color confinement. Consequently, at very high energies, the quarks can be treated as free particles, but they can not be separated. Therefore there is no stable single quark state, and there are no free gluons.

The mass of quarks can also be modeled by (1.38). The quark mass eigenstates are not the same as the weak eigenstates. The transformations from one eigenstate to the other is described by the Cabibbo-Kobayashi-Maskawa (CKM) matrix $V_{C K M}$ (1.45) which describes a rotation of the weak isospin eigenstates through the Cabibbo angle [9]. The transformation provides the weak interaction with the ability to change the flavor of quarks.

$$
\left(\begin{array}{c}
d^{\prime} \\
s^{\prime} \\
b^{\prime}
\end{array}\right)=\left(\begin{array}{ccc}
V_{u d}=0.974 & V_{u s}=0.23 & V_{u b}=0.004 \\
V_{c d} \approx 0.24 & V_{c s} \approx 0.97 & V_{c b}=0.04 \\
V_{t d}<0.014 & V_{t s}<0.043 & V_{t b} \approx 1
\end{array}\right)\left(\begin{array}{c}
d \\
s \\
b
\end{array}\right)
$$

The matrix implies that there are favored and suppressed transitions (making the $t \bar{t}$ process one of the largest backgrounds in this analysis).

Due to the strength of the QCD coupling, events observed at the Tevatron are mainly produced in QCD interactions. We need to consider those events where a heavy flavor quark (primarily b-quark) is created. These processes are: flavor creation, flavor excitation, and initial as well as final state radiation. In the energy range of this search, most events are initiated by the incident quarks, and the b-quarks are produced either by direct production or by gluon splitting. 

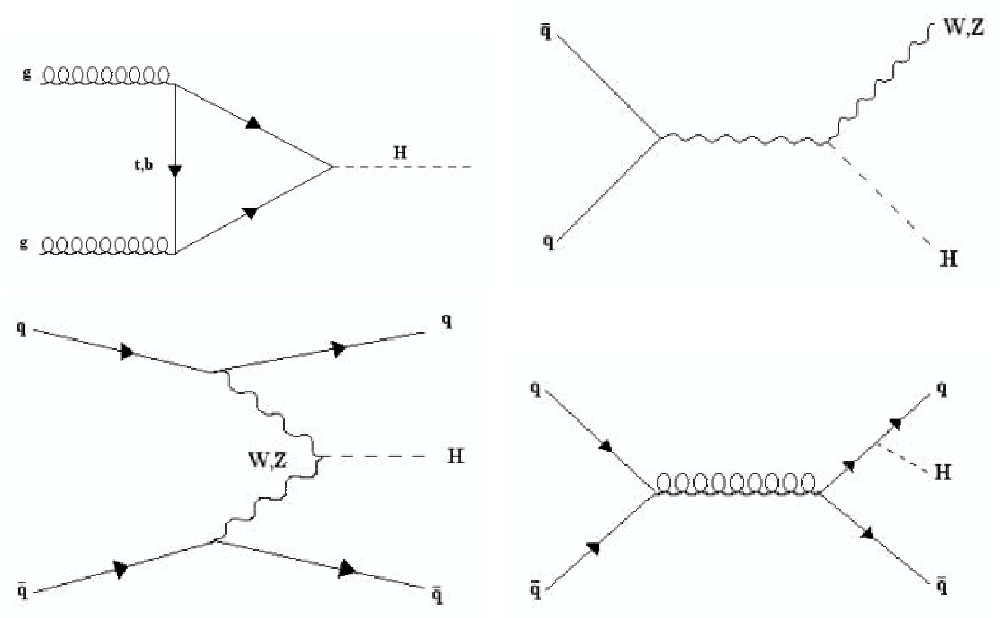

Fig. 1.1. Higgs production processes at hadron colliders: gluon fusion, "Higgs-strahlung", vector boson fusion, and $q \bar{q}$ associated productions $(q=t, b)$

\subsection{Higgs Boson at the Tevatron}

The Higgs coupling to fermions and bosons was introduced in Section 1.1.5. The tree-level coupling strength is a linear function of the fermion and boson masses. The dominant mechanisms therefore involve the heaviest particles: the $W^{ \pm}, Z^{0}$ bosons and the third generation quarks and leptons. At higher order, involving one loop processes, the Higgs also couples to gluons and photons. Diagrams of the most important processes are shown in Figure 1.1.

\subsubsection{Higgs Production}

Figure 1.2 shows the cross-section of the various production modes evaluated at the Tevatron center of mass energy [10]. The most important production modes are:

- $g g \rightarrow h_{S M}$, gluon fusion is the process with by far the largest cross-section at the Tevatron around $1.0 \mathrm{pb}-0.2 \mathrm{pb}$ for $115 \mathrm{GeV} \leq m_{h_{S M}} \leq 200 \mathrm{GeV}$. Gluon fusion happens primarily through top-loops, since the top is the heaviest quark. The two-loop QCD corrections contribute to the total cross-section by $60-100 \%$ [11]. 


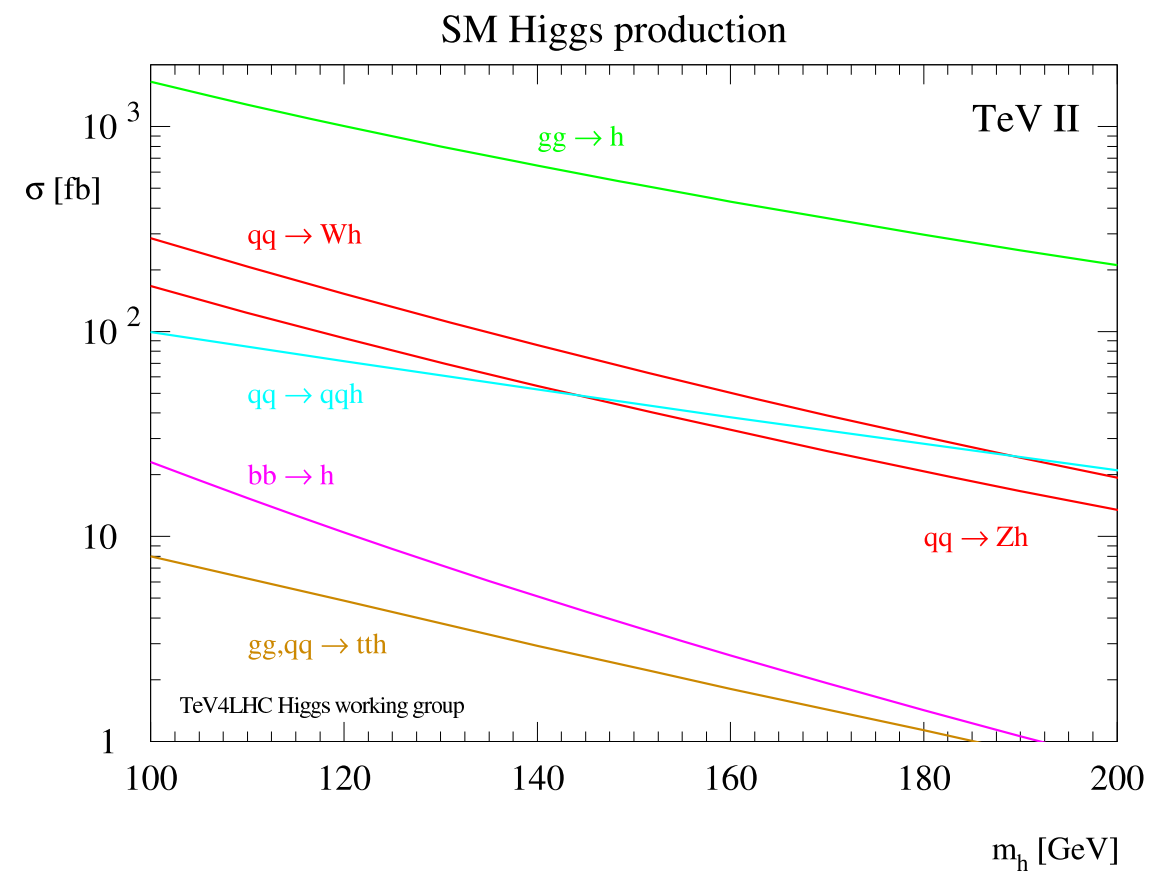

Fig. 1.2. Production cross-section of the Standard Model Higgs boson in various processes at the Tevatron

- $q \bar{q} \rightarrow V^{*} \rightarrow V h_{S M}$ where $V=W^{ \pm}, Z^{0}$, "Higgs-strahlung" or vector boson associated production is the process with the second highest cross-section in the mass region below $140 \mathrm{GeV}$. Its cross-section is around $0.1 \mathrm{pb}-0.3 \mathrm{pb}$ for Higgs mass at 110-120 GeV. The QCD corrections to this channel amount to $30 \%[12]$.

- $q \bar{q} \rightarrow q \bar{q} h_{S M}$, the vector boson fusion has the second largest cross-section in the high mass region. The quark and anti-quark both radiate a vector boson which then annihilate into a Higgs boson. Figure 1.2 shows the cross-section of all the contributing processes. The total cross-section is about $0.3 \mathrm{pb}$ for Higgs mass around $140 \mathrm{GeV}$. The distinct signature of this process is provided by the two forward jets. The vector boson associated production can also produce similar final states. 


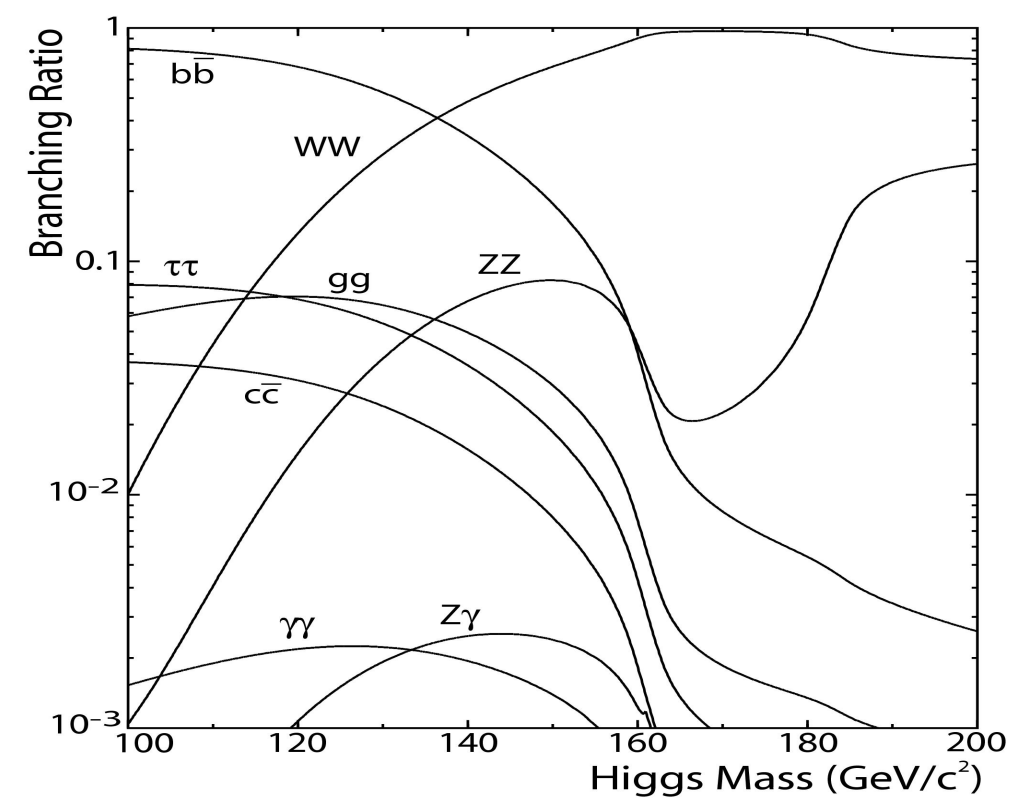

Fig. 1.3. Branching fraction of the Standard Model Higgs decay processes as a function of the Higgs mass

- $q \bar{q}, g g \rightarrow h_{S M} Q \bar{Q}$ where $Q=t, b$. It is worth mentioning the associated production of a Higgs boson and a heavy quark pair. Until recently, the $t \bar{t}$ associated production was considered as one of the production modes most viable for analysis at the LHC. At the Tevatron, the total integrated luminosity is not enough to compensate for the very small cross-section $0.05 \mathrm{pb}$ at $110-120 \mathrm{GeV}$. In some extensions of the Standard Model (MSSM with large $\tan \beta$ ), the $b \bar{b}$ associated production is enhanced. Further complication with analyses in the four-b final state (if $h_{S M} \rightarrow b \bar{b}$ ) is that it is well hidden in the heavy flavor multi-jet SM background which is difficult to estimate.

\subsubsection{Decays of the Higgs}

The branching fractions of the Higgs boson are shown in Figure 1.3. The daughters at leading order are also heavy particles due to the mass-couplings. Therefore, they further decay to stable particles. In some cases, the decay products are not distinct 
from the SM background, and searches need to rely on associated Higgs production modes.

Higgs bosons with mass $m_{h_{S M}}<130 \mathrm{GeV}$ decay predominantly to b-quark pairs [11]. The b-quarks appear as jets of particles in the detector. The Standard Model multi-jet background is mostly produced in QCD processes. This background is greatly reduced by taking advantage of the long lifetime of b-hadrons which is resolvable in the tracking system. The heavy flavor production in QCD processes nevertheless remains orders of magnitude higher than the Higgs cross-section. In the low mass region, the most viable searches are performed in the associated production channels. In the vector boson associated channels, it is possible to significantly reduce the QCD background by triggering on the decay products of the $W^{ \pm} / Z^{0}$ bosons.

When $m_{h_{S M}}>130 \mathrm{GeV}$, the $h_{S M} \rightarrow W^{+} W^{-}$decay becomes dominant. The $W^{ \pm}$ bosons further decay to well-detectable leptons in a unique topology determined by angular momentum conservation. In this mass region, therefore, most of the Higgs bosons are produced via gluon fusion. At very high masses, the $Z Z$ production is also significant, which can lead to very unique four-lepton final states.

\subsubsection{Higgs Searches}

The Electroweak theory of the Standard Model as described in the theoretical introduction assumes that weakly coupled scalar fields are responsible for the electroweak symmetry breaking [13]. There are other classes of electroweak symmetry breaking mechanisms:

- strong symmetry breaking, that introduces new strong interactions near the $\mathrm{TeV}$ scale

- the Little Higgs models where the lightest scalar resembles the weakly-coupled Higgs boson,

- large extra dimension theories in which new spatial dimensions are introduced, 


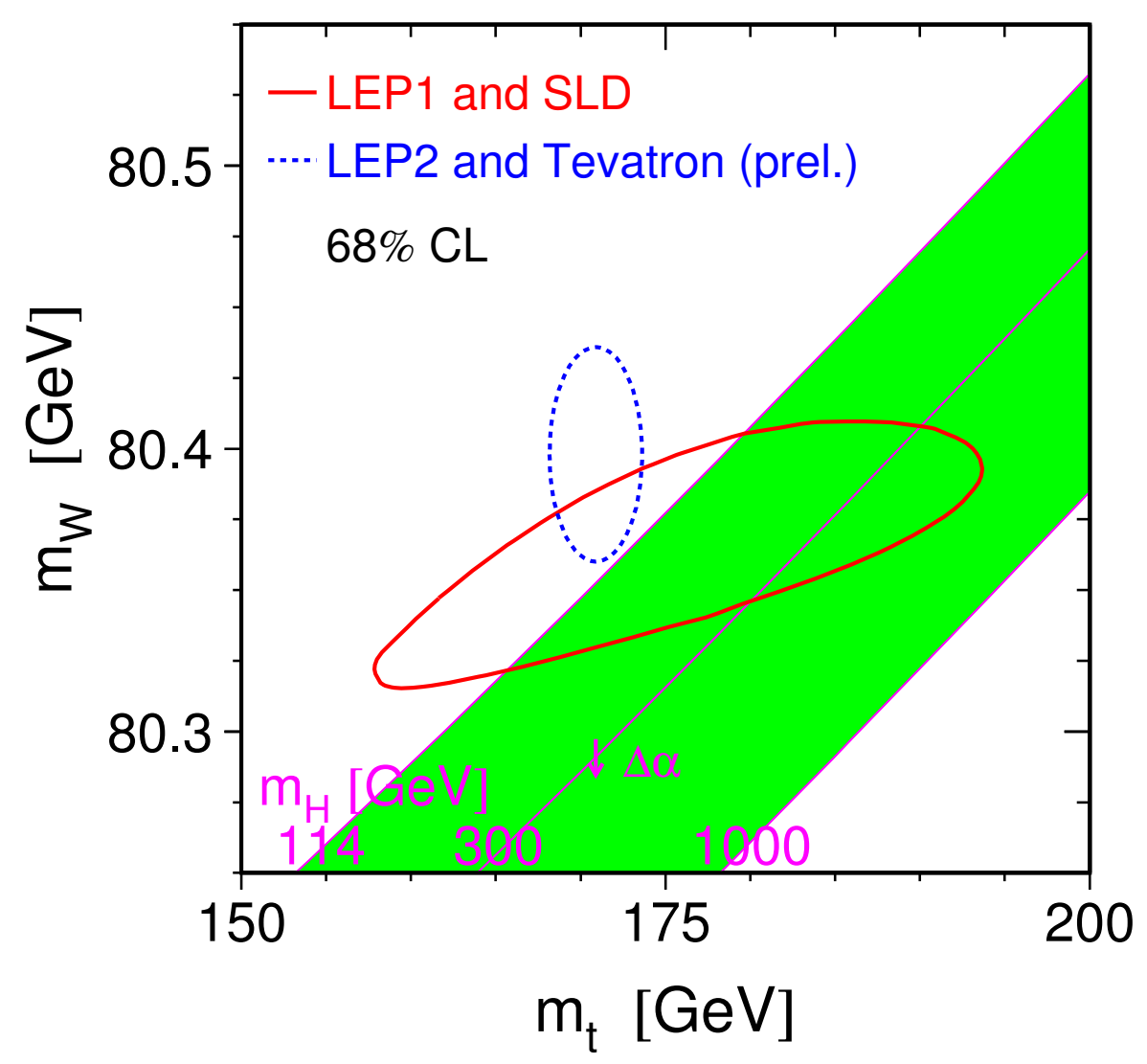

Fig. 1.4. The $W^{ \pm}$mass versus the indirectly and directly measured top masses. The diagonal lines show the corresponding Higgs mass beyond the current limit of $114 \mathrm{GeV}$. The small overlap between the two regions suggests a light Higgs [14].

- Higgsless models based on dynamical symmetry breaking

Although, there is no direct evidence for the weakly-coupled Higgs theory, the precision electroweak data collected at the LEP, SLC, and the Tevatron strongly support it. New physics that could enter through vacuum polarization corrections to the $W^{ \pm}, Z^{0}$ bosons has become very much constrained. 
The Higgs boson mass is not theoretically predicted, but it is possible to give an approximate estimate for its mass using theoretical considerations and precision measurements. The Higgs boson contributes to the propagator of the $W^{ \pm}, Z^{0}$ bosons through loop-effects. The corrections are logarithmic functions of the Higgs mass leading to limited conclusions. The LEP Electroweak Working Group has been performing global fits to the electroweak data (Figure 1.4). The Higgs mass is currently constrained to $m_{h_{S M}}=76_{-24}^{+33} \mathrm{GeV}(68 \% \mathrm{CL})$, and $m_{h_{S M}}<144 \mathrm{GeV}$ with $95 \% \mathrm{CL}$. Figure 1.5 also shows that the best fit corresponds to a Higgs below the exclusion region [14].

Direct searches performed in the LEP experiments have constrained the Higgs mass to be larger than $114.4 \mathrm{GeV}$ at $95 \%$ C.L [15]. As the Tevatron is now taking data at high instantaneous luminosities, it might be possible to set higher limits on the Higgs mass or even to find evidence for its existence.

The following searches are performed at the Tevatron currently:

1. $m_{h_{S M}}<150 \mathrm{GeV}$ with associated production and $h_{S M} \rightarrow b \bar{b}$ decays

- $\ell^{ \pm} \nu b \bar{b}$ channel: the Higgs is produced in association with a $W^{ \pm}$which has a relatively large cross-section. Missing energy, lepton-identification, and b-jet selection are used

- $\ell^{+} \ell^{-} b \bar{b}$ channel with $Z^{0}$-associated production. It provides the cleanest signal among the low mass Higgs searches due to the two leptons in the Z-mass resonance and b-tagging.

- $\nu \nu b \bar{b}$ channel: it is sensitive to both $W^{ \pm}$and $Z^{0}$ associated processes thus having a large signal cross-section, but the missing energy variables possess weak selection power.

- $q \bar{q} b \bar{b}$ channel: it has by far the largest cross-section among the Higgs searches. Roughly $70 \%$ of the $W^{ \pm}$and $Z^{0}$ associated processes provide this signature, moreover, this final state also arises in vector boson fusion processes. Nevertheless, this is probably the most difficult search due to 


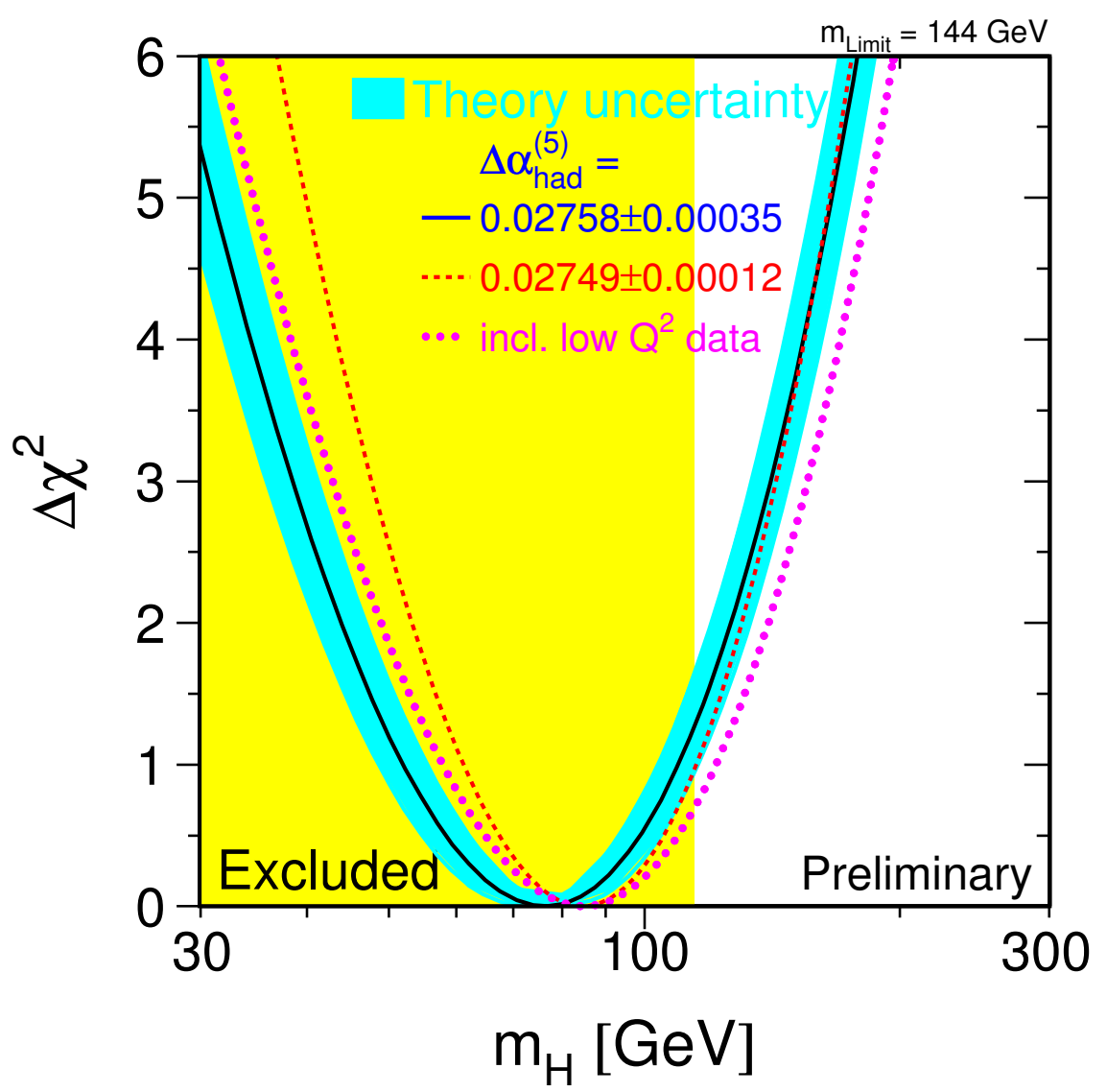

Fig. 1.5. $\chi^{2}$ of the global fit to the Standard Model as a function of the Higgs mass in three different scenarios for the hadronic correction to $\alpha_{e m}[14]$.

the large cross-section and difficult modelling of the SM multi-jet background with a heavy flavor jet pair.

- $W^{+} W^{-} b \bar{b} b \bar{b}$ channel is based on $t \bar{t}$ associated Higgs production with very small cross-section.

2. $m_{h_{S M}}>130 \mathrm{GeV}$ with $h_{S M} \rightarrow W^{+} W^{-}$decays 
- $\ell^{+} \ell^{-} \nu \nu$ channel is by far the most sensitive among the searches with no jets and two leptons in a back-to-back configuration.

- $W^{ \pm} W^{+} W^{-}$channel with $W^{ \pm}$-associated production. It is based on likesign dilepton identification with very small background but also small effective cross-section.

We are analyzing Z- and W-Higgs associate productions (Figure 1.1) where the $\mathrm{Z}$ decays to two neutrinos and the $\mathrm{W}$ decays to unidentified leptons. Because the neutrinos will not be detected in the calorimeter, they lead to imbalanced transverse energy in the detector. We do not expect isolated leptons in these events. Using a set of loose lepton selection requirements we can veto events with isolated leptons and reduce the $\mathrm{W} / \mathrm{Z}+$ jets background. We are performing a blind analysis in which all Standard Model background processes are simulated such as QCD heavy flavor multi-jet production, top production, and electro-weak processes. 


\section{EXPERIMENTAL APPARATUS}

Searches for the Standard Model Higgs boson have been pursued in high energy beam colliders. In these machines, collimated bunches of elementary or composite particles are accelerated in directions opposite to each other. In certain points, the bunches are crossed, and interactions occur between particles from each beam. The purpose of the experiment is to study inelastic collisions in which new particles are created. The type of the interactions can be reconstructed and quantified by detecting the outgoing particles. The most important parameters associated with colliders are the beam energy, the number of particles in the bunches, and the frequency at which the bunches are crossed. These parameters determine how massive particles can be produced and at what frequency.

\subsection{The Tevatron}

The Tevatron is a proton-antiproton collider located at Fermi National Laboratory in Batavia, Illinois [16]. It is the world's highest energy accelerator at the present. The $6.3 \mathrm{~km}$ (4 miles) circumference ring stores protons and antiprotons in 36 bunches where the length of one bunch is $19 \mathrm{~ns}$ and the spacing between the bunches is 396 ns [17]. The bunches are equally divided into three $4.4 \mu \mathrm{s}$ bunch trains separated by $2.6 \mu$ s abort gaps. The bunches are crossed in two points, where the protons and antiprotons collide with a center of mass energy of $\sqrt{s}=1.96 \mathrm{TeV}$.

Both the protons and antiprotons go through a series of smaller accelerators before being injected into the main ring. (Figure 2.1) The protons are obtained from Hydrogen gas. $\mathrm{H}^{-}$ions are created by ionizing the gas. The ions are accelerated to $750 \mathrm{keV}$ kinetic energy in the Cockcroft-Walton pre-accelerator and passed to the Linac (linear accelerator). The $150 \mathrm{~m}$ long Linac consists of 9 Radio Frequency (RF) 


\section{FERMILAB'S ACCELERATOR CHAIN}

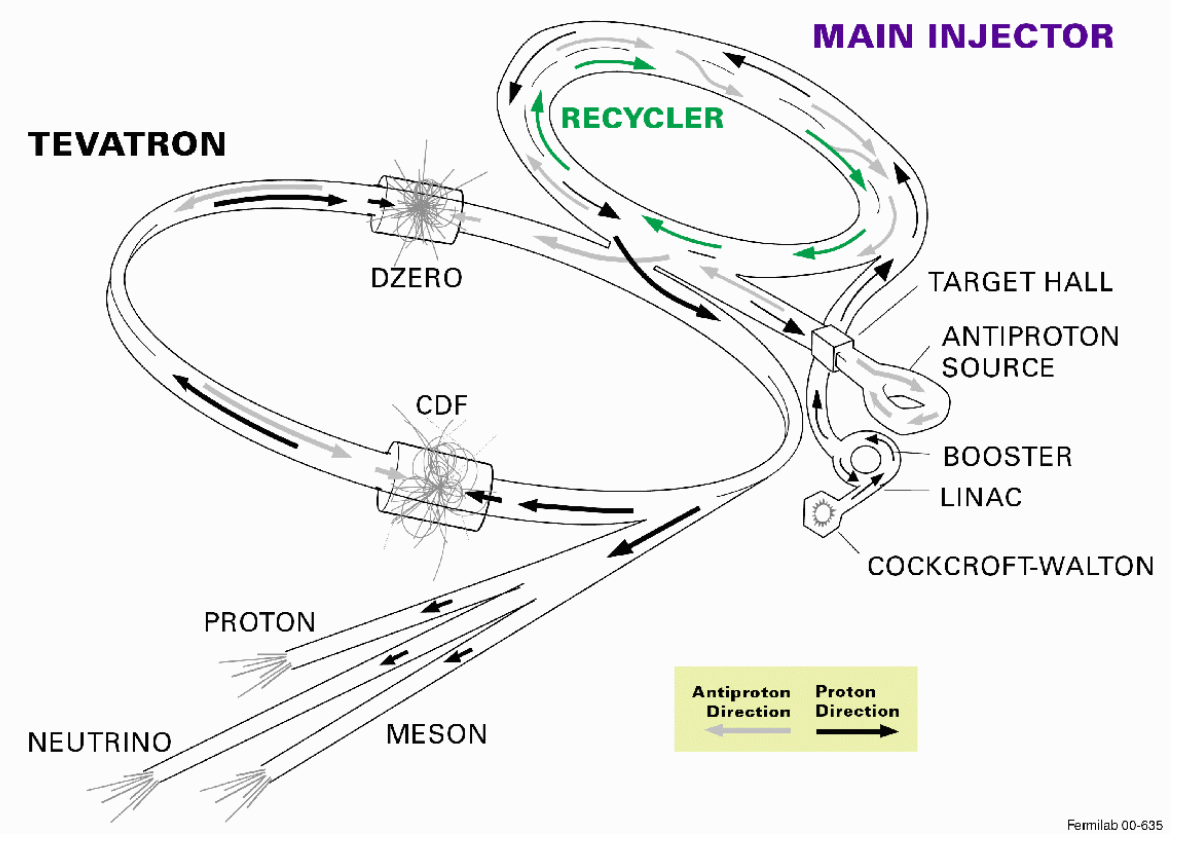

Fig. 2.1. Schematic overview of the acceleration process at the Tevatron

cavities in which EM-waves run through the Linac. The $\mathrm{H}^{-}$ions ride these waves such that ions which are ahead of the nearest minima of the waves are accelerated less then those which are behind. At the end of the accelerator, the ions are moving in bunches with $400 \mathrm{MeV}$ kinetic energy. The hydrogen ions then are passed through a carbon foil which strips their electrons off. The next step of the acceleration happens in the Booster, a circular synchrotron of $74.5 \mathrm{~m}$ diameter, where the protons gain $8 \mathrm{GeV}$ energy. The Booster circulates 12 bunches of protons at a time. The 12 bunches are extracted to the $3 \mathrm{~km}$ long Main Injector, where six of them are ready to be injected into the Main ring at $150 \mathrm{GeV}$ energy. The bunches are also concentrated into $19 \mathrm{~ns}$ long high density buckets.

The Main Injector is also used to produce antiprotons. The other six bunches of protons are directed to a nickel target when their energy reaches $120 \mathrm{GeV}$. In the collision, antiprotons are produced among many other particles. $8 \mathrm{GeV}$ antiprotons 


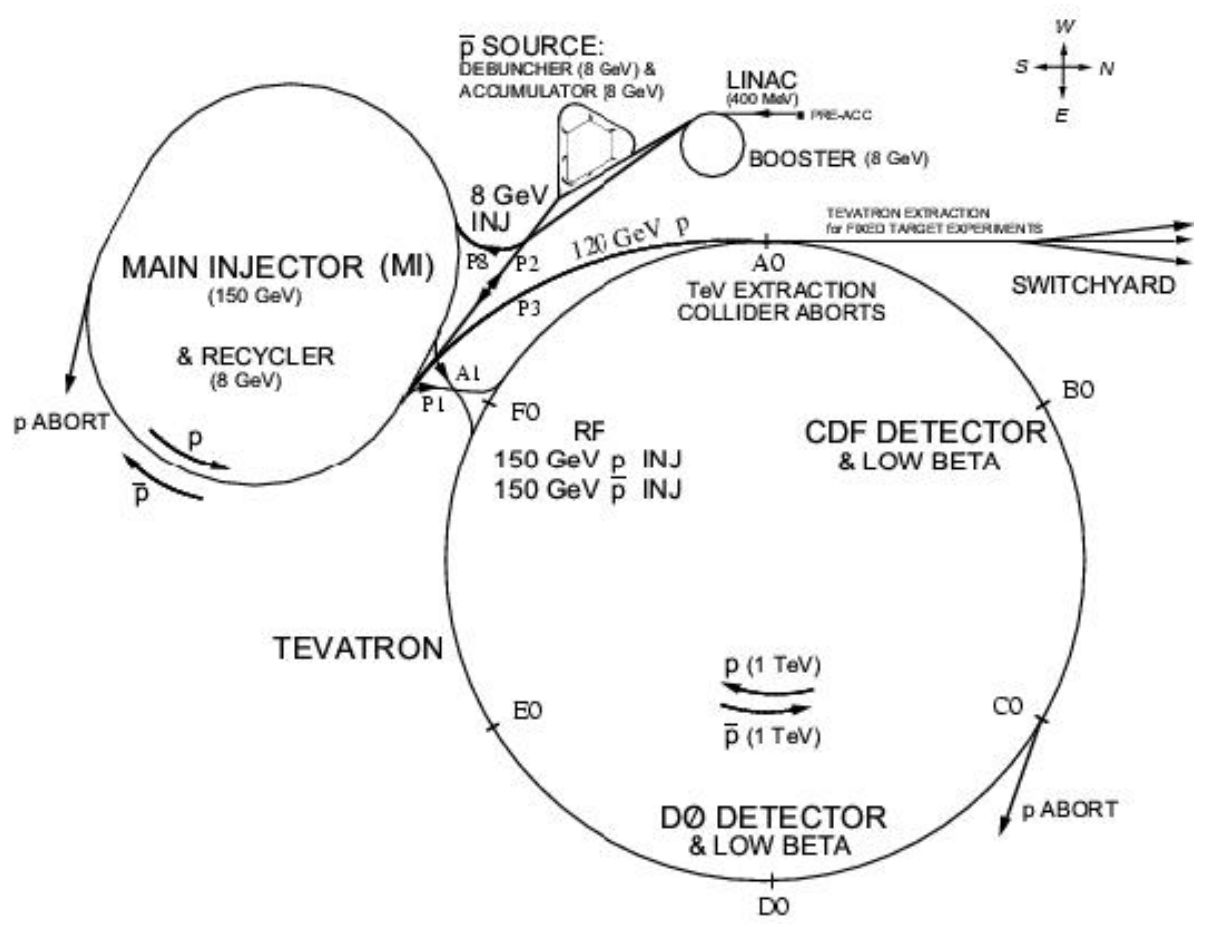

Fig. 2.2. Overview of the accelerator complex of Fermilab

are selected and transfered to the Debuncher ring where their momentum spread is reduced. The antiproton bunches are collected and stacked in the Accumulator ring. It takes up to 12 hours to collect $10^{12}$ antiprotons per bunch because their production rate is about 20 antiprotons in every million protons. The antiproton bunches are transfered back to the Main Injector where they are also accelerated to $150 \mathrm{GeV}$. In the beginning of each store, 36 proton and antiproton bunches are injected into the Tevatron and accelerated to the final energy of $0.98 \mathrm{TeV}$.

The proton and antiproton bunches are circulated in opposite directions in the Tevatron. There are points at sections B0 and D0 (Figure 2.2) where the two beams are crossed and proton-antiproton collisions can occur. A wide variety of particles are produced in inelastic collisions. These particles are detected by the two general purpose detectors called the Collider Detector at Fermilab (CDF) and D0. The latter one received its name after the point at which it is located in the Tevatron ring. 


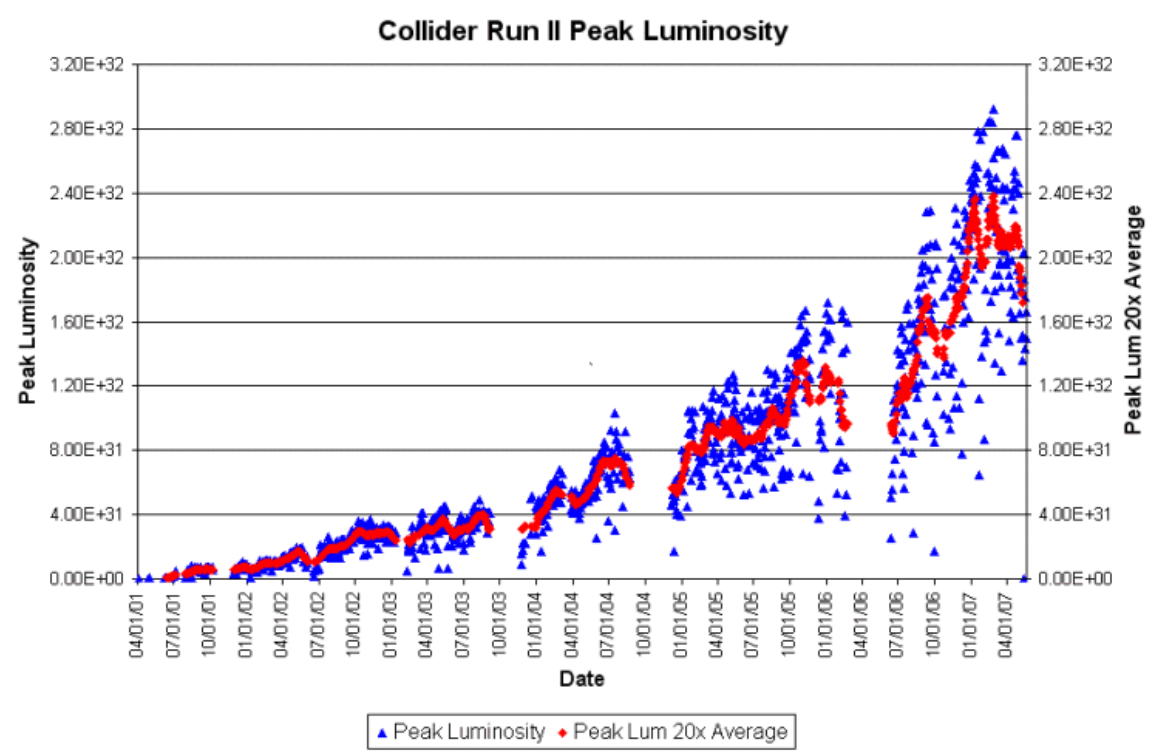

Fig. 2.3. Peak instantaneous luminosity in the beginning of the Run II stores at the Tevatron

\subsection{Luminosity}

The collision rate of protons and antiprotons are quantified by the instantaneous luminosity $(\mathcal{L})$. The luminosity is measured in units of $\mathrm{cm}^{-2} \mathrm{~s}^{-1}$ and can be calculated with the following formula:

$$
\mathcal{L}=\frac{f_{r} N_{B} N_{p} N_{\bar{p}}}{2 \pi\left(\sigma_{p}^{2}+\sigma_{\bar{p}}^{2}\right)} F\left(\frac{\sigma_{l}}{\beta^{*}}\right)
$$

where $f_{r}$ is the revolution frequency, $N_{B}$ is the number of bunches, $N_{p}$ and $N_{\bar{p}}$ are the number of protons and antiprotons per bunch, $\sigma_{p}$ and $\sigma_{\bar{p}}$ are the dispersion of the beam sizes at the interaction point [16]. $F$ is a form factor which corrects for the bunch shape and depends on the ratio of the bunch length $\sigma_{l}$ to the beta function $\beta^{*}$ at the interaction point. The beta function is a measure of the beam width, and is proportional to the beam's $x$ and $y$ spread in phase space.

The instantaneous luminosity is a decreasing function of time, because the number of particles in the bunches is decreasing over many bunch crossings and the beam also loses focus. The peak luminosity in the beginning of each store as a function of time 


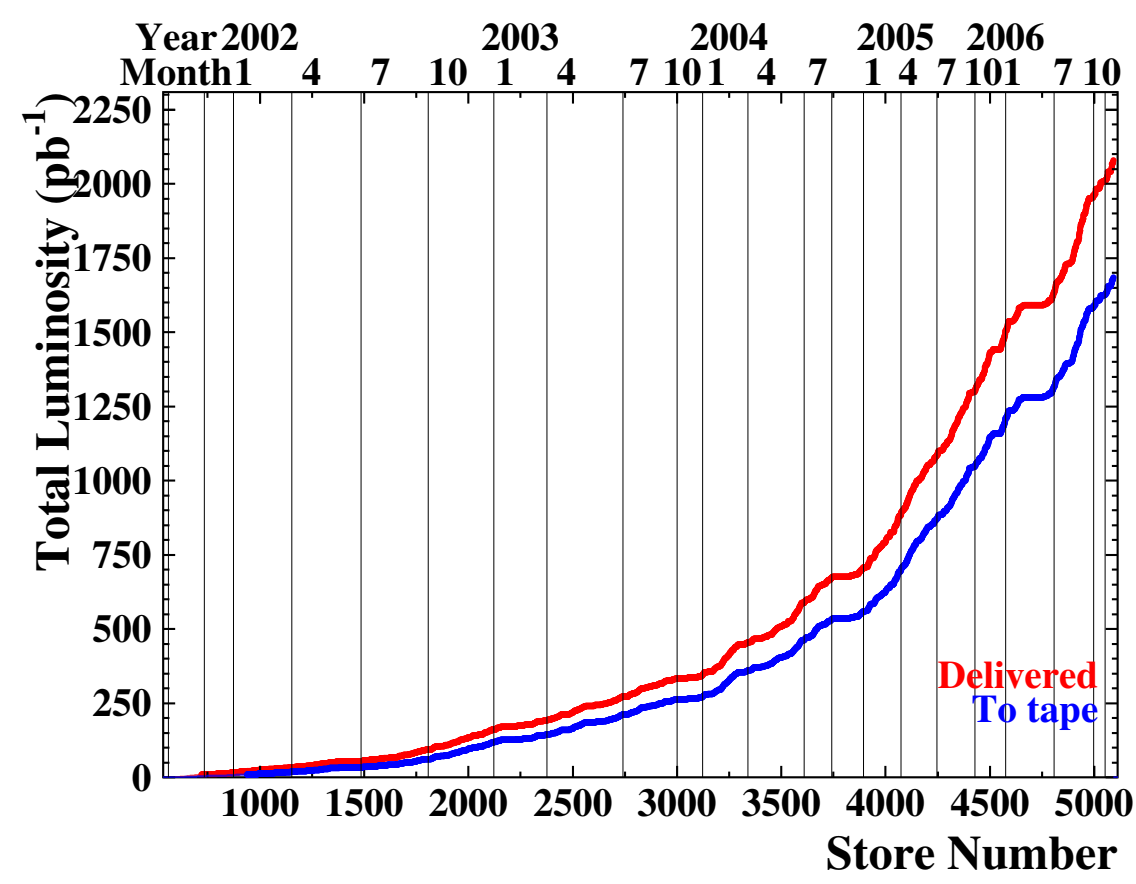

Fig. 2.4. Total integrated luminosity delivered by the Tevatron and recorded by the CDF experiment since 2001

is shown in Figure 2.3. There is a continuous effort to improve the instantaneous luminosity as a key to increasing the amount of data delivered by the Tevatron. The amount of data collected over a period of time is measured by the integrated luminosity $\int \mathcal{L}$ dt which is measured in inverse barn $\left(\mathrm{b}^{-1}\right)$, where $1 \mathrm{~b}^{-1}$ is $10^{24} \mathrm{~cm}^{-2}$. Figure 2.4 shows the profiles of the total delivered luminosity in different years of Run II of the Tevatron and how much of that was acquired by the CDF detector. The amount of data recorded by the experiments depends on the data taking efficiency. In order to maintain a high efficiency and record most of the events of interest, the online electronics often needs revision. The method that is used to measure luminosity is explained in Section 2.3.6. 


\subsection{The CDF Detector}

The first CDF detector was commissioned in 1987. It collected $\sim 110 \mathrm{pb}^{-1}$ data during the Run I period between 1992 and 1995. The typical instantaneous luminosity towards the end of Run I was $16 \times 10^{30} \mathrm{~cm}^{-2} \mathrm{~s}^{-1}$. Both the detector and the Tevatron underwent major upgrades between 1997 and 2001 [18] [19]. The main purpose of the upgrades was to increase the luminosity delivered by the accelerator and thus the amount of collision data acquired by the detectors. The larger luminosity was achieved by increasing the beam crossing rate so that the number of bunches increased from 6 to 36 and the bunch spacing decreased from $3.5 \mu$ s to 396 ns. The new collider required improved detectors to be adapted to the higher collision rate and new time structure. CDF II was commissioned in the beginning of Run II in 2001. Currently, the initial instantaneous luminosity is often above $300 \times 10^{30} \mathrm{~cm}^{-2} \mathrm{~s}^{-1}$. The design goal for the integrated luminosity in Run II is $8 \mathrm{fb}^{-1}$.

The CDF detector is a general purpose detector designed to study $p \bar{p}$ collisions at the Tevatron. The detector components are arranged in cylindrical shape. The position of the subdetectors are described in cylindrical coordinates $(r, \phi, z)$. The origin is fixed in the geometric center of the detector. The z-axis points along the beam in the direction of the protons.

The interaction of the incoming particles occur close to the origin. The outgoing particles are described in spherical coordinates. The z-axis is replaced by the polar angle $\theta$. In the event reconstruction, the transverse momentum of particles $p_{T}=$ $|\mathbf{p}| \sin \theta$ is measured in the transverse plane, and their direction is given by the pseudorapidity $\eta$. At high energies where the rest mass of the particle can be neglected $\left(p_{T}>>m\right)$ the pseudo-rapidity is defined as

$$
\eta \equiv-\ln \tan \left(\frac{\theta}{2}\right) .
$$

which is a good approximation to the rapidity

$$
y=\frac{1}{2} \ln \left(\frac{E+p_{z}}{E-p_{z}}\right) .
$$




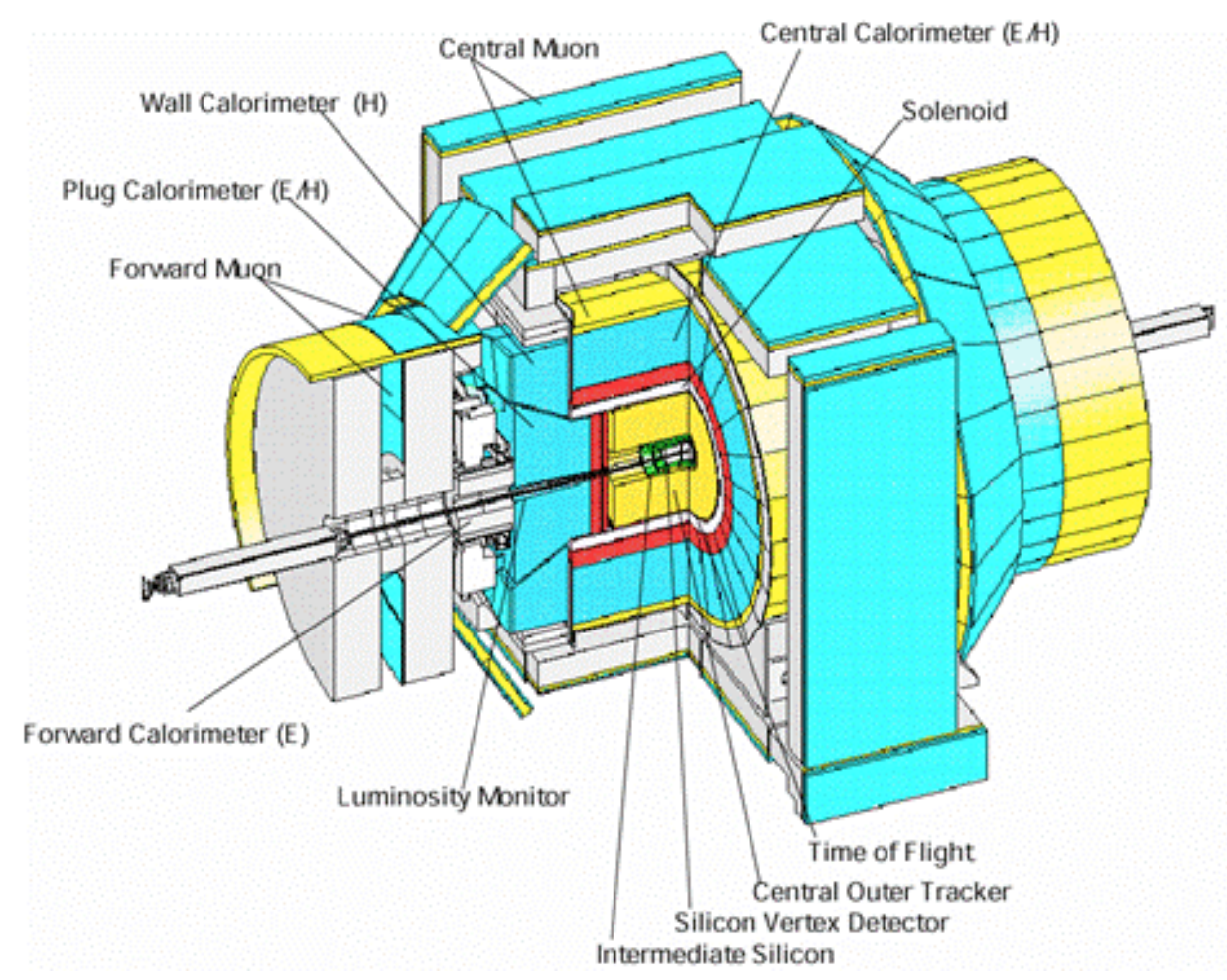

Fig. 2.5. Isometric view of the Collider Detector at Fermilab

where $E$ is the particle's energy and $p_{z}$ is its momentum along the z-axis. In this mapping, the $r-\phi$ plane corresponds to $\eta=0$.

A solid cutaway view of the detector is shown in Figure 2.5. In the center of the CDF detector is a charged particle tracking system enclosed by a superconducting solenoid. The solenoid is $1.5 \mathrm{~m}$ in radius and $4.8 \mathrm{~m}$ in length. It generates a $1.4 \mathrm{~T}$ magnetic field parallel to the beam axis. Outside the solenoid is the calorimeter system which is surrounded by the muon detectors.

\subsubsection{Tracking System}

The innermost part of the CDF detector is the integrated tracking system (Figure 2.6) which is composed of a superconducting solenoid magnet, multi-layer silicon mictrostrip detectors, and an open-cell wire drift chamber. It is used to reconstruct trajectories of charged particles and precisely measure their momenta. The recon- 


\section{CDF Tracking Volume}

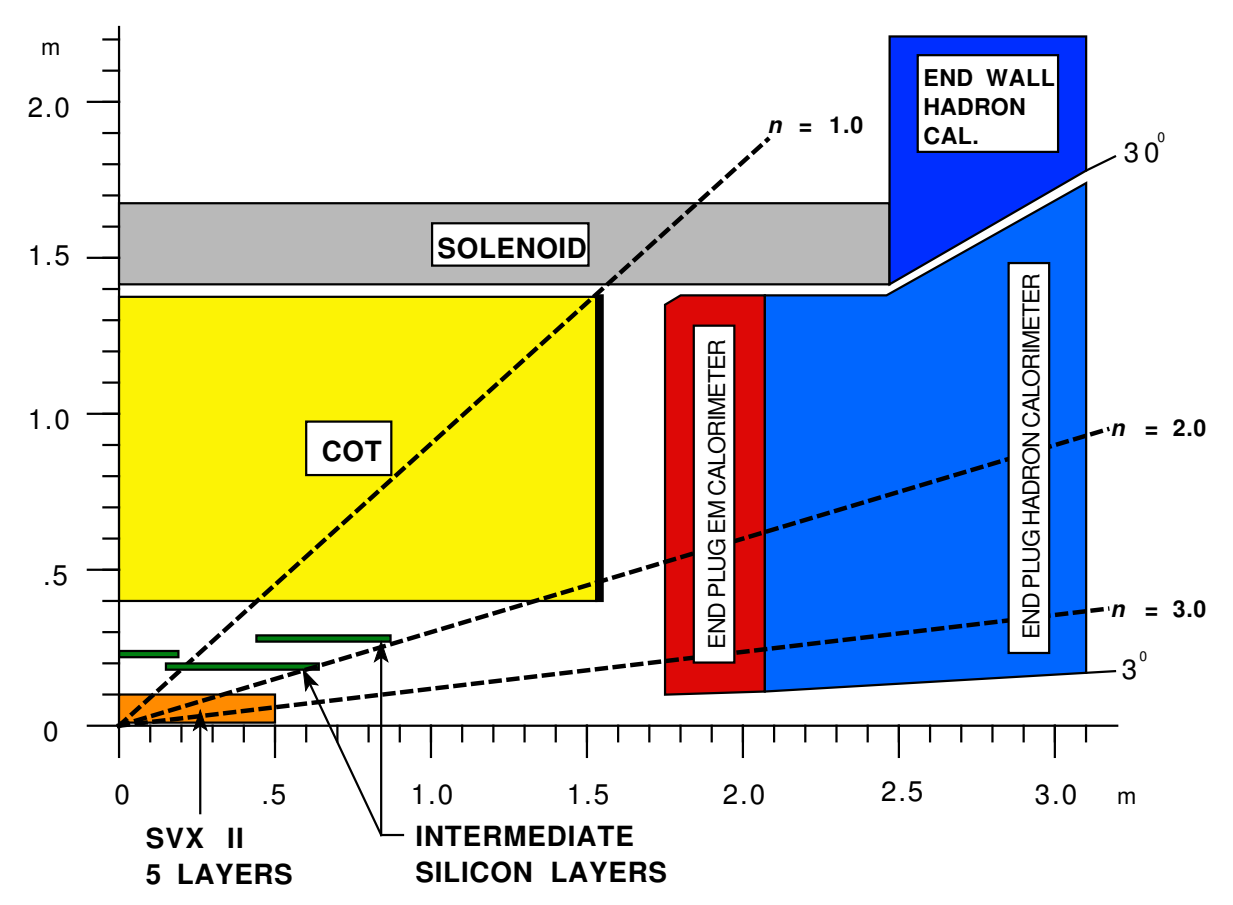

Fig. 2.6. Longitudinal view of the Tracking System in the CDF detector

structed particle trajectories are called tracks. The vertex where the $p \bar{p}$ interaction takes place is reconstructed in events with multiple tracks. Secondary vertices displaced with respect to the primary vertex are found in decays of heavy flavor quarks. Tracking is also used for identifying particles by measuring their energy loss due to ionization (using $d E / d x$ ) and their charge. Electrons, muons, and photons are identified by combining tracking information with data obtained from the other subdetectors. This information is available both offline and online.

\section{Inner Tracker}

The Inner Tracker consists of three parts: Layer 00 (L00) [20,21], the Silicon Vertex Detector (SVX II), and the Intermediate Silicon Layers (ISL) [22,23]. All the three sub-detectors are constructed from silicon microstrip detectors arranged in cylindrical barrels around the beam pipe as shown in Figure 2.7. Silicon microstrip detectors are 


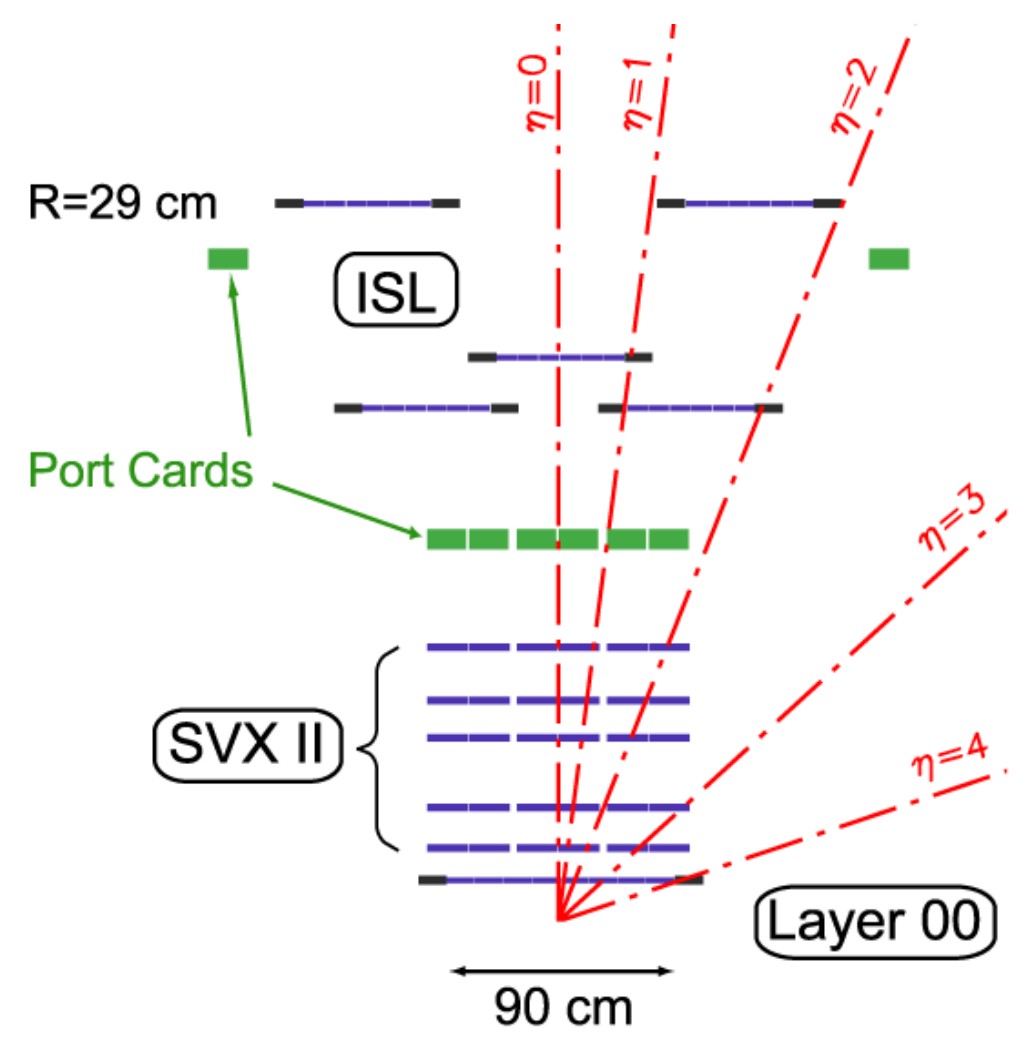

Fig. 2.7. Configuration and $\eta$-coverage of the three components of the inner tracker: L00, SVX II, and ISL

built on thin silicon wafers that contain p-n junctions. The p-n junction is reversebiased which creates a charge depleted volume within the wafer. Charged particles passing through the depleted region create electron-hole pairs. The $\mathrm{p}$ or $\mathrm{n}$ side of the junction contains closely spaced conducting strips, which are used to measure the amount of charge induced by the deposited energy of the traversing particles. The energy needed for one electron-hole pair production in silicon is about $3 \mathrm{eV}$. Silicon detectors have better energy resolution compared to gaseous drift chambers where the ionization energy is much higher (around 10 to $15 \mathrm{eV}$ ) and spacial resolution due to the possibility of achieving smaller pitches.

Layer 00 consists of one layer of radiation-hardened strip detectors, that are mounted on the beam pipe surrounding it at $\sim 1.5 \mathrm{~cm}$ radius. Layer 00 only provides information in the $r-\phi$ plane. Its purpose is to improve the impact parameter 

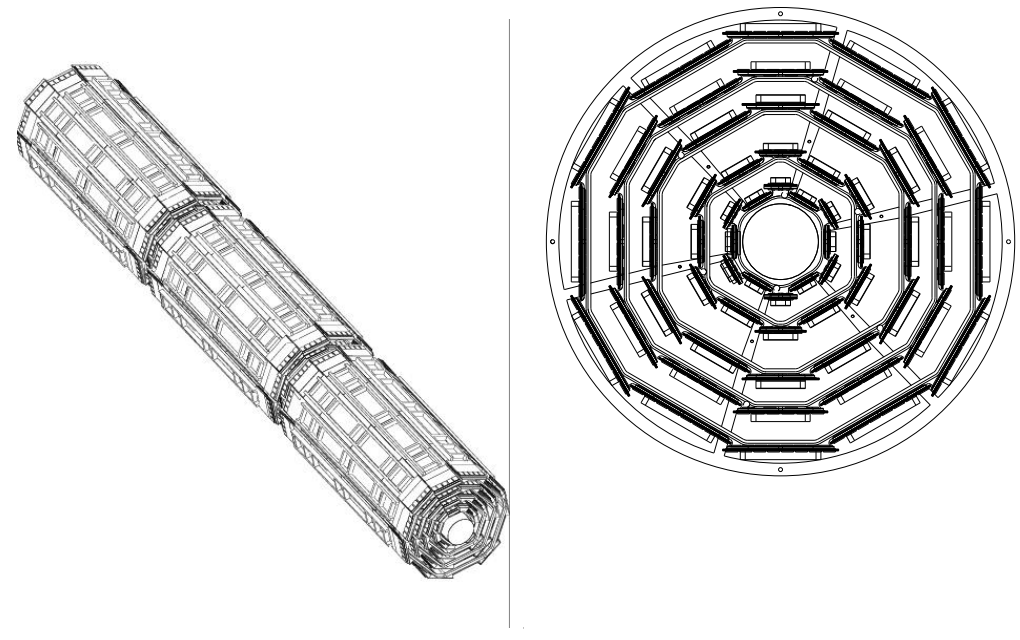

Fig. 2.8. Isometric and end-view of the CDF Silicon Vertex Detector

resolution and compensate for the degraded performance of the other silicon subdetectors due to radiation damage.

SVX II, shown in Figure 2.8, is the core of the silicon tracking system. It extends from $r=2.45 \mathrm{~cm}$ to $10.6 \mathrm{~cm}$. It is segmented into three barrels along the z-axis with a total length of $96 \mathrm{~cm}$ (about $2.5 \sigma$ of the luminous region) that covers $|\eta| \leq 2.0$ in pseudo-rapidity. Each barrel has five layers of double-sided silicon detectors. One side of the detectors measure position in $\phi$, while the other side in $z$. The strips on one side of all layers run along the z-axis, while on the other side they are perpendicular to the z-axis in three layers and tilted by $1.2^{\circ}$ w.r.t. the transverse direction on the other two. Four silicon wafers are mounted on light support structures called ladders. Twelve concentric ladders make a layer. Table 2.1 shows the parameters of the SVX II system. From the end-view in Figure 2.8, five layers form one of the twelve wedges. There are a total of 405,504 channels in the system.

ISL is the third and the outermost component of the Inner Tracker which serves as a link between the inner tracking region and the outer wire tracker. In the central region $(|\eta|<1.0)$, a single layer is located at a radius of $22 \mathrm{~cm}$. Two layers are placed at radii of $20 \mathrm{~cm}$ and $28 \mathrm{~cm}$ at $1.0 \leq|\eta| \leq 2.0$. In the ISL, only three double-sided 
Table 2.1

Parameters for the layout of sensors in various SVX-II layers.

\begin{tabular}{|lr|c|c|c|c|c|}
\hline Property & & Layer 0 & Layer 1 & Layer 2 & Layer 3 & Layer 4 \\
\hline \hline number of $\phi$ strips & 256 & 384 & 640 & 768 & 869 \\
number of $Z$ strips & & 256 & 576 & 640 & 512 & 869 \\
stereo angle & $\left({ }^{\circ}\right)$ & 90 & 90 & +1.2 & 90 & -1.2 \\
$\phi$ strip pitch & $(\mu \mathrm{m})$ & 60 & 62 & 60 & 60 & 65 \\
$Z$ strip pitch & $(\mu \mathrm{m})$ & 141 & 125.5 & 60 & 141 & 65 \\
active width & $(\mathrm{mm})$ & 15.30 & 23.75 & 38.34 & 46.02 & 58.18 \\
active length & $(\mathrm{mm})$ & 72.43 & 72.43 & 72.38 & 72.43 & 72.43 \\
\hline
\end{tabular}




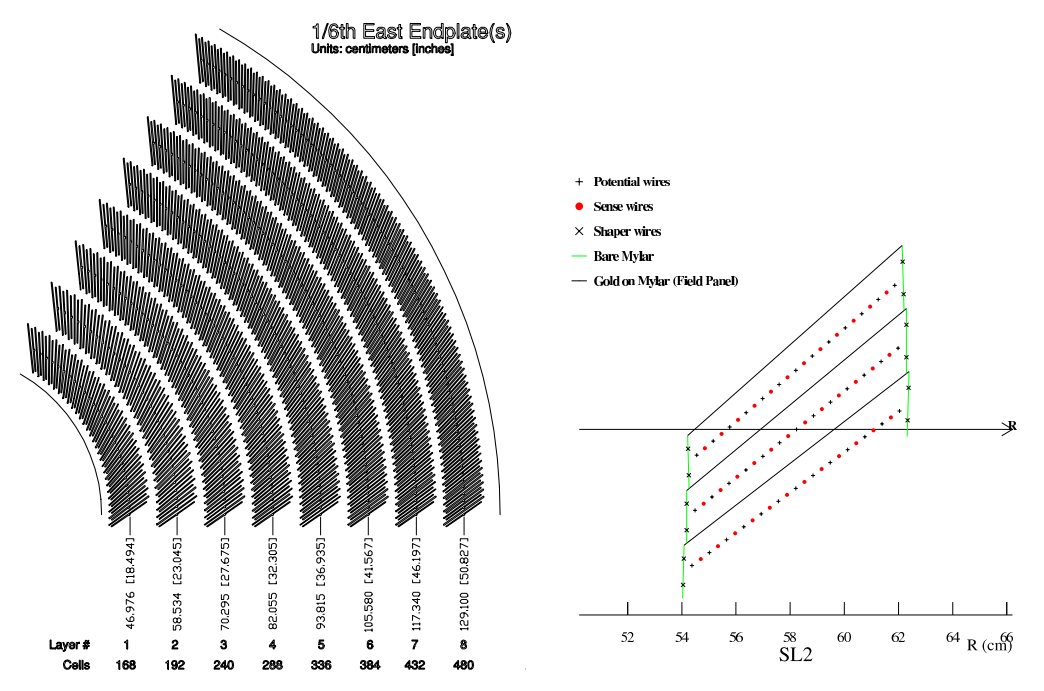

Fig. 2.9. End-view of a section in the Central Outer Tracker. Odd(even) numbered superlayers are small-angle stereo (axial). Configuration of the sense and potential wires in a COT cell

silicon sensors are mounted on one ladder. The position of a hit is measured in both $r-\phi$ and $z$ directions.

\section{Central Outer Tracker (COT)}

The Central Outer Tracker, shown in Figure 2.9, is a cylindrical open cell drift chamber that provides full tracking in the central region, $|\eta| \leq 1.0$ [24]. Its active region is $310 \mathrm{~cm}$ in the $z$ direction and extends between $44 \mathrm{~cm}$ and $132 \mathrm{~cm}$ in radius. The maximum acceptance reaches up to $|\eta| \leq 2.0$ with reduced tracking performance.

The COT is built of 8 cylindrical superlayers that are divided into cells in $\phi$ (Figure 2.9). The number of cells in the superlayer increases radially outwards such that it is proportional to the circumference corresponding to the radius (Table 2.2). Each cell contains 12 parallel uniformly spaced sense wires and 13 potential wires arranged alternately and sandwiched between two grounded (cathode) field sheets. The voltage on the sense wires are 2600-3000 Volts and 1000-2000 Volts on the potential wires. The chambers are filled with a mixture of Argon-Ethane gas bubbled through isopropyl alcohol. When traversing the chambers, charge particles ionize the gas. Electrons 


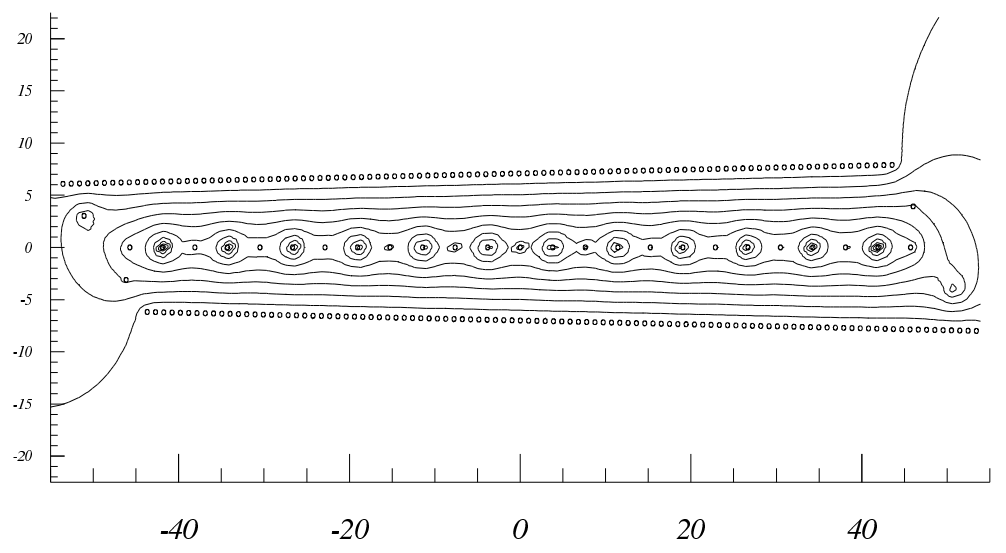

Fig. 2.10. Equipotential lines around the sense wires in a COT cell.

produced in the ionization drift towards the sense wires with a velocity of $50 \mu \mathrm{m} / \mathrm{ns}$. The electrons approaching the sense wires gain energy due to the logarithmically increasing potential and induce an avalanche of charges through secondary ionizations. The potential wires shape the electric field around the sense wires such that the field along the drift trajectories is nearly uniform (Figure 2.10). Since the COT chambers are located inside the detector magnet, the drifting electrons experience a Lorentz force that rotates their path. In order to compensate for this effect, the cells are tilted by $35^{\circ}$, thus the electron drift is approximately perpendicular to the wires. Wires in even (axial) superlayers run in the $z$ direction. Wires in odd (stereo) superlayers have a $\pm 3^{\circ}$ angle to the $z$-axis. The former ones provide tracking information in $r-\phi$, the latter ones in the $z$ direction.

The analog signals on the sense wires are read out by the ASDQ (Amplifier, Shaper, Discriminator with charge encoding) chip which provides input protection, amplification, pulse shaping, baseline restoration, discrimination and charge measurement. The leading edge provides information on the arrival time, and the pulse width is related to the amount of charge collected by the wire. After calibrating the width variations due to the COT geometry, path length of the particle, and the gas gain 
Table 2.2

COT Parameters

\begin{tabular}{|l|r|}
\hline Parameter & Value \\
\hline Gas & Ar/Et $/ \mathrm{CF}_{4}(49.5: 49.5: 1)$ \\
Max. Drift distance & $0.88 \mathrm{~cm}$ \\
Max. Drift Time & $177 \mathrm{~ns}$ \\
Lorentz Angle & $31^{\circ}\left(35^{\circ}\right.$ cell tilt $)$ \\
Drift Field & $1.9 \mathrm{kV} / \mathrm{cm}$ \\
Radiation Lengths & $1.7 \%$ \\
Total sense wires & 30,240 \\
Number of cells per SL & $168,192,240,288$, \\
& $336,384,432,480$ \\
Stereo Angle & $+2^{\circ}, 0^{\circ},-2^{\circ}, 0^{\circ}+2^{\circ}, 0^{\circ},-2^{\circ}, 0^{\circ}$ \\
\hline
\end{tabular}

difference for the 96 wires, the digital width that is related to the ionization energy loss $d E / d x$ is used for particle identification.

\subsubsection{Time of Flight Detector (TOF)}

In order to complement the particle identification capability of the tracking system, the TOF detector was added as part of the CDF Run II upgrade [25]. The TOF is located between the COT and the CDF solenoid at a radius of $140 \mathrm{~cm}$ also providing coverage in $|\eta| \leq 1$. It consists of 216 scintillator bars that measure the arrival time $t$ of a charged particle with respect to the collision time with a precision of 100 ps.

The mass of particles is computed as $m=\frac{p}{c} \sqrt{\frac{c^{2} t^{2}}{L^{2}}-1}$ that combines the TOF time with the momentum and path length measurement from the tracking. The Time 


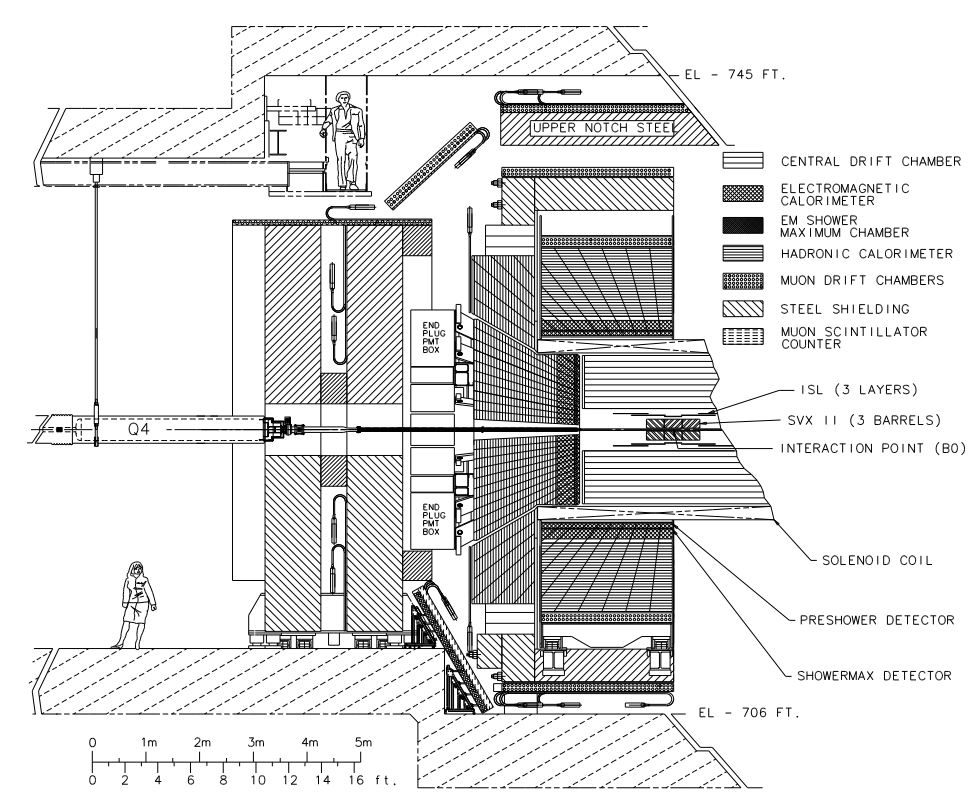

Fig. 2.11. Elevation view of the CDF detector

of Flight detector was not used in this analysis, because the high- $p_{T}$ objects of our interest such as jets and leptons are defined based on calorimeter information.

\subsubsection{Calorimeters}

The CDF calorimeter detectors measure energy of both charged and neutral particles produced in the $p \bar{p}$ collisions by absorbing their total energy. These measurements combined with the tracking information are used in particle identification. They can also be used for measuring transverse energy of weakly interacting particles, such as neutrinos, that escape detection by computing the imbalance in the total transverse energy.

A particle traversing the calorimeter interacts with the detector material and loses a fraction of its energy. This fraction depends on the particle type and its initial energy. Electrons above $100 \mathrm{MeV}$ lose their energy by photon radiation, or bremsstrahlung; whereas photons produce electron-positron pairs. Cascades of these two basic interactions form showers in the detector. The amount of energy deposited 
by these showers increases with the detector depth until the particles in the shower are not energetic enough for further particle multiplication (shower maximum). Beyond the shower maximum depth, the deposited energy per unit length gradually decreases. The distance over which a traversing electron loses on average $1-e^{-1}$ fraction of its energy in a given material is called radiation length $X_{0}$. For charged particles heavier than the electron, radiative energy losses start dominating at much higher energies that are proportional to the square of the ratio between the mass of the particle and the electron mass.

Hadrons interact with the detector matter mostly through inelastic collisions with the nuclei of the absorbing medium. Particles produced in the nuclear interactions can lose their energy by ionization or secondary nuclear interactions, therefore they also form showers. The nuclear interaction length $\lambda_{0}$ is defined as a mean free path for a hadron to undergo a nuclear inelastic interaction. Since the nuclear interaction crosssection is smaller than the electromagnetic, the interaction length is longer than the radiation length $X_{0}$. However, due to the higher multiplicity in nuclear interactions, the number of interaction lengths required for hadrons to lose their energy is fewer than the number of radiation lengths required for electrons or photons.

CDF uses scintillator sampling calorimeters divided to an inner electromagnetic and a larger outer hadronic section. Both calorimeters are segmented into projective towers (Figure 2.11). Each tower consists of alternating layers of passive absorber material and active scintillator tiles for shower sampling. The light from the scintillator plates is read out through wavelength-shifting (WLS) light guides by photo-multiplier tubes (PMT). The total energy resolution of the measurement $(\sigma / E)^{2}=\left(\sigma_{1} / \sqrt{E}\right)^{2}+\left(\sigma_{2}\right)^{2}$ depends on statistical sampling fluctuations, the photostatistics of the PMTs which are inversely proportional to the square root of the incident energy, and a constant term due to non-uniform response of the calorimeter, calibration errors and noise in the electronics [19].

The CDF calorimeter system provides azimuthal coverage and up to 3.6 in $|\eta|$. It includes the Central Electromagnetic Calorimeter (CEM) and the Hadronic Calorime- 
ter (CHA) in the $|\eta|<0.9$ region (Figure 2.11), the Endwall Hadronic Calorimeter (WHA) in $0.9<|\eta|<1.3$, and the electromagnetic and hadronic plug calorimeters PEM and PHA in $1.1<|\eta|<3.6$ (Figure 2.12). In the forward region, there is only an electromagnetic calorimeter, called the Miniplug (MNP), which is not used in this search. Table 2.3 summarizes the calorimeter detectors, their coverage and energy resolution.

\section{Central Calorimeters}

The central calorimeters, CEM, CHA, and WHA, are inherited from Run I. The front-end electronics was upgraded for Run II in order to facilitate data acquisition with shorter bunch-crossing. Their structure is also cylindrically symmetric. The detectors are divided into two halves along the $z$-direction at $|\eta|=0$ where there is a small uninstrumented region (crack). The CEM is closer to the interaction point and covers the region $|\eta|<1.1$ on both sides, CHA and WHA are outside the CEM and provide coverage in $|\eta|<0.9$ and $0.9<|\eta|<1.3$, respectively. Each calorimeter is divided into projective towers of size $15^{\circ}$ in $\phi$ and 0.11 in $\eta$.

The CEM calorimeter is made of alternate layers of $0.5 \mathrm{~cm}$ plastic scintillator plates and $0.32 \mathrm{~cm}$ thick lead absorbers. The relative energy resolution of the detector is $(13.5 \% / \sqrt{E}) \oplus 2 \%$ for a single electron or photon. A combined strip/wire gas proportional chamber called Central Electromagnetic Shower maximum detector (CES) is embedded in CEM at about 6 radiation lengths, which measures the position and shape of EM showers in both $r-\phi$ and $z$. The CHA and WHA calorimeters use $1 \mathrm{~cm}$ thick scintillator layers sandwiched between $2.5 \mathrm{~cm}$ and $5 \mathrm{~cm}$ thick steel layers.

\section{Plug Calorimeters}

The plug calorimeters, PEM and PHA, were a major part of the Run II upgrade program. Figure 2.12 shows the cross-section of the upper part of the plug calorimeters. Their coverage extends in the range $1.1<|\eta|<3.6$. The tower segmentation is the same as in the central calorimeters in $\eta$, but is finer by a factor of $2\left(7.5^{\circ}\right)$ in the 


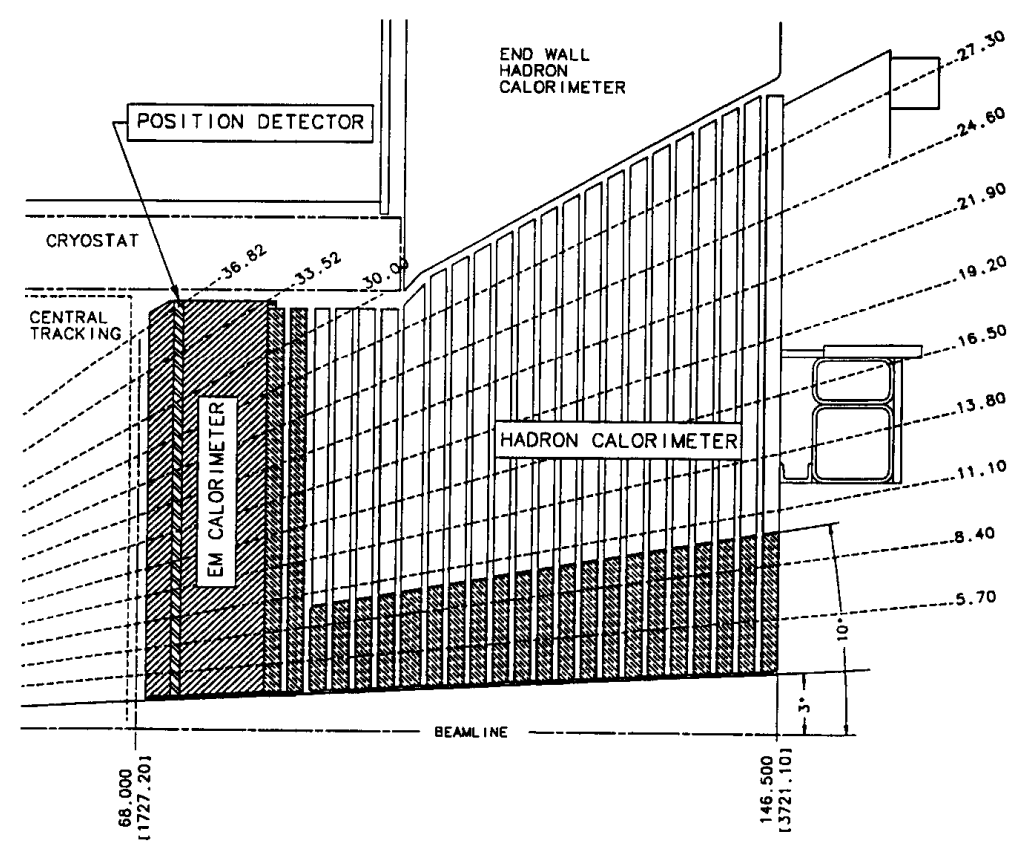

Fig. 2.12. $R-z$ cross-sectional view of the new Plug Calorimeter

azimuthal direction for $|\eta|<2.11$. The thickness of the scintillator and lead tiles is $0.4 \mathrm{~cm}$ and $0.45 \mathrm{~cm}$ in PEM. About 6 radiation lengths deep in the Plug Calorimeters is the Plug Shower maximum detector (PES). In WHA, the scintillator layers are 0.6 $\mathrm{cm}$ and the iron layers are $5 \mathrm{~cm}$ thick.

\subsubsection{Muon Detector}

Muons travel through larger amounts of material before losing their energy, therefore they are not identified in the calorimeters. The detectors dedicated to measuring muons are located the farthest away from the interaction point. There are four systems of wire chambers covered by scintillators for muon detection that provide a coverage up to $|\eta|<1.5$. 
Table 2.3

The energy resolution of the EM calorimeter is given for a single incident electron and that of the hadronic calorimeter for a single incident pion. $\lambda_{0}$ refers to the interaction length and $X_{0}$ to the radiation length.

\begin{tabular}{|l|r|c|r|}
\hline Calorimeter & Coverage & Thickness & Energy resolution \\
\hline CEM & $|\eta|<1.1$ & $18 X_{0}$ & $\frac{13.5 \%}{\sqrt{E_{T}}} \oplus 2 \%$ \\
CHA & $|\eta|<0.9$ & $4.5 \lambda_{0}$ & $\frac{75 \%}{\sqrt{E_{T}}} \oplus 3 \%$ \\
WHA & $0.7<|\eta|<1.2$ & $4.5 \lambda_{0}$ & $\frac{75 \%}{\sqrt{E_{T}}} \oplus 3 \%$ \\
\hline PEM & $1.1<|\eta|<3.6$ & $21 X_{0}, 1 \lambda_{0}$ & $\frac{16 \%}{\sqrt{E}} \oplus 1 \%$ \\
PHA & $1.2<|\eta|<3.6$ & $7 \lambda_{0}$ & $\frac{80 \%}{\sqrt{E}} \oplus 5 \%$ \\
\hline
\end{tabular}


There are three detectors in the central region: Central Muon Detector (CMU), Central Muon Upgrade (CMP) and Central Muon Extension (CMX). The central calorimeter and the return yoke of the magnet serve as hadron absorbers, furthermore, a $60 \mathrm{~cm}$ layer of steel is placed in front of the CMP. The CMU consists of 144 modules with 16 rectangular cells per module. The cells in a module are stacked four layers deep in the radial direction with a small offset between the first and third and the second and fourth layers. Wires in cell pairs are read out together with a TDC to measure $\phi$ coordinates and by ADCs on each end to measure the muon's $z$-coordinate via charge division. The CMP is located outside the CMU. Both detectors identify muons up to $|\eta|<0.6$. The CMP chambers are rectangular, single-wire drift tubes configured in four layers with alternate half-cell staggering. There is a layer of scintillator counters on the outside of the CMP called CSP. The third central muon detector, the CMX, extends the coverage in $|\eta|$ from 0.6 to 1 . It consists of $15^{\circ}$ conical sections of $12 \mathrm{drift}$ tubes placed in four layers. The CMX is also covered by a scintillation counter called CSX.

The forward region of the system is called the Intermediate Muon Detector (IMU) that provides muon identification between 1.0 and 1.5. It is made of muon chambers (BMU) and scintillation counters (BSU) in a cylindric shape aligned parallel to the beam line.

In this analysis, the muon system is not used, because lepton identification is done based on tracking and calorimeter information. This provides a lower purity but higher efficiency in muon identification. More information on the muon system can be found in [26] and [27].

\subsubsection{Cherenkov Luminosity Counters}

The luminosity measurement is performed by the Cherenkov Luminosity Counters (CLC) located in the very forward regions $(3.75<|\eta|<4.75)$ of the CDF detector on both sides $[28,29]$. The CLC consists of three times 16 conical shaped gas-filled 


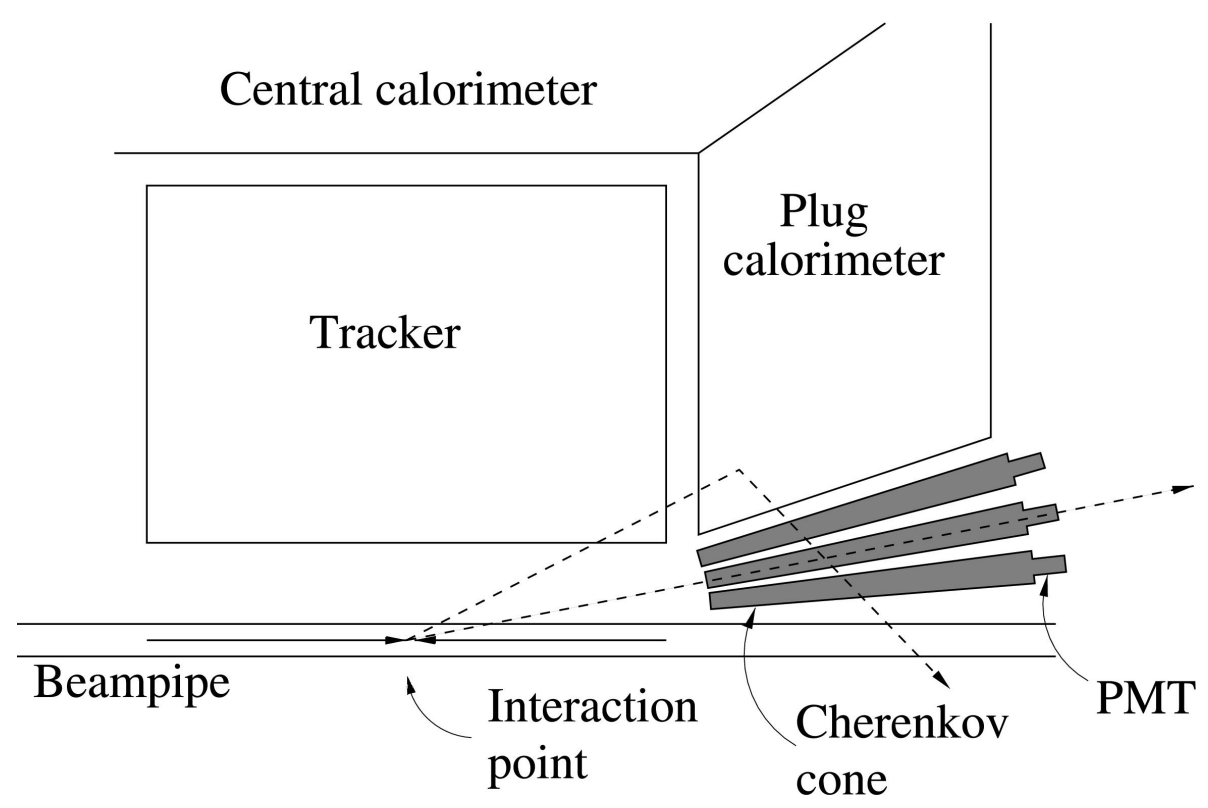

Fig. 2.13. Location of the Cherenkov Luminosity Counters at small angles in the forward regions

cherenkov counters arranged in three concentric cones. The innermost counter is $110 \mathrm{~cm}$ long, the outer two are $180 \mathrm{~cm}$ long, all pointing at an angle towards the interaction point (Figure 2.13). The entire assembly is placed in an aluminum vessel filled with isobutane gas at 15 times the atmospheric pressure.

In an optically dense material, charged particles traveling faster than the speed of light in that medium and emit Cherenkov radiation. The condition for this radiation is that $\beta=\frac{v}{c}>\frac{1}{n}$ where $n$ is the index of refraction of the medium. The angle of the radiation, called the Cherenkov angle, is $\theta_{C}=\arccos \left(\frac{1}{n \beta}\right)$ with respect to the velocity of the incident particle. The isobutane has a refraction index of 1.00215 which corresponds to a $\theta_{C}=3^{\circ}$ angle. The momentum threshold for the radiation is 9.3 MeV/c for electrons and $2.6 \mathrm{GeV} / \mathrm{c}$ for pions. The Cherenkov light is detected with $2.5 \mathrm{~cm}$ diameter photomultiplier tubes. 


\subsubsection{Luminosity Measurement}

The luminosity was defined in Section 2.2. It provides a measure for the amount of collision data that is acquired by the CDF detector. The luminosity is recorded by the CLC detector after counting the number of interactions per bunch crossing $\mu$ and calculating it from the following equation:

$$
\mu \cdot f_{B C}=\sigma_{i} \cdot \mathcal{L}
$$

where $f_{B C}$ is the bunch crossing frequency of $1.7 \mathrm{MHz}$ for $36 \times 36$ bunch operations, $\sigma=60.7 \mathrm{mb}$ is the inelastic cross-section at the Tevatron, and $\mathcal{L}$ is the instantaneous luminosity. The hit counting method works up to about $200 \times 10^{32} \mathrm{~cm}^{-2} \mathrm{~s}^{-1}$ where on average $6 p \bar{p}$ interactions are expected; however, at very high instantaneous luminosity the CLC saturates due to the increased occupancy.

Another method for measuring the luminosity is based on counting the empty bunch crossings. The number of interactions $n$ per bunch crossing follows Poisson statistics and is computed by:

$$
\mathcal{P}(n)=\frac{\mu^{n} e^{-\mu}}{n !}
$$

The probability for empty crossings is

$$
\mathcal{P}(0)=e^{-\mu}
$$

By measuring the fraction of empty bunch crossings, $\mu$ can be calculated. This method has limited use at high luminosity due to the small $\mathcal{P}(0)$ and the pile-up in the detector.

The systematic uncertainty of the luminosity measurement is $6 \%$, which follows from the $4.4 \%$ uncertainty on the detector acceptance and a $4 \%$ uncertainty on the total $p \bar{p}$ inelastic cross-section.

\subsubsection{Data Acquisition and Trigger System}

The Tevatron Run II bunch spacing is $396 \mathrm{~ns}$ which corresponds to $2.53 \mathrm{MHz}$ bunch-crossing rate. The actual average interaction rate is somewhat lower, $1.7 \mathrm{MHz}$. 
The number of interactions per bunch crossing varies with the instantaneous luminosity. In the first half of the $1 \mathrm{fb}^{-1}$ data used in this analysis, the typical luminosity was around $40 \times 10^{30} \mathrm{~cm}^{-2} \mathrm{~s}^{-1}$ with one interaction per crossing in the majority of the events. In later runs, the CDF detector often experienced luminosity around $200 \times 10^{-30} \mathrm{~cm}^{-2} \mathrm{~s}^{-1}$ with about 6 interactions per bunch crossing. The typical size of a data event is $250 \mathrm{kB}$. The products of these three factors, well over $400 \mathrm{~GB}$ per second, determine the rate at which the CDF detector would need to store data arriving from the subdetectors if every event needed to be recorded. The current maximum storage rate however is around $20-30 \mathrm{MB} / \mathrm{s}$. Fortunately, not all these events are actually interesting for physics analyses; most of them are "minimum bias" events which are inelastic scatterings with no significant momentum transfer. The crosssections of events with interesting physics are several orders of magnitude smaller. As an extreme example, the Higgs production cross-section expected in our analysis is $10^{-12}$ times the total $p \bar{p}$ cross-section of $60.7 \mathrm{mb}$ at the Tevatron. Therefore, most of the events must be discarded while the interesting ones must be identified in the time between two collisions (online).

The categorization of events is done by dedicated fast online electronics, called the Trigger System. The data flow in the CDF detector is illustrated in Figure 2.14. The trigger system is composed of three levels, where each level of electronics performs a slower but more accurate event reconstruction and decision making based on a set of predefined conditions. Level 1 (L1) and Level 2 (L2) use custom hardware to do limited event reconstruction with some programmability at Level 2. (Note that the currently ongoing L2 Calorimeter upgrade [32] will tremendously improve the trigger system.) The event information flows through the L1 storage pipeline while the decision is made in the Level 1 trigger. The L1 trigger already discards about $97 \%$ of the events, the rest are handed to the Level 2 buffers that can hold up to 4 events at a time while the $\mathrm{L} 2$ electronics evaluates them. The combined L1+L2 rejection factor is about $2 \times 10^{4}$. Level 3 (L3) performs full event reconstruction in the Event Builder using an online computer farm that provides information with nearly offline 


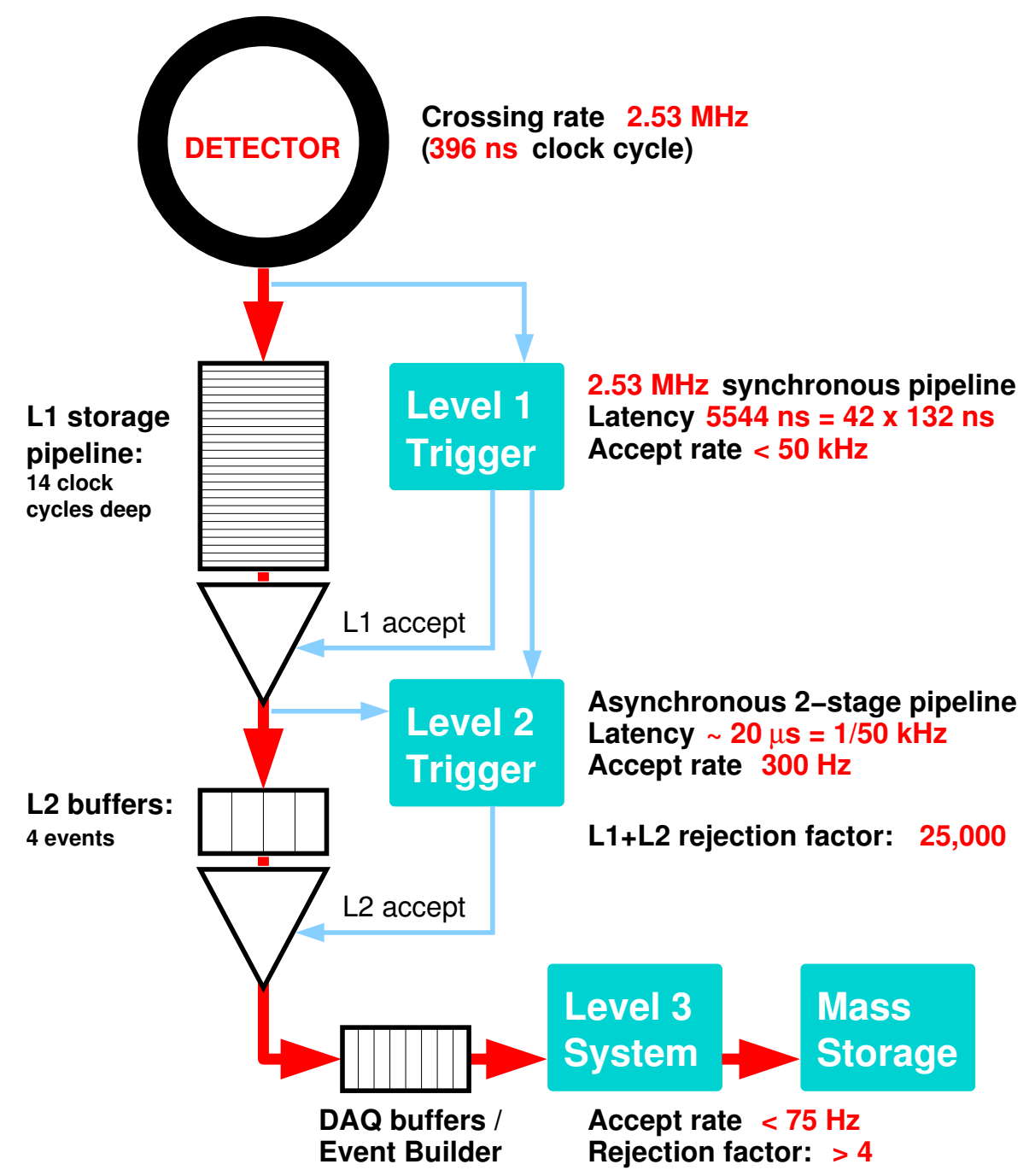

Fig. 2.14. Data-flow in the CDF II data acuisition system

accuracy and has an acceptance rate of about 75 events per second. When L3 accepts an event, it gets written to the mass storage. The list of conditions checked upon at each level is called the trigger path.

A block diagram of the trigger system is shown in Figure 2.15. The Level 1 trigger receives information about events from the calorimeters, the muon detector, the CLC, and the COT tracking through the eXtremely Fast Tracker (XFT) [30,31]. A limited group of selection requirements, called primitives, are tested. The L1 conditions in a trigger path are composed of these primitives connected by logical AND operations 
and are evaluated in the global L1 decision maker. There are about 120 trigger paths defined at Level 1 some of them sharing some primitives with other triggers (i.e. using the same selection threshold), but all designed to perform selection for a specific purpose. If an event satisfies any of them, it gets transfered to Level 2.

The Level 2 trigger consists of several asynchronous subsystems which provide input to programmable L2 processors. Events received from L1 are stored in one of four buffers. If the buffers get filled up at any time, the following events get lost (deadtime losses). Currently, the increased instantaneous luminosity in the beginning of the stores at the Tevatron induces problems at the Level 2 output which necessitates the upgrade of the L2 Calorimeter triggers [32]. The Level 2 decisions are made based on:

- L2 cluster finder (L2CAL). The L2 cluster finder uses a recursive algorithm (with the picturesque name PacMan) that combines adjacent trigger towers over a $1 \mathrm{GeV}$ threshold starting from a seed tower of minimum $3 \mathrm{GeV}$. This algorithm does not limit the size of a cluster, therefore it becomes problematic in high occupancy events and it is not entirely compatible with the offline clustering scheme. A more compatible and robust algorithm is being developed for the L2 calorimeter trigger upgrade [32].

- Shower Maximum data. The shower maximum detectors provide spatial resolution for electron and photon showers in the calorimeters with better than the cluster location. It is also matched to the tracking information.

- Silicon Vertex Tracker (SVT) [33] [34]. The SVX information is combined with the Level 1 XFT tracking. The $\phi$ angle, limited stereo information, $p_{T}$, and impact parameter of the tracks are calculated with precision comparable to the offline reconstruction. It is possible to construct triggers that select b-jets at Level 2 based on the impact parameter of tracks within the jets.

- Muon detector. The muon trigger combines information arriving from the Muon system with tracking information. 


\section{RUN II TRIGGER SYSTEM}

Detector Elements

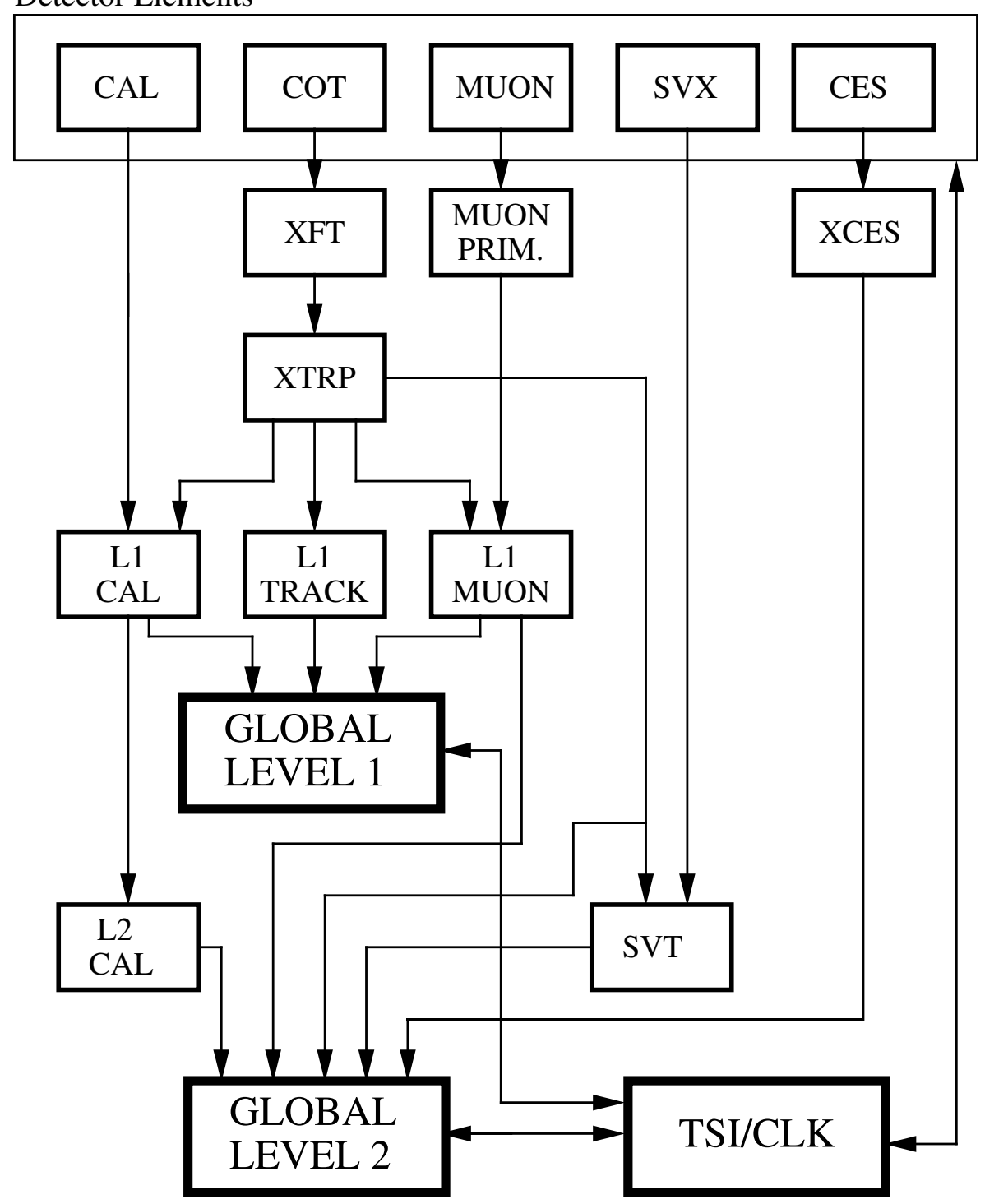

PJW 9/23/96

Fig. 2.15. Block diagram of the CDF Run II trigger system

The final decision in a trigger path is made at Level 3 [35]. The definition of the trigger path used in this analysis is given in Chapter 4. Since the observables that are 
used in the trigger system are not the same as the ones used for physics analysis (see Chapter 3), a separate study must be performed in order to measure the efficiency of the trigger selections with respect to the offline selections. This is also discussed in Chapter 4. 


\section{EVENT RECONSTRUCTION}

We search for the Higgs boson in events with missing transverse energy, two heavy flavor jets, and no leptons. Definition and description of the relevant observables are given in this chapter including jets, missing energy, charged particle tracks, b-jet finding methods and lepton identification for electron and muon vetos.

\subsection{Jets}

Collision events that trigger the detector contain one or more hard scattering processes in which a parton from the proton interacts with a parton from the antiproton. We are interested in detecting the products of hard interactions. Light particles such as electrons and muons are stable or have long lifetime and reach the subdetectors designed for their identification. Quarks and gluons, however, participate in more complex processes. First they undergo a process called fragmentation where they create partons via a cascade of gluon emissions and decays. The fragmentation continues until the momentum square of the partons is at the order of the infrared cut-off scale. Partons then form colorless hadrons in a process called hadronization. The non-stable hadrons decay to stable particles which reach the detector material. The showers of particles appear as clusters of energy deposited in localized areas of the calorimeter, called jets.

\subsubsection{Jet Reconstruction}

There are several algorithms developed for calorimeter jets. Some algorithms may also incorporate tracking information in searching for charged jets or in measuring 
their transverse momenta. The jet identification algorithm used in this search is called JETCLU which relies only on calorimeters [36].

The jets are defined as towers in circular regions of the $\eta-\phi$ plane, called cones, with radius 0.4 :

$$
\Delta R=\sqrt{\left(\Delta \eta_{i}\right)^{2}+\left(\Delta \phi_{i}\right)^{2}}<0.4
$$

where $\Delta \eta_{i}=\eta_{\text {cent }}-\eta_{i}$ and $\Delta \phi_{i}=\phi_{\text {cent }}-\phi_{i}$ are differences between the $E_{T}$-weighted average of the tower locations (centroid) and the $i^{\text {th }}$ tower location in pseudo-rapidity and azimuthal angle.

The algorithm starts searching for towers with $E_{T}>1 \mathrm{GeV}$, where

$$
E_{T}=E^{e m} \sin \theta^{e m}+E^{h a d} \sin \theta^{h a d}
$$

$\theta^{\text {em/had }}$ is the polar angle in the detector's coordinate system with origin placed at the highest $p_{T}$ vertex in the event. Then preclusters are created by grouping adjacent towers within the cone radius proceeding from the highest energy tower to the lowest one. One tower is assigned to only one precluster. In the next step, the centroids of the preclusters are calculated, and new 0.4 radius cones are defined including towers with at least $100 \mathrm{MeV}$. If the centroid of a new cluster changes, the cone is redefined and new towers are added iteratively (but not taken away). When a stable solution is found, overlaps between clusters are removed by either combining or separating contiguous clusters, and jets are defined.

A momentum four-vector is assigned to every electromagnetic and hadronic tower in a jet assuming zero mass, 3-D direction pointing from the event vertex to the shower maximum detector within the tower, and raw energy. The four-momentum of the jet is computed from its towers:

$$
\begin{gathered}
E=\sum_{i=1}^{N_{\text {towers }}}\left(E_{i}^{e m}+E_{i}^{\text {had }}\right), \\
p_{x}=\sum_{i=1}^{N_{\text {towers }}}\left(E_{i}^{e m} \sin \theta_{i}^{e m} \cos \phi_{i}^{e m}+E_{i}^{\text {had }} \sin \theta_{i}^{\text {had }} \cos \phi_{i}^{\text {had }}\right)
\end{gathered}
$$




$$
\begin{gathered}
p_{y}=\sum_{i=1}^{N_{\text {towers }}}\left(E_{i}^{\text {em }} \sin \theta_{i}^{\text {em }} \sin \phi_{i}^{\text {em }}+E_{i}^{\text {had }} \sin \theta_{i}^{\text {had }} \sin \phi_{i}^{\text {had }}\right) \\
p_{z}=\sum_{i=1}^{N_{\text {towers }}}\left(E_{i}^{\text {em }} \cos \theta_{i}^{\text {em }}+E_{i}^{\text {had }} \cos \theta_{i}^{\text {had }}\right)
\end{gathered}
$$

The direction and transverse energy of the jet are then computed:

$$
\begin{gathered}
\theta=\arctan \left(\frac{\sqrt{p_{x}^{2}+p_{y}^{2}}}{p_{z}}\right) \\
\phi=\arctan \left(\frac{p_{y}}{p_{x}}\right) \\
E_{T}=E \sin \theta
\end{gathered}
$$

and the pseudo-rapidity from (2.2).

\subsubsection{Jet Corrections}

Jets are reconstructed from raw calorimeter energies. The raw energy does not predict the transverse momentum of the parton that induced the jet due to both instrumental and physical reasons. In order to obtain the best estimate for the parton energy, the jet energy is corrected with a standard method developed at CDF [37]. The correction leaves the direction of the jets unchanged. The original parton transverse momentum is expressed in the following equation:

$$
p_{T}^{\text {parton }}=\left(p_{T}^{\text {jet }} \cdot C_{\eta}-C_{M I}\right) \cdot C_{A b s}-C_{U E}+C_{O O C}=p_{T}^{\text {particle }}-C_{U E}+C_{O O C}
$$

The corrections categorized as instrumental are:

- $C_{\eta} ; \eta$-dependent correction applied in order to provide uniform calorimeter response in the entire pseudo-rapidity range. Dependence on $\eta$ is caused by cracks between the calorimeter components found at $\eta=0$ between the two halves of the central calorimeter, and at $|\eta| \approx 1.1$ between the central and plug calorimeters. The method used in this correction implicitly takes into account the transverse spreading of the calorimeter showers outside the jet cone and the $\eta$ dependence of gluon radiation and multiple interactions. 
- $C_{M I}$; as it was mentioned in Section 2.3.6, at high instantaneous luminosity multiple interactions can occur in one bunch crossing. The extra interactions are usually minimum bias events overlapping the jets from the hard scattering. This correction subtracts the extra energy.

- $C_{A b s}$; the absolute correction compensates for the difference between the transverse momentum of a particle and its corresponding jet. After applying this, the jet energy scale is assumed to be detector independent. The correction is derived from Monte Carlo, therefore, it relies on the jet modeling in the simulation. It corrects for the following effects:

1. non-linear calorimeter response to charged and neutral hadrons as function of the momentum,

2. intrinsic energy resolution of the calorimeter,

3. different track reconstruction efficiency in data and simulation,

4. stability of the calorimeter energy scale.

The physical effects are taken into account in the Underlying Event $\left(C_{U E}\right)$ and Out-of-Cone $\left(C_{O O C}\right)$ corrections. The underlying event is due to hadrons that originate from the break-up of the proton and antiproton in the collision and may also contain hadrons from initial state radiation. The out-of-cone correction compensates for parton radiation that falls outside the jet cone.

In this analysis, we only correct jets for the instrumental effects which are common in missing energy based analyses at CDF. In turn, we use a more realistic simulation which contains minimum bias events with the number of extra interactions proportional to the average instantaneous luminosity. The choice of the small cone-size of 0.4 was motivated by the b-jet identification algorithm. This leads to lower dijet masses in the Higgs modeling, but does not affect the dijet mass resolution negatively. Generally, the jet correction raises the energy of the jets by about 10-30\%. 
The $E_{T}$ of the jets are corrected when

- The uncorrected jet $E_{T}>10 \mathrm{GeV}$

- $|\eta|$ of the jet $<2.0$

- The electromagnetic fraction of the total jet energy is greater than 0.1 and less than 0.9

\subsection{Missing Transverse Energy $\left(\notin_{T}\right)$}

The total energy and momentum in every collision is conserved, so is the total momentum projected in the transverse plane, called missing transverse energy $\left(\mathbb{E}_{T}\right)$. $\mathscr{E}_{T}$ is one of the most important variables in this search since the signal events contain neutrinos which escape the detector and produce imbalance in the measured total energy. The missing transverse energy is reconstructed entirely based on calorimeter information. Since the CDF calorimeters do not have perfect $4 \pi$ coverage, the energy and momentum of particles moving in the $z$-direction are not entirely measured. The total momentum and energy in the transverse plane however is well reconstructed. Missing energy therefore should indicate the presence of neutrinos in the event.

\subsubsection{E $_{T}$ Reconstruction}

Missing transverse energy is also computed from raw tower energies. All towers up to $|\eta|<3.6$ are taken into account thus the $\mathbb{E}_{T}$ contains both clustered energy (i.e. energy in jets) and unclustered energy. It is a vector quantity with the following components and magnitude:

$$
\begin{aligned}
& \mathscr{E}_{x}=-\sum_{i=1}^{N_{\text {towers }}} E_{T}^{i} \cos \phi^{i} \\
& \mathscr{E}_{y}=-\sum_{i=1}^{N_{\text {towers }}} E_{T}^{i} \sin \phi^{i}
\end{aligned}
$$


and

$$
\notin_{T}=\sqrt{\mathscr{E}_{x}^{2}+\mathbb{E}_{y}^{2}}
$$

where $E_{T}^{i}$ is the total hadronic and electromagnetic energy in the $i^{\text {th }}$ tower given in (3.2) with a threshold of $100 \mathrm{MeV}$, and

$$
\phi^{i}=\frac{\mathbb{E}_{T}^{e m, i} \phi^{e m, i}+\mathbb{E}_{T}^{h a d, i} \phi^{h a d, i}}{\mathbb{E}_{T}^{e m, i}+\mathbb{E}_{T}^{h a d, i}}
$$

The direction assigned to the $\mathbb{E}_{T}$ is defined by the azimuthal angle

$$
\phi_{\not_{T}}=\arctan \left(\frac{\phi_{y}}{\phi_{x}}\right)
$$

The missing transverse energy is a quantity that describes a whole event rather than a single object, therefore it can rarely be identified with a single neutrino. There are several reasons why $\mathbb{E}_{T}$ is created in an event:

- One or more neutrinos are created as decay products in the $p \bar{p}$ collision. This is what we wish to measure. In some events, such as $W+0$ jet, the missing $E_{T}$ can be associated with a single neutrino originating from the $W$ decay.

- Multiple interactions which are significant at high instantaneous luminosity.

- Charged leptons such as muons escape the event with minimum energy deposition in the calorimeters.

- Cosmic muons traversing the detector promote low $\not_{T}$ to higher value.

- Beam halo-muons crossing a row of calorimeter towers along the $z$-axis deposit energy to the calorimeters asymmetrically in $\phi$.

- Proton - antiproton beam remnants (underlying event) and beam losses at large $|\eta|$ towers.

- High- $p_{T}$ objects that are under measured due to reaching the calorimeters at or near to uninstrumented regions (cracks and the "chimney"). 
- Hot towers in the calorimeter (PMT spikes).

- Jets that are often under measured especially due to neutrinos within the semileptonically decaying heavy flavor jets.

- In the special case of triggers, the tower energy resolution has been observed to play a significant role. At Level 1 the minimum trigger tower energy is $1 \mathrm{GeV}$ which degrades the $\mathbb{E}_{T}$ resolution (see Chapter 4 ).

Beam effects, cosmic muons and some instrumental effects are removed in the very first step of the analysis by discarding problematic events, see Section 3.2.2.

Mismeasurement of jets is partially corrected within the remaining events, see Section 3.2.3. Not all the effects can be treated. Since we do not have corrections specifically for heavy flavor jets, we need to rely on the simulation. We also simulate the topology of events and the multiple interactions. (Note that nearly half of the events have more than one vertex in the entire $1 \mathrm{fb}^{-1}$ data.) Events with high$p_{T}$ muons are rejected in the preselection cuts because signal events do not contain charged leptons; therefore, the $\mathbb{E}_{T}$ is not corrected for muons.

\subsubsection{Quality Selection for Missing $E_{T}$ Analyses}

The $E_{T}$ resolution have been studied by the $\not_{T}$ working group. All $\notin_{T}$ based analyses apply a set of quality cuts on the data ("clean-up cuts") and similar correction methods [38]. Here is a summary of these cuts organized in three passes:

- Pass 1 requirements

- At least one central jet $(|\eta|<0.9)$ with $E_{T}>10 \mathrm{GeV}$,

- Event Electromagnetic Fraction (EEMF):

$$
E E M F=\frac{\sum_{j=1}^{N_{j e t}} E_{T}^{j} \cdot E M F_{j}}{\sum_{j=1}^{N_{j e t}} E_{T}^{j}}>0.1
$$

where $E M F_{j}$ is the fraction of the jet energy deposited in the electromagnetic calorimeter. Only jets with $E_{T}^{\text {Raw }}>10 \mathrm{GeV}$ are considered, 
- At least one COT track with $p_{T}>0.5 \mathrm{GeV} / \mathrm{c}$ and an axial super layer with six or more hits.

- Pass 2 requirements

- Event Charge Fraction (ECHF):

$$
E C H F=\frac{\sum_{j=1}^{N_{j e t}} C H F_{j}}{N_{j e t}}>0.1
$$

where $\mathrm{CHF}_{j}$ is the jet charge fraction defined as the sum of the $p_{T}$ of the tracks matched to the jet over the jet $E_{T}$ :

$$
C H F_{j}=\frac{\sum_{i=1}^{N_{\text {tracks }}} p_{T}^{j i}}{E_{T}^{j}}
$$

- At least one good primary vertex in the event.

- Pass 3 requirements

- The chimney is a hole in the calorimeter at $\phi=\left(60^{\circ}, 100^{\circ}\right)$ and $\eta=$ $(0.5,1.0)$ that hosts cryogenic and instrumental connections to the inner detector. Jets that fall into the chimney region are almost certainly mismeasured, therefore we discard any event that has such a jet with $E_{T}>10 \mathrm{GeV}$.

- Event primary vertex falls within $|z|<60 \mathrm{~cm}$ of the nominal interaction point at the detector center.

- The beam halo energy usually appears in a row of towers at $\phi=0$. It was found that the previous selection criteria are sufficient to eliminate events with beam halo muons, therefore no further treatment is required.

- Total calorimeter energy less than $2 \mathrm{TeV}$.

\subsubsection{Missing Transverse Energy Correction}

Preliminary studies concluded that the best $\mathbb{E}_{T}$ resolution is obtained when using jets corrected up to $C_{A b s}$ in the standard jet corrections (Section 3.1.2). The cone-size 
of the jets is 0.4 in order to provide the best angular resolution. The corrected $\notin_{T}$ is given by the following formula:

$$
\begin{aligned}
& \mathscr{E}_{x}^{\text {Corr }}=\not_{x}^{\text {Raw }}-\sum_{i=1}^{N_{\text {jets }}}\left(E_{x}^{\text {Corr }, i}-E_{x}^{\text {Raw }, i}\right) \\
& \mathscr{E}_{y}^{\text {Corr }}=\not_{y}^{\text {Raw }}-\sum_{i=1}^{N_{\text {jets }}}\left(E_{y}^{\text {Corr }, i}-E_{y}^{\text {Raw }, i}\right)
\end{aligned}
$$

The azimuth of $\notin_{T}$ in the transverse plane is calculated as:

$$
\varphi_{\not_{T}}=\arctan \left(\frac{\not_{y}^{C o r r}}{\not_{x}^{C o r r}}\right)
$$

\subsection{Track Reconstruction}

The tracking system of the CDF detector is briefly introduced in Section 2.3.1. It detects and reconstructs trajectories of charged particles. This information is used in primary vertex reconstruction, lepton identification, and heavy flavor tagging. Electron identification combines tracking and calorimeter information. Muon and one-prong tau identification is entirely based on tracking.

The tracking detectors are enclosed by the CDF solenoid, which provides a uniform magnetic field inside the detector with field lines parallel to the $z$-axis. Electrically charged particles traveling through magnetic field experience a Lorentz force that is perpendicular to the field lines and in strength proportional to the speed of the particle as well as its charge. The resulting trajectory is a helix. At CDF the following parameters are used in the description of the trajectory in three dimensions:

- $C$, half-curvature of the trajectory defined as

$$
C=\frac{1}{2 Q \rho}
$$

where $\rho$ is the radius of the projection of the trajectory to the transverse plane. It carries the same sign as the charge of the particle and is inversely proportional to the $p_{T}$ of the track. 
- $\cot \theta_{0}$, cotangent of the polar angle at the trajectory point of closest approach to the origin.

- $d_{0}$, the impact parameter, is the minimal distance between the trajectory and the origin in the transverse plane

$$
d_{0}=Q \cdot\left(\sqrt{x_{0}^{2}+y_{0}^{2}}-\rho\right)
$$

where $x_{0}$ and $y_{0}$ are the coordinates of the center of the helix circle in the transverse plane.

- $\phi_{0}$, the azimuth of the trajectory at the point of closest approach to the origin.

- $z_{0}$, the $z$ position of the trajectory point closest to the origin

Two tracking algorithms are implemented at CDF. In the central region within the $|\eta|<1$ coverage of the COT, a more robust search algorithm is used called "OutsideIn". Outside the central region, the tracking is based on the "Silicon Stand-Alone" tracking.

The track reconstruction starts in the COT, where the hits are better separated and their multiplicity is lower than in the silicon detectors. The algorithm reconstructs track segments with 4 or more hits in each super layer. It then tries two methods to assemble the segments of a track. First it searches for segments that fit on a common circle. If that fails, a circular fit of the segments is created constrained to intersect the beamline, and consistent hits from other super layers are added to the reference track. The COT tracking efficiency is nearly $100 \%$ for isolated tracks with $p_{T}>5 \mathrm{GeV} / \mathrm{c}$.

The "Outside-In" tracking extends the COT tracks into the silicon. It attaches axial silicon hits to COT tracks and then performs pattern recognition on the smallangle and $90^{\circ}$ stereo strips. The information provided by the external seed reduces the number of possible track combinations from a quadratic to a linear function of the hit number. At each wafer intersected by the seed track, every hit is attached 
to a separate copy of the seed track. Then the track is extrapolated to the next wafer where the same matching procedure is performed until the track reaches the innermost silicon wafer. At the end, the track combination with the highest number of hits and lowest $\chi^{2} /$ dof is kept.

The remaining hits in the silicon that are not associated with COT seed tracks are used to construct the "Silicon Stand-Alone" tracks which are not used in this analysis.

\subsection{Primary Vertex Reconstruction}

Vertex location is required to calculate transverse energies in the calorimeter towers and derived objects such as jets and missing energy, since the angle $\theta$ is defined by the vector pointing from the vertex to the shower maximum detectors within the calorimeter towers (see Equation 3.2). Using the detector center instead of the $z$ position of the vertex in the $\mathbb{E}_{T}$ calculation has an impact on the $\mathbb{E}_{T}$ beyond the level of the corrections derived from jets (i.e. this is the main difference between the online L3 and offline $\mathbb{E}_{T}$ ). Z-vertex locations have a Gaussian spread of $\sigma$ about $30 \mathrm{~cm}$ around the detector center in data events.

Vertices are found in the intersection of multiple tracks [39]. Their quality is quantified by the number of COT tracks used in the reconstruction. The $z$-coordinate of the vertex is calculated by

$$
z_{v t x}=\frac{\sum_{i=1}^{N_{\text {tracks }}} z_{i}^{0} / \sigma_{i}^{2}}{\sum_{i=1}^{N_{\text {tracks }}} 1 / \sigma_{i}^{2}}
$$

where $z_{i}^{0} \pm \sigma^{i}$ is the $z$-position of the $i^{\text {th }}$ track (see Section 3.3). Good vertices are ordered by the total scalar sum of the track $p_{T}$-S. The one with the highest $p_{T}$-sum is considered the primary vertex.

The primary vertex location serves as the point of reference for finding secondary (displaced) vertices. 


\subsection{Secondary Vertex and B-Tagging}

Heavy flavor jets are identified with three different algorithms at CDF:

- The Soft Lepton Tagger finds jets with semi-leptonically decaying heavy flavor (cbt) quarks [40]. These jets contain non-isolated soft leptons (electron or muon) originating from the $W^{ \pm}$bosons that are emitted in weak decays of heavy quarks

- The Jet Probability algorithm takes advantage of the lifetime of heavy flavor hadrons [41]. Tracks in heavy flavor jets are displaced. The jets are assigned a probability of originating from the primary interaction point based on their associated tracks.

- The Secondary Vertex Tagging algorithm (SecVtx [39]) is built on the same characteristics of heavy flavor jets, but is optimized for b-jet identification.

Our search uses the SecVtx algorithm.

B-quarks that are produced in the hard scattering undergo fragmentation and hadronization and form B-hadrons. B-hadrons have a fairly long lifetime, on average $c \tau=450 \mu \mathrm{m}$ which translates to a few millimeters of path length in the detector's reference frame. These hadrons travel away from the primary vertex and subsequently decay to hadrons through a cascade of particles. The charged decay products are often reconstructed as displaced tracks. The intersections of these tracks form secondary vertices at the points where the B-hadrons decay.

The SecVtx algorithm searches for displaced secondary vertices by combining tracks within "taggable" jets (Figure 3.1). Jets are taggable if $E_{T}^{\text {Raw }}>10 \mathrm{GeV}$, $|\eta|<2.4$, and have at least two good tracks. It first combines three or more tracks with looser selection requirements. If that fails, pairs of tracks that pass tighter quality requirements are tested. The displacement of the secondary vertex with respect to the primary vertex in the transverse plane is given by

$$
L_{x y}=\vec{d} \cdot \hat{p_{T}}
$$




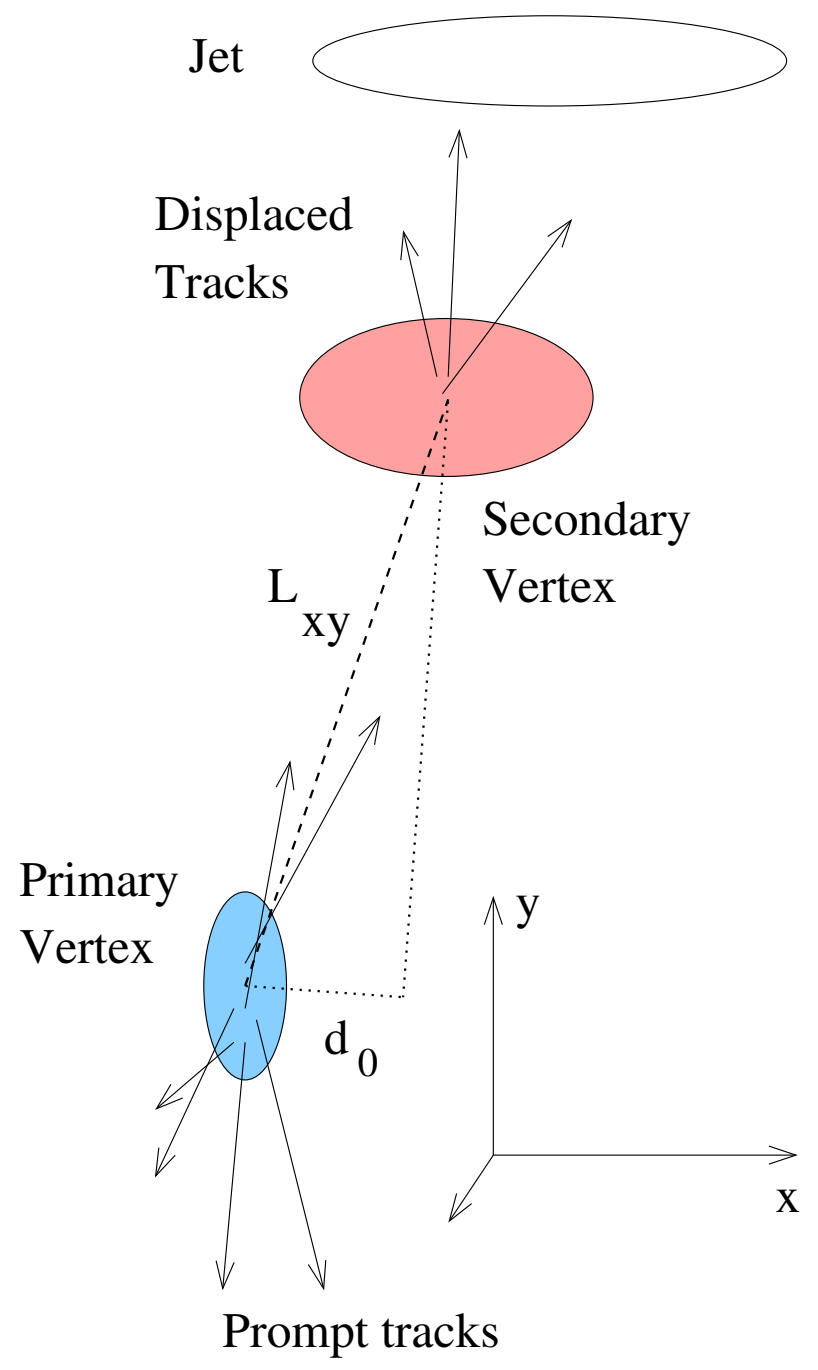

Fig. 3.1. Schematic diagram of the secondary vertex tagging [42]

where $\vec{d}$ is the displacement of the secondary vertex and $\hat{p_{T}}$ is the unit vector of the jet momentum. Thus $L_{x y}$ is positive if the displacement points along the jet momentum, and negative if it points to the opposite direction.

Jets are tagged positively if $L_{x y} / \sigma_{L_{x y}}>3$ and negatively if $L_{x y} / \sigma_{L_{x y}}<-3$. Jets with negative tags cannot be real heavy flavor jets, because the B-decay products would move to a direction opposite to the jet. Negative tags are due to resolution effects in the tagging, and are usually high- $p_{T}$ light flavor (uds) jets. Similar phenom- 


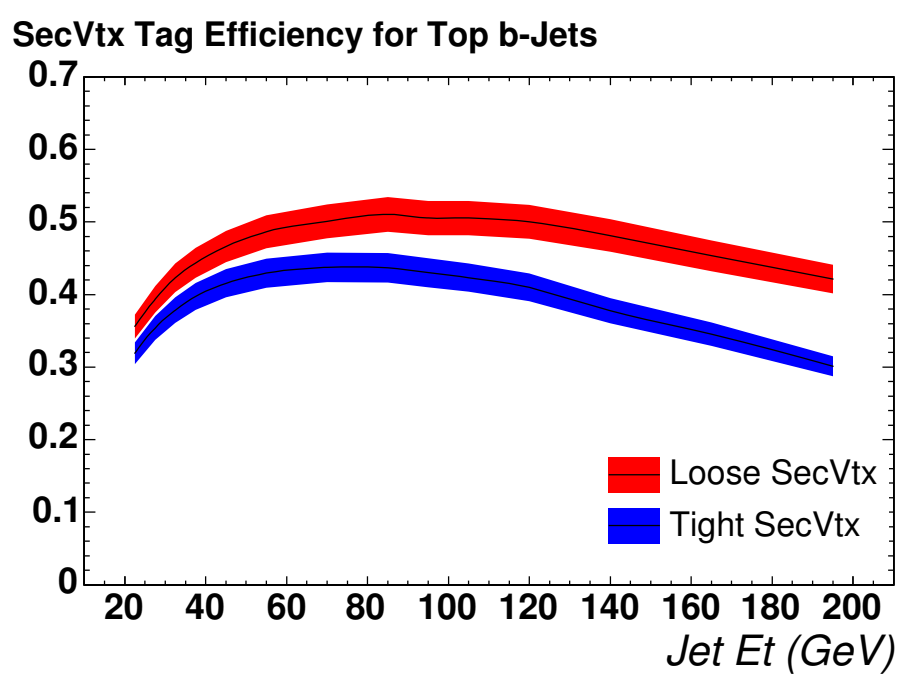

Fig. 3.2. Tagging efficiency of the tight and loose SecVtx algorithm as function of the tagged jet $E_{T}[42]$

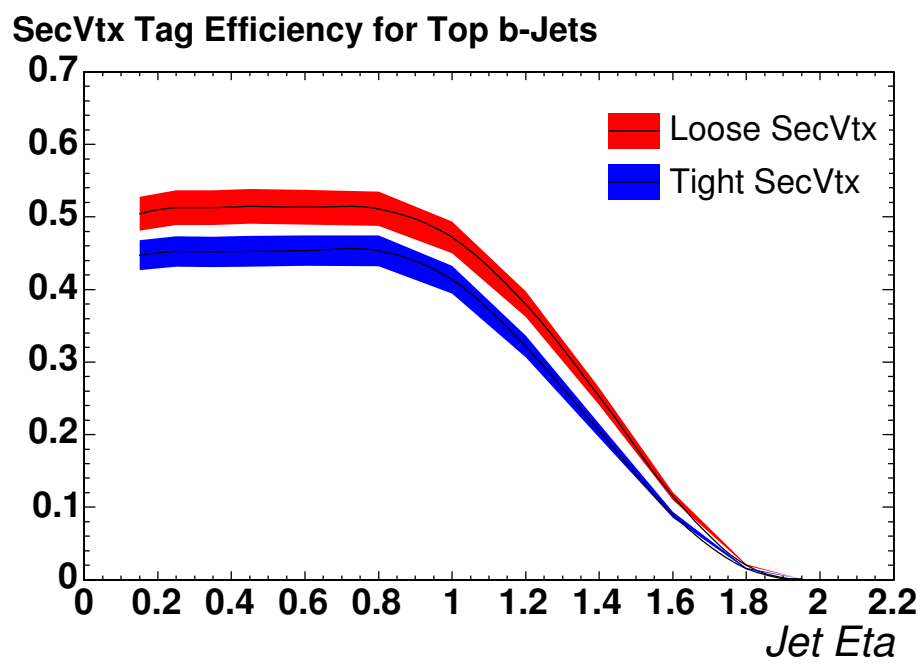

Fig. 3.3. Tagging efficiency of the tight and loose SecVtx algorithm as function of the tagged jet $\eta[42]$

ena are observed in the simulation of positive tags. The positively tagged light flavor jets are called mistags. 
There are two settings for SecVtx, one with looser and one with tighter track requirements. The loose tagger has higher efficiency for b-jets than the tight, but it also suffers a higher mistag rate. In this analysis, we use the tight setting. The efficiency for the two settings are shown in Figure 3.2 and 3.3 as functions of the jet $E_{T}$ and $\eta$. A degradation of track reconstruction is observed at $|\eta|>1.1$ outside the COT coverage. The efficiency is defined on taggable jets.

The detector simulation is reported to overestimate the tracking resolution. As a consequence, the tagging efficiency is higher in Monte Carlo than in data. We apply a weighting factor to Monte Carlo events to compensate for this effect. Currently the tagging Scale Factor (SF) for the tight tagger is 0.95 $\pm 0.04[42,43]$.

\subsection{Lepton Identification}

Although, no leptons are expected in the signal events, lepton identification is needed for vetoing high- $p_{T}$ lepton background. It is also important to ensure that signal events produced in the $W^{ \pm}$associated channel which are analyzed in a dedicated $W H$ search are not double-counted here. Our goal is to reject all electrons reconstructed in the calorimeters and muons identified as isolated high- $p_{T}$ tracks.

\subsubsection{Electron Reconstruction}

Electrons are measured in the Electromagnetic Calorimeters (see Section 2.3.3). Incident electrons induce showers across multiple calorimeter towers. The energy of the showers appears in clusters in the $\phi-\eta$ coordinate system. The clustering algorithm looks for EM-objects in the CEM and PEM separately [44]. It starts by creating an $E_{T}$-ordered list of possible seed towers that are in the fiducial region and have $E_{T}^{e m}>2 \mathrm{GeV}$. Then towers within the fiducial regions (including seeds) adjacent to the available highest $E_{T}$ seed are checked. They may belong to the cluster if they are in the same detector (CEM or PEM) as the seed, have not been already used, and have $E_{T}>0 \mathrm{MeV}$ for CEM or $E_{T}>100 \mathrm{MeV}$ for PEM. Clusters in CEM can grow 
only in $\eta$ by \pm 1 physical tower from the seed. In the PEM, the maximal cluster size is $2 \times 2$ including the seed. A cluster is found if the total EM-energy passes $E_{T}^{e m}>2 \mathrm{GeV}$ (default), and $E_{T}^{h a d} / E_{T}^{e m}<0.125$ where $E_{T}^{\text {had }}$ is the hadronic energy within the seed tower in CEM and in the whole cluster in PEM. If the total energy of the cluster is greater than $100 \mathrm{GeV}$ then the cluster is always kept.

After all clusters are found, tracks from the default collection are matched with them computing the cluster center with the energy weighted average of the CES/PES coordinates of the cluster towers. The central electron candidates must have a matching COT track.

In this analysis, we apply additional cuts listed in Table 3.1 for discriminating electrons with at least $10 \mathrm{GeV}$ transverse energy from electron faking objects such as photons, isolated charged hadrons, and jets. The cuts are different in the central and plug regions. The following variables are used:

- $E_{T}$, total transverse energy,

- $E / P$, the ratio between the cluster energy to the momentum of the associated track,

- $E^{\text {had }} / E^{e m}$, ratio of the total hadronic cluster energy to the total EM energy,

- $L_{s h r}$, comparison of the cluster's lateral shower profile with the test beam electrons,

- $|\Delta X|$ and $|\Delta Z|$, the distances in the transverse plane and in the $z$-direction between the cluster position from the CES measurements and the extrapolated track associated with the cluster,

- Iso4, the ratio of the transverse energy surplus in a cone of radius $R=0.4$ around the cluster center to the transverse energy of the cluster,

- $\chi^{2}$, comparison of the CES shower profile with the test beam electrons, 
- $\chi^{2}(3 \times 3)$, comparison of the PEM shower profile to that of test beam electrons. (The test beam results were obtained using $3 \times 3$ cluster size.)

These cuts have been chosen such that events passing any combinations of selection thresholds commonly used in electron-based searches are contained. The ratio of selection efficiencies between simulation and data are consistent with 1.0 within a few per cent uncertainty [45].

Table 3.1

Summary of electron identification cuts.

\begin{tabular}{|lr|}
\hline \multicolumn{2}{|c|}{ Central Electrons } \\
\hline$E_{T}$ & $\geq 10 \mathrm{GeV}$ \\
$E / P$ & $<4$ \\
$E^{\text {had }} / E^{e m}$ & $<0.125$ \\
$L_{\text {shr }}$ & $<0.2$ \\
$|\Delta X|$ & $<3 \mathrm{~cm}$ \\
$|\Delta Z|$ & $<5 \mathrm{~cm}$ \\
$I s o 4$ & $<0.2$ \\
$\chi_{\text {strip }}^{2}$ & $<10$ \\
\hline \hline \multicolumn{2}{|c|}{ Plug Electrons } \\
\hline$E_{T}$ & $\geq 10 \mathrm{GeV}$ \\
$E^{\text {had }} / E^{\text {em }}$ & $<0.125$ \\
$\chi^{2}(3 \times 3)$ & $<10$ \\
\hline
\end{tabular}

\subsubsection{Isolated Tracks}

Muons are detected by the muon-system placed in the outermost layer of the CDF detector because of the highly penetrating nature of muons. Hits in the muon detectors are linked together to form track segments called stubs. These track segments 
are matched to extrapolated COT tracks with at least $p_{T}>10 \mathrm{GeV}$ and energy deposition in the calorimeter that is consistent with minimum ionizing particles. Isolated tracks that do not have associated stubs are also considered muon candidates (called stubless muons). The initial goal of this analysis was to search for Higgs events in the $Z H \rightarrow \nu \bar{\nu} b \bar{b}$ channel where no isolated charged leptons therefore no isolated tracks are expected. Since all muons reconstructed at CDF have associated isolated tracks, it makes sense to loosen up the muon selection to the level of stubless muons and establish a separate analysis for low-purity leptons. An isolated track veto will also reject events with hadronically decaying high- $p_{T}$ tau leptons.

Table 3.2

Summary of isolated track identification cuts.

\begin{tabular}{|lr|}
\hline \multicolumn{2}{|c|}{ Isolated Tracks } \\
\hline$p_{T}$ & $\geq 10 \mathrm{GeV}$ \\
$E^{e m}$ & $>0$ and $<2 \mathrm{GeV}$ \\
$E^{\text {had }}$ & $>0$ and $<6 \mathrm{GeV}$ \\
$\left|Z_{0}\right|$ & $<60 \mathrm{~cm}$ \\
$I s O 4 / p_{T}$ & $<0.1$ \\
$N_{C O T}^{A x}(\geq 5)$ & $\geq 3$ \\
$N_{C O T}^{S t}(\geq 5)$ & $\geq 2$ \\
\hline
\end{tabular}

The isolated track identification cuts are listed in Table 3.2 and contain the following variables:

- $p_{T}$, the transverse momentum of the isolated track,

- $E^{e m}$ and $E^{h a d}$, the EM and hadronic energy deposits in the calorimeter associated with the isolated track,

- $\left|Z_{0}\right|$, the $z$-coordinate of the track at the point of closest approach to the detector origin, 
- $I s o 4 / p_{T}$, the relative track isolation,

- $N_{C O T}^{A x}(\geq 5)$ and $N_{C O T}^{S t}(\geq 5)$, the number of axial and stereo COT layers that have at least 5 hits.

\subsection{Quality Assurance and Run Selection}

Tevatron stores are split into runs in CDF because their duration is too long for continuous data-taking. The storage unit for book keeping data within runs is called runsection. The duration of one runsection is about 0.1-0.5 minutes depending on the instantaneous luminosity. Every runsection has a unique identifier number in the SQL database which can be used to query various runsection properties such as time-stamp, integrated luminosity, first and last event number, etc.

The Data Quality Monitor (DQM) system continuously monitors the detector components during data-taking. Every subdetector has a quality bit in the database that is set if the detector was functional during an entire run or runsection period. The first step of the analysis is to compile a list of good runs (runsections ranges) requiring that all subdetectors relevant to the analysis have the quality bit set. The list contains runs with good calorimeters and tracking system since these detectors are used for detecting jets, tagging heavy flavor jets, and for lepton identification. Only runsections in the "good run list" are processed in the analysis. The amount of the analyzed data is calculated as the sum of the integrated luminosities in the runsections. We use $0.973 \mathrm{fb}^{-1}$ of data collected by the MET35_\&_TWO_JETS trigger (see Chapter 4). 


\section{TRIGGER EFFICIENCY}

At hadronic colliders such as the Tevatron, only a small fraction of bunch crossings produce an actual hard scattering with considerable momentum transfer. These events have to be selected in real-time when about 1.7 million (non-empty) bunch crossings happen every second. The selection is done by a fast decision making acquisition system, called the trigger system. A short introduction to the structure of the triggers was given in Section 2.3.7.

The problem in fact is more complex than just selecting events with minimal momentum transfer between the outgoing particles. The frequency of events with considerable transverse energy is far larger than the rate at which the entire trigger system can process them; therefore, the triggers have to be designed around specific physics goals. Since the initial instantaneous luminosity in the Tevatron stores keeps increasing, this represents a continuous work on both monitoring old and designing new smarter trigger configurations.

Because the physics analysis starts at the online level, one has to ensure that the event selection is efficient enough for discovering new physics, and the trigger efficiency is well understood. It is no surprise, therefore, that a large fraction of the analysis work lies in trigger efficiency study.

When this Higgs search started in Run II, about $300 p^{-1}$ data had been accumulated with already existing triggers. Thus the previous question was reversed, and we needed to find the best trigger for the analysis.

\subsection{The Missing Transverse Energy Trigger}

Triggers are grouped around underlying physics goals. The data collected by a group of triggers is called a dataset. The data is processed in streams denoted by 
single letters. The trigger paths focusing on $\mathbb{E}_{T}$ are in the dataset called "emetXX" where the initial "e" refers to the stream and "XX" is the hexadecimal serial number of the processing cycle. A list of the datasets and the triggers in the $\mathbb{E}_{T}$ can be found in Appendix B.

We chose the trigger called MET35_\&_TWO_JETS from the $\mathbb{E}_{T}$ dataset which was later changed to MET35_\&_CJET_\&_JET. The first run collected by the MET35_\& _TWO_JETS trigger was taken on the $22^{\text {nd }}$ of July, 2002. Its definition changed on March 27, 2005 to require one central jet $(|\eta|<1.1)$. This modification did not cause any signal efficiency loss, because a central jet is implicitly required through the b-tag selection and there is an explicit central jet selection among the $\mathbb{E}_{T}$ quality cuts. The paths are defined with the following selections:

MET35_\&_TWO_JETS :

- L1 : $\operatorname{Raw} \not_{T}>25 \mathrm{GeV}$

- L2 : TWO JETS $E_{T}>10 \mathrm{GeV}$

- L3 : Raw $\notin_{T}>35 \mathrm{GeV}$

MET35_\&_CJET_\&_JET :

- L1 : $\operatorname{Raw} \notin_{T}>25 \mathrm{GeV}$

- L2 : JET $E_{T}>10 \mathrm{GeV}$ and CJET $E_{T}>10 \mathrm{GeV}|\eta|<1.1$

- L3 : Raw $\mathbb{E}_{T}>35 \mathrm{GeV}$

\subsubsection{Level $1 \not_{T}$ Reconstruction}

The Level 1 calorimeter trigger system consists of custom electronics designed for fast decision making. It reconstructs objects such as electrons, photons and jets, and event variables such as missing transverse energy and total transverse energy. Physical towers are organized in a $24 \times 24$ array of logical trigger towers in the $\phi-\eta$ plane where one tower generally corresponds to a $15^{\circ}$ by 0.2 spatial angle and stores 
the tower transverse energy on 8 bits in units of $0.5 \mathrm{GeV}$. The transverse projection of the tower energies are done with the primary event vertex assumed to be at $z=0$. These trigger towers are then paired in $\eta$ reducing the size of the array to $24 \times 12$ and increasing the energy unit of the least significant bit to $1 \mathrm{GeV}$. Jets at Level 1 are identified as trigger tower pairs with energy multiples of $1 \mathrm{GeV}$. The missing and total transverse energies are calculated as vectorial and scalar sums of trigger tower pairs. Consequently, the missing transverse energy has very limited resolution with respect to the offline corrected $\not_{T}$ and is underestimated on average. The threshold is chosen to be as low as possible in order to maximize efficiency but high enough to keep the Level 1 acceptance rate under control.

\subsubsection{Level 2 Jet Trigger}

The $\not_{T}$ and $\sum E_{T}$ values are then carried up to Level 2 where these quantities are not recomputed. In our path, two clusters are selected, which represent two jets. The cluster finding algorithm searches for contiguous group of towers of at least $1 \mathrm{GeV}$ total energy starting from a seed of at least $3 \mathrm{GeV}$. There is no size limit to the clusters. This makes the algorithm vulnerable to high occupancy events. High occupancy can lead to losses in both efficiency and purity. The two-jet trigger discards high- $p_{T}$ dijet events at the per cent level due to merging jets into one big cluster because of connecting small energy towers. At the same time, in high occupancy events low- $p_{T}$ clusters can be promoted to high- $p_{T}$ due to large cluster sizes.

The latter effect is enhanced in the $\mathbb{E}_{T}$ triggers at low jet-selection thresholds. Since only events with at least $25 \mathrm{GeV}$ total energy pass Level 1, there should be at least one object (jet) over this threshold; therefore, one of the $10 \mathrm{GeV}$ cluster requirements is often automatically satisfied by the leading cluster. The frequency distribution of the second leading cluster is an exponentially decreasing function of the cluster energy. Its steep fall makes the number of events passing the trigger 
susceptible to little changes in the cluster energy. The result is a large increase in the trigger cross-section at high instantaneous luminosity.

Correlations between the jet and $\notin_{T}$ selection complicate the determination of the trigger efficiency.

\subsubsection{Level $3 \not_{T}$ Reconstruction}

At Level 3, the full precision of the entire calorimeter is available. The $\not_{T}$ resolution is greatly improved with respect to Level 1 , therefore it is possible to set a higher threshold in the path. The Level 3 threshold is chosen so that the trigger becomes $100 \%$ efficient at about the same offline $\mathbb{E}_{T}$ as the Level 1 selection. There is an enormous event reduction (about two orders of magnitude) in the Level 3 decision due to the exponential nature of the frequency distribution of $\mathbb{E}_{T}$.

\subsection{Computing the Trigger Efficiencies}

There are two strategies that can be followed to study the trigger efficiency. One applies the simulation of the trigger to Monte Carlo events. The other uses data to extract the efficiency.

The first method assumes that every aspect of the collision and the detector response is simulated accurately. The main challenge however is represented by effects that are not always well simulated such as correlations between trigger level variables. Monte Carlo variables would need to undergo a data-to-simulation reweighting to match the data. Therefore, trigger-related uncertainties that are due to instrumental effects and uncertainties due to inaccurate simulation would be entangled.

The second method provides a trigger efficiency that can be used to emulate the effects of the trigger by reweighting the simulated events depending on offline corrected variables that are compared and validated against data. The trigger related uncertainties can be checked independently of the simulation and differences between data and Monte Carlo can be attributed to the physics simulation. The disadvantage 
of this method is that it needs large unbiased data samples. Since the physics content of data events is generally not known, the offline analysis cuts have to be reproduced on the unbiased data samples as closely as possible. At the same time, the less biased a dataset is the smaller fraction of it passes the offline analysis cuts representing a statistical limitation to this method. The solution is to dissect the trigger path into components and check them individually on relatively unbiased datasets. Such datasets are collected by back-up triggers (see Appendix B).

We chose the second method for this analysis. The method and the conclusions might not be completely transferable to other analyses using the same trigger. We start by applying a set of requirements which defines events in the region of analysis interest:

- Good run list requiring good calorimetry and good silicon detector during data acquisition (see Section 3.7).

- $\notin_{T}$ clean-up cuts (see Section 3.2.2) and explicit central jet requirement using offline corrected jets: $E_{T}>25 \mathrm{GeV}$ and $|\eta|<0.9$.

- Two jets with $E_{T}>25 \mathrm{GeV}$ and $|\eta|<2.0$ with at least one of them having $E_{T}>35 \mathrm{GeV}$ in order to make the two Level 2 cluster requirement fully efficient. (This selection is modified when studying the jet-related cuts in the trigger.)

- $\Delta R($ jet 1 ,jet 2$)>1.0$ to remove cluster merging (except when plotting this variable, see explanation below.)

- No additional jets having $E_{T}>20 \mathrm{GeV}$ and $|\eta|<2.4$ is allowed.

- $\mathbb{E}_{T}>50 \mathrm{GeV}$ (except when this variable is under study).

The data samples from which the efficiencies are obtained are described in the following subsections. The level-X trigger efficiency is calculated by:

$$
\epsilon_{L X}=\frac{\text { Events passing: preselections, online selections up to LX }}{\text { Events passing: preselections, online selections up to } \mathrm{L}(\mathrm{X}-1) \text { if } \mathrm{X}>1}
$$

where $\mathrm{X}=1,2,3$. Thus the efficiency of the entire path is the product $\epsilon_{L 1} \cdot \epsilon_{L 2} \cdot \epsilon_{L 3}$. 


\subsubsection{Level 1 Trigger Efficiency (L1_MET25)}

The trigger path requires the Level $1 \mathscr{E}_{T}$ to be at least $25 \mathrm{GeV}$. The efficiency of the selection needs to be parameterized with respect to the offline corrected $\mathbb{E}_{T}$, because that is the variable we compare between data and simulation. The simulated events are reweighted by the efficiency corresponding to their offline corrected $\not_{T}$. The turn-on curve is very broad due to the poor resolution at Level 1. After choosing a fairly low Level 1 threshold, however, the trigger becomes fully efficient at large offline $\not_{T}$ values.

In addition to the resolution effects, a hardware feature in the Level 1 calculation makes the trigger discard a small fraction of events with large $\mathscr{H}_{T}$. This problem arises when the energy deposited into a trigger tower at Level 1 exceeds $127 \mathrm{GeV}$ (overflow of the 8-bit variable). In such cases only $127 \mathrm{GeV}$ is used in the $\mathscr{E}_{T}$ calculation. Events containing saturated towers should be automatically accepted by maxing out the $\mathbb{E}_{T}$ value, but it does not happen. The efficiency loss occurs most of the time when the $\mathscr{E}_{T}$ is due to mismeasurement of balancing jets. In such cases, the $\mathscr{\phi}_{T}$ is aligned with either the first or the second jet. In order to eliminate these events, we require the $\mathbb{E}_{T}$ not to be aligned with any of the leading jets in the signal region. (The signal region is actually defined by one of these cuts, see Chapter 6.) This requirement is also commonly used in $\mathscr{H}_{T}$-based searches to reduce the QCD multi-jet background. The trigger efficiency is studied in events with $\Delta \phi\left(\mathbb{E}_{T}, 2^{\text {nd }} \mathrm{jet}\right)<0.4$ where the tower saturation effect is enhanced. The efficiency loss in the this region is $\sim 4 \%$ percent as the fit in the last plot of Figure 4.3 indicates. No loss of efficiency is observed in the second region (Figure 4.2) on the high $\mathscr{H}_{T}$ plateau.

The Level 1 trigger efficiency is computed from a high- $p_{T}$ muon (path CMUP18) and a medium- $p_{T}$ inclusive jet (JET20) data sample. A high- $p_{T}$ inclusive jet sample (JET50) is used as a cross-check with improved statistics at large $\not_{T}$. Results are shown in Figure 4.1. 

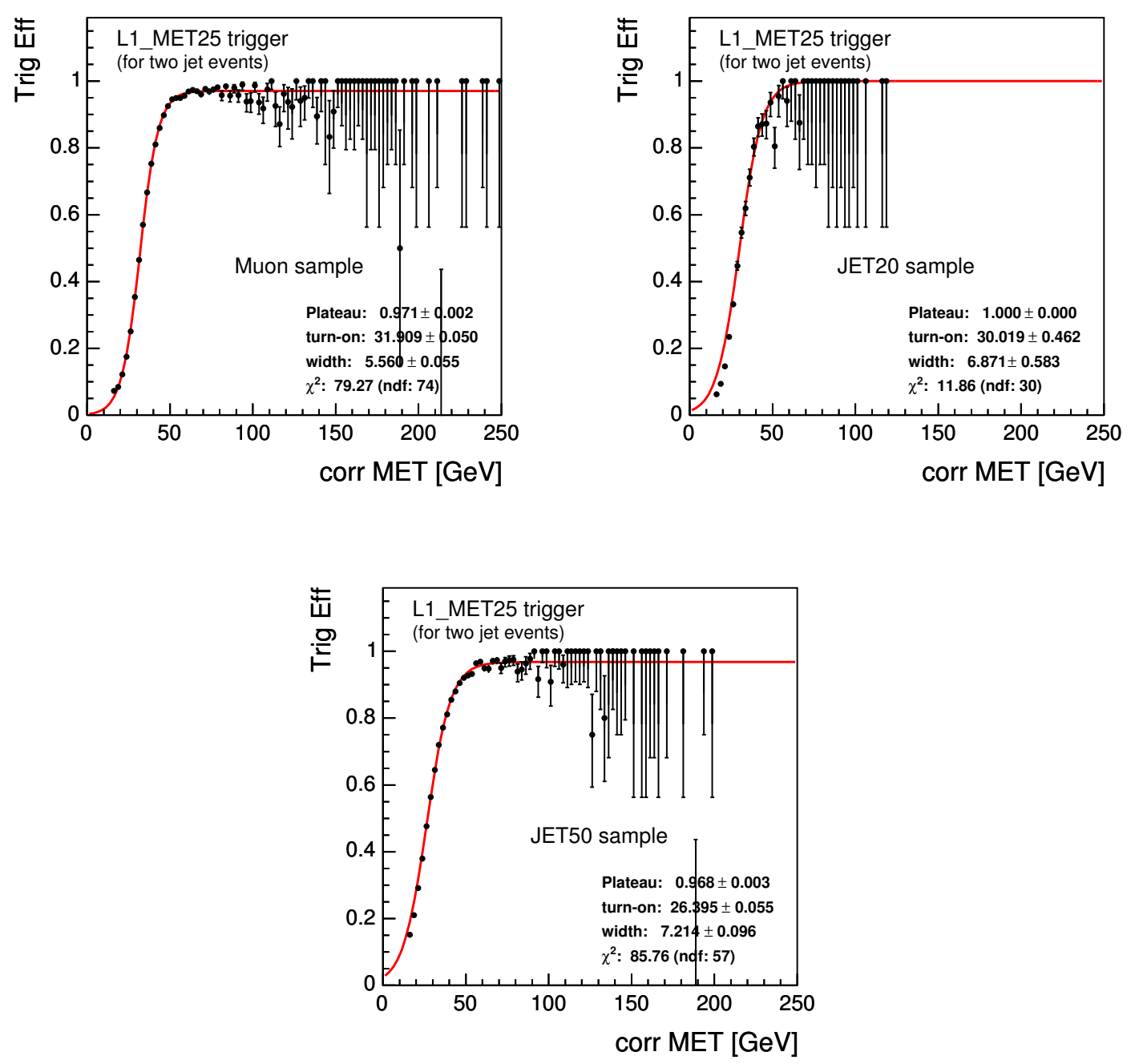

Fig. 4.1. Efficiency of the L1_MET25 trigger in $1 \mathrm{fb}^{-1}$ CMUP18, JET20, and JET50 data samples as function of the corrected $\notin_{T}$. 
Different samples provide slightly different turn-on shapes. The fit is dominated by the large statistics at low $\mathbb{E}_{T}$, thus the curves do not plateau at $100 \%$ efficiency in the JET20 and CMUP18 samples, but the data points indicate full efficiency at high $\notin_{T}$. In order to discard events with fake $\not_{T}$ they are required to pass $\Delta \phi\left(\right.$ jet $\left.2, \mathbb{E}_{T}\right) \geq 0.4$. Results in the three samples are shown in Figure 4.2. The jet samples suffer from small statistics, since these events are initially dominated by dijet production with fake $\mathscr{E}_{T}$. The muon sample that is richer in events with real $\mathbb{E}_{T}$ (and is the most similar to our signal) however has sufficient statistics to determine the efficiency. The $\Delta \phi$ cut is reversed in order to obtain the turn on efficiency in the fake $\mathbb{E}_{T}$ dominated region. Results are presented in Figure 4.3.

The values of the efficiencies determined in the JET50 and the CMUP18 samples are considered nominal in regions $\Delta \phi\left(\right.$ jet $\left.2, \notin_{T}\right)<0.4$ and $\Delta \phi\left(\right.$ jet $\left.2, \notin_{T}\right) \geq 0.4$ respectively.

The following formulae summarize the efficiencies and the systematic uncertainties in the two regions:

- When $\Delta \phi\left(\right.$ jet $\left.2, \notin_{T}\right)<0.4$ :

$$
\epsilon_{L 1}\left(\notin_{T}\right)=\frac{0.97}{1+\exp \left(\frac{26-\not \phi_{T}}{7.4}\right)} ;
$$

where $26 \mathrm{GeV}$ is the turn-on point, and $7.4 \mathrm{GeV}$ is the width (both determined from the fit of JET50 in Figure 4.3). The relative uncertainty on the efficiency:

$$
\frac{\Delta \epsilon}{\epsilon}\left(\notin_{T}\right)= \begin{cases}0.03+0.04 *\left(45-\notin_{T}\right) & \text { if } \notin_{T}<45 \mathrm{GeV}, \\ 0.03 & \text { if } \notin_{T} \geq 45 \mathrm{GeV} .\end{cases}
$$

- When $\Delta \phi\left(\right.$ jet $\left.2, \notin_{T}\right) \geq 0.4$ :

$$
\epsilon_{L 1}\left(\mathscr{H}_{T}\right)=\frac{1}{1+\exp \left(\frac{33-\not \phi_{T}}{6.1}\right)} ;
$$



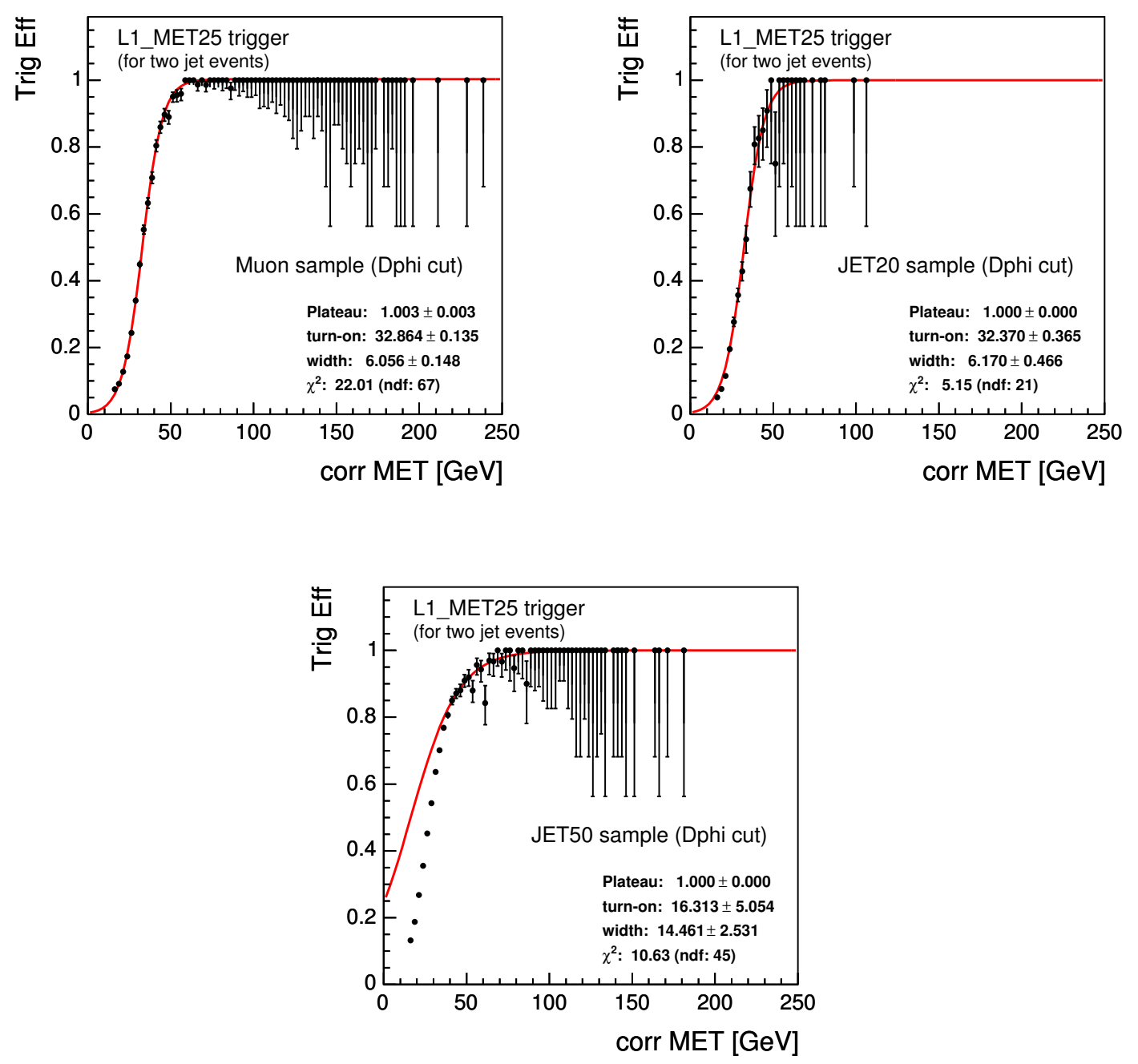

Fig. 4.2. Efficiency of the L1_MET25 trigger in the $1 \mathrm{fb}^{-1}$ CMUP18, JET20 and JET50 data samples as function of the corrected $\not_{T}$ after requiring that $\Delta \phi\left(\right.$ jet $\left.2, \notin_{T}\right) \geq 0.4$. 

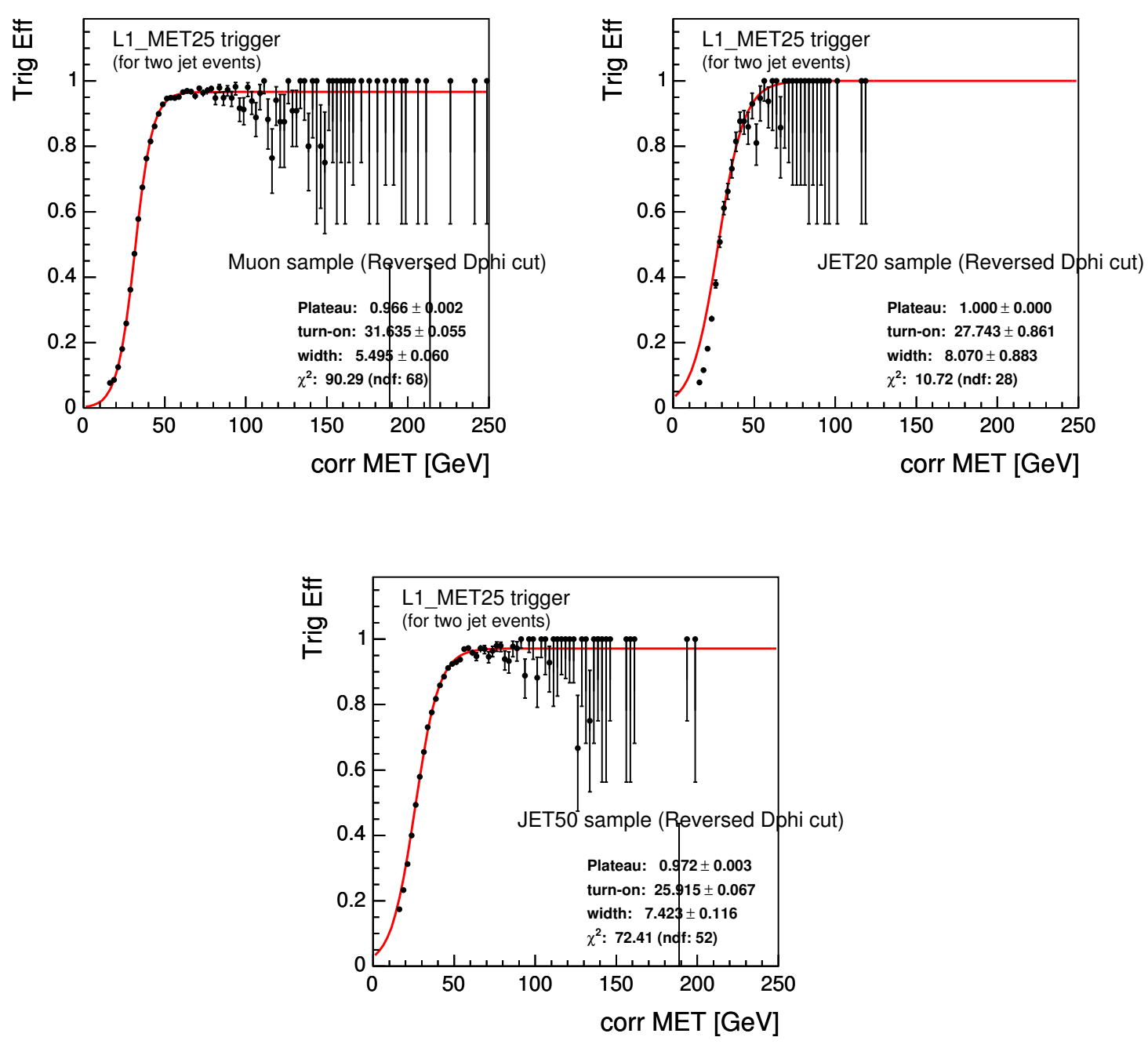

Fig. 4.3. Efficiency of the L1_MET25 trigger in the $1 \mathrm{fb}^{-1}$ CMUP18, JET20 and JET50 data samples as function of the corrected $\not_{T}$ after requiring that $\Delta \phi\left(\right.$ jet $\left.2, \notin_{T}\right)<0.4$. 


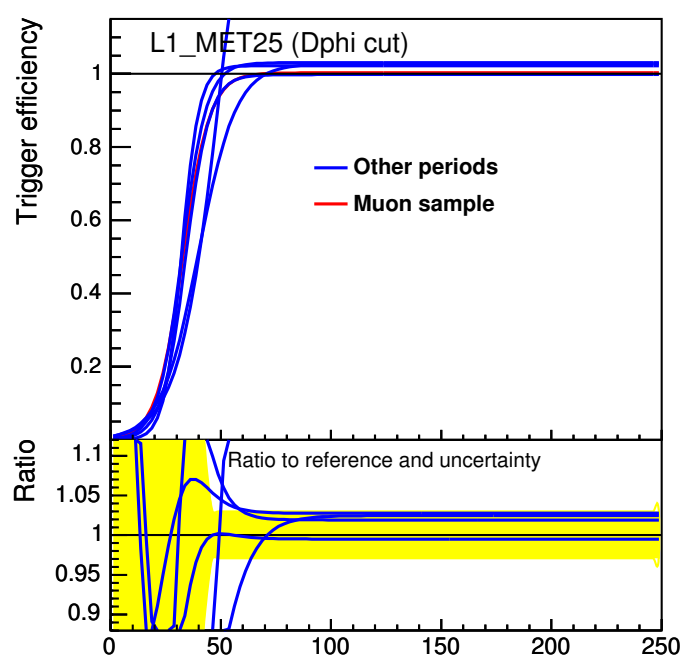

Fig. 4.4. Efficiency of the L1_MET25 trigger in the various run periods in the CMUP18 sample as function of the corrected $\mathbb{E}_{T}$ after $\Delta \phi\left(\right.$ jet $\left.2, \notin_{T}\right) \geq 0.4$ requirement. The lower plot shows the relative difference from the nominal values. The band indicates the relative uncertainty.

and the relative uncertainty is the same as in the first case.

The uncertainties cover the differences between efficiencies computed in the three samples and the differences observed between the fitted curves and the data points.

We have also studied the efficiency as a function of time. Figure 4.4 shows the efficiency in various run-periods in the signal like region $\Delta \phi\left(\right.$ jet $\left.2, \mathscr{E}_{T}\right) \geq 0.4$. The uncertainty in the fit of individual run-periods is large due to lack of statistics. The comparison is only qualitative, and the differences observed are not considered part of the total uncertainty.

\subsubsection{Level 2 Trigger Efficiency (L2_CJET10_JET10)}

At Level 2, two clusters of $10 \mathrm{GeV}$ are required with one being central $(|\eta|<0.9 \mid)$. The thresholds in the preselection are chosen in order to make Level 2 fully efficient 
which is demonstrated in this section. This choice greatly simplifies the treatment of the trigger, because the jet selection otherwise would be correlated with the $\mathbb{E}_{T}$ selection at L1 and L3. The single jet components (JET10 and CJET10) are always satisfied for offline jets with $E_{T}>25 \mathrm{GeV}$. A small efficiency loss is observed due to different angular resolution between L2 clusters and offline jets as well as L2 cluster merging in high occupancy events. This is expressed as a function of the jet-separation angle, $\Delta R$. We require the offline jets to be separated by at least a $\Delta R$ of 1.0 in order to achieve full L2 efficiency.

Figures 4.5 and 4.6 show the efficiency of JET10 and CJET10 as function of offline jet $E_{T}$ for events passing the L1_MET25 requirement and the basic selection in CMUP18 and JET50 data. Jets are selected after removing the trigger biases and requiring that they are "isolated" (i.e. no other jet in a radius of 1.0) to ensure unambiguous jet-to-cluster matching. It is sufficient to require a cut at $25 \mathrm{GeV}$ on the corrected offline jet $E_{T}$ for full efficiency.

The path of the MET35 trigger changed during the $1 \mathrm{fb}^{-1}$ period when one of the clusters was required to be central. However, it did not affect the signal acceptance. In order to unify the treatment of the two paths, we introduce an explicit centrality requirement for one of the offline corrected jets in the entire $1 \mathrm{fb}^{-1}$ data. Figure 4.7 shows the efficiency of L2_CJET10 as a function of $\eta$ for jets with $E_{T}>25 \mathrm{GeV}$. The efficiency is $\sim 100 \%$ when the central jet is within $|\eta|<0.9$. 

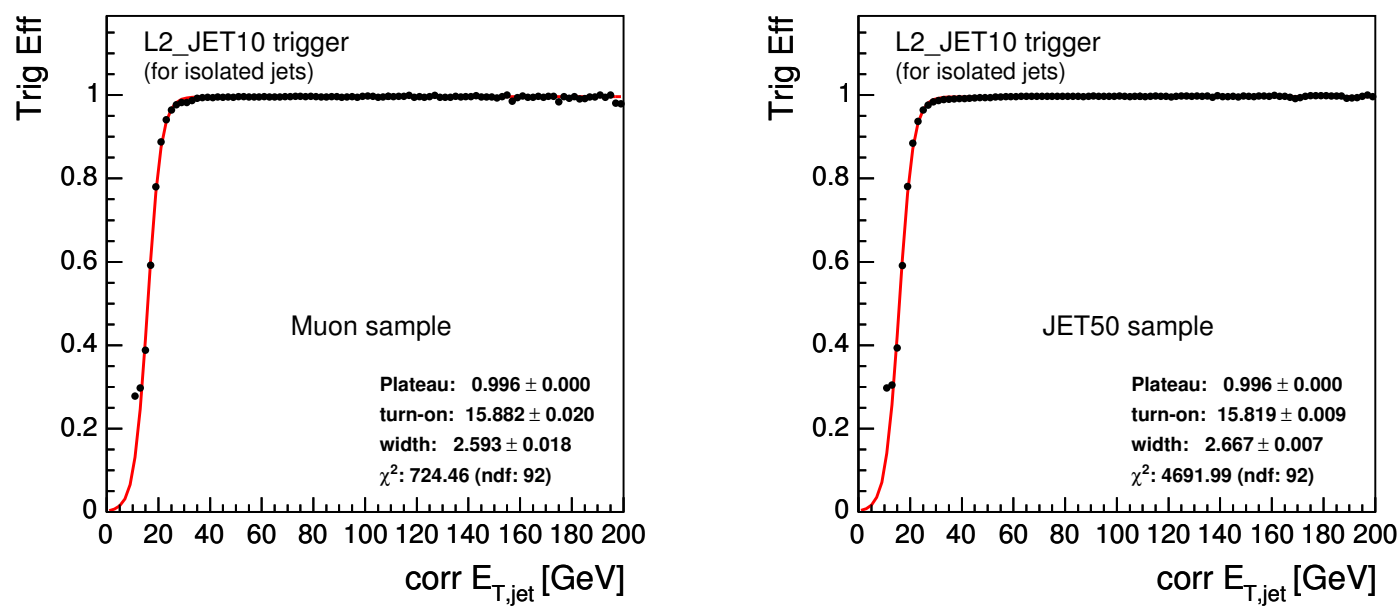

Fig. 4.5. Efficiency of the L2_JET10 requirement as function of the corrected $E_{T}$ of the matching offline jets in the CMUP18 and JET50 data samples.
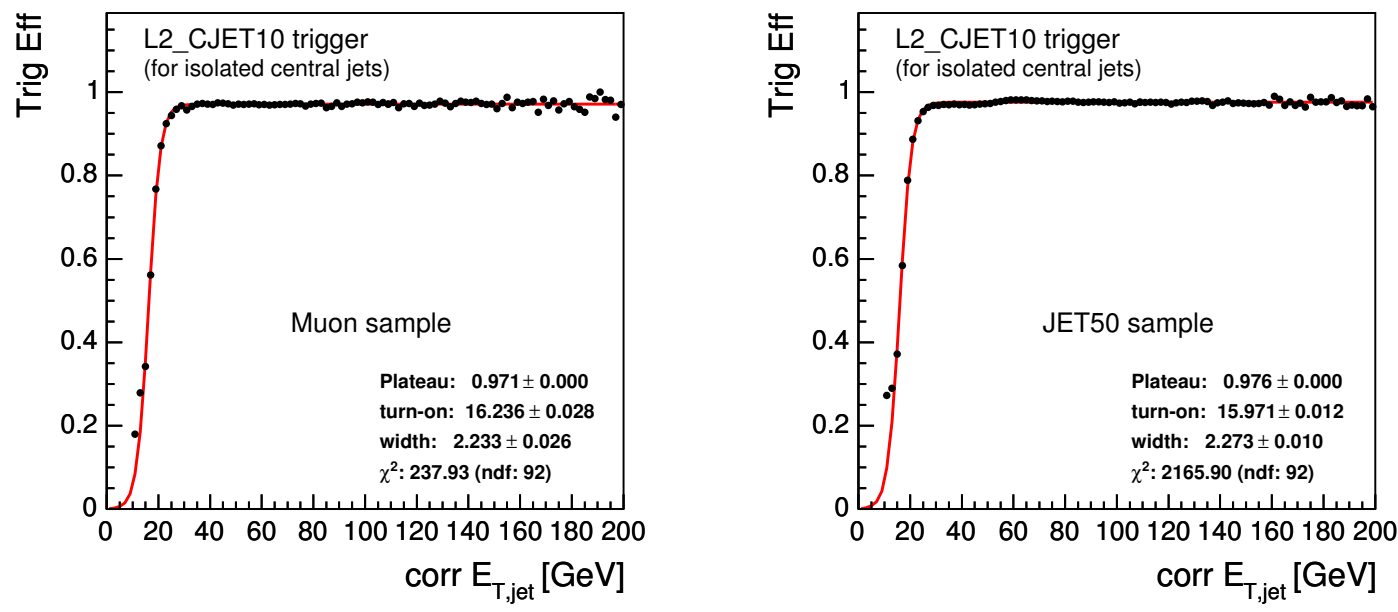

Fig. 4.6. Efficiency of the L2_CJET10 requirement as function of the corrected $E_{T}$ of the matching offline jets in the CMUP18 and JET50 data samples. 

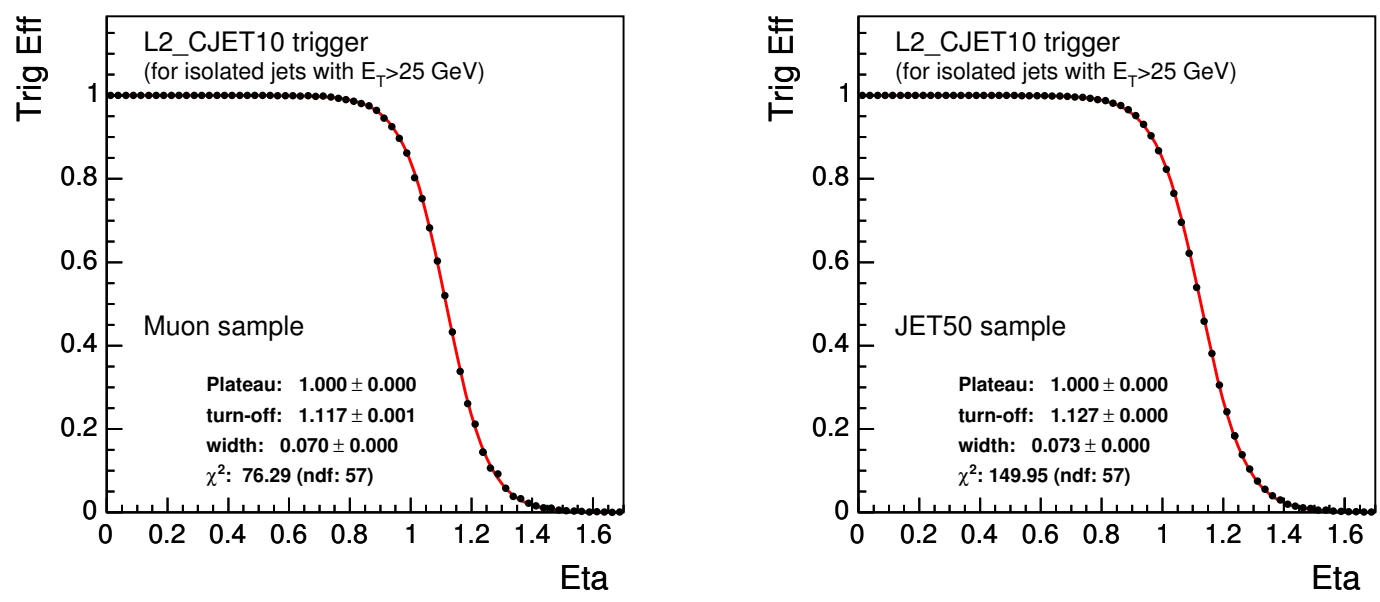

Fig. 4.7. Efficiency of the L2_CJET10 requirement as function of $\eta$ of the matching offline jets in the CMUP18 and JET50 data samples.

The single jet requirement is now fully efficient, but any cluster-merging still has to be removed. Relaxing the condition on $\Delta R$, but keeping the veto on the third jet, we obtain the efficiency curves in Figure 4.8. The efficiency is plotted as function of the distance between the two jets in three data samples. This is a slightly pessimistic estimate of the efficiency since such clean dijet events are very rare due to final state radiation. We conclude that the $\Delta R>1.0$ condition is required.

In order to demonstrate that Level 2 efficiency is essentially $100 \%$, we test it as a function of the dijet invariant mass. Figure 4.9 shows the efficiency of Level 2 after preselection and $\Delta \phi\left(\right.$ jet $\left.2, \notin_{T}\right) \geq 0.4$. Again, it is a pessimistic estimate in that we required the jets to be isolated. 

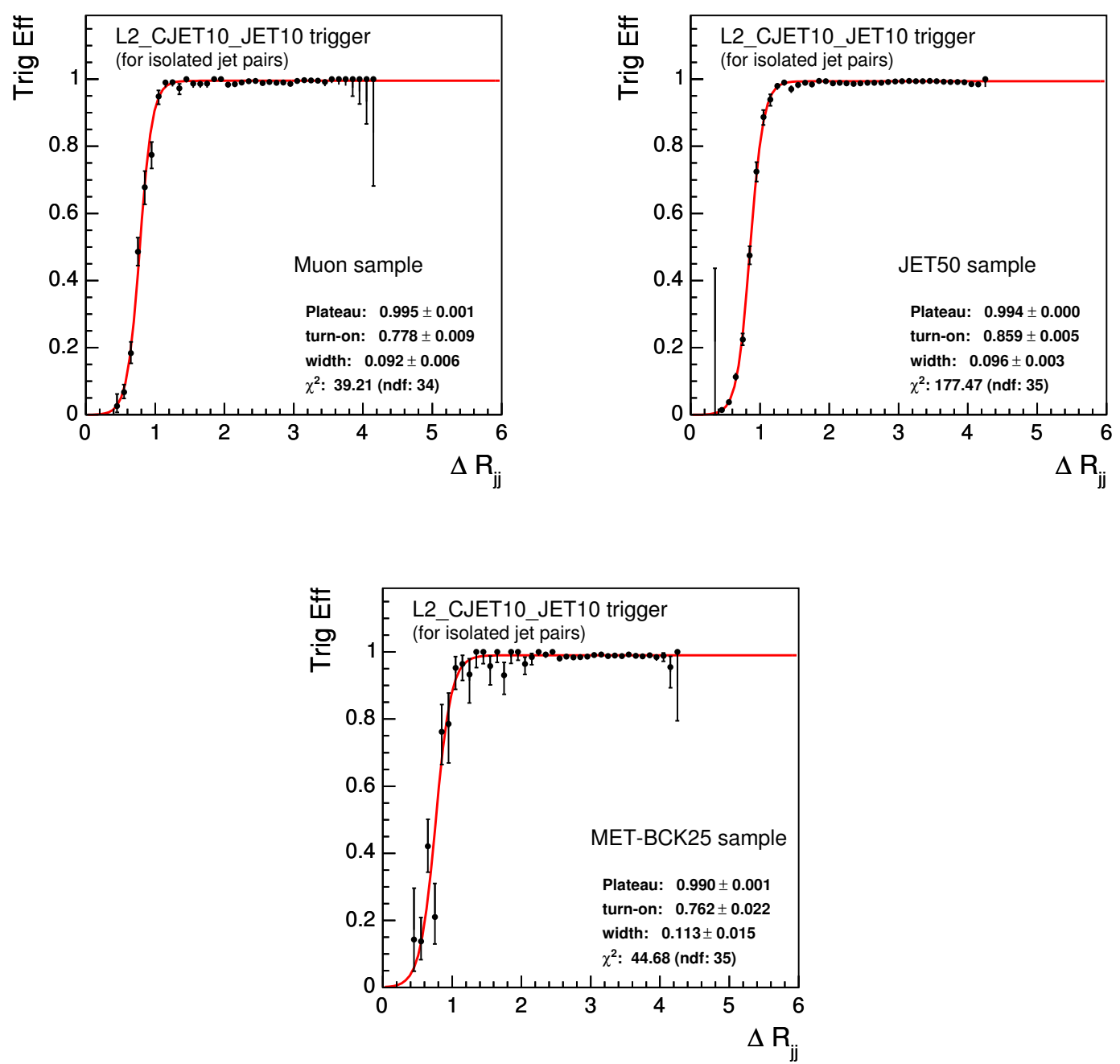

Fig. 4.8. Efficiency of L2_CJET10_JET10 for dijet systems as function of the $\Delta R$ between the two jets in the CMUP18, the JET50, and the MET25 data samples. Jets are required to satisfy the single-jet conditions. 

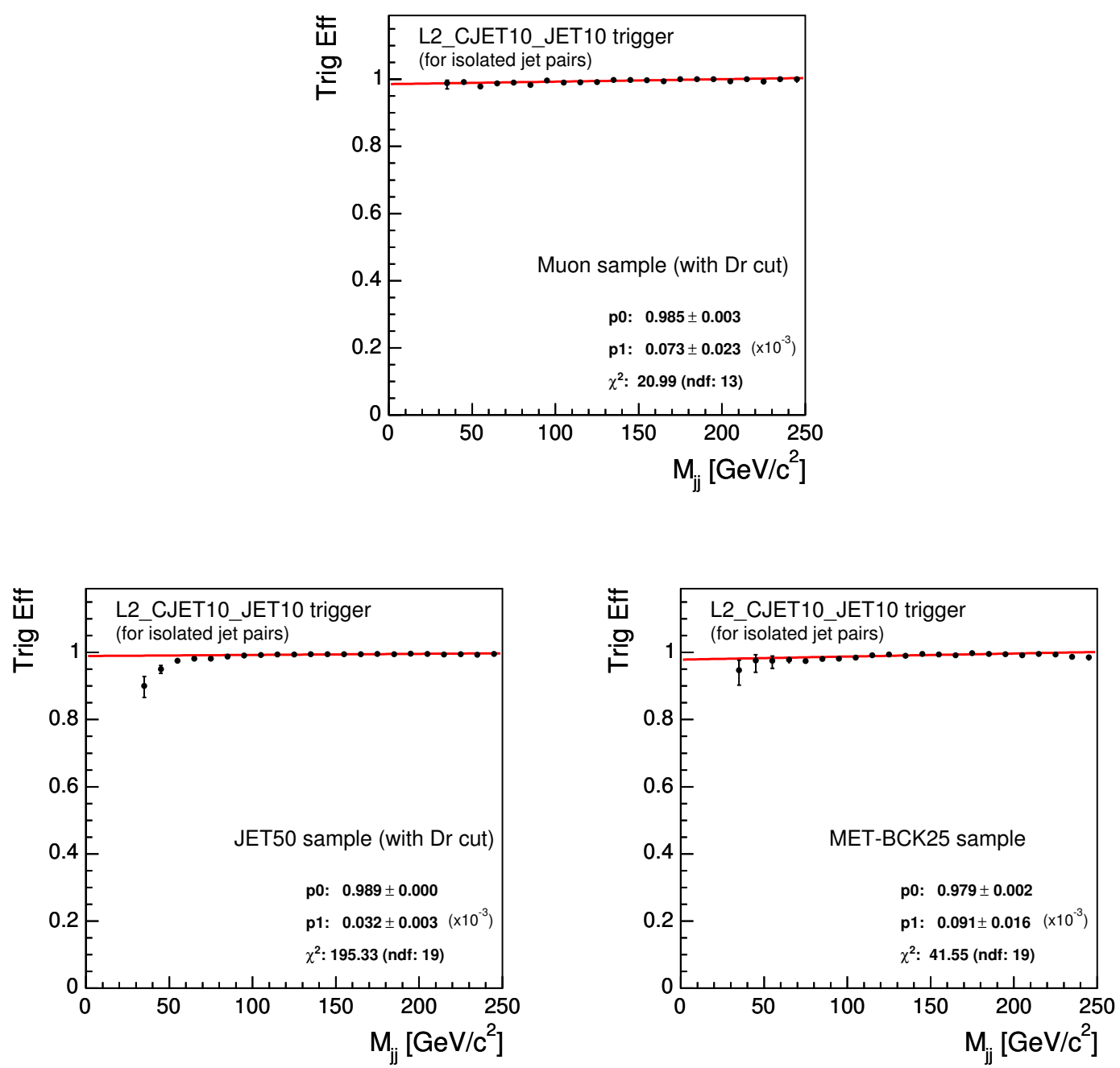

Fig. 4.9. Efficiency of L2_CJET10_JET10 for a dijet system as function of the dijet invariant mass in the CMUP18, the JET50, and the MET25 data samples. Jets are required to satisfy the single-jet conditions. 


\subsubsection{Level 3 Trigger Efficiency (L3_MET35)}

A cut $\mathbb{E}_{T}>35 \mathrm{GeV}$ on the L3-reconstructed missing transverse energy is applied at Level 3. We perform the same test as at Level 1 and obtain the efficiency for events passing the preselection, the Level 1, and Level 2 requirements. The result is shown in Figure 4.10 for the CMUP18, the JET50, and the MET25 data samples before splitting them into $\Delta \phi\left(\right.$ jet $\left.2, \not_{T}\right)<0.4$ and $\Delta \phi\left(\right.$ jet $\left.2, \not_{T}\right) \geq 0.4$.

We expect the turn-on to be different in events where the $\mathbb{E}_{T}$ is produced by jet energy mismeasurement from those with real $\not_{T}$. Figure 4.11 shows the results when $\Delta \phi\left(\right.$ jet $\left.2, \notin_{T}\right)<0.4$ and Figure 4.12 when $\Delta \phi\left(\right.$ jet $\left.2, \notin_{T}\right) \geq 0.4$.

The L3 trigger efficiency as a function of $\mathscr{E}_{T}$ is slightly different in the different samples. We assign an uncertainty in the turn on region that covers this effect. The uncertainty in the plateau is small, but we quote an overall $1 \%$ to account for uncertainties in the beginning of the plateau. The efficiencies and systematic uncertainties are assigned the following way:

- When $\Delta \phi\left(\right.$ jet $\left.2, \notin_{T}\right)<0.4$ :

$$
\epsilon_{L 3}\left(\not_{T}\right)=\frac{1}{1+\exp \left(\frac{39-\not \phi_{T}}{6.0}\right)} ;
$$

and the relative uncertainty:

$$
\frac{\Delta \epsilon}{\epsilon}\left(\notin_{T}\right)= \begin{cases}0.01+0.004 *\left(60-\notin_{T}\right) & \text { if } \notin_{T}<60 \mathrm{GeV}, \\ 0.01 & \text { if } \notin_{T} \geq 60 \mathrm{GeV} .\end{cases}
$$

- When $\Delta \phi\left(\right.$ jet $\left.2, \notin_{T}\right) \geq 0.4$ :

$$
\epsilon_{L 3}\left(\not \epsilon_{T}\right)=\frac{1}{1+\exp \left(\frac{42-\not \phi_{T}}{4.0}\right)} ;
$$

with the same relative uncertainty as in the first case. 

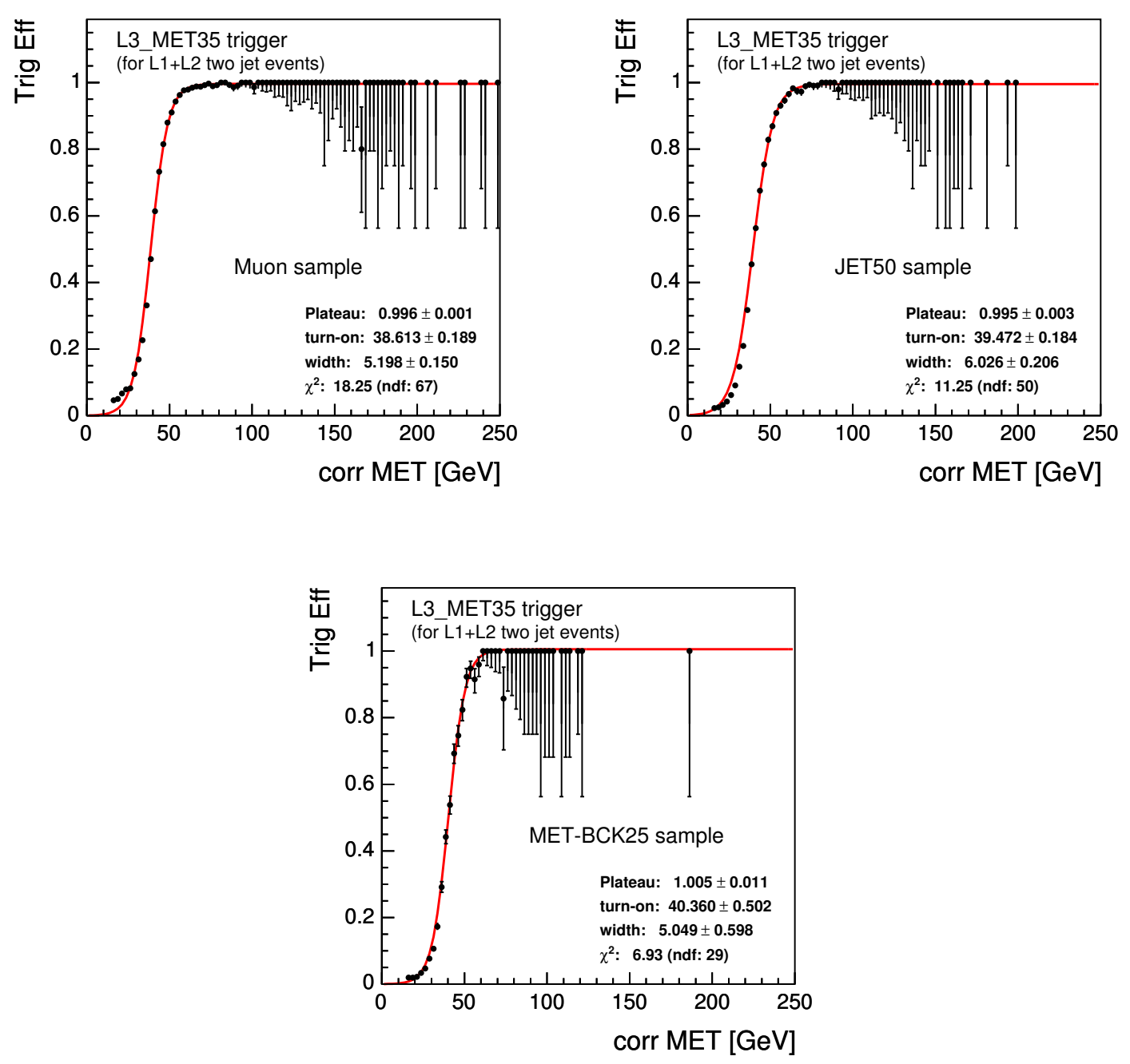

Fig. 4.10. Efficiency of L3_MET35 as function of the corrected $\notin_{T}$ in the CMUP18, JET50 and the MET25 samples for events passing the preselection, the Level 1 and Level 2 triggers. 

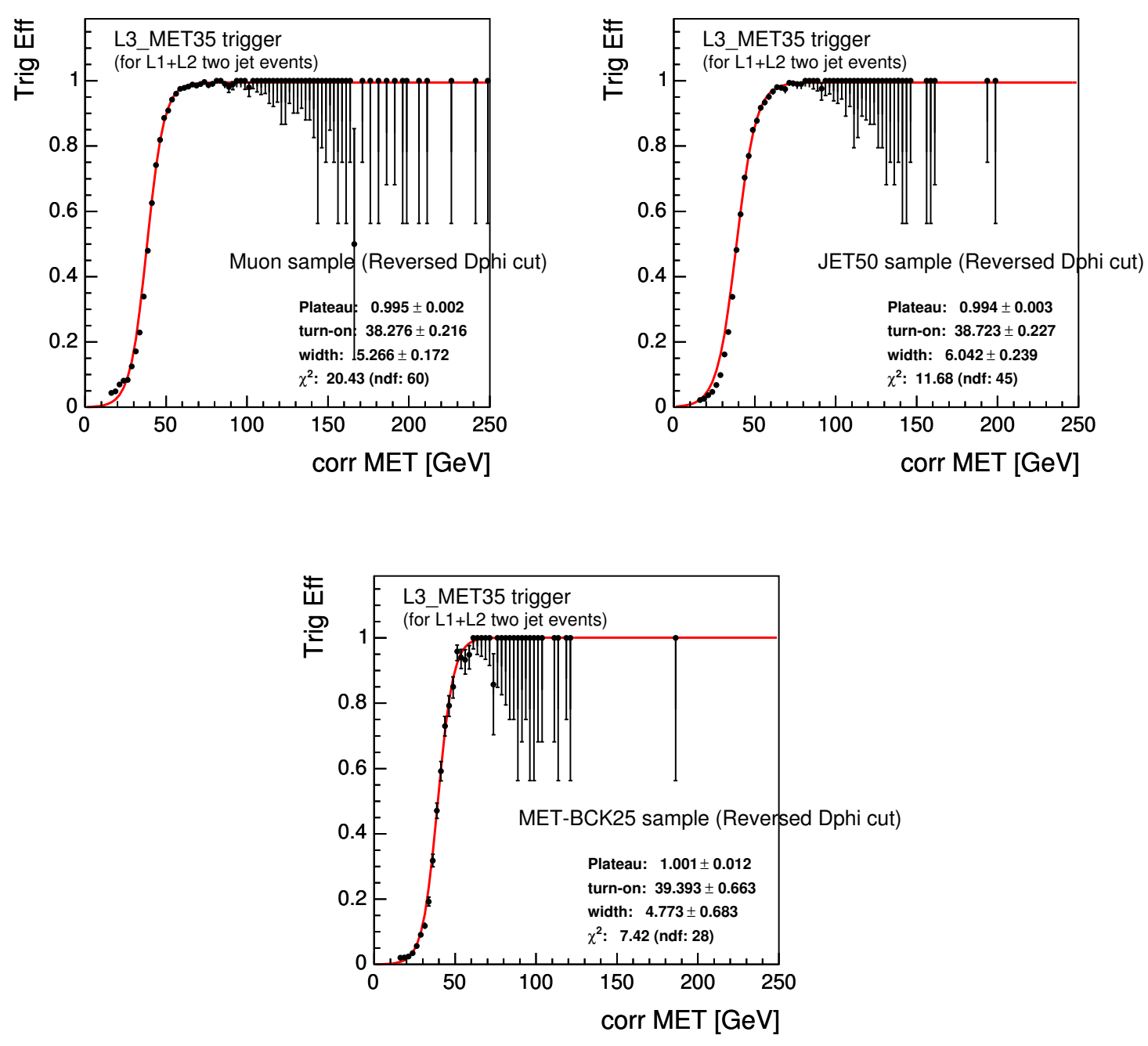

Fig. 4.11. Efficiency of L3_MET35 as function of the corrected $\not_{T}$ in the CMUP18, JET50 and the MET25 samples for events passing the preselection, the Level 1 and Level 2 triggers, and $\Delta \phi\left(\right.$ jet2, $\left.\notin_{T}\right)<0.4$ 

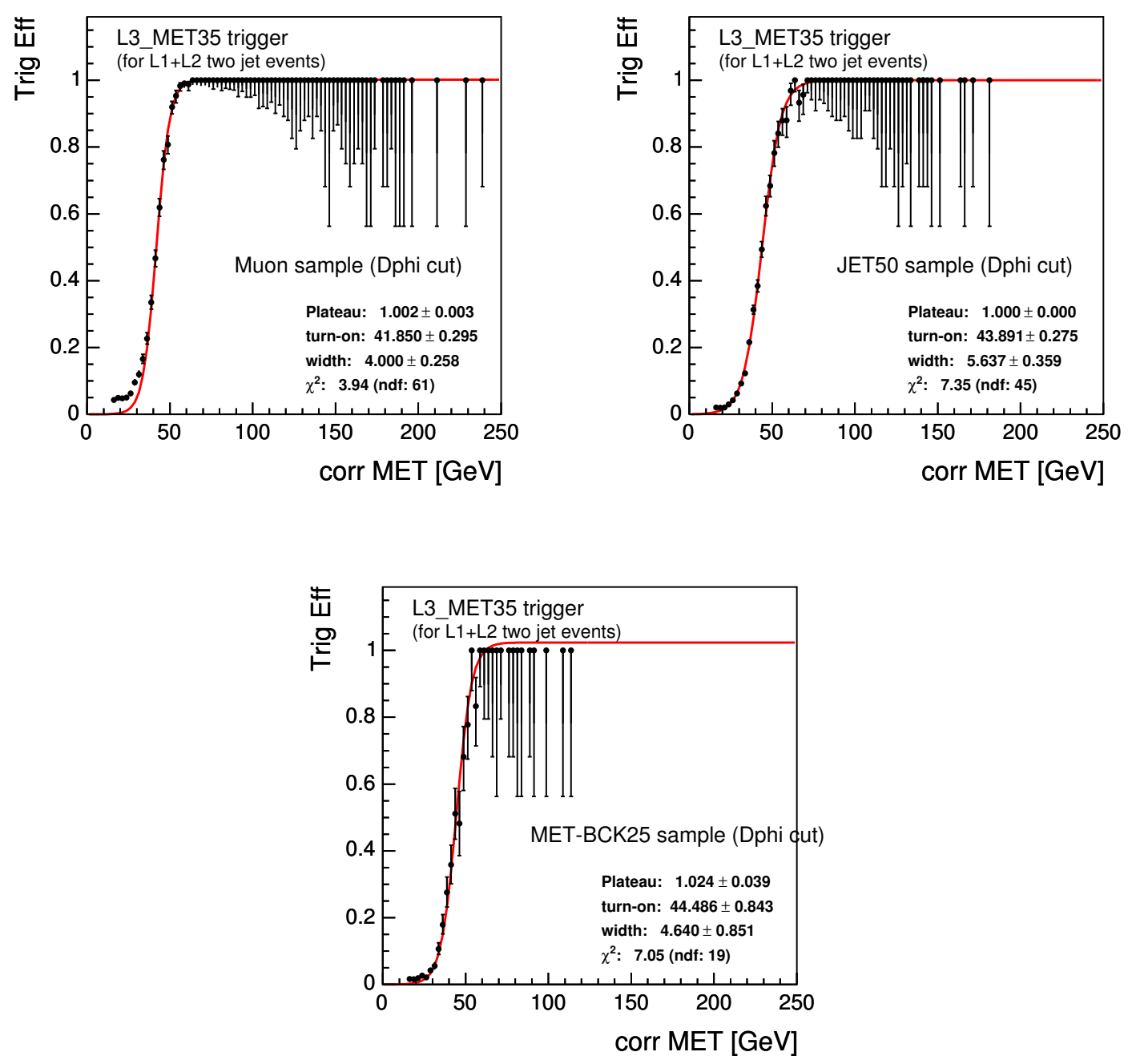

Fig. 4.12. Efficiency of L3_MET35 as function of the corrected $\not_{T}$ in the CMUP18, JET50 and the MET25 samples for events passing the preselection, the Level 1 and Level 2 triggers, and $\Delta \phi\left(\right.$ jet2, $\left.\notin_{T}\right) \geq 0.4$ 


\section{SIGNAL AND BACKGROUND SIMULATION}

\subsection{Signal Monte Carlo}

The $Z H / W H$ signal samples are generated with Pythia Monte Carlo [46] and simulated with the CDF Monte Carlo software which includes run-dependent effects. Multiple interactions are simulated as a number of minimum bias events that is proportional to the average instantaneous luminosity in each run. The production output is ntuplized to CDF Standard Ntuples built in ROOT [47].

Table 5.1 lists the mass points at which the Higgs bosons are generated, the branching fraction of the Higgs boson to b-quarks, and the corresponding $\sigma(Z H)$. $\operatorname{Br}(Z \rightarrow \nu \bar{\nu}, H \rightarrow b \bar{b})$ or $\sigma(W H) \cdot \operatorname{Br}(W \rightarrow l \nu, H \rightarrow b \bar{b})$ effective cross-sections. In these samples the Higgs is forced to decay into b-jet pairs, the Z-boson to neutrinos, and the $W^{ \pm}$to leptons.

Table 5.1

Cross-sections of the $\mathrm{ZH} / \mathrm{WH}$ processes at various Higgs masses with the branching fractions $\operatorname{Br}(Z \rightarrow \nu \bar{\nu})=0.20$ and $\operatorname{Br}(W \rightarrow l \nu)=0.32$ applied (i.e. number of Higgs produced in $1 \mathrm{fb}^{-1}$ data)

\begin{tabular}{|c|c|c|c|}
\hline Higgs Mass $(\mathrm{GeV})$ & $\operatorname{Br}(H \rightarrow b \bar{b})$ & $\sigma(Z H) \cdot \operatorname{Br}(\nu \bar{\nu}, b \bar{b})(\mathrm{fb})$ & $\sigma(W H) \cdot \operatorname{Br}(l \nu, b \bar{b})(\mathrm{fb})$ \\
\hline \hline 110 & 0.770 & 19.19 & 53.37 \\
115 & 0.732 & 15.78 & 43.50 \\
120 & 0.679 & 12.70 & 34.70 \\
125 & 0.610 & 9.92 & 26.89 \\
130 & 0.527 & 7.49 & 20.14 \\
135 & 0.436 & 5.43 & 14.46 \\
140 & 0.344 & 3.76 & 9.93 \\
\hline
\end{tabular}




\subsection{Background Simulation}

In the signal events the Higgs decays into two b-jets, the Z-boson into two neutrinos, and the $\mathrm{W}$ to leptons. The most important characteristics of these events are the large intrinsic missing transverse energy, relatively low jet multiplicity, and the lack of (detectable) isolated leptons. There are numerous Standard Model processes that can produce this signature. In this section, we list all backgrounds considered in the analysis (see Table 5.2). Most of the background simulations were filtered at the generator level for events that contain a b- or a c-quark and have $\mathbb{E}_{T}>25 \mathrm{GeV}$ after event reconstruction. The first column lists the number of events before filtering, the second the filter efficiency. The cross-sections correspond to the inclusive production before the filter was applied.

\subsubsection{QCD Multi-jet Background}

The most significant background at the preselection stage of the analysis is represented by QCD multi-jet processes. QCD jet production has large cross-section $(\sim \mu \mathrm{b})$, which is about 9 orders of magnitude greater than the signal before requiring any b-tags. Although, these processes generally do not contain high- $p_{T}$ neutrinos, mismeasured jets cause imbalance in the total transverse energy. Light flavor (uds) QCD events can pass the basic selection cuts if one of the jets is mistagged. QCD heavy flavor pair production yields taggable jets and large $\mathbb{E}_{T}$ if a b- or c-quark undergoes a semi-leptonic decay. In both cases, the missing transverse energy tends to be aligned parallel or anti-parallel to the first or second most energetic jet. This characteristic provides the most effective strategy to reduce the QCD heavy flavor background.

Due to the large cross-section, it is practically impossible to generate enough statistics to simulate all QCD processes. In this analysis, we simulate processes which yield real taggable objects, that is, when a b- or a c-quark pair is created. The rest of the QCD processes are estimated using the heavy flavor mis-identification 
Table 5.2

Monte Carlo background samples. Cross-sections are already NLO where the $\mathrm{k}$-factor is not specified

\begin{tabular}{|l|c|c|c|c|c|}
\hline Process & $\begin{array}{c}\text { Events } \\
\text { before } \\
\text { filtering }\end{array}$ & $\begin{array}{c}\text { Filter } \\
\text { eff. } \\
(\%)\end{array}$ & $\begin{array}{c}\text { Inclusive } \\
\text { cross-sec. } \\
(\mathrm{pb})\end{array}$ & $\begin{array}{c}\text { NLO } \\
\text { k-factor }\end{array}$ & $\begin{array}{c}\text { Luminosity } \\
\left(\mathrm{fb}^{-1}\right)\end{array}$ \\
\hline \hline QCD mutli-jet & $1528 \mathrm{M}$ & 1.7 & $4.4 \times 10^{5}$ & $\sim 1.4$ & $\sim 2.5$ \\
\hline$t \bar{t}$ & $988 \mathrm{~K}$ & 100 & 7.3 & & 135.4 \\
single top (s-channel) & $254 \mathrm{~K}$ & 100 & 0.88 & & 289 \\
single top (t-channel) & $259 \mathrm{~K}$ & 100 & 1.98 & & 131 \\
\hline$W^{ \pm}$to $e^{ \pm} \nu$ & $30.3 \mathrm{M}$ & 9.1 & 1959 & 1.4 & 11.0 \\
$W^{ \pm}$to $\mu^{ \pm} \nu$ & $56.3 \mathrm{M}$ & 1.1 & 1982 & 1.4 & 20.3 \\
$W^{ \pm}$to $\tau^{ \pm} \nu$ & $26.3 \mathrm{M}$ & 20.2 & 1971 & 1.4 & 9.54 \\
\hline Z to $e^{-} e^{+}$ & $10.4 \mathrm{M}$ & 6.3 & 355 & 1.4 & 21.0 \\
$\mathrm{Z}$ to $\mu^{-} \mu^{+}$ & $10.4 \mathrm{M}$ & 6.3 & 355 & 1.4 & 21.0 \\
Z to $\tau^{-} \tau^{+}$ & $5.1 \mathrm{M}$ & 8.7 & 355 & 1.4 & 10.3 \\
$\mathrm{Z}$ to $\nu \bar{\nu}$ & $62.5 \mathrm{M}$ & 0.8 & 1102 & 1.4 & 40.5 \\
\hline WW to all & $519 \mathrm{~K}$ & 100 & 12.0 & & 43.3 \\
WZ to all & $520 \mathrm{~K}$ & 100 & 3.64 & & 143 \\
ZZ to all & $521 \mathrm{~K}$ & 100 & 3.32 & & 157 \\
\hline
\end{tabular}

probability after requiring a positive tag in the selection. A jet in any MC sample is considered to be tagged only if there is a heavy flavor quark inside the $R=0.4$ radius cone of the jet in order to avoid double-counting the mistags. This condition is not so much relevant as long as all Monte Carlo samples are heavy flavor filtered. The mistags estimation is explained in Section 5.2.4.

We have generated over a billion events, corresponding to about $2.5 \mathrm{fb}^{-1}$ data, with $p_{\text {Tmin }}>50 \mathrm{GeV}$ which we filtered for b- and c-quarks. These events were 
simulated, reconstructed and passed through another filter of offline uncorrected $\mathbb{E}_{T}>25 \mathrm{GeV}$. The $\not_{T}$ cut applied at reconstruction level is $100 \%$ efficient after the preselection requiring the corrected $\notin_{T}>50 \mathrm{GeV}$.

The product of the Pythia LO cross-section corrected with the filter efficiency and a k-factor of 1.4 was initially used to normalize the sample. Since the exact NLO cross-section of the dijet heavy flavor production is not well known, we scale the number of QCD events to match the data by adjusting the k-factor. The choice of the initial k-factor is the same as in the $\mathrm{W} / \mathrm{Z}$ background. It is also supported by earlier QCD normalization studies, thus we expect it to be close to the appropriate value. It is applied before determining the final normalization in order to reduce the number of iterations required in the calculation to a single step. See Section 6.3 for further details on the normalization method.

Adjusting the k-factor makes comparisons with similar analyses possible and provides a cross-check of the normalization with earlier studies. We do not intend to measure the actual NLO correction! The LO prediction of Pythia is simply a natural choice for a common denominator without which the normalization factor would be just the ratio between the number of events required to match the data and the number of generated events. This ratio would keep changing as more simulation is produced. By normalizing to data, we lump various simulation effects into the kfactor. For example, the normalization is done in the single and double tag samples separately. The double tag sample is dominated by b-production, but the single tag still contains a substantial fraction of charm events. We implicitly correct the simulation of the heavy flavor fraction by normalizing the single and double tags separately.

\subsubsection{Top Production}

Two classes of top-production are considered in this analysis. The pair-production and the single top production in the t- and s-channels. They all yield a measurable contribution to the background in the signal region, but the pair-production is the 
most significant. The single top background becomes more relevant after requiring only two hard jets in the event. Due to its large mass and the semi-leptonic decay of the top, these events are energetic, have large $\not_{T}$ and high jet multiplicity.

The $t \bar{t}$ events were generated with Pythia with a top quark mass of $171.5 \mathrm{GeV}$ and a $7.3 \pm 0.8 \mathrm{pb}$ cross-section which correspond to the current CDF averages [48]. The single top samples were generated at $178 \mathrm{GeV}$, and the theoretical production cross-sections $0.88 \pm 0.11 \mathrm{pb}$ in the s-channel and $1.98 \pm 0.25 \mathrm{pb}$ in the t-channel were used $[49,50]$. In the latter case, a $11.5 \%$ systematic uncertainty was assigned to the cross-section.

\subsubsection{Di-boson and $\mathrm{W} / \mathrm{Z}$ Backgrounds}

The electroweak backgrounds are all generated with Pythia. All the $\mathrm{W}$ and Z samples are filtered for a b- or c-quark at generator level with the exception of $W \rightarrow \tau \nu$ and $Z \rightarrow \tau \bar{\tau}$. A $\notin_{T}>25 \mathrm{GeV}$ filter is also applied after event reconstruction whenever it leads to significant reduction in the number of generated events.

Both the $\mathrm{Z}+$ h.f. and $\mathrm{W}+$ h.f. backgrounds are normalized using the inclusive cross-sections measured by the Electroweak Working Group [51]. Our samples are identical to the ones used in their measurements with the exception of the filters. We correct the number of generated events by the filtering efficiencies. The measured cross-sections correspond to a k-factor of 1.4 with respect to the Pythia LO predictions. There are no measured values for $W \rightarrow \tau \nu$ and $Z \rightarrow \tau \bar{\tau}$, therefore we use the LO Pythia cross-section scaled by 1.4.

The Z/W samples are properly normalized to predict the yield of the inclusive production. However, the heavy flavor production in the Pythia simulation provides a possible source of systematic error. We assign a $40 \%$ uncertainty based on the total error of the $\mathrm{Z}+$ h.f. cross-section measurement at CDF. The analysis presented in [52] measures the cross-section ratio between inclusive $Z$ and $Z+$ h.f. processes and finds that the LO Pythia prediction scaled by $\mathrm{k}=1.38$ reproduces well the $\mathrm{Z}+\mathrm{h}$.f. 
observed in data. This analysis also used a b/c-filtered Pythia simulation identical to ours.

In the diboson simulations, the bosons decay inclusively. We use LO Pythia crosssections scaled by k-factors. The k-factors were obtained from the ratios between the NLO and LO cross-section predicted in MCFM [53]. The LO MCFM predictions are also consistent with those from Pythia and are reported to be in good agreement with the data. An 11.5\% uncertainty on the MCFM cross-section is assigned for the diboson normalization [54].

\subsubsection{Mistagged Light Flavor Background}

The tagging efficiency of the SecVtx algorithm for light flavor jets is a few per cent depending on which setting of SecVtx is used (see definition of tags in Section 3.5). These light flavor tags are called mistags. Although the mistag efficiency is an order of magnitude smaller than the heavy flavor tagging efficiency, the large crosssection of processes that produce light flavor jets make the mistag background one of the largest in the single-tag data sample (single mistags). The significance of the mistag background is reduced after requiring two tags (double mistags). We estimate mistags by applying the Mistag matrix $R_{\text {mistag }}^{+}$(Equation 5.4) on data.

The Mistag matrix is a six-dimensional matrix with elements that predict the probability of light flavor jets to be tagged by the SecVtx algorithm. The tagging probability of a jet depends on its raw $E_{T}, \eta$, and number of good tracks $\left(N_{t r k}^{\text {good }}\right)$, and the $\sum E_{T}$, vertex number, and primary vertex position of the event in which the jet is found. One obtains the rate at which events are mistagged by summing the mistag probability of all taggable jets in those events. The matrix elements are computed with the formula in Equation (5.4). A description on using the matrix is given in [55].

In this analysis, events are classified into exclusive single and double tag categories. The inclusive single mistag is estimated by running on a pre-tag sample with total light and heavy flavor events $N_{\text {light }}^{\text {pre }}+N_{\text {heavy }}^{\text {pre }}$. The pre-tag data consists of events that 
pass all relevant selection cuts without any tag requirements. Double mistags are estimated from the same data after requiring one observed positive tag. This predicts the rate at which the non-tagged jet produces a second tag that is a mistag. The exclusive single mistag is the difference between the inclusive single mistag and the double mistag predictions.

It is generally not known if a positive tag is real or a mistag; therefore, it is not possible to construct a mistag matrix directly from data. Since negative tags are always fake, the construction of the mistag matrix starts by creating a negative tag matrix $R^{-}$defined as in Equation (5.1) where $N_{\text {light }}^{-}+N_{\text {heavy }}^{-}$is the number of negative tags in the data.

$$
R^{-}=\frac{N_{\text {light }}^{-}+N_{\text {heavy }}^{-}}{N_{\text {light }}^{\text {pre }}+N_{\text {heavy }}^{\text {pre }}}
$$

Negative tags are mainly due to resolution effects in the tracking. The majority of the mistags (light flavor positive tags) are produced similarly. The rest comes from physical sources, for example long-lived particle decays $\left(K_{s}\right.$ or $\left.\Lambda\right)$ and interactions in the beam-pipe or with the detector material. These processes enhance the mistag rate with respect to the the negative tag rate. We correct for these effects by multiplying the negative tags with an asymmetry factor $\alpha$. In practice, this is done right within the Mistag matrix as shown in Equation (5.4).

The method that was used to compute the mistag asymmetry is described in note [56]. Templates of signed tag mass distributions obtained from Monte Carlo simulations of light and heavy flavor jets are fitted to the tag mass observed in the data. The fit provides normalization for the various light and heavy flavor jet productions and fixes the heavy flavor fraction in the simulation. It is not possible to fit both the negative and the positive tag mass distributions simultaneously, because the Monte Carlo underestimates the fraction of negative tags with respect to the positive ones. In other words, it provides a too optimistic description of the detector resolution. The positive tag excess over the negative tags, however, is physically motivated and expected to be better reproduced by the simulation. It is reasonable to assume that the simulation underestimates the part of the mistag rate which is due to resolu- 
tion effects as much as the negative tag rate; therefore, the fit is done in two steps. In the first step, the negative templates are subtracted from the positive ones in order to get templates for the positive tag excess. The sum of these Monte Carlo templates is fitted to the data, and the correct normalization for the simulations is computed. In the second step, the negative templates are fitted to the data such that the relative fractions of the various flavors are kept the same as measured in the first step. The resulting overall scale factor is called the Negative Scale Factor, and it is assumed to be the same in all Monte Carlo processes regardless of the flavor. The second fit is required to obtain the number of mistags that were subtracted in the first step. The mistag asymmetry is defined as the ratio between the number of positively tagged light flavor jets in the simulation and the sum of all the negative tags:

$$
\alpha=\frac{N_{\text {light }}^{+}}{N_{\text {light }}^{-}+N_{\text {heavy }}^{-}}
$$

where $N_{\text {light }}^{+}$is the number of mistag jets.

This definition of $\alpha$ still contains the heavy flavor contribution to the negative tags. By scaling the negative tags only with $\alpha$ in order to estimate mistags, one introduces an uncertainty due to possible differences in the flavor compositions between the generic jet sample from which the matrix was produced and the analysis sample in which the matrix is applied. This uncertainty is small for single mistags. However, the first real tag requirement in the double mistag estimation enhances the heavy flavor fraction. In order to get the right prediction in both single and double tags, another scale factor is applied on the top of the asymmetry factor that cancels the heavy flavor contribution in the sample where the mistag matrix was produced:

$$
\beta=\frac{N_{\text {light }}^{\text {pre }}+N_{\text {heavy }}^{\text {pre }}}{N_{\text {light }}^{\text {pre }}}
$$

Thus the elements of the mistag matrix is the product $\alpha \beta R^{-}$.

$$
R_{\text {mistag }}^{+}=\alpha \times \beta \times R^{-}=\frac{N_{\text {light }}^{+}}{N_{\text {light }}^{\text {pre }}}
$$


Consequently, this operator is no longer applicable on the entire pre-tag sample. The heavy flavor contribution should be removed from the pre-tag data before applying the matrix. This is done indirectly by applying the matrix on the heavy flavor simulation and subtracting the result from the total prediction obtained in the data. This correction is often not significant with respect to the systematic uncertainties that are generally considered in this analysis. The $t \bar{t}$ process, for example, is corrected by $5 \%$ in the single tagged and $8 \%$ in the double tagged events. The mistags attributed to the QCD multi-jet production however undergo a much bigger correction (around 15\% to 30\%). This correction has an influence on the QCD normalization. 


\section{CONTROL AND SIGNAL REGIONS}

We are searching for the Standard Model Higgs boson in events with $\not_{T}$ and jets originating from b-quarks. The properties and the expected cross-sections of such Higgs events are predicted by the theory that was introduced in Chapter 1. Since the mass of the Higgs boson is not known, we perform the analysis in a set of possible mass-points. The list of these mass-points and the corresponding predicted crosssections are shown in Table 5.1. The lowest point is dictated by the LEP II direct search limit of $114.4 \mathrm{GeV}$. The highest point was selected at a mass of $140 \mathrm{GeV}$, since above this value less than a third of the Higgs bosons decay to b-quarks while other decay channels open and become more sensitive to the discovery of the Higgs (see Figure 1.3).

The small effective cross-section of the Higgs production represents the greatest challenge in this analysis. Although, the theoretical prediction for the cross-section is not explicitly used in the search, it allows us to estimate the number of events expected in $1 \mathrm{fb}^{-1}$ data if the SM Higgs exists. These events yield a very small signal over a much larger background. We use the signal simulation to enhance the $S / \sqrt{B}$ ratio by applying selections that are favorable for the signal. In order to maximize $S / \sqrt{B}$, however, we do not actually need to know the exact cross-section.

Since we are looking for a signal of unknown and possibly very small size, we cannot compare data with the signal simulation directly in the region where the signal is expected (Signal Region, or SR). If we were to do so, we would risk the results being influenced by our expectations (experimenter's bias). Thus there is no way of telling directly how well the simulation reproduces the observables in Higgs events that are used in the selection. The cross-check has to be done indirectly by defining Control Regions (CR) where signal is not expected and comparing the observables via the Standard Model background predictions. The comparison is successful if the 
expected background agrees with the observed data within the systematic uncertainties assigned to the observables. When the background is proved to be correctly

reproduced in the control regions, the $S / \sqrt{B}$ optimization is performed "blindly" on the signal and background simulations in the Signal Region.

In the last step of the analysis, we evaluate the uncertainties on the signal and the background in the Signal Region and compare the background prediction with the observed data. If no significant difference (i.e. excess) is found, we set $95 \%$ C.L. limits on the signal. An overview of systematic uncertainties is given in Section 6.7. The limit calculation is described in Chapter 7 .

\subsection{Preselection}

In the first step of processing both data and simulation, a set of filters are applied to select events for analysis:

- Good-run selection (Section 3.7)

- Trigger selection, only applied on data (Section 4.1)

- Quality cuts developed for $\mathbb{H}_{T}$-triggered events (Section 3.2.2)

- Preselection cuts after correcting jet $E_{T}$ and $\notin_{T}$. These cuts are motivated by the trigger efficiency study (Section 4.2)

Events passing these selection criteria are divided into control regions and signal region. The preselection cuts are defined by considering the properties of the signal and the trigger dataset.

One conclusion from the trigger study was that the efficiency turn-on parametrization strongly depends on the physical process producing the analyzed events. The systematic uncertainties due to the choice of the trigger sample used in the efficiency calculations are unacceptably large at small $\notin_{T}$. In order to reduce this uncertainty, we require every event to have $\mathbb{E}_{T}$ above $50 \mathrm{GeV}$. This is not expected to lead to 
any considerable sensitivity loss because the fraction of signal events at small $\notin_{T}$ is negligible with respect to the exponentially rising QCD multi-jet background. The jet-selection must be fully efficient in order to use the $\mathbb{E}_{T}$-based efficiency parameterization. The trigger efficiency of the two jet selection is $100 \%$ if the leading jet $E_{T}$ is above $35 \mathrm{GeV}$, the second leading jet is above $25 \mathrm{GeV}$, and one jet is central $(|\eta|<0.9)$.

We require events to contain only two jets with corrected $E_{T}>20 \mathrm{GeV}$ within $|\eta|<2.4$ and discard events containing further jets to suppress the QCD multi-jet and top backgrounds. The remaining two jets are required to be within $|\eta|<2.0$ which is where the Higgs jets are expected.

The last step of the preselection is to discard events with no positively b-tagged jets. We use the SecVtx tight tagger algorithm (Section 3.5). A jet is considered b-tagged if it is positively tagged by the SecVtx algorithm, and

- $E_{T}$ is above $20 \mathrm{GeV}$

- $|\eta|<2.0$

- in case of Monte Carlo events, a "taggable" particle must be found in the 0.4 radian cone around the axis of the jet. A taggable particle is a b-, a c-quark, a $\tau$ lepton if that originates from a primary $\mathrm{W}$ or $\mathrm{Z}$ boson, or a top quark.

Summary of the preselection cuts:

- $\mathbb{E}_{T}{ }^{\text {Corr }}>50 \mathrm{GeV}$

- Exactly two jets with $E_{T}^{\text {Corr }}>20 \mathrm{GeV}$ and $|\eta|<2.4$

- Jet $E_{T}^{1}>35 \mathrm{GeV}$ and $|\eta|<2.0$

- Jet $E_{T}^{2}>25 \mathrm{GeV}$ and $|\eta|<2.0$

- One of the leading jets is within $|\eta|<0.9$

- At least 1 positive b-tag 


\subsection{Definition of Signal and Control Regions}

The most important property of the QCD multi-jet events is that the $\not_{T}$ tends to be parallel or anti-parallel to the most energetic (leading) or the second to most energetic (second leading) jet. In the signal, this angle peaks around $120^{\circ}$; therefore, cutting on this variable yields excellent separation between the signal and the QCD background. QCD multi-jet processes do not produce isolated leptons. By vetoing them, we can separate this process from most of the other Standard Model backgrounds. The other SM backgrounds produce isolated leptons, with the exception of $\mathrm{Z}$ decaying into neutrinos. These backgrounds are cross-checked in a second control region.

The definition of the control regions and the signal region is as follows (see Fig.6.1):

- Control Region 1 (QCD dominated)

- All leptons are vetoed using the loose lepton identifications described in the next section

- Azimuthal angular separation $\varphi\left(2^{\text {nd }}\right.$ jet,$\left.\notin_{T}\right) \leq 0.4$

- Control Region 2

- At least 1 loose lepton is required

- Azimuthal angular separation $\varphi\left(2^{\text {nd }}\right.$ jet,,$\left.\notin_{T}\right)>0.4$

- Signal Region

- Leptons are vetoed

- Azimuthal angular separations $\varphi\left(2^{\text {nd }}\right.$ jet, $\left.\not_{T}\right)>0.4$ and $\varphi\left(1^{\text {st }} j e t, \not_{T}\right)>0.4$ (see Section 4.2)

The Signal Region is defined first with loose cuts to ensure that the optimization is performed on a totally "blind" region. Table 6.6 shows the reduction of signal and background events in the Signal Region before optimization. 


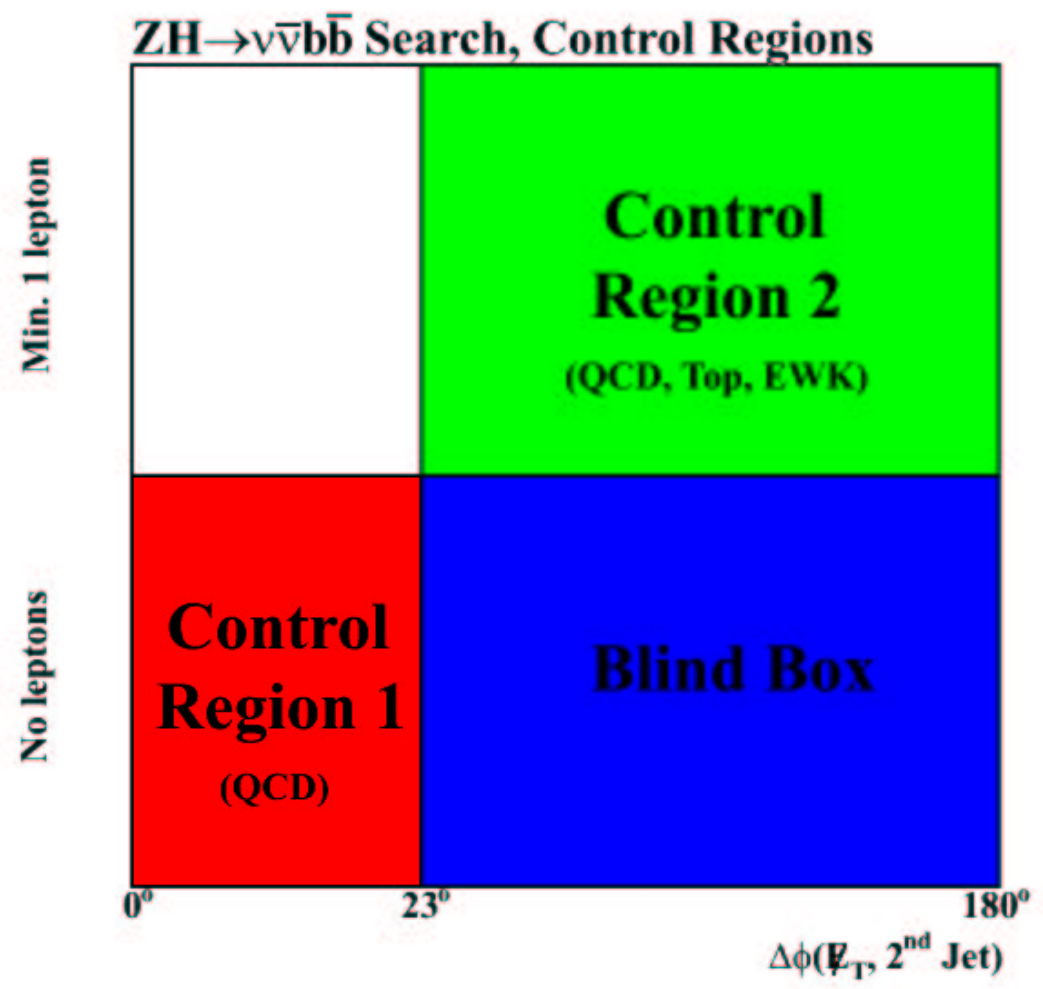

Fig. 6.1. The definition of the Control Regions 


\subsection{QCD Normalization}

The QCD heavy flavor simulation is normalized to the LO cross-section provided by Pytha scaled with a k-factor. We use this method to obtain a normalization factor that does not scale with the Monte Carlo statistics and can be compared with cross-sections used in similar analyses.

We choose a k-factor of 1.4 based on preliminary studies. However, there is no strong motivation to use this number and no justifiable systematic uncertainty to assign. We can compute an absolute estimate for every other background using the formula

$$
N_{\text {pred }}=\epsilon \cdot k \cdot \sigma_{L O} \cdot \mathcal{L}=\epsilon \cdot \sigma_{N L O} \cdot \mathcal{L}
$$

where $\mathcal{L}$ is the luminosity of the data, $\sigma_{N L O}$ is the higher order cross-section of the background process, and $\epsilon$ is the acceptance of the selection cuts. Thus it is possible to calculate the number of QCD events by subtracting the non-QCD background prediction from data and scaling the QCD to the difference.

This method provides a normalization that is only applicable in the region where the calculation was performed. In order to obtain a single normalization that is applicable in every region defined earlier, we perform the calculation with two different sets of cuts and get the normalization factor for the QCD by computing the error weighted average of the two scale factors. The errors are derived from the statistical errors in the data and in both QCD and non-QCD background simulations, and the systematic uncertainties in the non-QCD simulation (but not in the QCD to avoid double-counting). If the average is not consistent with the two measurements within their uncertainties then it is because one or both the uncertainties are underestimated. If the disagreement is not large, we scale the uncertainty of the average until it agrees with both measurements. 
Since the QCD is already approximately normalized, we only need to correct the $\mathrm{k}$-factor. The corrected $\mathrm{k}$-factor, $k_{\text {corr }}$, is computed by the following formula

$$
k_{\text {corr }}=k \times \frac{\left(N_{\text {Data }}-N_{\text {Data }}^{\text {mistag }}\right)-\left(N_{N o n-Q C D}-N_{\text {Non-QCD }}^{\text {mistag }}\right)}{N_{Q C D}-N_{Q C D}^{\text {mistag }}}
$$

which can be expressed as

$$
k_{\text {corr }}=k \times \frac{N_{\text {Data }}-N_{N o n-Q C D}-N^{\text {mistag }}-N_{Q C D}^{\text {mistag }}}{N_{Q C D}-N_{Q C D}^{\text {mistag }}}
$$

from

$$
N^{\text {mistag }}=N_{\text {Data }}^{\text {mistag }}-N_{Q C D}^{\text {mistag }}-N_{N o n-Q C D}^{\text {mistag }}
$$

where $N^{\text {mistag }}$ is the total mistag prediction and $N_{Q C D}^{\text {mistag }}$ is the mistag matrix prediction for the QCD Monte Carlo events (see last paragraph in Section 5.2.4). The average $k_{\text {corr }}$ is then calculated using the propagated error on each $k_{c o r r}$. The propagated error, among others, contains the uncertainty of the mistag estimation. Since the total mistag prediction is the difference between the mistag matrix applied to the data, $N_{\text {Data }}^{\text {mistag }}$, and to the simulations which include the QCD; therefore, the uncertainty on the number of mistags depends on the number of QCD events that we are computing. We use only one iteration in the calculation, since the QCD normalization with $\mathrm{k}=1.4$ is already close to the final value.

Normalized in such a way, the QCD simulation does not lose its predictive power, because the shape of distributions does not change due to scaling. In order to minimize the possibility of any bias in the rate prediction, we choose the two regions such that they do not overlap with the signal region. The first normalization factor is measured when $\varphi\left(2^{n d}\right.$ jet, $\left.\mathbb{E}_{T}\right)<0.4$, the second is calculated when $\not_{T}$ is not aligned with either of the leading jets $\left(\varphi\left(2^{n d} j e t, \notin_{T}\right)>0.4\right.$ and $\left.\varphi\left(1^{s t} j e t, \notin_{T}\right)>0.4\right)$, and $\not_{T}<70 \mathrm{GeV}$. The cut on $\mathbb{E}_{T}$ is required in order to discard signal-like events. The threshold was chosen based on a preceding Monte Carlo driven signal optimization in which the QCD was normalized with the uncorrected k-factor.

The correct normalization can depend on the number of tags found in the event. Double tag QCD events contain mostly b-quark pairs, but single tag events still have 
large contributions from mistags, charm, and b-quark pairs from gluon splittings that are not resolved in two jets. If Pythia gave a perfect description of the heavy flavor fraction and event kinematics, the QCD background could be just normalized with a single scale factor. Since we expect differences between single and double tags, the normalization is performed separately. The relative difference between the scale factors found in single and double tagged events is $13 \%$.

Table 6.1 shows the input numbers for Equation (6.3) in the single tag regions. The uncertainty on the average scale factor was increased from the estimated 0.02 to 0.04 in order to make it consistent with the scale factors found in the two kinematic regions. This is not a significant change in the uncertainty with respect to the size of other uncertainties, but it is necessary since we do not know if the reason for the difference is accounted for by any other systematic uncertainties ${ }^{1}$. The double tag normalization factors computed from Table 6.2 are both consistent with the weighted average within the estimated error.

It is expected that the jet energy scale (JES) would introduce an uncertainty in $\varphi\left(1^{s t} j e t, \notin_{T}\right)$ and $\varphi\left(2^{n d} j e t, \mathscr{H}_{T}\right)$ since $\phi_{\not_{T}}$ is recomputed after correcting $\not_{T}$ for the clustered energy. The $\varphi\left(j e t, \not_{T}\right)<0.4$ condition selects events where the $\mathscr{E}_{T}$ is due to jet mismeasurements, therefore, where the generic jet-correction is most likely to fail in correcting heavy flavor jets. The average scale factors were recomputed after propagating the JES uncertainty of the QCD prediction into the uncertainties of the individual measurements and was found to be consistent with each measurements. We do not use this result, otherwise the JES uncertainty would be partly doublecounted when it is included in the limit calculation. It is more appropriate to deal with the JES uncertainty after the final cuts, where all the JES related effects are evaluated.

${ }^{1}$ Different heavy flavor production modes have slightly different kinematic distributions. We may introduce a bias to the normalization by dividing the QCD events into two kinematic regions. The fact that we do not need to change the uncertainty much implies that this is not a significant problem. 
Table 6.1

Events used in adjusting the QCD normalization in the exclusive single tag sample.

\begin{tabular}{|c|c|c|}
\hline Process & $\varphi\left(2^{\text {nd }} j e t, \notin_{T}\right)<0.4$ & $\begin{array}{c}\varphi\left(1^{\text {st }} j e t, \notin_{T}\right)>0.4 \text { and } \\
\varphi\left(2^{n d} j e t, \notin_{T}\right)>0.4 \text { and } \\
\mathbb{E}_{T}<70 \mathrm{GeV}\end{array}$ \\
\hline QCD multi-jet & $16171.8 \pm 74.8$ & $1166.5 \pm 20.2$ \\
\hline TOP & $9.7 \pm 0.24 \pm 1.4$ & $57.8 \pm 0.6 \pm 8.9$ \\
\hline Di-boson & $0.99 \pm 0.11 \pm 0.16$ & $13.5 \pm 0.4 \pm 2.6$ \\
\hline $\mathrm{W}+$ h.f. & $20.0 \pm 1.2 \pm 9.0$ & $142.8 \pm 3.2 \pm 73.3$ \\
\hline $\mathrm{Z}+$ h.f. & $6.1 \pm 0.5 \pm 2.5$ & $41.6 \pm 1.3 \pm 17.9$ \\
\hline Mistag $\mathrm{N}^{\text {mistag }}$ & $3490.4 \pm 48.2 \pm 94.9$ & $227.2 \pm 5.4 \pm 5.3$ \\
\hline$\left(\begin{array}{l}N_{Q C D}^{\text {mistag }} \\
)\end{array}\right.$ & $(1781.6 \pm 24.1)$ & $(129.3 \pm 1.8)$ \\
\hline$\left(N_{\text {Data }}^{\text {mistag }}\right)$ & $(5276.6 \pm 32.2)$ & $(376.4 \pm 4.4)$ \\
\hline Data & 18746 & 1406 \\
\hline$k_{\text {corr }}$ & $1.31 \pm 0.02$ & $1.07 \pm 0.13$ \\
\hline Average scale factor & \multicolumn{2}{|c|}{$1.30 \pm 0.04$} \\
\hline
\end{tabular}

\subsection{Control Region 1}

Control Region 1 was constructed such that it dominantly contains QCD multi-jet events, it shows that the average normalization factor provides consistent prediction for this background. The region is split into exclusive single tag and double tag samples. The double tag sample contains very little mistag around $6 \%$ which is less than the total systematic uncertainty. Here we have a good handle on the shape and rate prediction of the QCD Monte Carlo. The single tag region contains much more, almost $20 \%$ mistag. The total background prediction is consistent with the data. Table 6.3 lists events in the single and the double tags. 
Table 6.2

Events used in adjusting the QCD normalization in the double tag sample.

\begin{tabular}{|c|c|c|}
\hline Process & $\varphi\left(2^{\text {nd }}\right.$ jet,$\left.\notin_{T}\right)<0.4$ & $\begin{array}{c}\varphi\left(1^{\text {st }} \text { jet, } \notin_{T}\right)>0.4 \text { and } \\
\varphi\left(2^{\text {nd }} \text { jet, } \notin_{T}\right)>0.4 \text { and } \\
\notin_{T}<70 \mathrm{GeV}\end{array}$ \\
\hline QCD multi-jet & $1156.9 \pm 19.5$ & $30.8 \pm 3.21$ \\
\hline TOP & $2.26 \pm 0.11 \pm 0.37$ & $12.29 \pm 0.26 \pm 2.08$ \\
\hline Di-boson & $0.07 \pm 0.02 \pm 0.02$ & $1.44 \pm 0.09 \pm 0.27$ \\
\hline $\mathrm{W}+$ h.f. & $0.43 \pm 0.16 \pm 0.2$ & $4.93 \pm 0.57 \pm 2.40$ \\
\hline Z + h.f. & $0.37 \pm 0.09 \pm 0.15$ & $2.59 \pm 0.24 \pm 1.17$ \\
\hline Mistag $\mathrm{N}^{\text {mistag }}$ & $56.0 \pm 9.3 \pm 9.8$ & $4.56 \pm 1.19 \pm 0.30$ \\
\hline$\left(\begin{array}{c}N_{Q C D}^{\text {mistag }} \\
)\end{array}\right)$ & $(268.3 \pm 6.8)$ & $(11.4 \pm 0.5)$ \\
\hline$\left(N_{\text {Data }}^{\text {mistag }}\right)$ & $(324.7 \pm 6.1)$ & $15.7 \pm 0.9$ \\
\hline Data & 1260 & 54 \\
\hline$k_{\text {corr }}$ & $1.47 \pm 0.07$ & $1.21 \pm 0.67$ \\
\hline Average scale factor & \multicolumn{2}{|c|}{$1.47 \pm 0.07$} \\
\hline
\end{tabular}

Kinematic distributions in Figure 6.4 indicate that the mistag estimation method works well, and the total shape is also well-predicted. In the other regions the background is more complex and gives only indirect information about mistags.

Control Region 1 (CR-1) has a slightly different composition of heavy flavor production modes than the Signal Region due to the cut on $\varphi\left(2^{\text {nd }} j e t, \notin_{T}\right)$. When the b-quarks are back-to-back (e.g. b-quark produced in the hard scattering), the $\mathbb{E}_{T}$ originates from mismeasuring one or more jets. The $E_{T}$ of the leading jet is large because in the back-to-back topology the mismeasurements of the jets balance each other to some extent (mismeasurement generally means underestimation). By requiring the second jet to pass a minimum threshold, in our case $25 \mathrm{GeV}$, and the event to have large $\not_{T}$ simultaneously, we bias the leading jets toward high $E_{T}$. The large $\mathbb{E}_{T}$ 

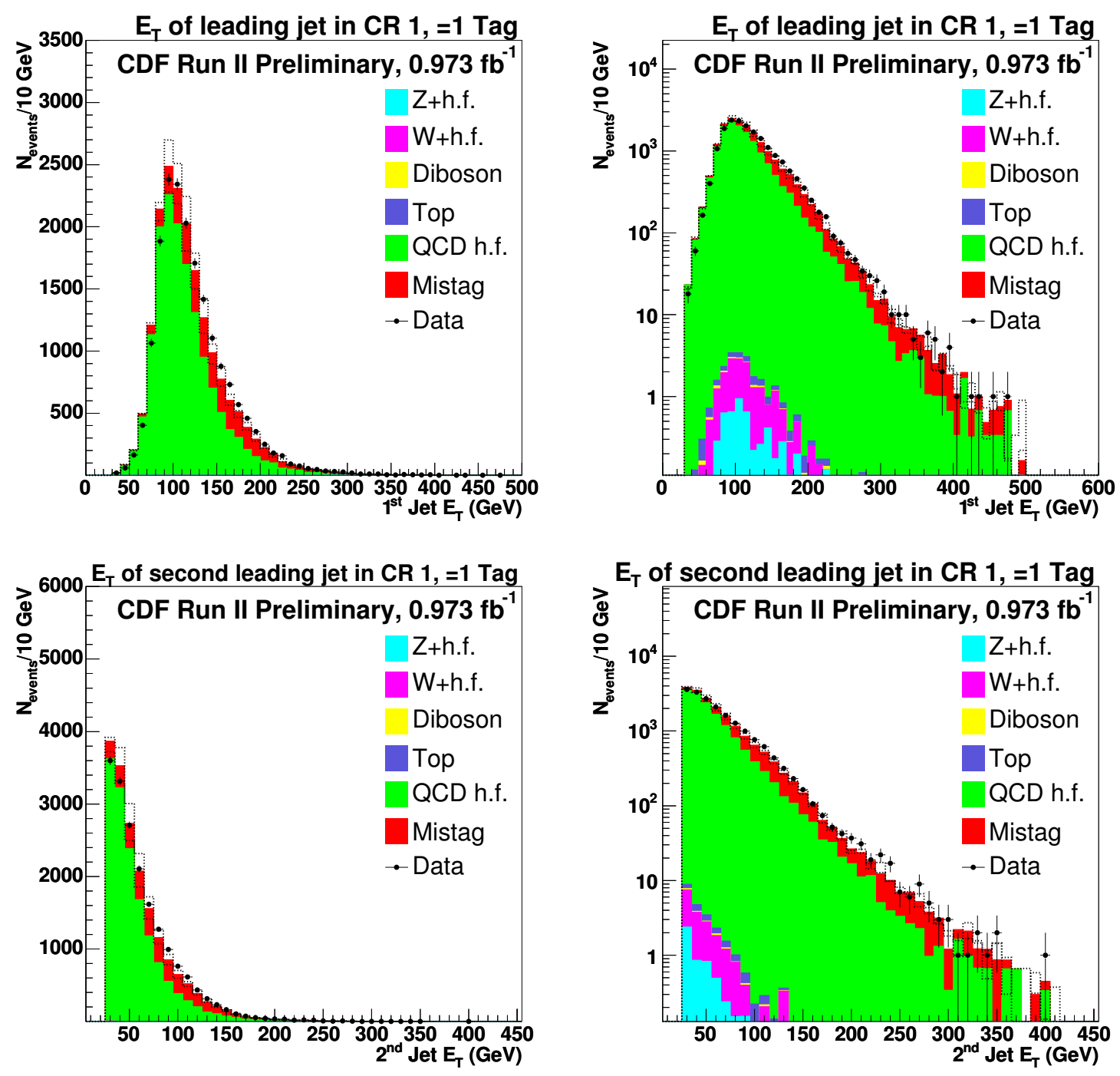

Fig. 6.2. Control Region 1, single tag: $1^{\text {st }}$ jet $E_{T}$ and $2^{\text {nd }}$ jet $E_{T}$ on linear and logarithmic scales 

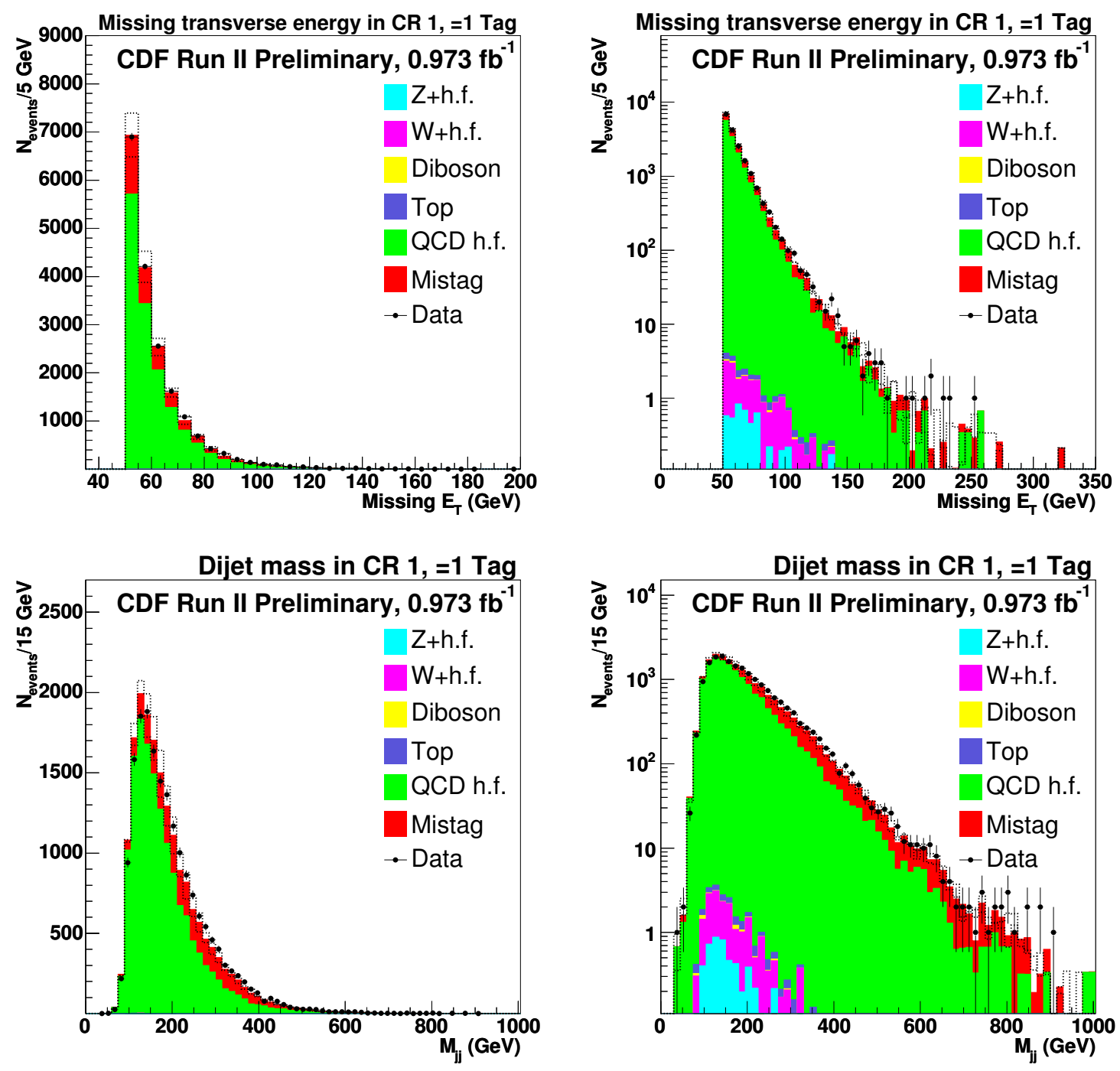

Fig. 6.3. Control Region 1, single tag: $\mathbb{E}_{T}$ and dijet mass on linear and logarithmic scales 
Table 6.3

Number of expected and observed events in Control Region 1.

\begin{tabular}{|l|c|c|}
\hline Process & Single Tag & Double Tag \\
\hline \hline QCD multi-jet & $14868 \pm 1820$ & $1175 \pm 179$ \\
TOP & $4.0 \pm 0.6$ & $0.8 \pm 0.2$ \\
Di-boson & $0.7 \pm 0.1$ & $0.04 \pm 0.02$ \\
W + h.f. & $14.0 \pm 6.7$ & $0.3 \pm 0.2$ \\
Z + h.f. & $5.3 \pm 2.2$ & $0.3 \pm 0.2$ \\
Mistag & $3460 \pm 433$ & $73 \pm 14$ \\
\hline \hline Expected & $18352 \pm 2031$ & $1249 \pm 203$ \\
\hline Observed & 18588 & 1251 \\
\hline
\end{tabular}

requirement and the cut on $\varphi\left(2^{n d} j e t, \mathbb{E}_{T}\right)$ are the reasons why the prediction of this region is very sensitive to the jet energy scale. Figure 6.2 indicates an approximately one sigma (3\%) systematic shift between the jet energy distributions of the simulation and the data.

In contrast, the Signal Region contains more events where the b-quarks are produced in gluon-splitting. In such cases, the b-jets are not back-to-back and their mismeasurements do not cancel each other; therefore, the leading jet in the Signal Region tends to be less energetic. The rate of these two processes determines the shape of the jet $E_{T}$ distributions in both Control and Signal Regions. The leading jet distribution in the Signal Region is a sum of an exponentially decreasing function and one with a peak at higher $E_{T}$. The uncertainty in $\varphi\left(2^{\text {nd }} j e t, \mathbb{E}_{T}\right)$ thus propagates into the jet $E_{T}$ distributions seen in the Signal Region. This is also covered by the JES uncertainty.

Figures 6.3, 6.4, and 6.5, 6.6, 6.7 show comparisons of various observables between the data and the simulation in the single and double tag samples. The dashed line shows the $\pm 1 \sigma$ variation in the JES. 

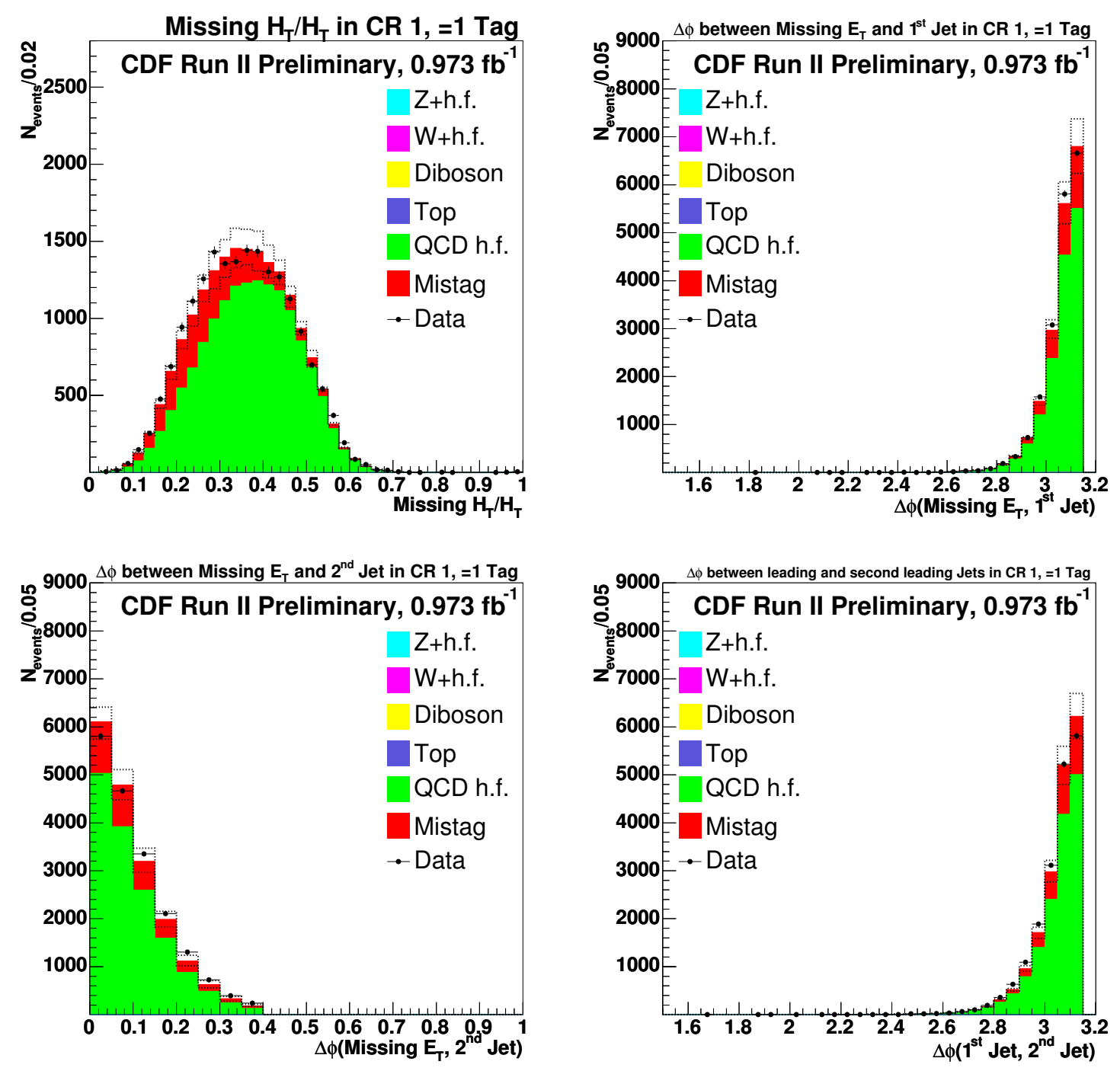

Fig. 6.4. Control Region 1, single tag: distribution of variables $\frac{\not f_{T}}{H_{T}}$, $\varphi\left(1^{\text {st }}\right.$ jet, $\left.\mathbb{E}_{T}\right), \varphi\left(2^{\text {nd }}\right.$ jet, $\left.\notin_{T}\right)$, and $\Delta \phi\left(1^{\text {st }}\right.$ jet, $2^{\text {nd }}$ jet $)$ 

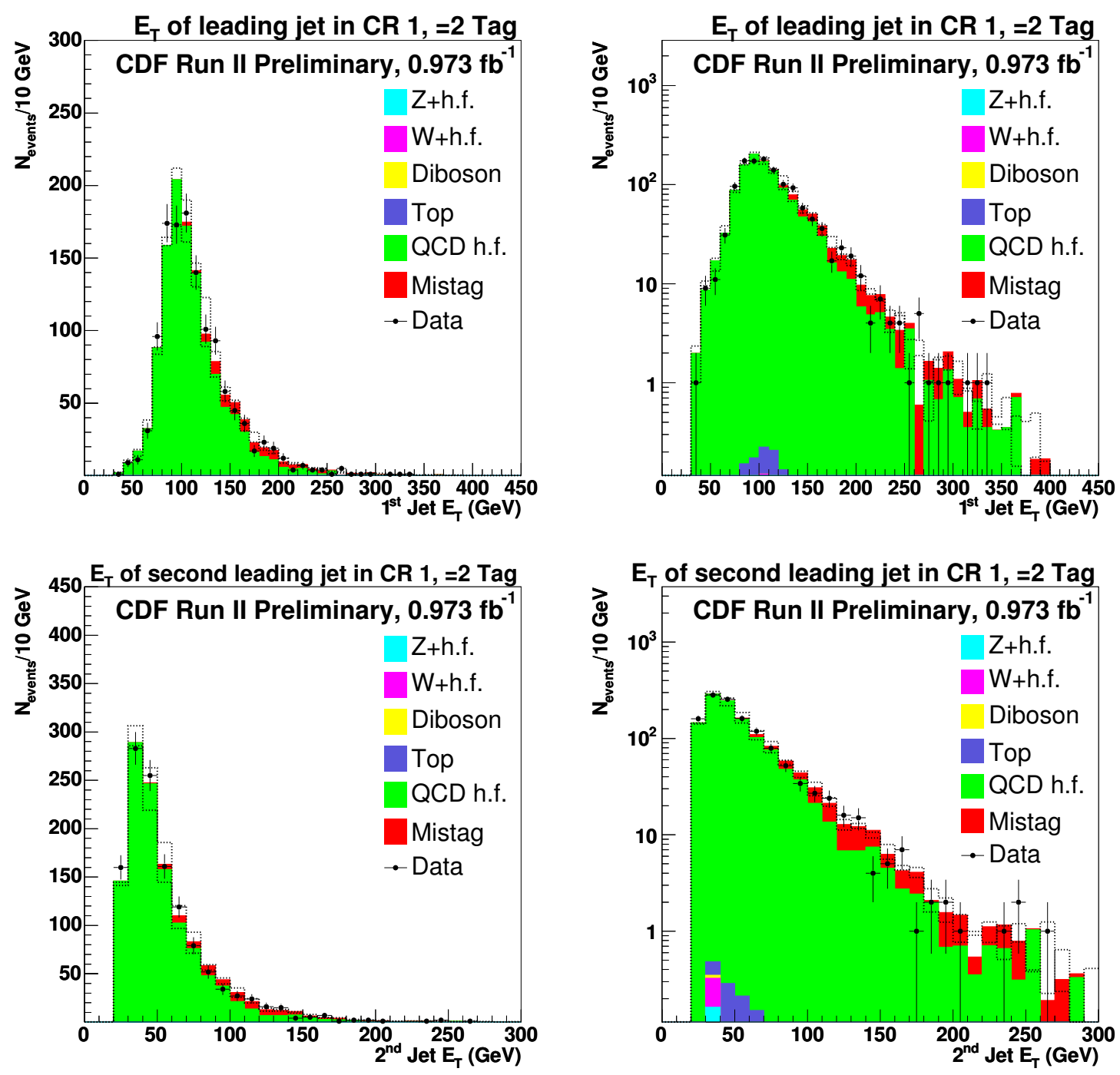

Fig. 6.5. Control Region 1, double tag: distribution of variables $1^{\text {st }}$ jet $E_{T}$ and $2^{\text {nd }}$ jet $E_{T}$ on linear and logarithmic scales 

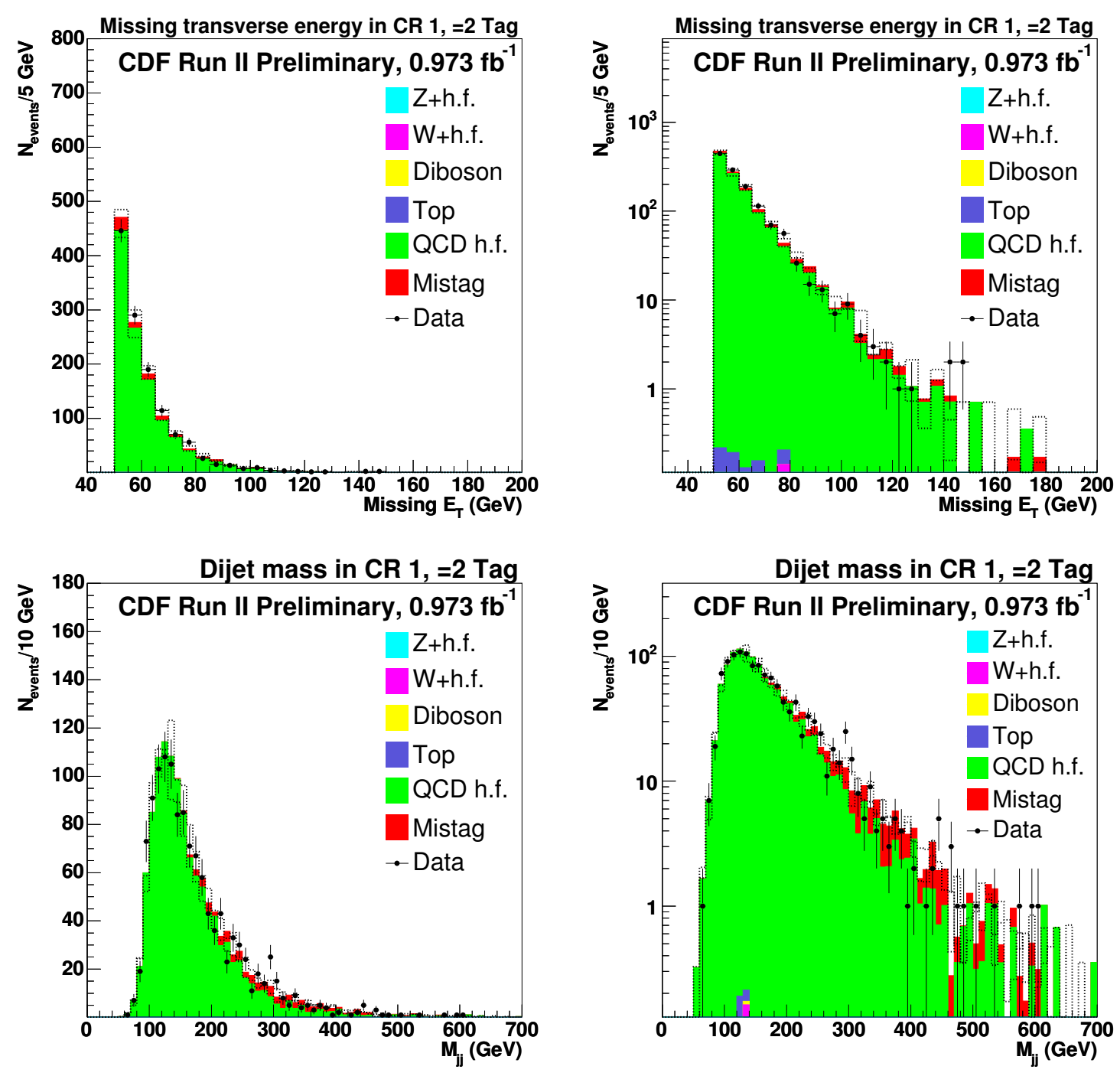

Fig. 6.6. Control Region 1, double tag: distribution of variables $\not_{T}$ and dijet mass on linear and logarithmic scales 

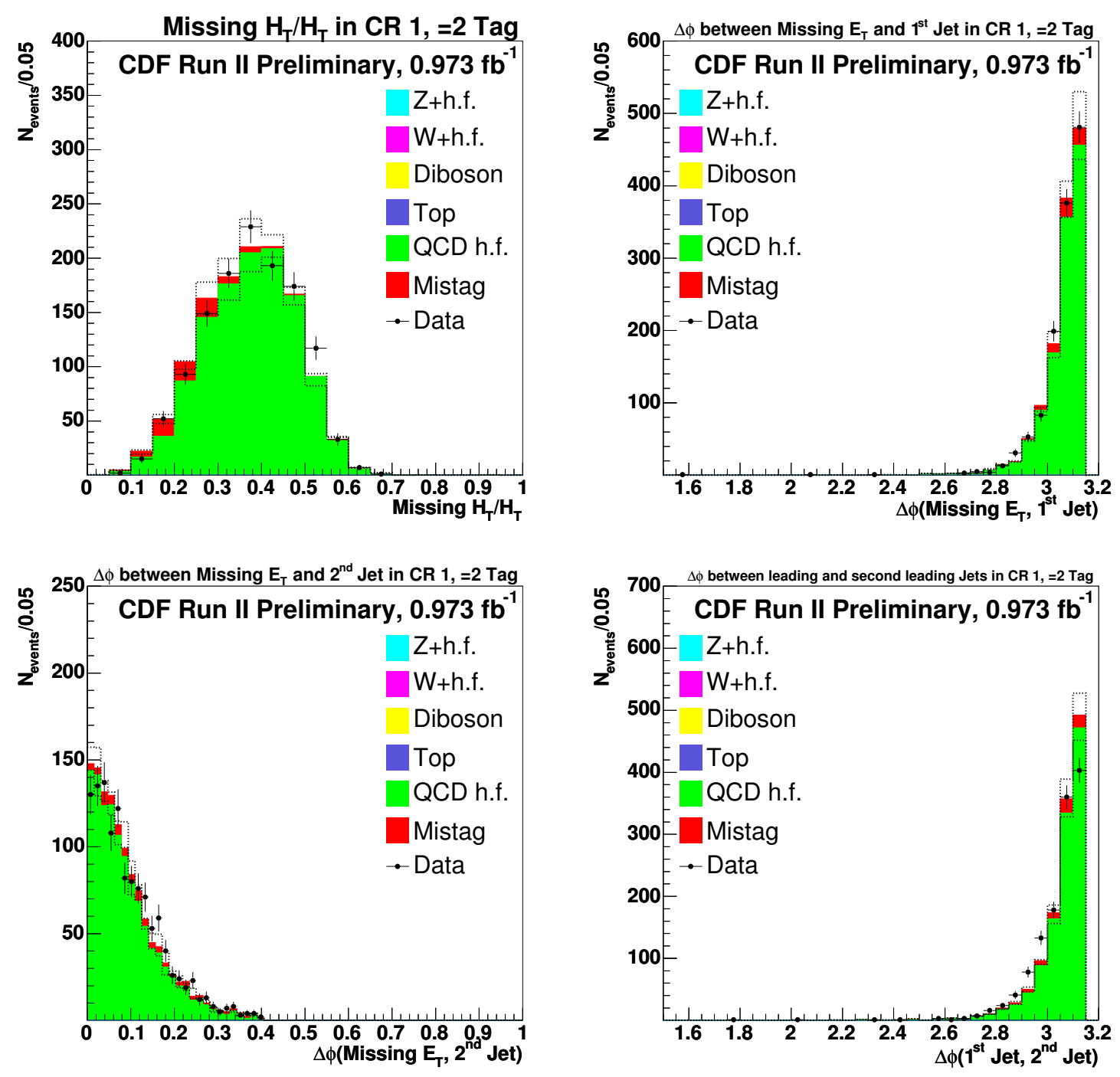

Fig. 6.7. Control Region 1, double tag: distribution of variables $\frac{\not f_{T}}{H_{T}}$, $\varphi\left(1^{\text {st }}\right.$ jet, $\left.\mathbb{E}_{T}\right), \varphi\left(2^{\text {nd }}\right.$ jet, $\left.\mathbb{E}_{T}\right), \Delta \phi\left(1^{\text {st }}\right.$ jet, $2^{\text {nd }}$ jet $)$ 


\subsection{Control Region 2}

This control region contains various SM processes. The double tag events provide an excellent check of the top simulation since they are dominated by top production. We observe a good agreement with the data as expected, since the top background was normalized to the average cross-section obtained from dedicated CDF analyses. The expected number of events are shown in Table 6.4 and the corresponding double tag plots in Figures 6.10 and 6.11. The dashed line shows the $\pm 1 \sigma$ variation in the JES. The single tag comparison plots are in Figures 6.8 and 6.9.

Table 6.4

Number of expected and observed events in Control Region 2.

\begin{tabular}{|l|c|c|}
\hline Process & Single Tag & Double Tag \\
\hline \hline QCD multi-jet & $61 \pm 25$ & $3.2 \pm 1.7$ \\
TOP & $98 \pm 14$ & $24.0 \pm 3.8$ \\
Di-boson & $11.5 \pm 2.1$ & $1.0 \pm 0.2$ \\
W + h.f. & $63 \pm 28$ & $3.9 \pm 1.8$ \\
Z + h.f. & $12.9 \pm 5.9$ & $0.5 \pm 0.4$ \\
Mistag & $87 \pm 11$ & $2.2 \pm 0.6$ \\
\hline \hline Expected & $333 \pm 51$ & $34.8 \pm 5.9$ \\
\hline Observed & 373 & 28 \\
\hline
\end{tabular}

The single tag sample with identified leptons consists of events with real isolated leptons, those with real non-isolated leptons incorrectly classified as isolated, and events with tracks that fake isolated leptons. $\mathrm{W} / \mathrm{Z}+$ jet processes are examples of the first case, and QCD h.f. production for the second and third.

The systematic uncertainty assigned to the lepton veto is expressed with respect to the number of events in the Signal Region. The systematic uncertainty on the real lepton identification with the loose lepton selection is typically at the percent level 

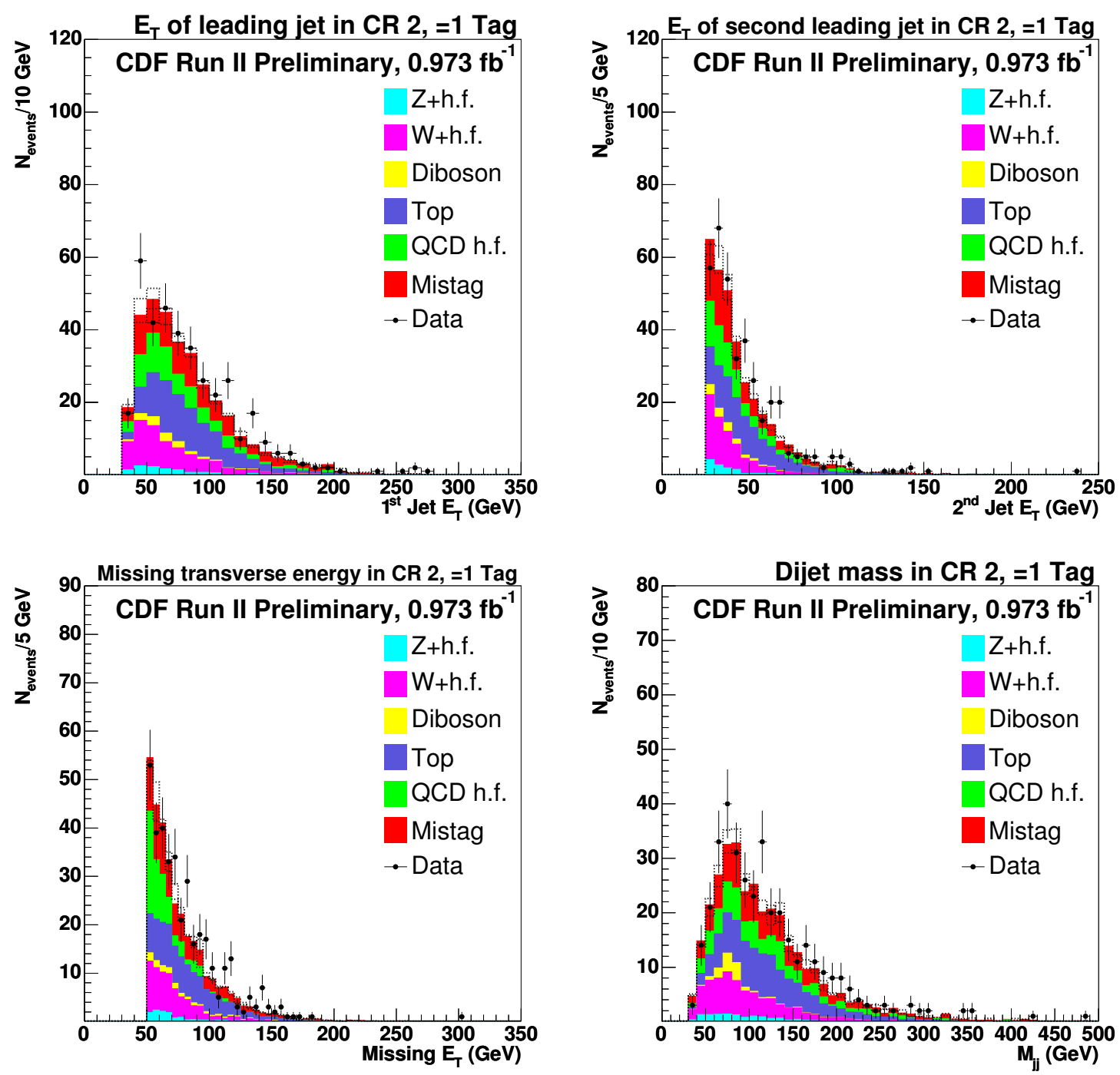

Fig. 6.8. Control Region 2, single tag: distribution of variables $1^{\text {st }}$ jet $E_{T}, 2^{\text {nd }}$ jet $E_{T}, E_{T}$, dijet mass 

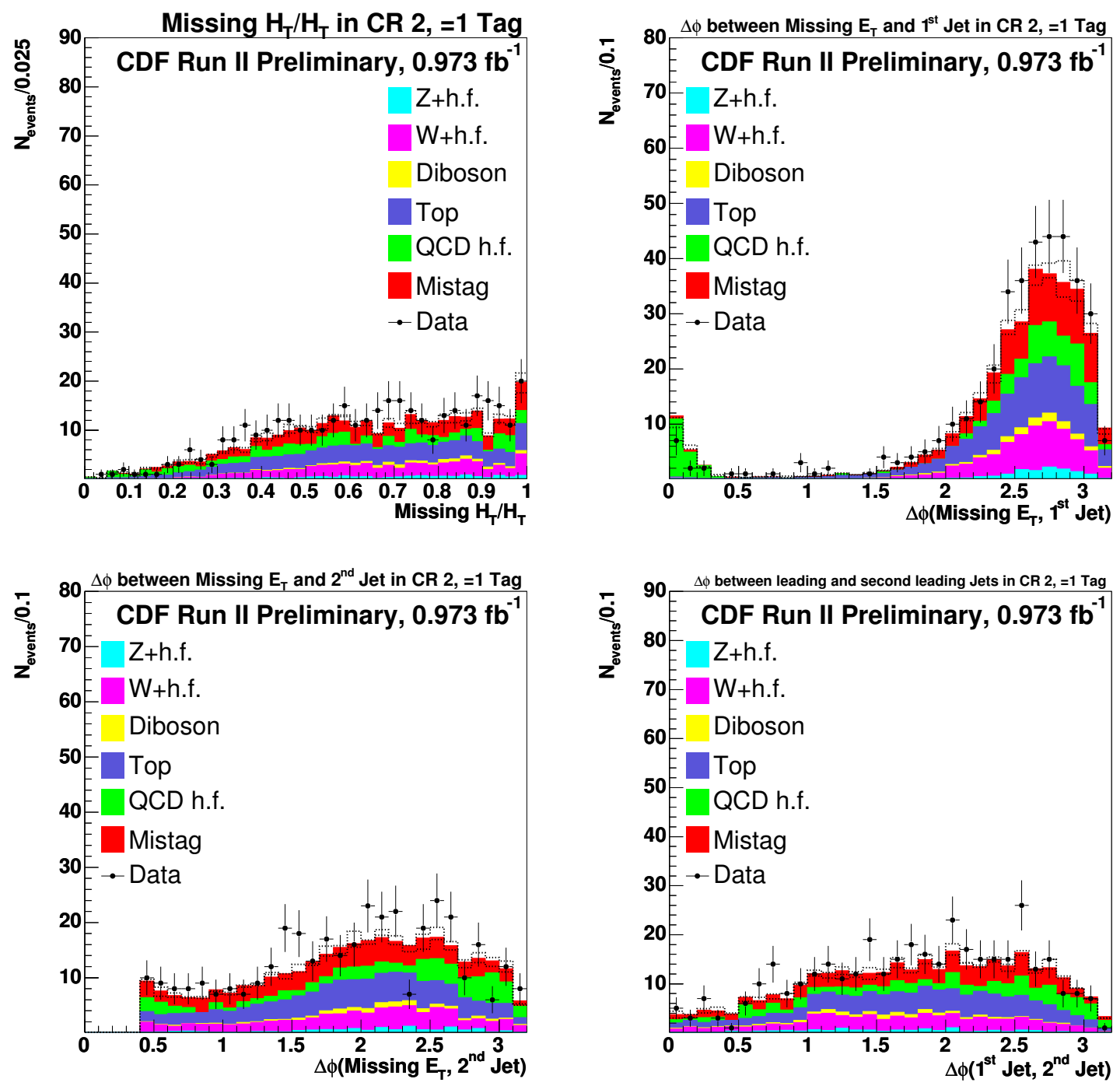

Fig. 6.9. Control Region 2, single tag: distribution of variables $\frac{\not f_{T}}{H_{T}}$, $\varphi\left(1^{\text {st }}\right.$ jet, $\left.\mathbb{E}_{T}\right), \varphi\left(2^{\text {nd }}\right.$ jet, $\left.\mathbb{E}_{T}\right), \Delta \phi\left(1^{\text {st }}\right.$ jet, $2^{\text {nd }}$ jet $)$ 
(see $[45,57])$ which is taken as the maximum difference of the lepton identification efficiency between Monte Carlo and data. In QCD events, the total fake lepton rate is also at the percent level but with a very large relative uncertainty. This uncertainty is estimated to be $2 \%$ by taking the difference between the fake lepton rates observed in a simulated jet sample and in an inclusive jet data. In order to simplify the treatment of the lepton veto uncertainty that is otherwise negligible with respect to other systematics, we assign an overall $2 \%$ to all simulated processes. Since Control Region 2 (CR-2) and the Signal Region are complementary subsets of the events where $\varphi\left(2^{\text {nd }}\right.$ jet, $\left.\mathbb{E}_{T}\right) \geq 0.4$, the $2 \%$ uncertainty in the Signal Region translates to a larger uncertainty in CR-2 depending on how the events are distributed between the two regions. For $\mathrm{W} / \mathrm{Z}+$ h.f. this means a 3\%-5\% uncertainty in Control Region 2 because there are more events without identified leptons. In our definition, the uncertainty on the fake lepton veto in QCD events is about $40 \%$ because $96 \%$ of the events do not have identified leptons.

The difference seen in Table 6.4 between the predicted and observed events is mostly due to the simulation of isolated lepton fakes in QCD. Its uncertainty may be underestimated in this region if the uncertainty on the fake isolated track rate depends on the topology of QCD heavy flavor events. This is supported by the fact that the fake lepton rate in QCD where $\varphi\left(2^{\text {nd }}\right.$ jet, $\left.\notin_{T}\right)<0.4$ is about half of the rate where $\varphi\left(2^{\text {nd }}\right.$ jet, $\left.\notin_{T}\right)>0.4$. The observed difference in this control region is already covered by the assigned systematics, any further study is unnecessary.

It is observed, nevertheless, that the QCD heavy flavor simulation does not reproduce the data perfectly for soft leptons and loose track isolation. We tighten the cut on the electron selection from $E_{T}>10 \mathrm{GeV}$ to $E_{T}>20 \mathrm{GeV}$, lowered the track isolation from 0.1 to 0.02 and required a minimum of $1 \mathrm{GeV}$ hadronic energy to be in the calorimeter cluster associated with the track. We get much better agreement between the simulation and the data after applying these tighter selections which eliminate a large fraction of QCD events. The number of expected events after these 

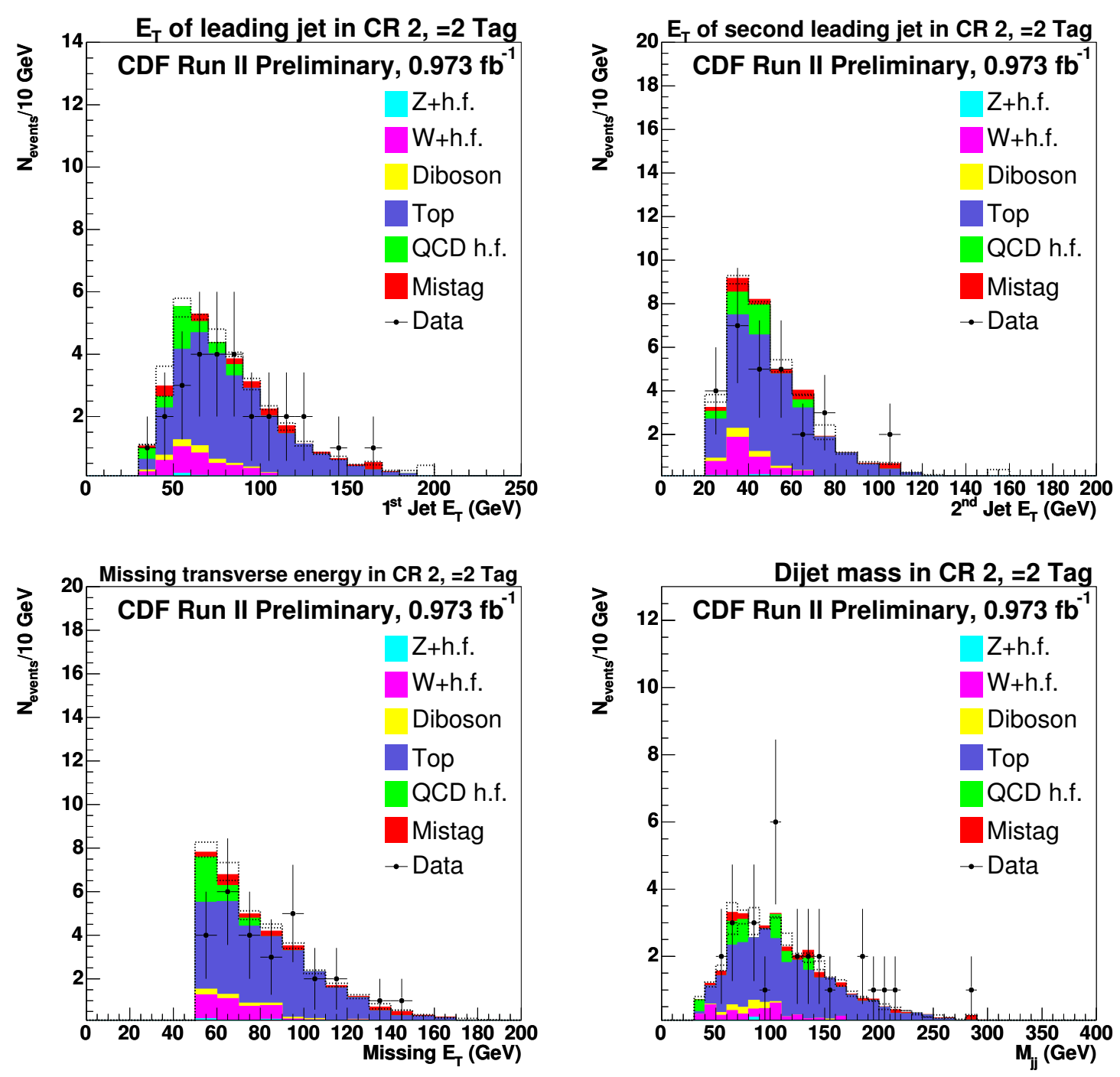

Fig. 6.10. Control Region 2, double tag: distribution of variables $1^{\text {st }}$ jet $E_{T}, 2^{\text {nd }}$ jet $E_{T}$, $\notin_{T}$, dijet mass 

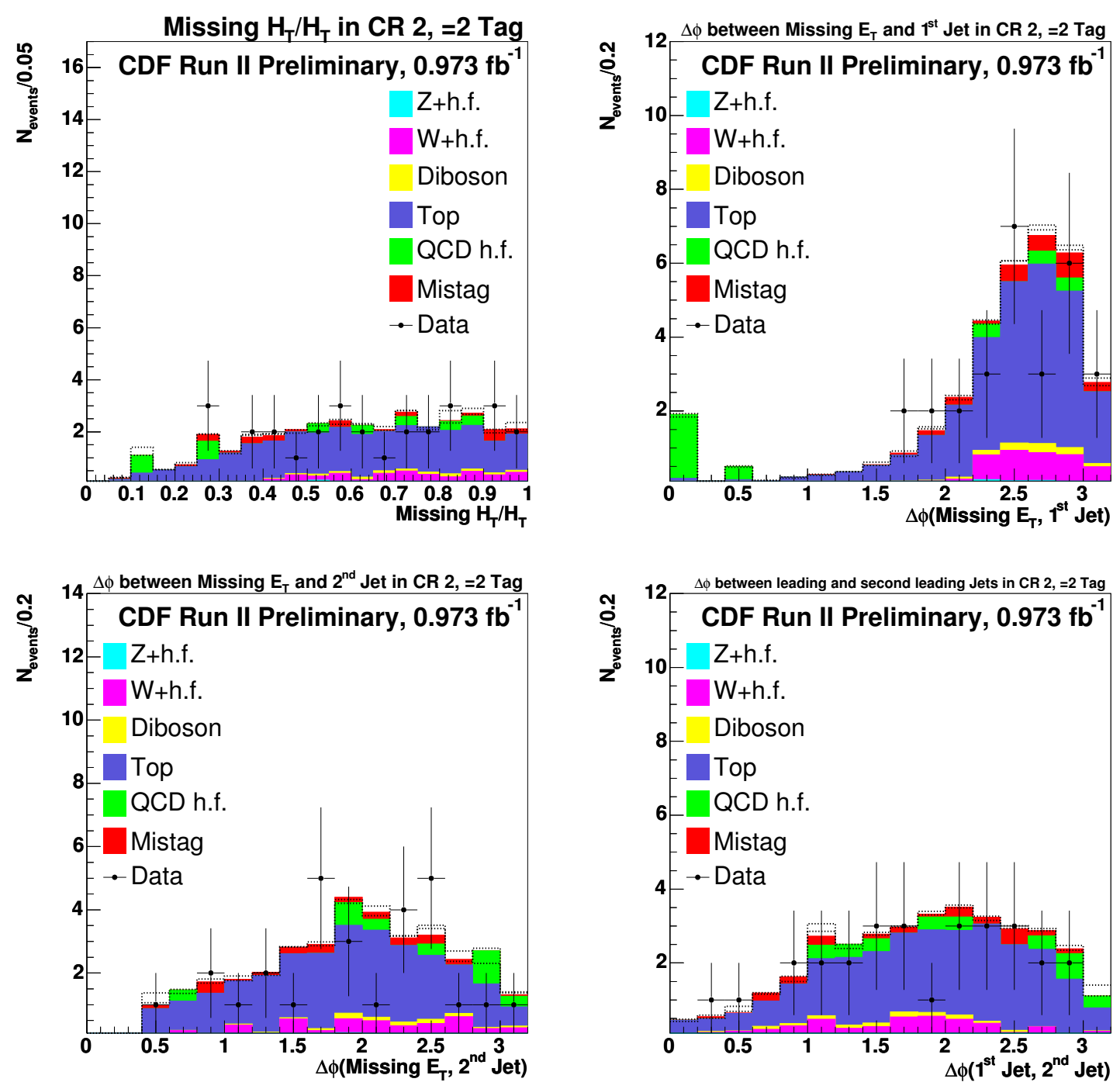

Fig. 6.11. Control Region 2, double tag: distribution of variables $\frac{\not f_{T}}{H_{T}}$, $\varphi\left(1^{\text {st }}\right.$ jet, $\left.\mathbb{E}_{T}\right), \varphi\left(2^{\text {nd }}\right.$ jet, $\left.\mathbb{E}_{T}\right), \Delta \phi\left(1^{\text {st }}\right.$ jet, $2^{\text {nd }}$ jet $)$ 
cuts are shown in Table 6.5 and the corresponding comparison plots in Figures 6.12 and 6.13. The dashed line shows the $\pm 1 \sigma$ variation in the JES.

Table 6.5

Expected and observed events in Control Region 2 in Exclusive Single Tag events after the tighter lepton selection.

\begin{tabular}{|l|c|}
\hline Process & Control Region 2 \\
\hline \hline QCD multi-jet & $30.2 \pm 11.5$ \\
TOP & $77 \pm 11$ \\
Di-boson & $8.5 \pm 1.5$ \\
W + h.f. & $46.6 \pm 20.4$ \\
Z + h.f. & $9.7 \pm 4.7$ \\
Mistag & $56.6 \pm 5.3$ \\
\hline \hline Expected & $229 \pm 33$ \\
\hline Observed & 230 \\
\hline
\end{tabular}

After the tight selection, a quarter of the events in this region is due to mistags. We can figure out the dominant physics processes in the mistags by noting that the QCD contribution is reduced to $13 \%$, the $33 \%$ top production does not give rise to a significant number of mistags in low jet-multiplicity having already two jets to tag, and the diboson production amounts to only 5\%. Therefore, the majority of the mistags must originate from $\mathrm{W} / \mathrm{Z}+$ light flavor jet production. Thus nearly half of the events in this region are $\mathrm{W} / \mathrm{Z}+$ jets. The top normalization has already been corroborated in the double tag sample; therefore, this control region confirms the prediction of the overall $\mathrm{W} / \mathrm{Z}$ background. 

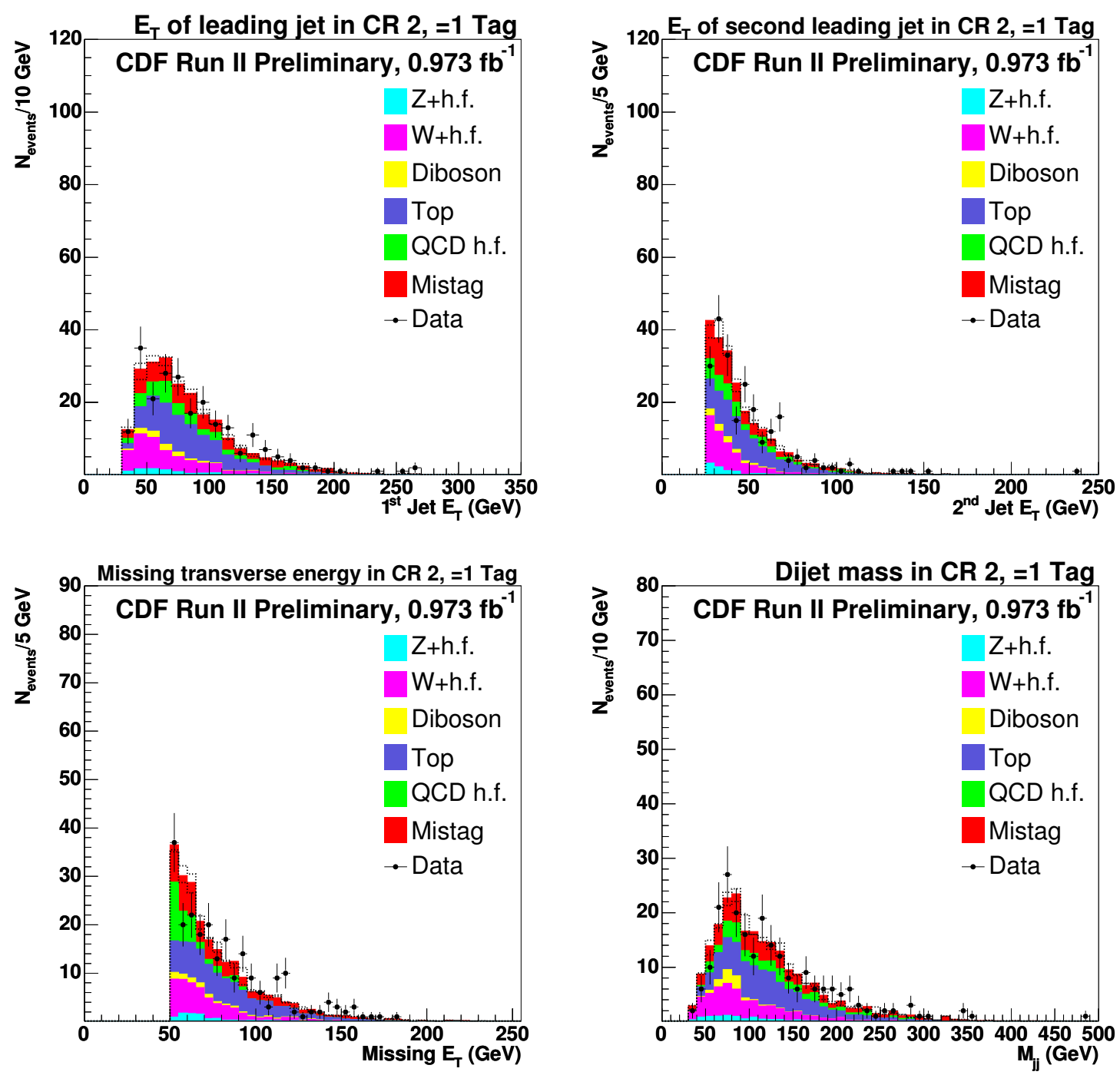

Fig. 6.12. Control Region 2, single tag, tight lepton selection: distribution of variables $1^{\text {st }}$ jet $E_{T}, 2^{\text {nd }}$ jet $E_{T}, \notin_{T}$, dijet mass 

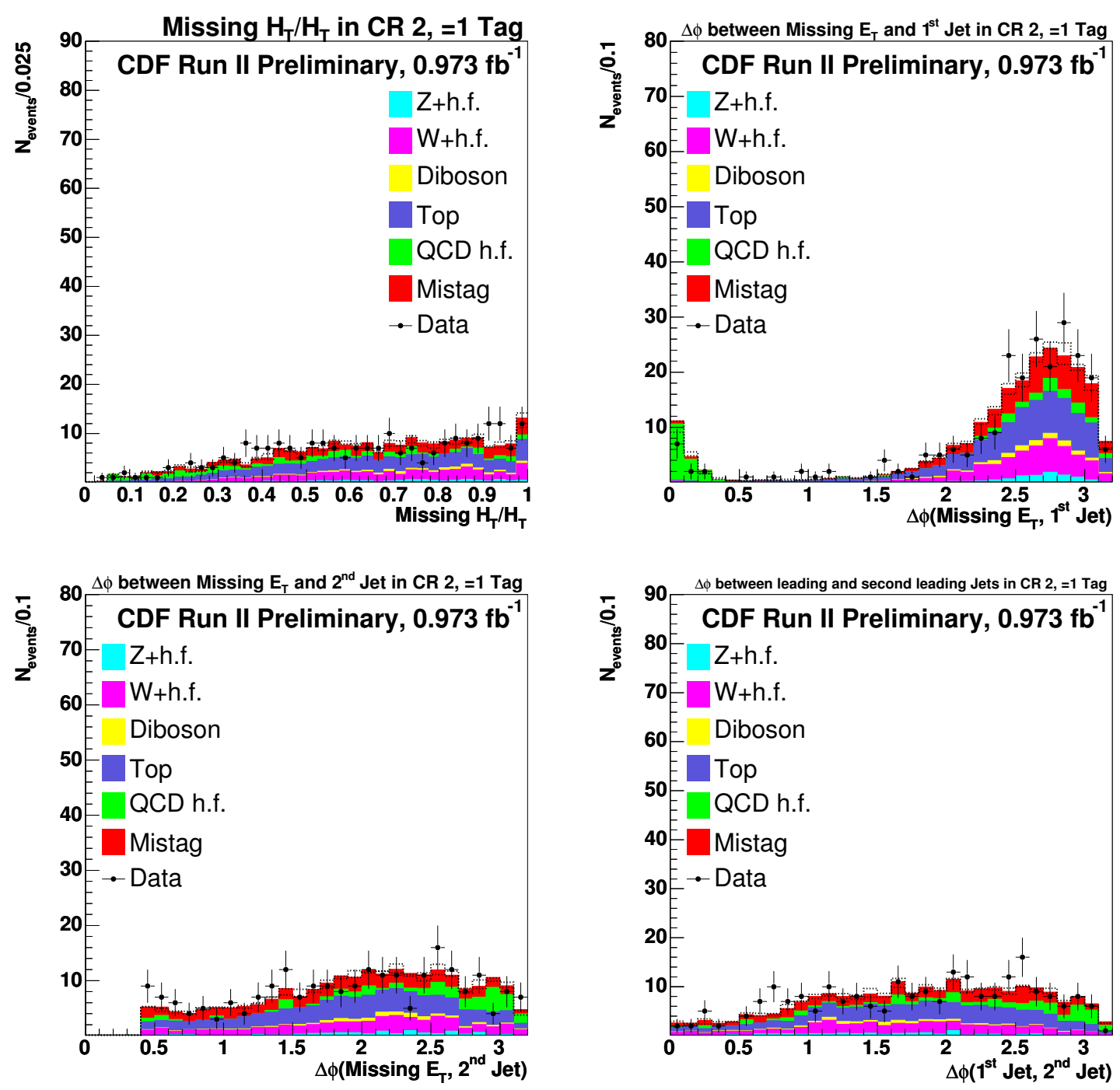

Fig. 6.13. Control Region 2, single tag, tight lepton selection: distribution of variables $\frac{\mu_{T}}{H_{T}}, \varphi\left(1^{\text {st }}\right.$ jet, $\left.\notin_{T}\right), \varphi\left(2^{\text {nd }}\right.$ jet, $\left.\notin_{T}\right), \Delta \phi\left(1^{s t} j e t, 2^{\text {nd }}\right.$ jet $)$ 


\subsection{Signal Region and Final Selection}

We demonstrate the selection criteria of our search in the signal region showing their effect on a $115 \mathrm{GeV}$ Higgs MC sample (see Section 5.1). The results are shown in Table 6.6. We look at the signal region after the final selection cuts are determined. The data - Monte Carlo comparison plots are shown in Figures 6.14, 6.15, 6.16 in the single tag and $6.17,6.18$ in the double tag events. The dashed lines are the $\pm 1 \sigma$ variations in the JES uncertainty. The number of expected and observed background events before applying the final selection are listed in Table 6.7.

A set of orthogonal cuts are used in the final optimization. These selection cuts maximize the $S / \sqrt{B}$ ratio for double tag events in the signal region after applying a $\pm 20 \mathrm{GeV}$ dijet mass window cut around the average dijet mass of the Higgs signal. The variables providing the best $S / \sqrt{B}$ were chosen from the available kinematic observables and applied sequentially. Developing more efficiency optimizations is a challenge, because the $\mathbb{E}_{T}$ cut represents by far the best separation power after requiring tags, cutting on the angles between jets and $\not_{T}$, and selecting a dijet masswindow. It is very difficult to accurately model the correlation between jet-related observables and $\notin_{T}$. An effective signal optimization also requires large statistics in the background simulation which is not available for all backgrounds using the current method. The final result, nevertheless, is mostly limited by data statistics in the double tags rather than the systematics; therefore, improvements will only be germane later when more data is processed.

The final selection is as follows:

- Azimuthal separation between leading jet and $\notin_{T}$ : $\left(1^{s t} j e t, \notin_{T}\right)>0.8$,

- $\frac{\not \mu_{T}}{H_{T}}>0.45$, where $H_{T}$ is the vectorial and $H_{T}$ is the scalar sum of the jet energies $^{2}$,

- $E_{T}$ of leading jet: $1^{\text {st }}$ jet $E_{T}>60 \mathrm{GeV}$,

${ }^{2}$ The magnitude of $\not H_{T}$ is the $\not_{T}$ corresponding to the clustered energy in the event. 

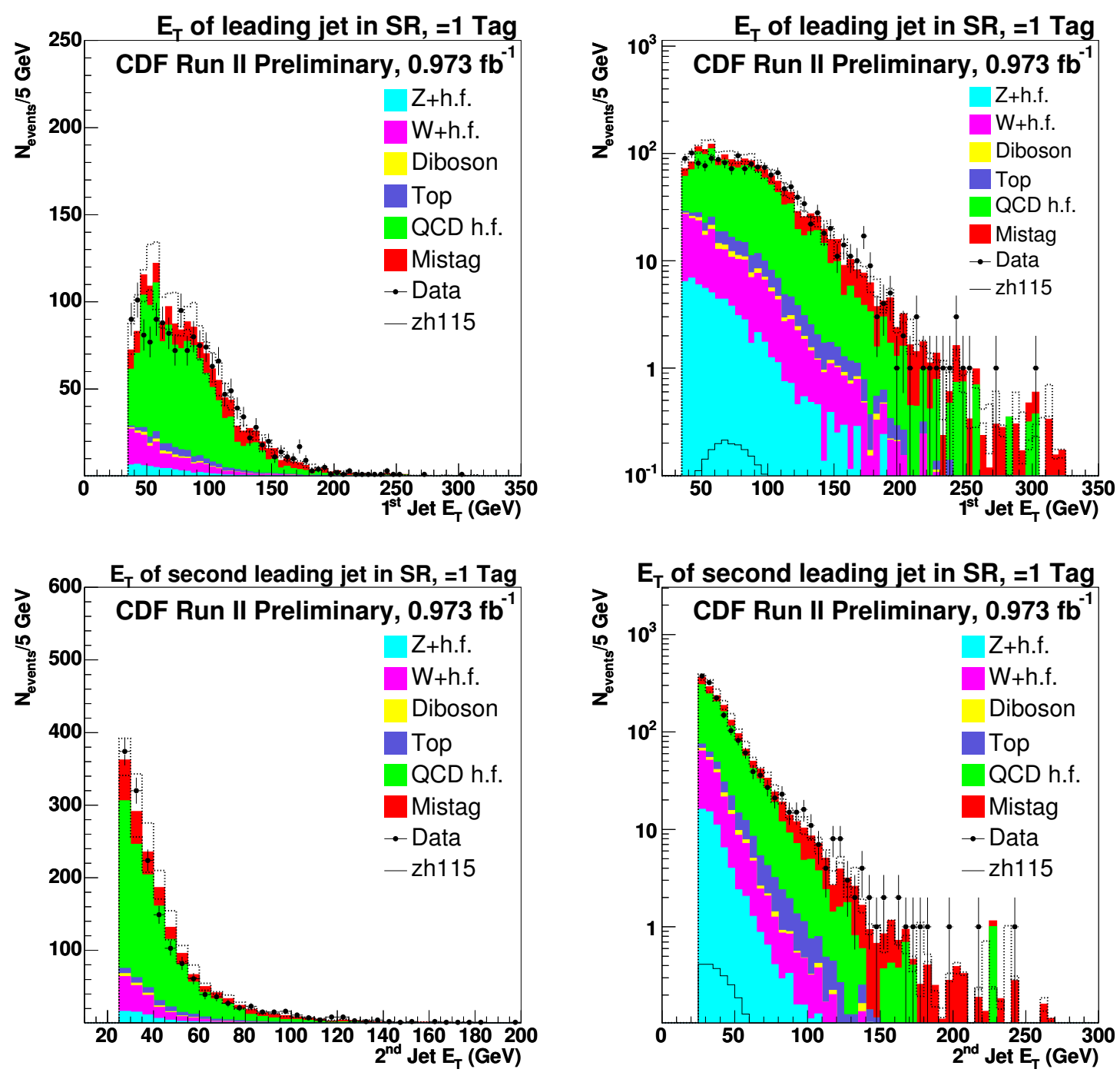

Fig. 6.14. Signal Region, single tag before final selection: distribution of variables $1^{\text {st }}$ jet $E_{T}$ and $2^{\text {nd }}$ jet $E_{T}$ on linear and logarithmic scales 

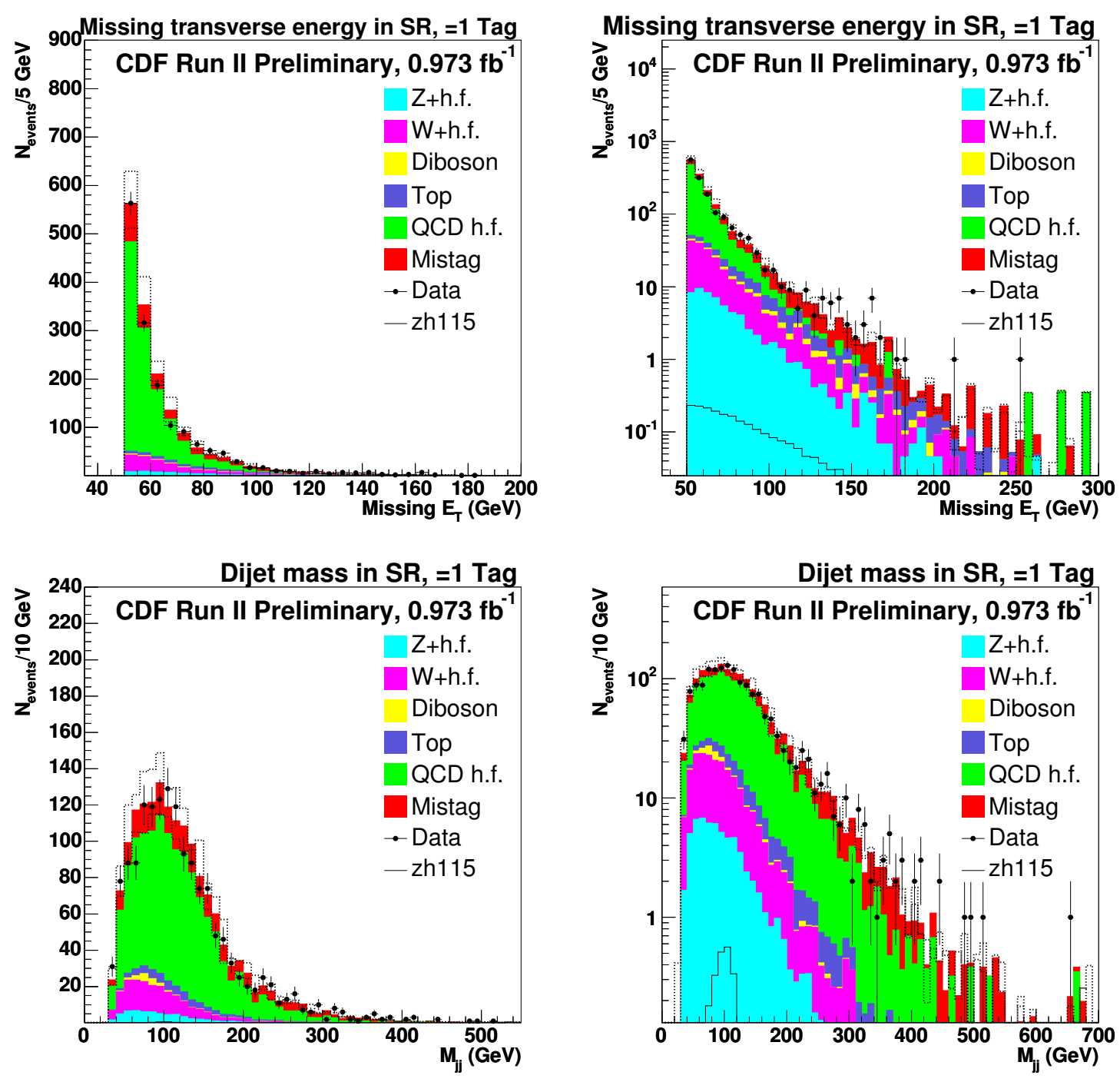

Fig. 6.15. Signal Region, single tag before final selection: distribution of variables $\not_{T}$ and dijet mass on linear and logarithmic scales 

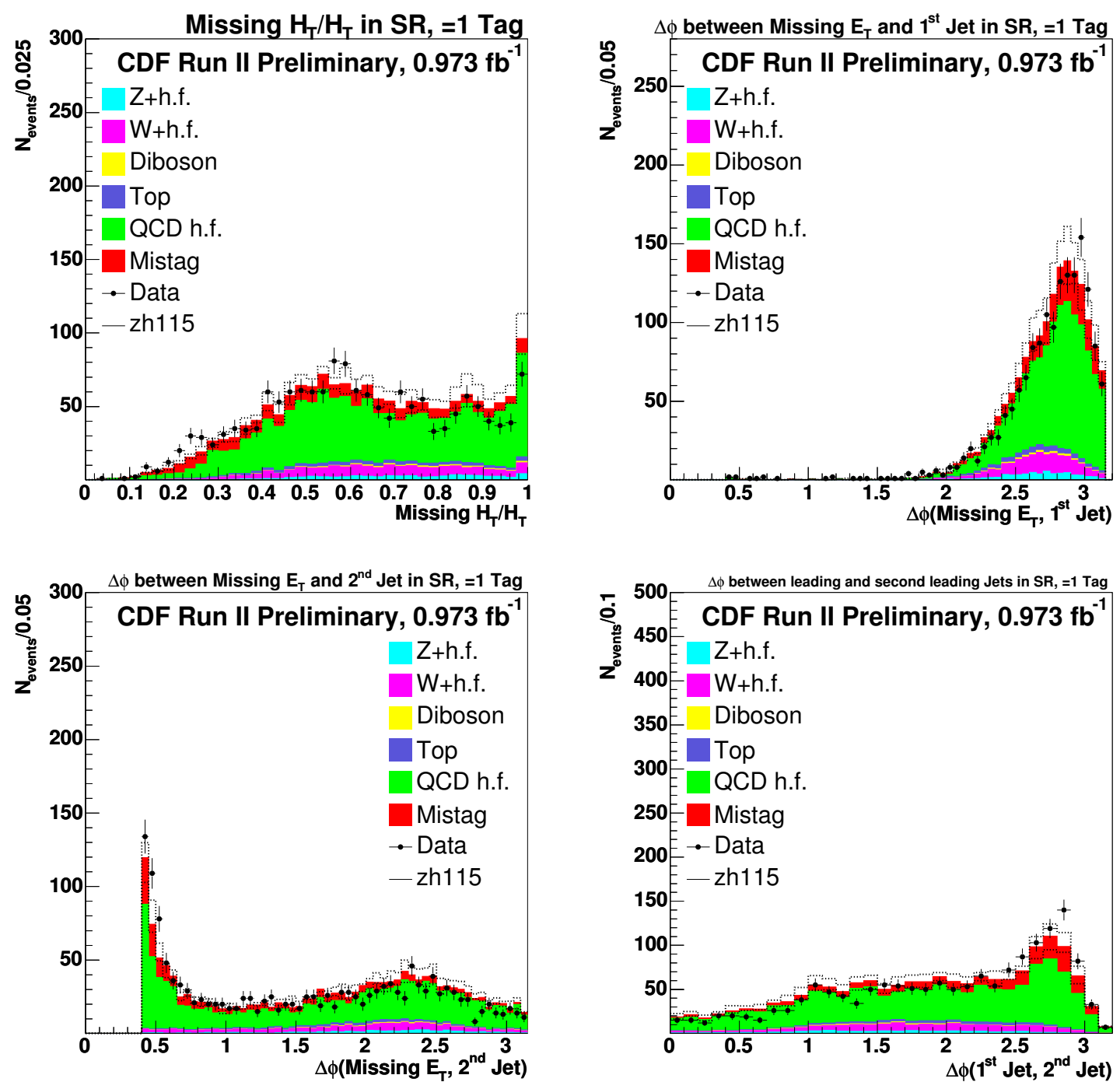

Fig. 6.16. Signal Region, single tag before final selection: distribution of variables $\frac{\not_{T}}{H_{T}}, \varphi\left(1^{s t} j e t, \notin_{T}\right), \varphi\left(2^{\text {nd }}\right.$ jet, $\left.\notin_{T}\right), \Delta \phi\left(1^{\text {st }} j e t, 2^{\text {nd }}\right.$ jet $)$ 

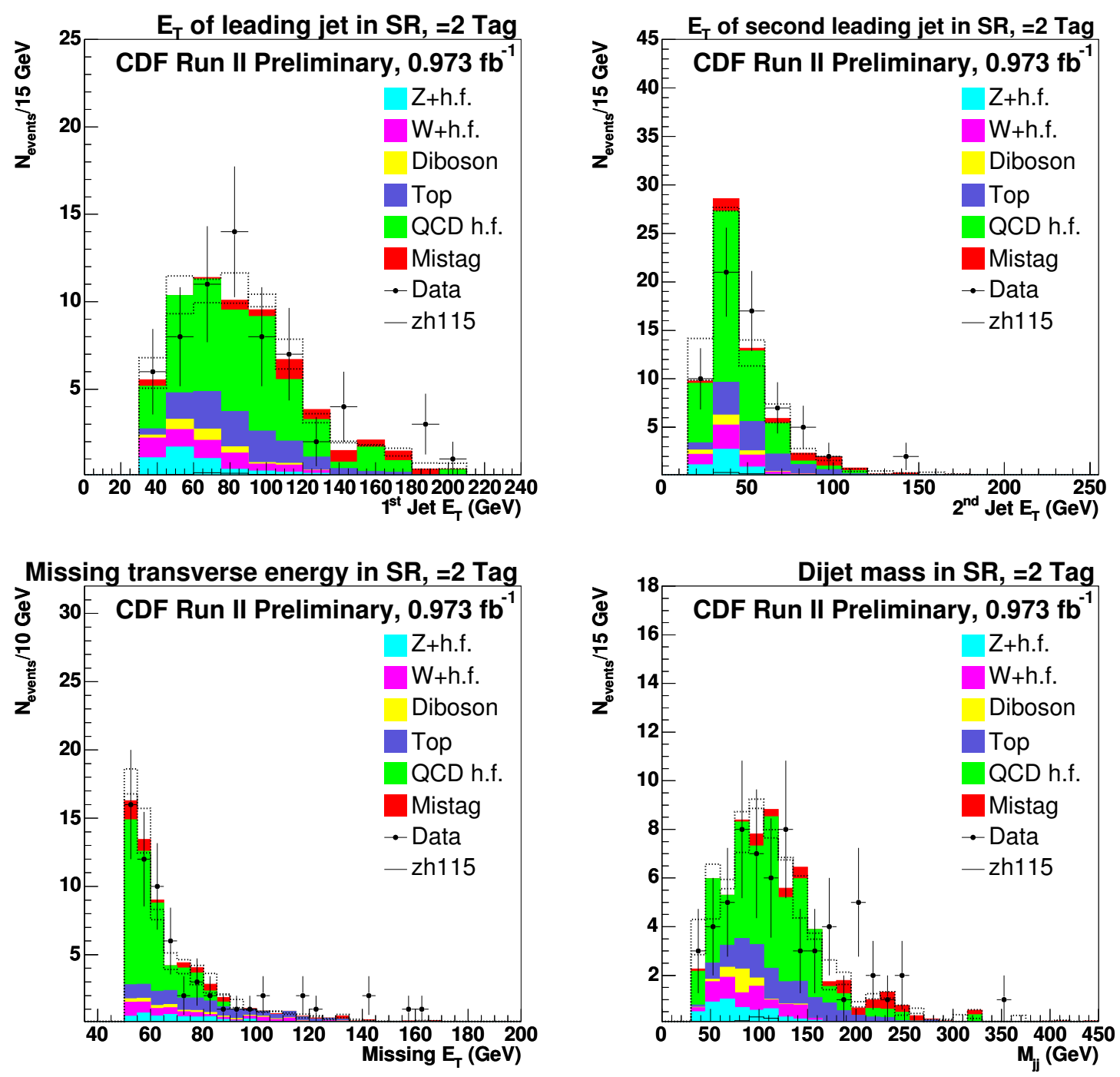

Fig. 6.17. Signal Region, double tag before final selection: distribution of variables $1^{\text {st }}$ jet $E_{T}, 2^{\text {nd }}$ jet $E_{T}, \notin_{T}$, dijet mass 

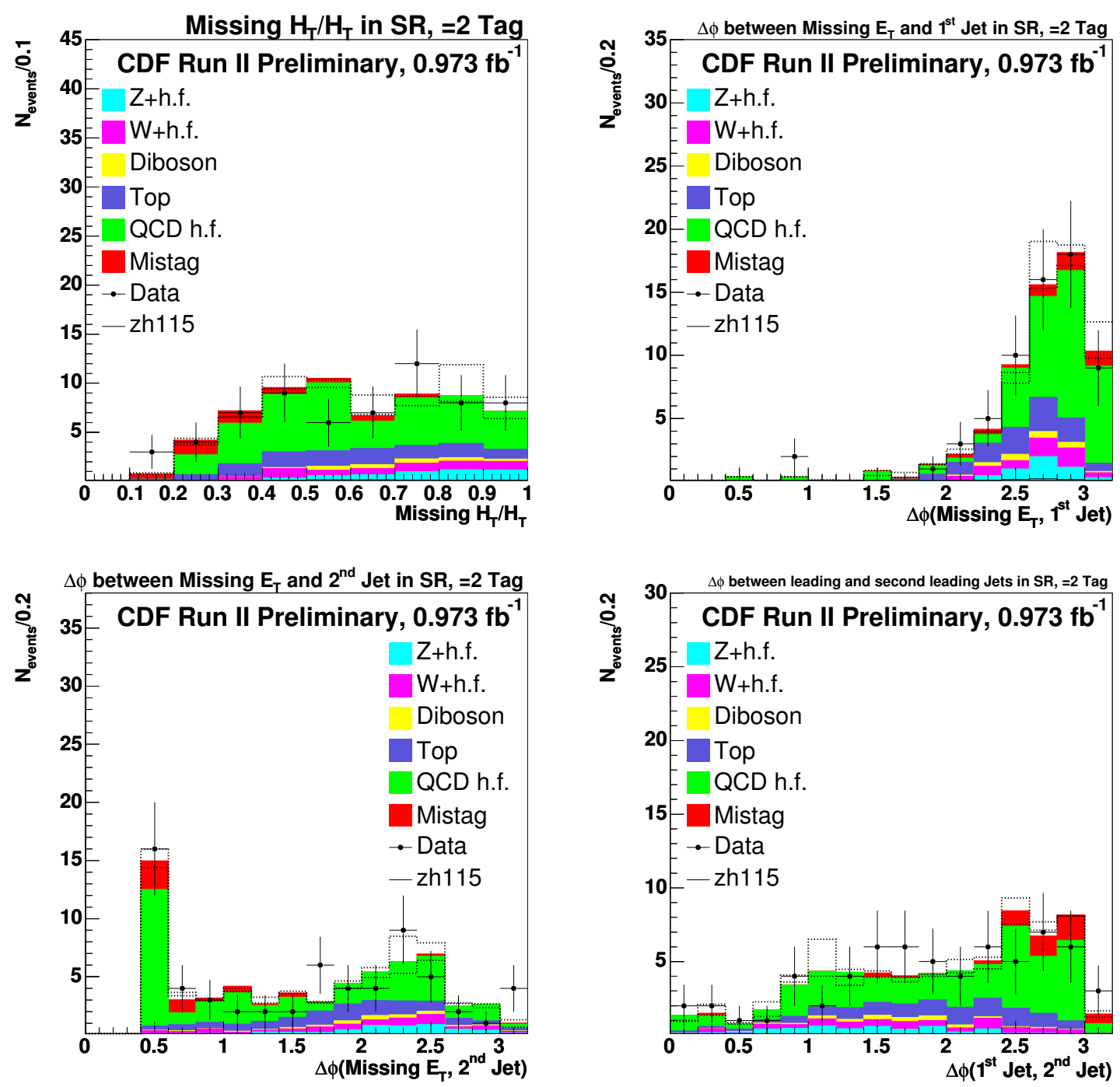

Fig. 6.18. Signal Region, double tag before final selection: distribution of variables $\frac{\not_{T}}{H_{T}}, \varphi\left(1^{s t} j e t, \notin_{T}\right), \varphi\left(2^{\text {nd }}\right.$ jet, $\left.\notin_{T}\right), \Delta \phi\left(1^{\text {st }} j e t, 2^{\text {nd }}\right.$ jet $)$ 
Table 6.6

Acceptance of data and Monte Carlo events in the Signal Region before final optimization

\begin{tabular}{|l|c|c|c|c|c|c|}
\hline & $\begin{array}{c}\text { MET } \\
\text { Clean-up } \\
(\%)\end{array}$ & $\begin{array}{c}\text { Pre- } \\
\text { selection } \\
(\%)\end{array}$ & $\begin{array}{c}\text { Electron } \\
\text { Veto } \\
(\%)\end{array}$ & $\begin{array}{c}\text { IsoTrack } \\
\text { Veto } \\
(\%)\end{array}$ & $\begin{array}{c}\text { SecVtx } \\
=1 \text { Tag } \\
(\%)\end{array}$ & $\begin{array}{c}\text { SecVtx } \\
=2 \text { Tags } \\
(\%)\end{array}$ \\
\hline \hline Data & 60.2 & 2.87 & 2.80 & 2.74 & 0.29 & 0.02 \\
\hline \hline QCD mutli-jet & 71.3 & 0.81 & 0.80 & 0.80 & 0.19 & 0.014 \\
\hline$t \bar{t}$ & 88.5 & 4.6 & 2.89 & 1.63 & 0.67 & 0.15 \\
single top (s-ch.) & 86.4 & 5.88 & 4.56 & 3.06 & 1.37 & 0.43 \\
single top (t-ch.) & 83.1 & 4.08 & 3.14 & 2.10 & 0.87 & 0.008 \\
\hline$W^{ \pm}$to $e^{ \pm} \nu$ & 46.2 & 0.26 & 0.11 & 0.11 & 0.01 & 0.001 \\
$W^{ \pm}$to $\mu^{ \pm} \nu$ & 52.24 & 2.51 & 2.47 & 1.24 & 0.13 & 0.01 \\
$W^{ \pm}$to $\tau^{ \pm} \nu$ & 46.3 & 1.1 & 1.03 & 0.99 & 0.03 & 0.001 \\
\hline Z to $e^{-} e^{+}$ & 49.8 & 0.03 & 0.01 & 0.01 & $10-3$ & 0 \\
Z to $\mu^{-} \mu^{+}$ & 13.7 & 0.25 & 0.24 & 0.06 & 0.01 & 0.001 \\
Z to $\tau^{-} \tau^{+}$ & 49.1 & 1.32 & 1.15 & 0.87 & 0.03 & 0.0002 \\
Z to $\nu \bar{\nu}$ & 52.8 & 3.52 & 3.50 & 3.50 & 0.6 & 0.067 \\
\hline WW to all & 76.6 & 3.85 & 2.90 & 2.02 & 0.09 & 0.001 \\
WZ to all & 74.2 & 3.76 & 3.2 & 2.54 & 0.18 & 0.031 \\
ZZ to all & 45.0 & 1.72 & 1.66 & 1.44 & 0.15 & 0.039 \\
\hline \hline ZH(115) & 80.6 & 20.7 & 20.54 & 20.53 & 9.3 & 3.19 \\
WH(115) & 83.3 & 14.78 & 11.46 & 7.21 & 3.22 & 1.05 \\
\hline
\end{tabular}

- $\notin_{T}>70 \mathrm{GeV}$.

The normalization of both signal and background Monte Carlo is performed by reweighting the events with the production cross-section, scaling them with the trigger efficiency, and applying the b-tagging scale factor that corrects the b-tagging efficiency 
in the simulation. Events must be normalized after the preselection cuts, as the trigger efficiency is not defined earlier. The normalization actually happens after splitting the events into single and double tag categories for convenience, because the final event weight also depends on the number of tags. The number of events after the final selection is shown in Table 6.8. We expect a total of 1.32 Higgs events (with mass $115 \mathrm{GeV}$ ) in the single and 0.47 in the double tag data produced in the $\mathrm{ZH}$ and WH channels together. Figure 6.19 shows the dijet mass distributions in the single and double tags after final selection.

Table 6.7

Number of expected and observed background events in the Signal Region before applying the final cuts.

\begin{tabular}{|l|c|c|}
\hline Process & Single Tag & Double Tag \\
\hline \hline QCD multi-jet & $1046 \pm 197$ & $35.2 \pm 8.6$ \\
TOP & $61.4 \pm 8.9$ & $10.9 \pm 1.8$ \\
Di-boson & $17.3 \pm 3.1$ & $1.97 \pm 0.41$ \\
W + h.f. & $158 \pm 85$ & $5.19 \pm 2.63$ \\
Z + h.f. & $62.2 \pm 27.1$ & $5.13 \pm 2.29$ \\
Mistag & $276 \pm 11$ & $5.35 \pm 2.26$ \\
\hline \hline Expected & $1623 \pm 287$ & $64.5 \pm 13.2$ \\
\hline Observed & 1566 & 64 \\
\hline
\end{tabular}



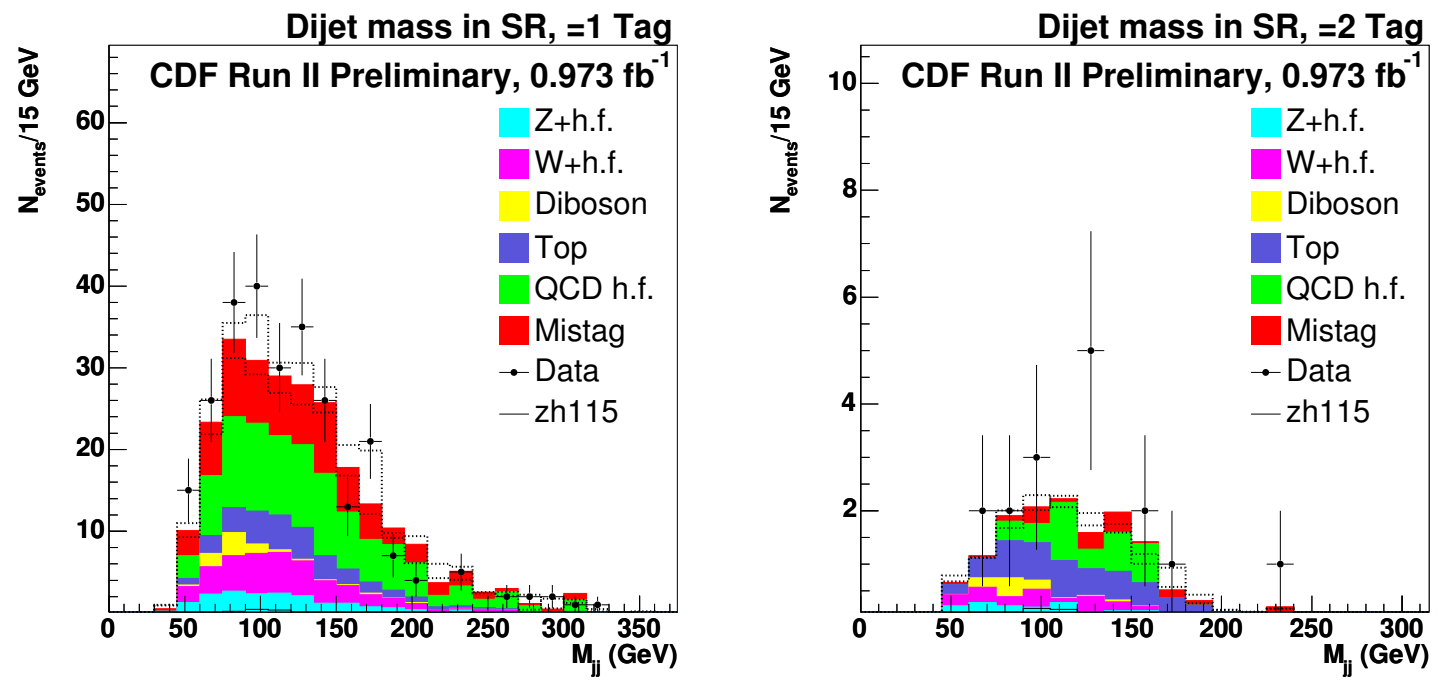

Fig. 6.19. Dijet invariant mass in the Signal Region after final selection, single- and double-tagged events. The dashed line shows the $\pm 1 \sigma$ variation in the Jet Energy Scale uncertainty. 
Table 6.8

Number of expected and observed background and signal events in the Signal Region after applying the final cuts.

\begin{tabular}{|l|c|c|}
\hline Process & Single Tag & Double Tag \\
\hline \hline QCD multi-jet & $93 \pm 23$ & $3.65 \pm 1.27$ \\
TOP & $27.3 \pm 3.8$ & $4.91 \pm 0.80$ \\
Di-boson & $7.0 \pm 1.4$ & $0.79 \pm 0.19$ \\
W + h.f. & $33.4 \pm 16.2$ & $1.65 \pm 0.86$ \\
Z + h.f. & $18.3 \pm 8.1$ & $1.67 \pm 0.77$ \\
Mistag & $71 \pm 10$ & $1.70 \pm 0.48$ \\
\hline ZH (115) & $0.70 \pm 0.078$ & $0.25 \pm 0.033$ \\
WH (115) & $0.53 \pm 0.059$ & $0.19 \pm 0.031$ \\
\hline \hline Expected & $251 \pm 43$ & $14.8 \pm 2.7$ \\
\hline Observed & 268 & 16 \\
\hline
\end{tabular}

\subsection{Systematic Uncertainties}

Systematic uncertainties are uncertainties in the signal and background predictions that do not scale simply with luminosity ${ }^{3}$. They are treated as random errors, although they are not always associated with random effects. The sources of the systematic uncertainties are sometimes not well identified due to the complexity of the problem. In such cases, estimating uncertainty from first principles is not possible, therefore we provide our best estimate based on measurements or simulation.

Uncertainties are classified as either correlated or uncorrelated considering if they affect some or all the signal and background processes simultaneously or independently. These uncertainties are regarded as either totally correlated or not correlated

\footnotetext{
${ }^{3}$ Having more data often leads to smaller uncertainties due to better understanding of the systematics. Dividing data into bins, however, does not necessarily change the uncertainty. This is the main difference between systematic uncertainty and statistical error.
} 
at all. Table 6.10 lists the uncertainties for the single and Table 6.11 for the double tag signal regions after applying all final selection cuts. These uncertainties are as follows:

- Luminosity Uncertainty. The procedure for determining the error on the luminosity measurement for Run 2 is described in [58]. This uncertainty amounts to $6 \%$ and applies to those simulations that are normalized to luminosity. This uncertainty therefore is not applied directly on the QCD.

- PDF uncertainty. The PDF uncertainty has been determined only for the signal using the method that is described in [59]. In earlier analyses (see [54]), it has been determined that a $2 \%$ uncertainty on the acceptance due to the choice of the PDF is sufficient.

The MC reweighting method using CTEQ5L [60] provides $+0.85 \%$ and $-1.00 \%$ for $\mathrm{ZH}$ at $115 \mathrm{GeV}$ and $+1.77 \%$ and $-1.01 \%$ for $\mathrm{WH}$ at $115 \mathrm{GeV}$ is. We assign and overall $2 \%$ to all Monte Carlo samples.

- Jet Energy Scale Uncertainty. A systematic error in the calorimeter energy scale can affect the total transverse energy of jets. The Jet Energy Scale uncertainty was determined by varying the jet energy correction factor by plus and minus one sigma while correcting the transverse energy of the jets. This variation then propagates to the $\mathscr{E}_{T}$ reconstruction, the calculation of the azimuthal direction of $\notin_{T}$ and thus to the expected number of events after applying the selection cuts. The influence of this variation is different for each background component; therefore, it needs to be determined separately by running the entire analysis code twice on all the simulated backgrounds.

The JES uncertainty can also modify the shape of the distributions. It is taken into account in the limit calculation for each background channels separately and is also is propagated into the errors displayed in the data-MC comparison plots. 
In some cases, the JES can be very asymmetric. This happens, for example, after the final selection in the single tag signal region. When the two errors are not much different, we always take the larger one. In the limit calculation, the $\pm 1 \sigma$ shapes are provided for each backgrounds and signal, thus the asymmetric nature of the uncertainty is also taken into account.

- QCD multi-jet background normalization. The systematic error on the normalization is computed as explained above giving $4 \%$ in the single and $4.8 \%$ in the double tag region.

- B-tagging scale factor. The efficiency of tagging a taggable jet in the simulation is about $5 \%$ larger than in real events. This difference has to be taken into account when calculating the predicted number of events in the simulation after requiring a tag. For the tight tagger the correct scale factor is $0.95 \pm 0.01$ (stat) \pm 0.04 (syst) described in Section 3.5, therefore we use a $4.3 \%$ error for all the simulated background components in the single and $8.6 \%$ in the double tag region.

- SecVtx Tag Rate Matrix (see Section 5.2.4) systematics. The total systematic error in estimating the negative tight b-tags by the tag rate matrix is $4.8 \%$. This uncertainty is lumped into the total Mistag rate error.

- Mistag rate (asymmetry factor). The asymmetry factor, about 1.44, is applied to the number of negative tags to obtain the number of positive mistags. The exact $\alpha \beta$ correction depends on the $E_{T}$ of the jet. It is implemented in the Tag Rate matrix when using the MistagRate prediction. The absolute error assigned to the rate of each jet requested from the Matrix contains the error in the previous bullet, the uncertainty in measuring the $\alpha \beta$ correction, and the statistical error in the matrix element. The statistical error in the sample on which the matrix was applied was propagated while running on the matrix. 
- W/Z+h.f. and Diboson Monte Carlo NLO corrections. We use a $40 \%$ uncertainty on the $\mathrm{W}$ and $\mathrm{Z}$ background prediction and $11.5 \%$ on the diboson as described in Section 5.2.3.

- Trigger Efficiency. The trigger efficiency study is described in Section 4.2. The uncertainty on the trigger efficiency is $3 \%$ in the signal region where $E_{T}>70 \mathrm{GeV}$.

- Lepton Veto. The uncertainty on the lepton veto is $2 \%$ (Section 6.5).

- ISR/FSR. The uncertainty associated with the initial and final state radiations is determined from the signal simulation generated with Higgs mass of $115 \mathrm{GeV}$ and more or less ISR or FSR according to the standard CDF recommendations. The uncertainty on the acceptance was evaluated for the $\mathrm{ZH}$ and $\mathrm{WH}$ signals separately by running the full analysis on these samples. Table 6.9 lists all possible combinations. Note that the signal acceptance can change in both negative and positive directions due to varying the amount of radiation in the simulation. In the limit calculation we use the average uncertainties in the single and double tags separately. 
Table 6.9

ISR/FSR uncertainties

\begin{tabular}{|c|c|c|c|c|}
\hline Process & \multicolumn{2}{|c|}{ Single Tag } & \multicolumn{2}{c|}{ Double Tag } \\
\hline \hline ZH (115 GeV) & more & less & more & less \\
\hline ISR & $+1.8 \%$ & $-2.4 \%$ & $+4.9 \%$ & $-2.6 \%$ \\
FSR & $+0.1 \%$ & $-0.6 \%$ & $-0.6 \%$ & $+1.5 \%$ \\
\hline \hline WH (115 GeV) & more & less & more & less \\
\hline ISR & $+3.5 \%$ & $-0.3 \%$ & $+1.8 \%$ & $+2.4 \%$ \\
FSR & $+2.3 \%$ & $-1.3 \%$ & $+4.2 \%$ & $+0.4 \%$ \\
\hline \hline Average & more & less & more & less \\
\hline ISR & $+2.5 \%$ & $-1.5 \%$ & $+3.5 \%$ & $-0.5 \%$ \\
FSR & $+1.1 \%$ & $-1.0 \%$ & $+1.5 \%$ & $+1.0 \%$ \\
\hline
\end{tabular}


Table 6.10

Summary of systematic uncertainties in the Single Tag Signal Region after final cuts. (See also Table 6.9)

\begin{tabular}{|l|c|c|c|c|c|c|c|c|}
\hline & $\begin{array}{c}\text { ZH } \\
(\%)\end{array}$ & $\begin{array}{c}\text { WH } \\
(\%)\end{array}$ & $\begin{array}{c}\text { QCD } \\
(\%)\end{array}$ & $\begin{array}{c}\text { Top } \\
(\%)\end{array}$ & $\begin{array}{c}\text { Diboson } \\
(\%)\end{array}$ & $\begin{array}{c}\text { W+h.f. } \\
(\%)\end{array}$ & $\begin{array}{c}\text { Z+h.f. } \\
(\%)\end{array}$ & $\begin{array}{c}\text { Mistag } \\
(\%)\end{array}$ \\
\hline \hline Correlated Uncertainty \\
\hline \hline Luminosity & 6.0 & 6.0 & & 6.0 & 6.0 & 6.0 & 6.0 & \\
Tagging SF & 4.3 & 4.3 & 4.3 & 4.3 & 4.3 & 4.3 & 4.3 & \\
Jet Energy Sc. & 8.0 & 8.0 & 23.0 & 0.1 & 14.0 & 26.0 & 16.0 & \\
Trigger Eff. & 3.0 & 3.0 & 3.0 & 3.0 & 3.0 & 3.0 & 3.0 & \\
Lepton Veto & 2.0 & 2.0 & 2.0 & 2.0 & 2.0 & 2.0 & 2.0 & 2.0 \\
PDF accept. & 2.0 & 2.0 & 2.0 & 2.0 & 2.0 & 2.0 & 2.0 & \\
\hline \hline Uncorrelated Uncertainty & & & 11.0 & 11.5 & 40.0 & 40.0 & \\
\hline \hline Cross-section & & & 3.1 & & & & & \\
QCD Norm.
\end{tabular}


Table 6.11

Summary of systematic uncertainties in the Double Tag Signal Region after final cuts. (See also Table 6.9)

\begin{tabular}{|c|c|c|c|c|c|c|c|c|}
\hline & $\begin{array}{l}\mathrm{ZH} \\
(\%)\end{array}$ & $\begin{array}{l}\text { WH } \\
(\%)\end{array}$ & $\begin{array}{l}\text { QCD } \\
(\%)\end{array}$ & $\begin{array}{l}\text { Top } \\
(\%)\end{array}$ & $\begin{array}{c}\text { Diboson } \\
(\%)\end{array}$ & $\begin{array}{c}W+\text { h.f. } \\
(\%)\end{array}$ & $\begin{array}{c}\text { Z+h.f. } \\
(\%)\end{array}$ & $\begin{array}{c}\text { Mistag } \\
(\%)\end{array}$ \\
\hline \multicolumn{9}{|c|}{ Correlated Uncertainty } \\
\hline Luminosity & 6.0 & 6.0 & & 6.0 & 6.0 & 6.0 & 6.0 & \\
\hline Tagging SF & 8.7 & 8.7 & 8.7 & 8.7 & 8.7 & 8.7 & 8.7 & \\
\hline Jet Energy Sc. & 7.0 & 7.0 & 10.0 & 1.0 & 15.0 & 25.0 & 17.0 & \\
\hline Trigger Eff. & 3.0 & 3.0 & 3.0 & 3.0 & 3.0 & 3.0 & 3.0 & \\
\hline Lepton Veto & 2.0 & 2.0 & 2.0 & 2.0 & 2.0 & 2.0 & 2.0 & 2.0 \\
\hline PDF accept. & 2.0 & 2.0 & 2.0 & 2.0 & 2.0 & 2.0 & 2.0 & \\
\hline \multicolumn{9}{|c|}{ Uncorrelated Uncertainty } \\
\hline Cross-section & & & & 11.0 & 11.5 & 40.0 & 40.0 & \\
\hline QCD Norm. & & & 4.8 & & & & & \\
\hline Mistag Est. & & & & & & & & 90.0 \\
\hline MC statistics & 2.0 & 2.0 & 32.0 & 3.0 & 9.0 & 20.0 & 11.0 & \\
\hline
\end{tabular}




\section{INTERPRETATION OF THE RESULTS}

The number of events predicted and observed in the signal region after final selection are shown in Table 6.8. No significant excess is found in the data with respect to the predicted background; therefore, there is no indication for the presence of undiscovered physics. The outcome of the experiment is consistent with the Standard Model with or without a SM Higgs boson.

We quantify the last statement by asking statistically how many events the Higgs could yield such that the observed data is consistent with the sum of the assumed signal and the background prediction. This number can be anything between zero and a certain maximum. The maximum is determined by the required level of consistency with the data. We use a Bayesian approach to calculate the upper limit when the Bayesian confidence level is 95\%. This means that with the given background prediction $b$, observed event number $n$, and expected signal efficiency $\epsilon$, the probability that a signal process exists with any number of events $s$ between 0 and $s_{u}$ is $95 \%$. In other words, there is only a $5 \%$ chance that a new process is present in the data with $\epsilon s_{u}$ or more events on average.

\subsection{Limit Calculation}

Rather than performing a single counting experiment, we use the dijet mass histogram in Figure 6.19 to compute the upper limit. Each bin of the histogram is considered a separate channel in the calculation with its own background and signal efficiency. It is often worthwhile to divide the data into multiple channels especially if some channels have better signal separation than the whole sample. After combining the individual channel limits, no signal is discarded, and the combined limit is at least as good or better than the result of a counting experiment. A similar division 
is made when we split the data into single and double tag events. The double tag rate for $\mathrm{ZH} / \mathrm{WH}$ is larger than for most of the background processes; therefore, the double tag sample provides a better signal to background ratio for about $40 \%$ of the signal events. Thus the number of channels $N$ is the sum of the number of bins in the two dijet mass histograms.

The limit calculation is done with a software package described in [61]. It contains, among other features, a Higgs-search specific implementation of a multi-channel Bayesian limit calculator documented in [62]. In the Bayesian approach, the signal efficiencies $\bar{\epsilon}=\left(\epsilon_{1}, \ldots, \epsilon_{N}\right)$ and background predictions $\bar{b}=\left(b_{1}, \ldots, b_{N}\right)$ are treated as nuisance parameters with associated systematic uncertainties. The $95 \%$ upper limit is provided by solving the following integral for $s_{u}$ :

$$
\int_{0}^{s_{u}} p(s) d s=0.95
$$

where $s$ is the number of signal events and $p(s)$ is its marginalized posterior. The posterior is constructed based on Bayes' Theorem assuming that $n_{k}$ is Poisson distributed with mean $s \epsilon_{k}+b_{k}$. After "marginalizing" the nuisance parameters, the posterior of $s$ is proportional to

$$
p(s) \sim \pi(s) \int_{(2 N)} \pi(\bar{\epsilon}, \bar{b})\left[\prod_{k=1}^{N} \frac{e^{-\left(s \epsilon_{k}+b_{k}\right)}\left(s \epsilon_{k}+b_{k}\right)^{n_{k}}}{n_{k} !}\right] d \bar{\epsilon} d \bar{b}
$$

where $\pi(s)$ is the prior of $s$ chosen to be constant, and $\pi(\bar{\epsilon}, \bar{b})$ is a joint prior of the nuisance parameters which is obtained from subsidiary measurements or simulations. They are generally Poisson distributions, although they may be treated as Gaussian in certain cases. The formula is evaluated by Monte Carlo integration. In this method, a set of $\bar{b}$ and $\bar{\epsilon}$ parameters are generated $M=1000$ times randomly according to the prior p.d.f we choose for the nuisance parameters. The marginalized posterior becomes the product in the brackets of Equation (7.2) averaged over the $M$ trials and multiplied by $\pi(s)$. The correlations between the nuisance parameters and their uncertainties are taken into account when generating the random vectors. The integration over $s$ is evaluated numerically. For a detailed explanation of the method we refer to $[62]$. 
The software documented in [61] contains a framework for setting up the background predictions, the expected signal acceptances, and the correlated and uncorrelated uncertainties of the nuisance parameters. Before giving a brief description of the input variables required by the program, we need to introduce some notations.

The yield of a process with cross-section $\sigma$ is given by the following formula:

$$
n_{\text {data }}=\hat{a} \cdot \epsilon_{\text {trigger }} \cdot \sigma \cdot \mathcal{L}_{\text {data }}
$$

where $\hat{a}$ is the acceptance of all the offline selection cuts, $\epsilon_{\text {trigger }}$ is the efficiency of the online selection, and $N_{\text {data }}=\sigma \cdot \mathcal{L}_{\text {data }}$ is the number of events in $\mathcal{L}_{\text {data }}$ amount of collision data. This is represented in the simulation by $N_{M C}=\sigma \cdot \mathcal{L}_{M C} \neq N_{\text {data }}$ events as

$$
n_{\text {data }} \sim(f \cdot \hat{a}) \cdot \epsilon_{\text {trigger }} \cdot N_{M C}
$$

where $f$ is an overall scale factor that corrects the different selection efficiencies between data and simulation. Using simulation has the advantage that the statistical error in the prediction can be reduced by requiring $\mathcal{L}_{M C}>>\mathcal{L}_{\text {data }}$. This is satisfied for the signal and for most of the backgrounds (see Table 5.2). Thus the Monte Carlo prediction for $n_{\text {data }}$ is given by

$$
n_{\text {pred }}=(f \cdot \hat{a}) \cdot \epsilon_{\text {trigger }} \cdot w \cdot N_{M C}
$$

where $w=\mathcal{L}_{\text {data }} / \mathcal{L}_{M C}$. In the signal region of this analysis, $f, \epsilon_{\text {trigger }}$, and $w$ are all assumed to be constants within their uncertainties.

The Monte Carlo simulates the $\hat{a} \cdot N_{M C}$ part of Equation (7.5). It yields an integral number of Monte Carlo events passing all selections which follow a Poisson distribution. The systematic uncertainty represented by the statistical error of $\hat{a} \cdot N_{M C}$ also scales with $f \cdot \epsilon_{\text {trigger }} \cdot w$.

Thus the following information is needed to compute the limits:

- $h_{k}=\hat{a_{k}} \cdot N_{M C}^{k}$ histograms predicting the background in the dijet mass distributions, 
- overall scale factor $f \cdot \epsilon_{\text {trigger }} \cdot w$ for every histogram; thus the total background prediction is $b_{k}=f \cdot \epsilon_{\text {trigger }} \cdot w \cdot h_{k}$,

- signal prediction. This is also given by histograms with bins $s_{k}=\left(f \cdot \hat{a}_{k}\right)$. $\epsilon_{\text {trigger }} \cdot w \cdot N_{M C}^{k}$. The nuisance parameters are calculated by

$$
\epsilon_{k}=\frac{s_{k}}{\sum_{k=1}^{N} s_{k}}
$$

- systematic uncertainties for each scale factor:

1. lepton veto, b-tagging efficiency, propagated uncertainties of analysis cuts due to JES, and PDF uncertainties are assigned for $f$ (the ratio of the offline selection efficiencies between data and simulation),

2. trigger efficiency uncertainty for $\epsilon_{\text {trigger }}$,

3. luminosity measurement, cross-section or QCD normalization uncertainties for the sample normalization $w$,

- the mistag prediction. In this case, the total propagated uncertainty is calculated together with the central prediction. The prior p.d.f is assumed to be a Gaussian.

The uncertainties that are correlated with the dijet mass can change the shape of the dijet mass prediction, therefore an uncertainty must be specified for each channel. In this analysis, we consider the Jet Energy Scale the only example of such an uncertainty assigned to the simulated processes and the mistag uncertainty for the mistag background. These are specified by histograms with the same binning as the central prediction.

The $95 \%$ C.L. upper limit is set on the number of events $s_{u}$ that is allowed to be produced by an undiscovered process which has kinematic distributions identical to the Higgs production. The ratio

$$
R=\frac{s_{u}}{\sum_{k=1}^{N} s_{k}}=\frac{\sigma_{u}}{\sigma_{\text {SMHiggs }}}
$$


translates the excluded cross-section of $s$ to the excluded Standard Model Higgs crosssection.

The expected values for $R$ are calculated assuming that the Higgs boson does not exist, therefore the mean of $n_{k}$ is $b_{k}$ in every bin. The quoted numbers, $R_{\text {expected }}$, are the mean values of $R$ computed in multiple trials. The errors on $R_{\text {expected }}$ are the intervals that contain $68 \%$ of these trials. The values $R_{\text {observed }}$ are determined by the number of events observed in the data.

\subsection{Results and Future Prospects}

After normalizing the signal prediction with the cross-sections from Table 5.1, the number of signal events is expected to be more than an order of magnitude smaller than the background. It is also smaller than the systematic error on the background prediction. In order to make a more stringent statement about the existence of the SM Higgs boson more data is needed to reduce both the statistical fluctuations and the sizes of the systematic uncertainties.

We can use the ratio $R_{\text {expected }}$ between the expected excluded cross-section and the SM predicted cross-section for the $\mathrm{ZH} / \mathrm{WH}$ processes to estimate how much data is needed to test the Standard Model. This happens when $R_{\text {expected }} \approx 1$. In the most pessimistic scenario, if the analysis tools and our level of understanding the detector were not going to improve, then $R_{\text {expected }}$ would be inverse proportional to the improvement in luminosity. Therefore, we would need to acquire about two orders of magnitude more collision data if we relied only on Higgs events produced with Z/W bosons and detected in the $\mathbb{E}_{T}$ and b-jets events. Tables 7.1 and 7.2 show $R$ in the $\mathrm{ZH}$ and WH production channels for single tag, double tag, and the whole sample after combining the individual limits. Table 7.3 shows the final result where the $\mathrm{ZH}$ and $\mathrm{WH}$ channels are combined to $\mathrm{VH}$ also in the three cases of single tags, double tags, and combined sample. The limit ratios are plotted in Figure 7.1. 

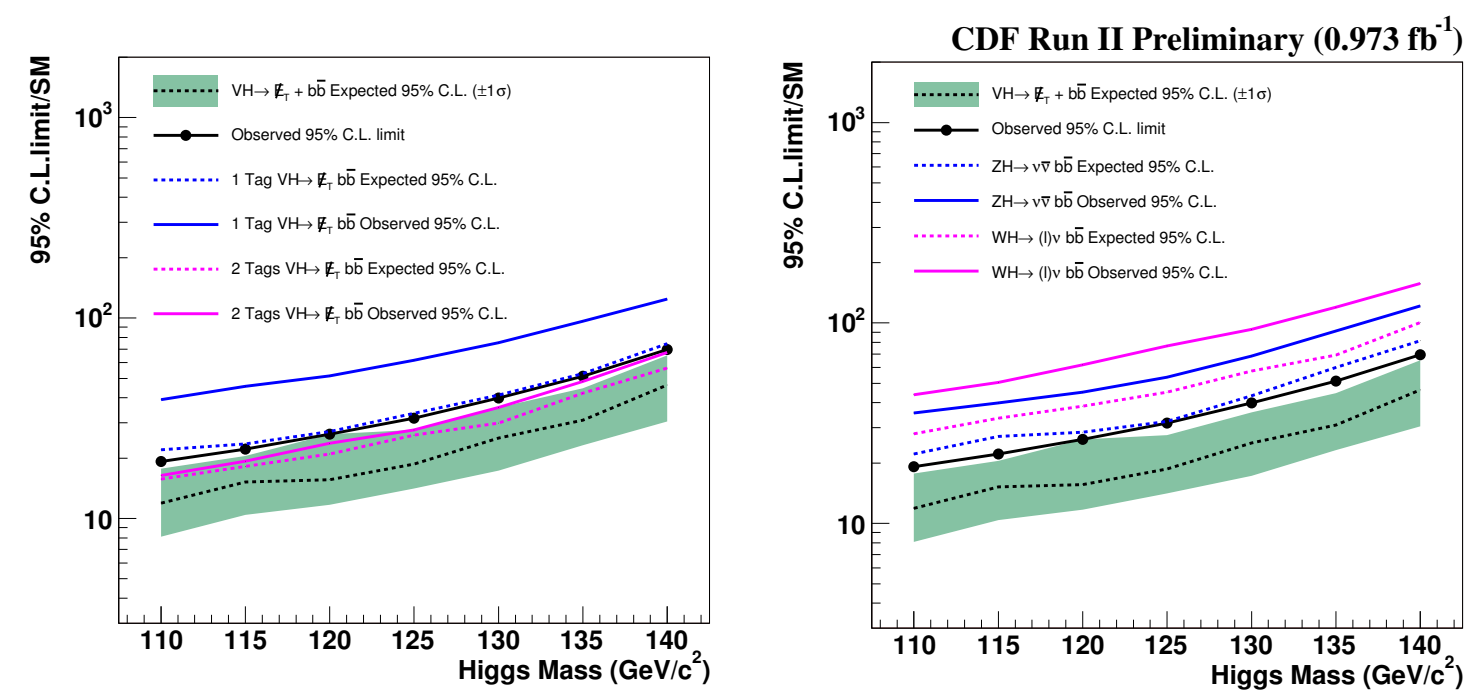

Fig. 7.1. Left: exclusion limits in the exclusive single, double, and combined tags with respect to the SM prediction. Right: exclusion limits in $\mathrm{WH}, \mathrm{ZH}$, and the combination $\mathrm{VH}$ with respect to the $\mathrm{SM}$ prediction.

The computed ratios indicate the sensitivity represented by the analyzed channel as well as the relative sensitivity of this Higgs search with respect to other searches. The combined Higgs searches at CDF have been described in [63] (Figure 7.2), the total Tevatron sensitivity is presented in [64]. The total Tevatron sensitivity for a light SM Higgs and the combined channels are shown in Figure 7.3. We observe a reduction in $R_{\text {expected }}$ after the combination that is equivalent to multiplying the data by about a factor of 4 . Improvements in the analysis technique and combinations of more search channels will further improve the Tevatron sensitivity.

Table 7.4 shows the absolute limits in the $\mathrm{ZH}, \mathrm{WH}$, and combined $\mathrm{VH}$ production channels (last columns in Table 7.1, Table 7.2, and Table 7.3). They are computed from:

$$
\sigma_{u}=R \cdot \sigma_{\text {SMHiggs }}(Z H / W H / V H) \cdot B r(H \rightarrow b \bar{b})
$$


Table 7.1

The expected and observed cross-section limits obtained in ZH processes divided by the SM cross-section for $H \rightarrow b \bar{b}$ and $Z \rightarrow \nu \bar{\nu}$ for single tag, double tag events and their combination.

\begin{tabular}{|c|c|c|c|c|c|c|}
\hline \multirow{2}{*}{$\begin{array}{l}\text { Higgs mass } \\
\text { (GeV) }\end{array}$} & \multicolumn{2}{|c|}{$\begin{array}{c}\sigma(Z H \rightarrow \nu \bar{\nu} b \bar{b}) / S M \\
\text { Exclusive } 1 \text { Tag }\end{array}$} & \multicolumn{2}{|c|}{$\begin{array}{c}\sigma(Z H \rightarrow \nu \bar{\nu} b \bar{b}) / S M \\
\text { Double Tag }\end{array}$} & \multicolumn{2}{|c|}{$\begin{array}{c}\sigma(Z H \rightarrow \nu \bar{\nu} b \bar{b}) / S M \\
\text { Combined }\end{array}$} \\
\hline & Expected & Observed & Expected & Observed & Expected & Observed \\
\hline 110 & $36.8_{-9.1}^{+16.6}$ & 69.5 & $26.8_{-6.5}^{+10.6}$ & 29.6 & $22.2_{-6.1}^{+11.0}$ & 35.5 \\
\hline 115 & $41.2_{-11.6}^{+18.9}$ & 77.2 & $32.2_{-9.5}^{+15.6}$ & 33.7 & $27.3_{-9.6}^{+12.7}$ & 39.9 \\
\hline 120 & $50.1_{-15.9}^{+22.1}$ & 91.8 & $38.9_{-11.9}^{+15.8}$ & 39.5 & $28.5_{-7.5}^{+11.6}$ & 45.1 \\
\hline 125 & $60.7_{-18.3}^{+23.2}$ & 101.5 & $42.5_{-11.4}^{+17.4}$ & 48.3 & $32.2_{-7.7}^{+17.5}$ & 53.6 \\
\hline 130 & $72.6_{-19.8}^{+33.3}$ & 132.2 & $55.3_{-15.8}^{+25.3}$ & 64.1 & $43.4_{-13.8}^{+19.1}$ & 68.3 \\
\hline 135 & $88.4_{-27.9}^{+39.5}$ & 162.4 & $72.5_{-18.5}^{+35.8}$ & 87.8 & $59.9_{-16.4}^{+24.4}$ & 91.3 \\
\hline 140 & $129.2_{-35.7}^{+63.7}$ & 222.7 & $96.4_{-24.4}^{+37.0}$ & 116.5 & $81.5_{-26.6}^{+31.8}$ & 121.6 \\
\hline
\end{tabular}

The advantage of quoting the absolute limits is that they are independent of the current theoretical predictions for the production cross-sections and the branching fractions of the Higgs. 
Table 7.2

The expected and observed cross-section limits obtained in WH processes when $H \rightarrow b \bar{b}$ and $\mathrm{W}$ decays leptonically where the lepton is undetected. The limits are divided by the SM cross-section and are presented for single tag, double tag events and their combination.

\begin{tabular}{|c|c|c|c|c|c|c|}
\hline \multirow{2}{*}{$\begin{array}{l}\text { Higgs mass } \\
\qquad(\mathrm{GeV})\end{array}$} & \multicolumn{2}{|c|}{$\begin{array}{c}\sigma(W H \rightarrow l \nu b \bar{b}) / S M \\
\text { Exclusive } 1 \mathrm{Tag}\end{array}$} & \multicolumn{2}{|c|}{$\begin{array}{c}\sigma(W H \rightarrow l \nu b \bar{b}) / S M \\
\text { Double Tag }\end{array}$} & \multicolumn{2}{|c|}{$\begin{array}{c}\sigma(W H \rightarrow l \nu b \bar{b}) / S M \\
\text { Combined }\end{array}$} \\
\hline & Expected & Observed & Expected & Observed & Expected & Observed \\
\hline 110 & $50.8_{-15.5}^{+21.5}$ & 96.0 & $33.6_{-9.3}^{+16.1}$ & 36.8 & $28.0_{-8.0}^{+13.0}$ & 43.8 \\
\hline 115 & $54.3_{-16.3}^{+23.3}$ & 107.3 & $42.1_{-13.4}^{+17.6}$ & 42.8 & $33.5_{-10.8}^{+15.9}$ & 50.5 \\
\hline 120 & $62.1_{-15.4}^{+27.3}$ & 125.1 & $49.3_{-13.5}^{+23.8}$ & 55.6 & $38.5_{-12.9}^{+17.6}$ & 61.8 \\
\hline 125 & $78.3_{-26.1}^{+28.5}$ & 144.6 & $59.2_{-16.2}^{+22.8}$ & 68.5 & $45.1_{-13.4}^{+18.1}$ & 76.0 \\
\hline 130 & $90.0_{-27.4}^{+35.8}$ & 175.7 & $73.9_{-21.6}^{+33.6}$ & 84.9 & $57.5_{-17.2}^{+23.3}$ & 93.1 \\
\hline 135 & $128.8_{-46.6}^{+60.6}$ & 226.3 & $94.6_{-23.7}^{+47.1}$ & 110.2 & $69.1_{-18.7}^{+35.1}$ & 119.9 \\
\hline 140 & $174.2_{-57.7}^{+67.8}$ & 300.2 & $144.6_{-45.3}^{+57.0}$ & 159.5 & $100.6_{-29.4}^{+43.2}$ & 157.4 \\
\hline
\end{tabular}


Table 7.3

The expected and observed cross-section limits of the $\mathrm{ZH}$ and $\mathrm{WH}$ processes combined when $H \rightarrow b \bar{b}, \mathrm{Z}$ decays to neutrinos, and $\mathrm{W}$ decays leptonically divided by the SM cross-section.

\begin{tabular}{|c|c|c|c|c|c|c|}
\hline Higgs & \multicolumn{2}{|c|}{$\sigma\left(V H \rightarrow \phi_{T}, b \bar{b}\right) / S M$} & \multicolumn{2}{|c|}{$\sigma\left(V H \rightarrow \not_{T}, b \bar{b}\right) / S M$} & \multicolumn{2}{|c|}{$\sigma\left(V H \rightarrow \not_{T}, b \bar{b}\right) / S M$} \\
mass & \multicolumn{2}{|c|}{ Exclusive 1 Tag } & \multicolumn{2}{|c|}{$\begin{array}{c}\text { Double Tag } \\
\text { Combined }\end{array}$} \\
\hline \hline $\mathrm{GeV})$ & Expected & Observed & Expected & Observed & Expected & Observed \\
\hline 110 & $22.0_{-6.6}^{+9.3}$ & 39.1 & $15.7_{-4.6}^{+6.7}$ & 16.4 & $11.9_{-3.8}^{+5.8}$ & 19.2 \\
115 & $23.5_{-7.3}^{+12.0}$ & 45.6 & $18.2_{-4.5}^{+9.1}$ & 19.3 & $15.2_{-4.8}^{+5.3}$ & 22.2 \\
120 & $27.1_{-8.2}^{+12.9}$ & 51.4 & $21.0_{-5.4}^{+11.3}$ & 23.7 & $15.6_{-3.9}^{+10.7}$ & 26.3 \\
125 & $33.4_{-11.2}^{+12.6}$ & 61.6 & $26.0_{-7.9}^{+10.1}$ & 27.6 & $18.7_{-4.6}^{+8.9}$ & 31.6 \\
130 & $41.3_{-12.1}^{+20.3}$ & 75.2 & $29.9_{-8.5}^{+10.7}$ & 35.8 & $25.2_{-7.9}^{+10.6}$ & 39.9 \\
135 & $52.8_{-14.7}^{+23.9}$ & 96.5 & $42.2_{-11.1}^{+19.6}$ & 48.3 & $30.9_{-7.7}^{+13.7}$ & 51.2 \\
140 & $74.4_{-24.3}^{+34.6}$ & 124.1 & $56.3_{-14.5}^{+29.7}$ & 67.3 & $46.3_{-15.8}^{+18.9}$ & 69.5 \\
\hline
\end{tabular}

Table 7.4

The expected and observed cross-section limits of the $\mathrm{ZH}, \mathrm{WH}$, and combined processes considering only $H \rightarrow b \bar{b}$ decays. (Single and double tags are also combined.)

\begin{tabular}{|c|c|c|c|c|c|c|}
\hline Higgs & \multicolumn{2}{|c|}{$\sigma(Z H) * B r(H \rightarrow b \bar{b})$} & \multicolumn{2}{c|}{$\sigma(W H) * B r(H \rightarrow b \bar{b})$} & \multicolumn{2}{c|}{$\sigma(V H) * B r(H \rightarrow b \bar{b})$} \\
mass & Expected & Observed & Expected & Observed & Expected & Observed \\
$(\mathrm{GeV})$ & $(\mathrm{pb})$ & $(\mathrm{pb})$ & $(\mathrm{pb})$ & $(\mathrm{pb})$ & $(\mathrm{pb})$ & $(\mathrm{pb})$ \\
\hline \hline 110 & $2.1_{-0.6}^{+1.1}$ & 3.4 & $4.7_{-1.3}^{+2.2}$ & 7.3 & $3.1_{-1.0}^{+1.5}$ & 5.0 \\
115 & $2.2_{-0.8}^{+1.0}$ & 3.1 & $4.6_{-1.5}^{+2.2}$ & 6.9 & $3.3_{-1.0}^{+1.1}$ & 4.8 \\
120 & $1.8_{-0.5}^{+0.7}$ & 2.9 & $4.2_{-1.4}^{+1.9}$ & 6.7 & $2.7_{-0.7}^{+1.8}$ & 4.5 \\
125 & $1.6_{-0.4}^{+0.9}$ & 2.7 & $3.8_{-1.1}^{+1.5}$ & 6.4 & $2.5_{-0.6}^{+1.2}$ & 4.2 \\
130 & $1.6_{-0.5}^{+0.7}$ & 2.6 & $3.6_{-1.1}^{+1.5}$ & 5.9 & $2.5_{-0.8}^{+1.1}$ & 4.0 \\
135 & $1.6_{-0.4}^{+0.7}$ & 2.5 & $3.1_{-0.8}^{+1.6}$ & 5.4 & $2.2_{-0.6}^{+1.0}$ & 3.7 \\
140 & $1.5_{-0.5}^{+0.6}$ & 2.3 & $3.1_{-0.9}^{+1.3}$ & 4.9 & $2.3_{-0.8}^{+0.9}$ & 3.5 \\
\hline
\end{tabular}




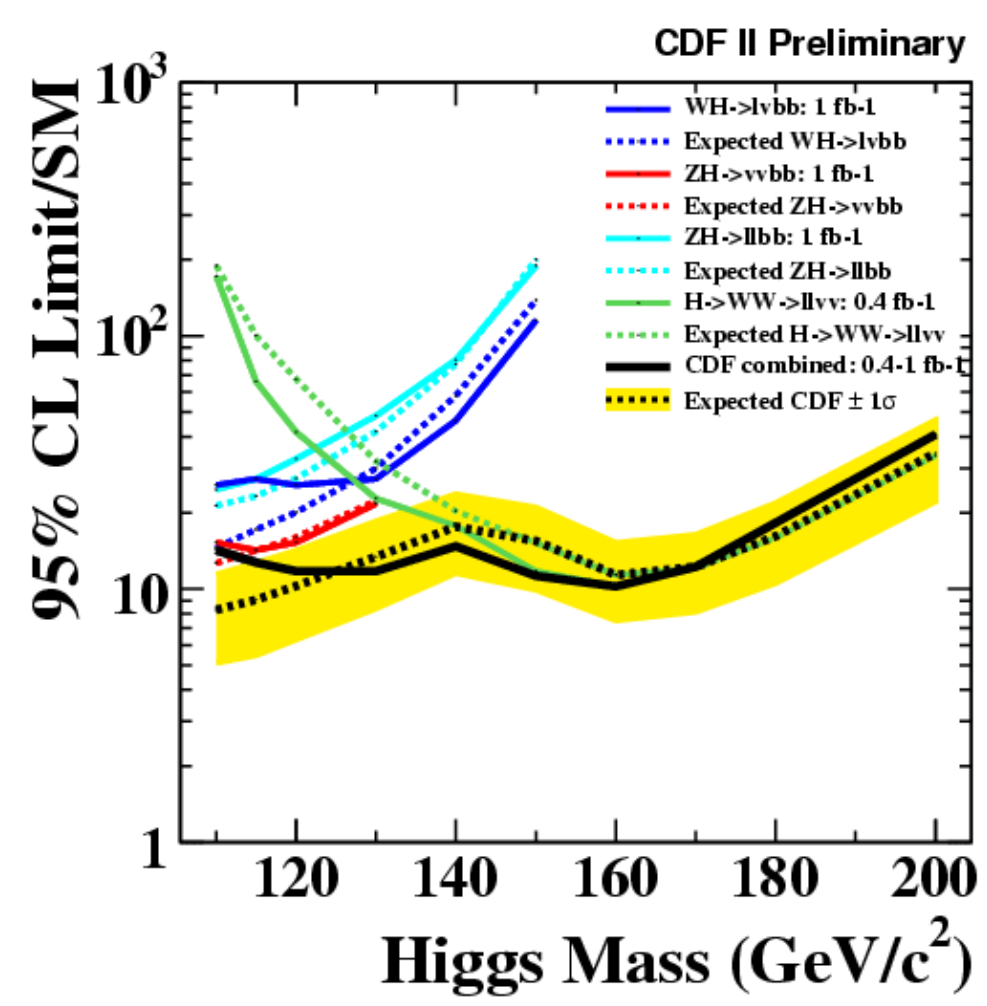

Fig. 7.2. Combined 95\% C.L. exclusion limits on the Standard Model Higgs boson cross-section at CDF

\subsection{Candidate Events}

Due to the overwhelming background in the search that can produce events very similar to our signal, we can only make statistical statements about the Higgs. Nevertheless, we have found a few candidate events that could be typical examples of a ZH signal. Similar final states are produced for example in $\mathrm{Z}+$ h.f. and diboson processes in which the $\mathrm{Z}$ decays to neutrinos and a gluon or another vector boson decays to b-quarks. Two candidate events are shown in Figure 7.4 and 7.5. In the second event, a third soft jet appears close to one of the b-jets which is a result of final state radiation, and the other jet contains a lepton which is a sign of semi-leptonic decay. 


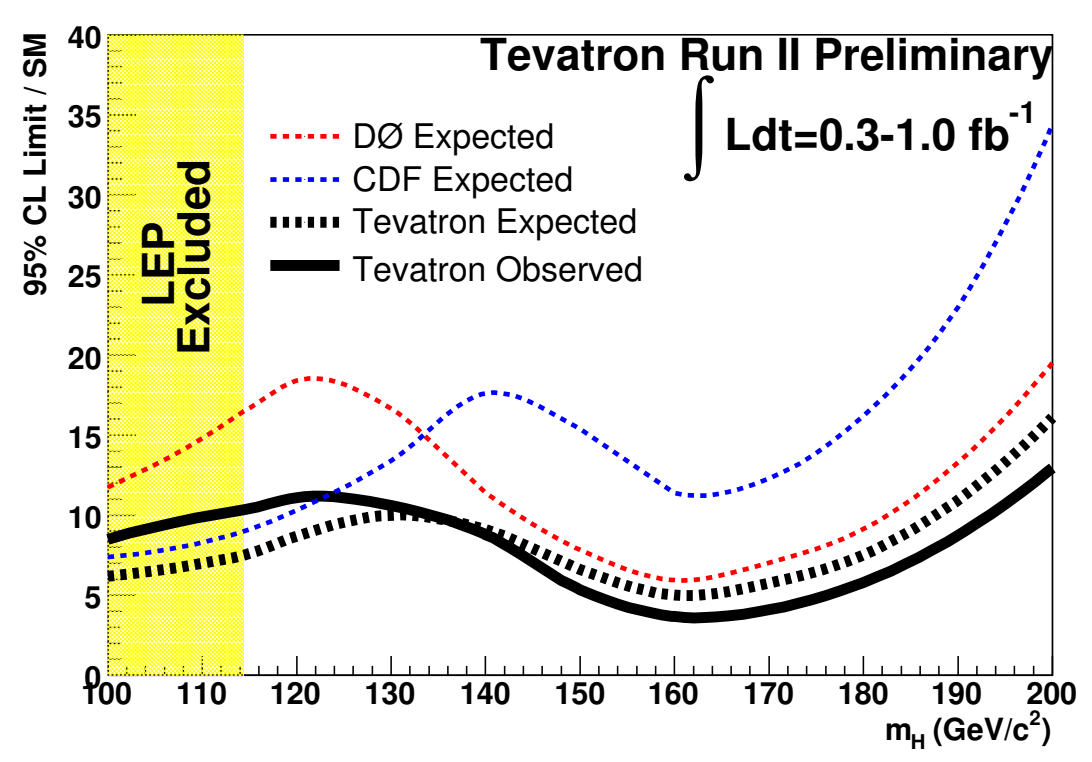

Fig. 7.3. Combined 95\% C.L. exclusion limits on the Standard Model Higgs boson cross-section at the Tevatron

\subsection{Conclusion}

We have performed the first Run II search for the Standard Model Higgs boson at CDF using $\not_{T}$ and b-jets events. The result is consistent with the Standard Model both with and without the Higgs boson. Since the current sensitivity is not sufficient to make a more specific statement, we provide an estimate for it in the form of $R_{\text {expected }}$ that is the ratio between the excluded cross-section of a Higgs-like process and the theoretical effective Higgs production cross-section.

If the SM Higgs boson existed and its mass were outside the scope of this search, an exclusion would be possible when $R_{\text {expected }} \approx 1$. The change in $R_{\text {expected }}$ is inversely proportional to the square-root of the improvement in the luminosity of the data; therefore, in order to exclude the SM Higgs with mass $115 \mathrm{GeV}$, we would need to multiply the data by a factor of $\sim 225$.

The current sensitivity is limited by the statistical error in the data. When more data is collected, the systematic uncertainties will have larger impact. Reducing 

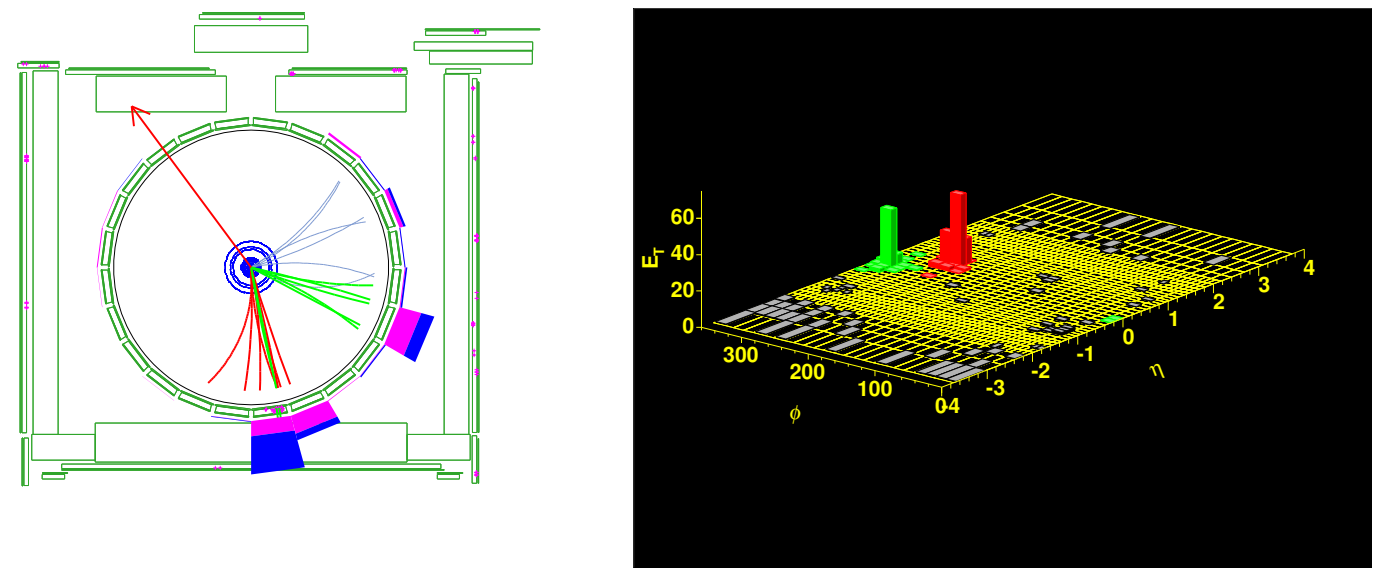

Fig. 7.4. Double tag Higgs candidate event: $E_{T}^{1}=100 \mathrm{GeV}, E_{T}^{2}=55$ $\mathrm{GeV}, \not_{T}=145 \mathrm{GeV}$, dijet mass is $82 \mathrm{GeV}$
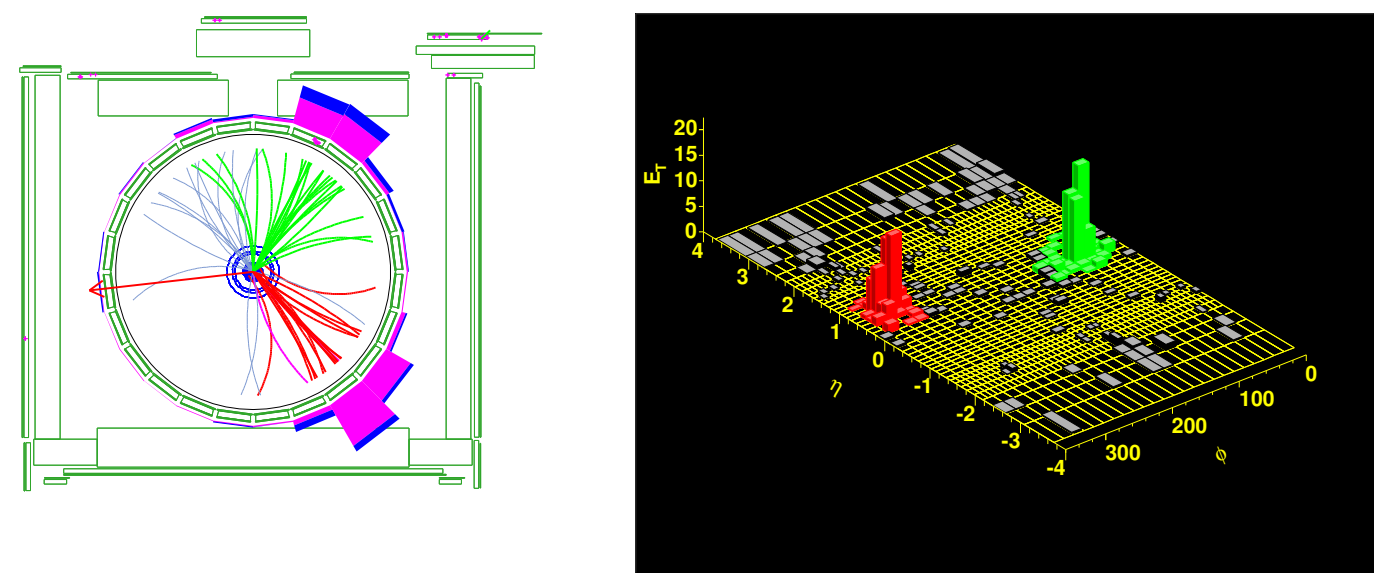

Fig. 7.5. Single tag Higgs candidate event: $E_{T}^{1}=85 \mathrm{GeV}, E_{T}^{2}=72$ $\mathrm{GeV}, \not \notin_{T}=98 \mathrm{GeV}$, dijet mass is $129 \mathrm{GeV}$ 
the systematic uncertainties will help to improve the limit. The sensitivity can be further improved by introducing more efficient b-tagging methods with higher purity for b-jets, by reducing the dijet mass resolution through b-jet specific jet-correction algorithms, and by implementing an advanced signal selection method.

The Higgs search sensitivity at CDF is the result of the combination of all searches performed in the various production channels. The latest preliminary limit combination by the CDF collaboration is $R_{\text {expected }} \approx 10$ for a light Higgs with mass below $130 \mathrm{GeV}$. The Tevatron combination is expected to be around $R_{\text {expected }} \sim 7$ at $95 \%$ C.L. 
LIST OF REFERENCES 


\section{LIST OF REFERENCES}

[1] D. H. Perkins, "Introduction to High Energy Physics" (2000), Cambridge University Press

[2] F. Halzen, A. D. Martin, "Quarks \& Leptons: An Introductory Course in Modern Particle Physics" (1984), John Wiley \& Sons, Inc

[3] L. H. Ryder, "Quantum Field Theory" $(1985,1996)$, Cambridge University Press

[4] S. Weinberg, "A Model Of Leptons," Phys. Rev. Lett. 19, 1264 (1967).

[5] A. Salam, in Elementary Particle Theory, Proc. 8th Nobel Symp., Aspenäsgården, 1968, ed. by N. Svartholm (Almqvist and Wiksell, Stockholm, 1968) p.367.

[6] P. W. Higgs, Phys. Lett. 12, 132 (1964).

[7] G. Arnison et al. [UA1 Collaboration], "Experimental Observation Of Isolated Large Transverse Energy Electrons With Associated Missing Energy At S**(1/2) = 540-Gev," Phys. Lett. B 122, 103 (1983).

[8] G. Arnison et al. [UA1 Collaboration], "Experimental Observation Of Lepton Pairs Of Invariant Mass Around 95-Gev/C**2 At The Cern Sps Collider," Phys. Lett. B 126, 398 (1983).

[9] W-M Yao et al. [Particle Data Group Collaboration], "Review of particle physics," 2006 J. Phys. G: Nucl. Part. Phys. 331

[10] M. Carena and H. E. Haber, "Higgs Boson Theory and Phenomenology", hep$\mathrm{ph} / 0208209 \mathrm{v} 3(2002)$

[11] M. Spira and P. M. Zerwas, "Electroweak Symmetry Breaking and Higgs Physics", hep-ph/9803257v2 (1998)

[12] S. Mrenna and C. P. Yuan, Phys. Lett. B416 (1998) 200.

[13] J. F. Gunion, H. E. Haber, G. Kane and S. Dawson, "The Higgs Hunter's Guide" (1990) Perseus Publishing, Cambridge, MA

[14] The LEP Electroweak Working Group http://lepewwg.web.cern.ch/LEPEWWG and

Tevatron Electroweak Working Group (TEV-EWWG) http://tevewwg.fnal.gov/

[15] G. Abbiendi et al. [the ALEPH Collaboration, the DELPHI Collaboration, the L3 Collaboration and the OPAL Collaboration, The LEP Working Group for Higgs Boson Searches] "Search for the Standard Model Higgs Boson at LEP" Phys. Lett. B565 (2003) 61-75 
[16] "Tevatron Run II Handbook", http://www-bd.fnal.gov/lug/runII_handbook/RunII_index.html.

[17] "Constraints on CDF operation in 396ns Mode", CDF/DOC/ONLINE/PUBLIC/4692.

[18] F. Abe, et al., Nucl. Instrum. Methods Phys. Res. A 271, 387 (1988); D. Amidei, et al., Nucl. Instum. Methods Phys. Res. A 350, 73 (1994); F. Abe, et al., Phys. Rev. D 52, 4784 (1995); P. Azzi, et al., Nucl. Instrum. Methods Phys. Res. A 360, 137 (1995);

[19] "The CDF-II Detector Technical Design Report", Fermilab-Pub-96/390-E.

[20] C. S. Hill for the CDF Collaboration, "Operational experience and performance of the CDFII silicon detector," Nucl. Instrum. Meth. A 530, 1 (2004).

[21] T. K. Nelson for the CDF II Collaboration, "The CDF Layer 00 Detector", Fermilab preprint, FERMILAB-CONF-01-357-E (2001).

[22] A. Affolder et al. [CDF Collaboration], "The CDF intermediate silicon layers detector," Nuovo Cim. 112A, 1351 (1999).

[23] A. Affolder et al. [CDF Collaboration], "The intermediate silicon layers detector at CDFII: Design and progress," Nucl. Instrum. Meth. A 435, 44 (1999).

[24] T. Affolder et al. [CDF Collaboration], "CDF central outer tracker," Nucl. Instrum. Meth. A 526, 249 (2004).

[25] S. Cabrera et al. [CDF Collaboration], "The CDF time of flight detector," FERMILAB-CONF-03-404-E Presented at IEEE 2003 Nuclear Science Symposium (NSS) and Medical Imaging Conference (MIC) including Sessions on Nuclear Power Systems, Portland, Oregon, 19-24 Oct 2003

[26] C. M. Ginsburg for the CDF Collaboration, "CDF Run 2 muon system," Eur. Phys. J. C 33, S1002 (2004).

[27] T. Dorigo [CDF Collaboration], "The muon system upgrade for the CDF II experiment," Nucl. Instrum. Meth. A 461, 560 (2001).

[28] J. Elias et al., "Luminosity monitor based on Cherenkov counters for p anti-p colliders," Nucl. Instrum. Meth. A 441, 366 (2000).

[29] D. Acosta et al. [CDF Collaboration], "The CDF Cherenkov luminosity monitor," Nucl. Instrum. Meth. A 461, 540 (2001).

[30] E. J. Thomson et al. Presented at IEEE 2001 Nuclear Science Symposium (NSS) and Medical Imaging Conference (MIC), San Diego, California, 4-10 Nov 2001. IEEE Trans. Nucl. Sci. 49, 1063 (2003).

[31] S. Holm et al. [CDF Collaboration], "System architecture and hardware design of the CDF XFT online track processor," IEEE Trans. Nucl. Sci. 47, 895 (2000). 
[32] "Level-2 calorimeter Trigger Upgrade at CDF", Bhatti, A. Canepa, A. Casarsa, M. Convery, M. Cortiana, G. Dell'Orso, M. Donati, S. Flanagan, G. Frisch, H. Fukun, T. Giannetti, P. Jones, M. Liu, T. Lucchesi, D. Pagan, S. Pantano, D. Piendibene, M. Ristori, L. Rogondino, L. Rusu, V. Sartori, L. Veszpremi, V. Vidal, M. Zhou, L.

Nuclear Science Symposium Conference Record, 2006. IEEE

Vol.3, 1387-1391, ISBN: 1-4244-0561-0

[33] A. Bardi et al., "SVT: An online silicon vertex tracker for the CDF upgrade", Nucl. Instrum. Meth. A 409, 658 (1998).

[34] "SVT Technical Design Report", CDF/DOC/TRIGGER/PUBLIC/3108.

[35] G. Gomez-Ceballos et al., "Event builder and Level 3 at the CDF experiment", Nucl. Instrum. Meth. A 518, 522 (2004).

[36] G. C. Blazey et al., "Run II jet physics", hep-ex/0005012

[37] A. Bhatti et al., "Determination of the Jet Energy Scale at the Collider Detector at Fermilab", Nucl. Instrum. Meth. A566 (2006) 375-412, hep-ex/0510047v1; G. Arnison et al. [UA1 Collaboration], Phys. Lett. B123, 115 (1983)

[38] D. A. Acosta et al., "Clean up of the Missing Transverse Energy Datasets", $\mathrm{CDF} / \mathrm{DOC} / \mathrm{EXOTIC} / \mathrm{CDFR} / 7410$

[39] D. Acosta et al. [CDF Collaboration], "Measurement of the t anti-t production cross section in $\mathrm{p}$ anti-p collisions at $\mathrm{s}^{* *}(1 / 2)=1.96 \mathrm{TeV}$ using lepton + jets events with secondary vertex b-tagging", hep-ex/0410041; A. Abulencia et al. [CDF Collaboration], Phys. Rev. D74, 072006 (2006); C. Neu, in Proceedings of the International Workshop on Top Quark Physics, January 12-15, 2006, Coimbra, Portugal published by POS.

[40] L. Cerrito et al., "ttbar xsec with SLT in 200pb-1" $\mathrm{CDF} / \mathrm{ANAL} / \mathrm{TOP} / \mathrm{PUBLIC} / 7174$

[41] G. Gomez et al., "Measurement of the ttbar Production Cross Section in the Jet Probability Tagged Sample", CDF/ANAL/TOP/PUBLIC/7236

[42] Public web-page of the High- $p_{T}$ B-tag group, http://wwwcdf.fnal.gov/physics/physics.html

[43] F. Garberson et al., "Combination of the SecVtx $1.2 \mathrm{fb}^{-1}$ b-Tagging Scale Factors", CDF/DOC/SEC_VTX/CDFR/8666

[44] R. G. Wagner, "Electron Identification for Run II: Algorithms", CDF Note 5456 (2003)

[45] D. Hare et al., "Electron ID Efficiency and Scale Factors for Winter 2007 Analyses", CDF/DOC/ELECTRON/CDFR/8614

[46] T. Sjostrand, P. Eden, C. Friberg, L. Lonnbald, G. Miu, S. Mrenna and E. Norrbin, Comput. Phys. Commun. 135, 238 (2001)

[47] http://root.cern.ch 
[48] CDF Collaboration, "Combination of CDF top quark pair production cross section measurements with up to $760 \mathrm{pb}^{-1}$ ", CDF/PHYS/TOP/PUBLIC/8148

[49] Z. Sullivan, Fermilab-Pub-04-142-T, hep-ph/0408049

[50] B. W. Harris et al., Phys. Rev. D 66, 054024 (2002)

[51] D. Acosta et al., [CDF Collaboration], "First Measurements of Inclusive W and Z Cross Sections from Run II of the Fermilab Tevatron Collider", Phys. Rev. Lett. 94, 091803 (2005).

[52] A. Abulencia et al. [CDF Collaboration], "Measurement of the b jet cross section in events with a $\mathrm{Z}$ boson in $\mathrm{p}[$ overline $\mathrm{p}]$ collisions at sqrt(s)=1.96 TeV", Phys. Rev. D 74, 032008 (2006).

[53] J. M. Campbell and R. K. Ellis, Monte Carlo for FeMtobarn processes, http://mcfm.fnal.gov/

[54] Carsten Rott, "Search for Scalar Bottom Quarks from Gluino Decays in Proton-Antiproton Collisions at 1.96TeV", PhD Thesis, CDF/THESIS/EXOTIC/PUBLIC/7526

[55] D. Acosta et al. [CDF Collaboration], Phys. Rev. D71 052003 (2005)

[56] S. Ginstein et al., "SecVtx Mistag Asymmetry for Winter 2007", $\mathrm{CDF} / \mathrm{DOC} / \mathrm{SEC} \_V T X / \mathrm{CDFR} / 8626$

[57] U. Glunder et al., "High-Pt muons recommended cuts and efficiencies for Winter 2007", CDF/ANAL/TOP/CDFR/8618

[58] S. Jindariani et al., "Luminosity Uncertainty for Run 2 up until August 2004", $\mathrm{CDF} / \mathrm{PUB} / 7446$

[59] CDF/PHYS/EXOTIC/CDFR/7051

[60] J. Pumplin, D. R. Stump, J. Huston, H. L. Lai, P. Nadolsky and W. K. Tung, J. High Energy Phys. 0207 (2002) 012

[61] Tom Junk, "Sensitivity, Exclusion and Discovery with Small Signals, Large Backgrounds, and Large Systematics", CDF/DOC/STATISTICS/PUBLIC/8128

[62] Joel Heinrich, "Bayesian limit software: multi-channel with correlated background efficiencies", CDF/MEMO/STATISTICS/PUBLIC/7587; J. Heinrich, C. Blocker, J. Conway, L. Demortier, L. Lyons, G. Punzi and P.K. Sinervo arXiv:physics/0409129v1 [physics.data-an].

[63] CDF Collaboration, "Combined Upper Limit on Standard Model Higgs Boson Production at CDF for Summer 2006" CDF/ANAL/EXOTIC/PUBLIC/8403

[64] The TEVNPH Working Group for the D0 and CDF Collaborations, "Combined D0 and CDF Upper Limits on Standard-Model Higgs-Boson Production", CDF/DOC/EXOTIC/CDFR/8384, D0 Note 5227

[65] J.J. Sakurai, "Modern Quantum Mechanics" (1993), Addison-Wesley 
APPENDICES 


\section{A. PARTICLES IN THE STANDARD MODEL}

According to our present knowledge of particle physics [9], everything in the Universe consists of two kinds of particles: fermions which serve as "building blocks" of matter, and bosons which are the "mortar" between these blocks. Both fermions and bosons are represented by quantized fields. The quanta of these fields are the actual particles. Fermions interact with each other by exchanging bosons. The type of interactions they can participate in are determined by their charges. Some bosons also have charges themselves and therefore are capable of interacting with each other. Each particle has a pair called anti-particle with equivalent properties, only their charges have opposite signs.

Fermions are half integer spin particles that follow the Pauli principle: in a specified quantum state there can be only one fermion present. They are described statistically by the Fermi-Dirac distribution [65].

Bosons are integer spin particles that follow the Bose-Einstein statistics [65]. They do not need to obey the Pauli principle; there is no limit to the number of bosons in a certain quantum state.

Particle physics identifies twelve fermions as elementary. There are six quarks (up, down, charm, strange, top, bottom) arranged in three families, and six leptons (electron, muon, tau, and three neutrinos) also representing three families. There is an electrically charged and a neutral lepton in each family. (Table A.1)

All the other particles are composite; they are triplets or doublets of quarks. Mesons are bound states of quark and anti-quark pairs. They have integral spins, therefore they are bosons. The interactions between the nucleons in the nucleus of an atom are not fundamental, they resemble to the Van der Waals interactions and are "mediated" by these mesons. The other group of composite particles, the baryons, 
Table A.1

Fermions in the Standard Model

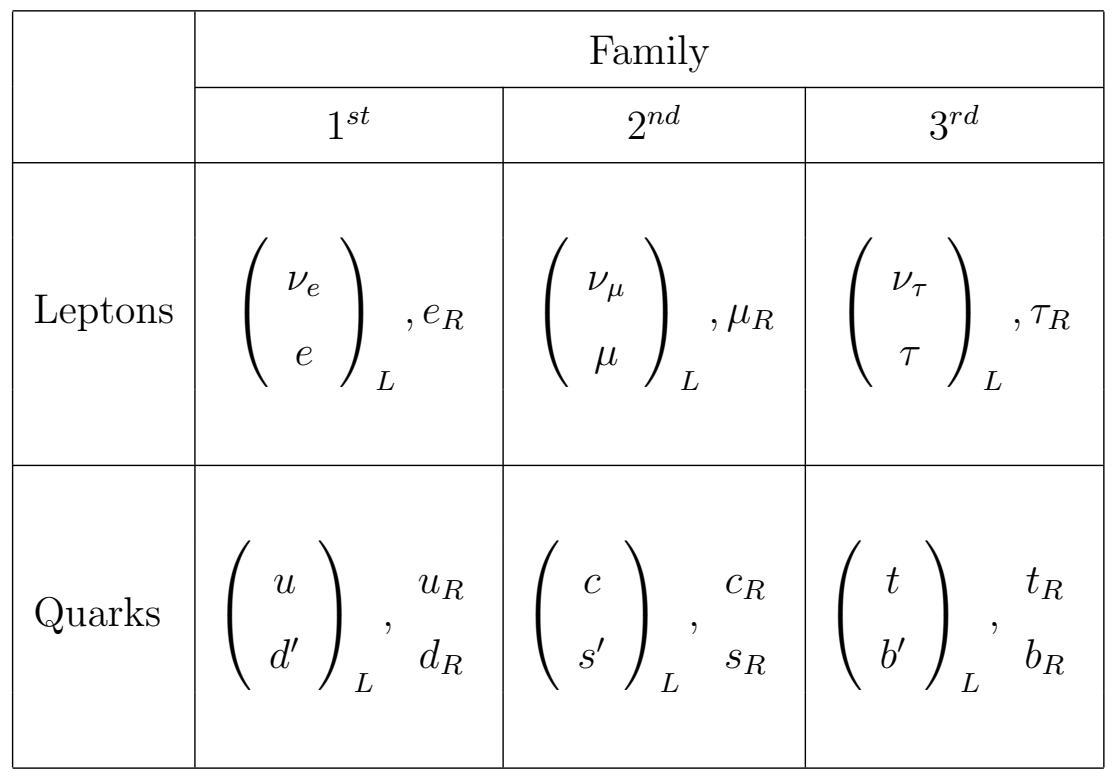

are comprised of three quarks. Protons and neutrons are the most typical example of baryons.

Particles are characterized by conserved quantities such as spin, mass, electric charge, isospin, and flavor. All the quarks and leptons are listed in Table 1.1, the bosons in Table 1.2. Composite particles have other conserved quantum numbers according to their types. We can assign positive baryon numbers $(B)$ to baryons and negative ones to anti-baryons. The baryon number of the proton is 1 by definition, thus the quarks carry one third of baryon charge. Leptons have associated lepton numbers $(L)$ which are also conserved. The only exception from this rule is observed in neutrino oscillations, when a neutrino from one family can oscillate into another family's neutrino type. A typical example of lepton number conservation is the muon decay $\left(\mu^{-} \rightarrow e^{-} \bar{\nu}_{e} \nu_{\mu}\right)$ in which the muon decays to an electron, but to preserve $L$, a muon-neutrino and an electron anti-neutrino are also produced. 


\section{B. MISSING $E_{T}$ AND BACK-UP TRIGGERS}

\section{B.1 Missing $E_{T}$ Triggers}

\section{MET35_\&_CJET_\&_JET_DPS trigger:}

- L1: $\notin_{T}>25 \mathrm{GeV}$;

- L2: 1 cluster with $E_{T}=10 \mathrm{GeV},|\eta|<3.6$;

1 cluster with $E_{T}=10 \mathrm{GeV},|\eta|<1.1$;

Dynamically changing Pre-Scale factor between 1 and 40;

- L3: \& $_{T}<35 \mathrm{GeV}$;

DPS: In order to minimize dead-time in the entire data acquisition system (for all the triggers), events collected by too active triggers are randomly discarded. The prescale factor shows how many events have to be produced on average in order for one event to be sent to the next trigger level. In DPS this factor decreases proportionally with the instantaneous luminosity.

\section{MET_BJET_LUMI_150 trigger:}

- L1: 1 cluster with $E_{T}>5 \mathrm{GeV}$; $\notin_{T}<15 \mathrm{GeV}$

$2 \mathrm{XFT}$ tracks with $p_{T}>2 \mathrm{GeV}$;

Luminosity enabled switch with range 0 to $150 \times 10^{30} \mathrm{~cm}^{-2} \mathrm{~s}^{-1}$;

- L2: 2 displaced SVT tracks with $p_{T}>2 \mathrm{GEV}, d_{0}>100 \mu m$ and $d_{0}<1000 \mu m$, and $\Delta \phi(\operatorname{track} 1, \operatorname{track} 2)>2^{\circ}$;

1 cluster with $E_{T}>15 \mathrm{GeV}$ in matching location with a displaced track;

- L3: H $_{T}>20 \mathrm{GeV}$;

2 displaced COT tracks with $p_{T}>2 \mathrm{GeV}$; 
LUMI: The trigger is switched off when the instantaneous luminosity is outside the allowed range.

\section{MET45 trigger:}

- L1: $\notin_{T}>25 \mathrm{GeV}$;

- L2: $\notin_{T}>35 \mathrm{GeV}$ using $\notin_{T}$ calculated at Level 1;

- L3: \& $_{T}>45 \mathrm{GeV}$;

MET25 back-up trigger:

- L1: $\notin_{T}>25 \mathrm{GeV}$;

- L2: Pre-scale factor PS=400;

- L3: NO REQUIREMENT;

PS is similar to DPS, but the scale factor is fixed.

\section{B.2 Triggers Used in the Trigger Study}

\section{MUON_CMUP18 trigger:}

- L1: 1 muon candidate with $p_{T}>6 \mathrm{GeV}$ and a matched XFT track with $p_{T}>4 \mathrm{GeV}$;

- L2: 1 muon candidate track (4 XFT layers) with $p_{T}>15 \mathrm{GeV}$;

- L3: 1 muon candidate with CMU and CMP hits and $P_{T}>18 \mathrm{GeV}$; 


\section{JET20 trigger:}

- L1: 1 cluster (central or plug) with $E_{T}>5 \mathrm{GeV}$;

Pre-scale factor 50;

- L2: 1 cluster with $E_{T}>15 \mathrm{GeV}$ and $|\eta|<3.6$;

Pre-scale factor 25;

- L3: 1 jet (JETCLU cone=0.7) with $E_{T}>20 \mathrm{GeV}$;

\section{JET50 trigger:}

- L1: 1 cluster (central or plug) with $E_{T}>5 \mathrm{GeV}$;

Pre-scale factor 50;

- L2: 1 cluster with $E_{T}>40 \mathrm{GeV}$ and $|\eta|<3.6$;

Pre-scale factor 2;

- L3: 1 jet (JETCLU cone=0.7) with $E_{T}>50 \mathrm{GeV}$; 
VITA 


\section{VITA}

Viktor Veszpremi was born on October 5, 1976 in Oroszlany, Hungary. He completed his high school education at the Eotvos Jozsef Gimnazium, Tata in 1995. He attended the University of Debrecen (earlier Kossuth Lajos University) between Fall 1995 and Summer 2000 where he earned his Diploma in Physics and Teacher of Physics majors. Subsequently, he entered the graduate school at the Florida Institute of Technology (FIT) and worked as a Teaching Assistant until 2003 while conducting research in Heavy Ion Physics as a member of the PHENIX Collaboration in Brookhaven National Laboratory. He received the John E. Miller Award of FIT for excellence in graduate teaching and outstanding academic excellence in 2003. In the Spring of 2003, he transfered to the Ph.D program at Purdue University. He joined the CDF Collaboration at Fermilab as a student of Daniela Bortoletto where he conducted his thesis research until receiving his Ph.D in Physics in August 2007. 